\title{
SEARCH FOR NEW PHYSICS IN FINAL STATES WITH LOW \\ TRANSVERSE ENERGY PHOTON AND MISSING TRANSVERSE ENERGY IN PROTON-PROTON COLLISIONS AT $\sqrt{s}=8$ TEV
}

\author{
by \\ Zeynep Demiragli \\ B.A. in Physics, Bogazici University, 2010 \\ A DISSERTATION SUBMITTED IN PARTIAL FULFILLMENT OF THE REQUIREMENTS FOR \\ The Degree of Doctor of Philosophy \\ in the Department of Physics at Brown University
}

Providence, Rhode Island

May 2015 
(C) Copyright 2015 by Zeynep Demiragli 
This dissertation by Zeynep Demiragli is accepted in its present form by the Department of Physics as satisfying the dissertation requirement for the degree of Doctor of Philosophy

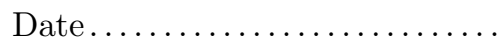

Greg Landsberg, Advisor

Recommended to the Graduate Council

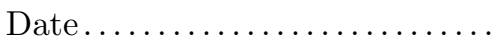

David Cutts, Reader

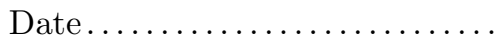

Anastasia Volovich, Reader

Approved by the Graduate Council

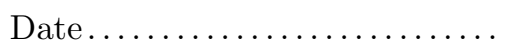

Peter M. Weber

Dean of the Graduate School 


\section{Curriculum Vitae}

\section{Zeynep Demiragli}

\section{Contact Information}

Brown University Physics Department

182 Hope Street, Providence, RI 02906
Brown Email: zeynep_demiragli@brown.edu

CERN Email: zeynep.demiragli@cern.ch

\section{Education}

$\underline{\mathbf{0 1} / 2015}$ Ph.D. Experimental High Energy Physics, Brown University

Thesis: "Search for New Physics Using the $\gamma+\not{ }_{T}$ Signature with the CMS Experiment"

Supervisor: Prof. Greg Landsberg

05/2010 B.S. Physics, Bogazici University

Senior Project: "Majorana Neutrino: Studies on Mass Term Transformations"

Supervisor: Prof. Metin Arik

\section{Research Activities}

Search for new physics in low-energy $\gamma+\mathbb{E}_{T}$ final state Designed, implemented, and commissioned the low-energy photon trigger which operated as part of the CMS "data parking" program. Led the full analysis of the $7.3 \mathrm{fb}^{-1}$ collected by this trigger at $\sqrt{s}=8 \mathrm{TeV}$, which included event selection, data driven and Monte Carlo based background estimations, sensitivity optimization, uncertainty estimation and statistical interpretation of the low photon $p_{T}$ and low missing transverse energy range. This is the first search at the LHC using the low-energy range of the $\gamma+\not_{T}$ final state. 
Search for new physics in high-energy $\gamma+\mathbb{E}_{T}$ final state Played a leading role in the analysis using full dataset collected at $\sqrt{s}=8 \mathrm{TeV}$ by independently performing several aspects of the analysis. The efforts included contributions to the event selection, sensitivity optimization, and uncertainty estimation. Most importantly, developed a new algorithm (MHT minimization) to reduce the contamination of events with mismeasured $\not_{T}$. This algorithm led to $95 \%$ reduction of the dominant instrumental Standard Model background with $>99 \%$ signal efficiency.

Search for new physics in $\gamma \gamma$ final state Computed cross section limits using an alternative statistical technique. This required parametrization of the background and signal shapes and their uncertainty estimations, which allowed the limits to be computed for a larger number of signal mass hypotheses and the possibility to combine the results with resonance searches in different final states.

Search for black holes Took part in devising the object selection, search window optimization, and estimation of various systematic uncertainties such as the uncertainty due to the choice of parton distribution functions.

\section{Service Work Activities}

Exotica Trigger Contact [2013-2014] Served as Exotica group trigger contact for the multijets, non-hadronic and lepton+jets working groups. Led the coordination of the trigger strategy for these groups for LHC Run 2. Designed and implemented the high energy single and double photon triggers in preparation for LHC Run 2.

TSG Steam Subgroup [2012-2014] Developed software (new openHLT), which is widely used by trigger developers for optimization studies. Constructed the "half rate" menu which was used as the baseline for all Run 2 HLT algorithms within CMS. Contributed to large scale trigger rate studies such as pileup dependence both for the $7 \mathrm{TeV}$ and $8 \mathrm{TeV}$ data taking conditions.

HLT On Call Expert [2012-2014] Served as a 24/7 on-call expert for the CMS HLT. Tasks included monitoring the data quality of the CMS detector, as well as designing special data 
taking configurations.

\section{Selected Publications And Conference Notes}

1) "Search for microscopic black holes in $p p$ collisions at $\sqrt{s}=8 \mathrm{TeV}$ ", JHEP 07 (2013) 10.1007, arXiv: 1303.5338

2) "Search for new physics in the monophoton final state in pp collisions at $\sqrt{s}=8 \mathrm{TeV}$ ", CMSPAS-EXO-12-047, To be submitted to Physics Letters B

3) "Search for Dark Matter and Large Extra Dimensions in the photon + met final state", Proceedings of the Second Annual Large Hadron Collider Physics Conference (LHCP), arXiv: 1409.4089

4) "Performance of e $/ \gamma$-based triggers at the CMS High Level Trigger", Proceedings of the Second Annual Large Hadron Collider Physics Conference (LHCP), arXiv:1409.4077

5) "Search for new physics in the low $E_{T}$ photon final state in pp collisions at $\sqrt{s}=8 \mathrm{TeV}$ ", In preparation

6) "Search for new physics in the diphoton final state in pp collisions at $\sqrt{s}=8 \mathrm{TeV}$ ", In preparation

\section{Talks and Posters}

07/2014 "Searches for no-SUSY Exotics (CMS)", Parallel Talk, SUSY 2014, Manchester, United Kingdom

05/2014 "Shining Light on Dark Matter", Large Hadron Collider Physics (LHCP) Conference Poster Session, New York City, U.S.A. (won a competition award)

05/2014 "Performance of e/ $\gamma$-based triggers at the CMS High Level Trigger", Large Hadron Collider Physics (LHCP) Conference Poster Session, New York City, U.S.A.

03/2013 "High Level Trigger development with OpenHLT", LHCC Student Poster Session, CERN, Geneva, Switzerland

\section{Awards}

05/2014 Large Hadron Collider Physics Conference Poster Session Award: "Shining Light on Dark Matter" 
12/2013 CMS Students and Post-Docs Achievement Award: "Development of the new OpenHLT tool for menu studies, construction of the half rate benchmark menu and continuous contribution to the trigger menu studies done in the context of the STEAM group of the TSG"

\section{Teaching}

$\underline{\text { Spring } 2013}$ Top Trigger Hands on Tutorial "Rate and Timing Estimations for Triggers"

Spring 2012 Physics 0111 "Are There Extra Dimensions Under Your Bed?" Grader

Fall/Spring 2010-11 Physics 0470, "Electricity and Magnetism" Teaching the discussion/lab sections

Fall 2011 Physics 050, "Foundations of Mechanics" Preparation of the workshop questions for the discussion sessions

\section{Research Statement}

The experiments at the Large Hadron Collider (LHC) at CERN are at the energy frontier of particle physics, searching for answers to fundamental questions of nature. My research interests are focused on exploring this magnificent physics. During the course of my dissertation, my research goal has been to test predictions of theories that look for solutions to questions that can not be answered by the standard model (SM) of particle physics alone. Due to the diversity of predictions, I have engaged in several lines of research including a search for extra spatial dimensions, a search for dark matter production, and a search for Supersymmetry (SUSY) in exotic Higgs boson decays.

Upon joining the Compact Muon Solenoid (CMS) experiment at the LHC in 2012, I became deeply involved in the studies of the CMS trigger. I first performed centralized studies of trigger rates and lead the development of a new python-based software package (OpenHLT), which is used for trigger development and fast optimization. Furthermore, by utilizing the new OpenHLT software, I created the benchmark "half rate" HLT menu for the $13 \mathrm{TeV}$ LHC run. For these studies I was given a prestigious CMS Achievement Award at the end of 2013. I also successfully developed a low-energy photon $(\gamma)$ and missing transverse energy $\left(\mathbb{E}_{T}\right)$ trigger for the CMS "data parking" program, enabling a novel new physics search which became the basis of my dissertation. This expertise eventually led to me being appointed a trigger convener of the CMS Exotica group, for which I have been tasked with coordinating the trigger development and strategy for the multijets, 
lepton+jets, and non-hadronic physics groups for the upcoming LHC run.

Using the data collected with the low-energy $\gamma+\mathbb{E}_{T}$ trigger, I conducted a search in an experimentally challenging phase space. The low-energy $\gamma+\mathbb{E}_{T}$ regime has never before been explored at the LHC. This phase space is particularly challenging compared to other searches because of the much larger background rate as well as the importance of understanding the systematic uncertainties, rather than the statistical uncertainty, which dominates in the high-energy regime. In this search, I have utilized multiple techniques based on control samples in data to describe various backgrounds that arise from misidentified objects, such as jets and electrons. One of the major challenges in this search was controlling the contamination coming from QCD multijet and $\gamma+$ jet production while maintaining high signal efficiency. Such events can produce "non-intrinsic" $\mathbb{E}_{T}$ due to the detector's imperfect jet energy resolution. To address this, I developed a new minimization algorithm which is able to effectively distinguish events with genuine $\mathbb{E}_{T}$ from events where the $\mathbb{E}_{T}$ comes from mismeasurement. With the addition of this algorithm, the sensitivity of the analysis was improved significantly. Due to the difficulty of triggering on events in this phase space in the future (due to high bandwidth requirements), I have focused on setting model independent limits that can be interpreted in terms of a wide range of theoretical models. I have also included an interpretation in terms of non-standard decays of the Higgs boson to $\gamma+\mathbb{E}_{T}$, where I have set the first limits to date on this particular model.

In addition to my main dissertation research, I have also been involved in a search for evidence of new physics using events with a high-energy photon $(\gamma)$ and large missing transverse energy $\left(\mathbb{E}_{T}\right)$. Using this final state, we were able to test several theoretical models including Arkani-Hamed-Dimopoulos-Dvali (ADD) extra dimensions and several models of dark matter. The minimization algorithm that I developed was also extremely important in this search and led to a significant increase in the sensitivity. It should be noted that this algorithm can also be applied to other searches. I have demonstrated that it can be used to recover the degradation in muon momentum resolution for high mass resonances decaying to muon pairs. This correction could lead to an improved signal sensitivity in the $Z^{\prime} \rightarrow \mu^{+} \mu^{-}$search during the upcoming $13 \mathrm{TeV}$ LHC run.

I further expanded my research experience through being involved in the search for microscopic black holes. Abundant production of black holes at the LHC is highly motivated in scenarios with extra large $\left(1 \mathrm{TeV}^{-1} \sim 1 \mathrm{~mm}\right)$ spatial dimensions, such as the ADD scenario, which predict 
low scale $(\sim 1 \mathrm{TeV})$ quantum gravity. In this search we modeled the sum of particle transverse energy $\left(S_{T}\right)$ distribution in different particle multiplicities and then looked for a broad excess at the high $S_{T}$ region. I contributed to the analysis by measuring the efficiency of the trigger used in the analysis and by optimizing the background fits in the various signal regions. The search has been published in JHEP and resulted in the most restrictive limits on a number of models to date.

Finally, I have also been involved in the search for Randall Sundrum (RS) gravitons using the $\gamma \gamma$ final state. The diphoton signature is an excellent way to observe the RS graviton because it's branching fraction to $\gamma$ pair is twice that to a lepton pair. In addition, the superior energy resolution of photons makes the signal easier to observe than in, e.g. the dijet case. For this search, I was responsible for extracting limits using a shape-based fitting technique. I took the lead in organizing a kick-off meeting to define the 2015 analysis strategy, as this analysis is considered one of the high-priority analyses for the CMS experiment at the beginning of the $13 \mathrm{TeV}$ LHC run. I plan to lead this analysis in the forthcoming run.

In conclusion, through the course of my dissertation research I have gained significant expertise in various aspects of triggering and performing searches for new physics at the LHC. I want to keep looking for physics beyond the SM involving various theories of Dark Matter and SUSY, both through direct searches, and through precision measurements. I am also very interested in searches for BSM Higgs boson decays, as well as in measuring precisely the properties of the newly discovered Higgs particle. Finally, I have a strong desire to gain more experience with detector hardware and object reconstruction. Through my trigger studies, I have realized the advantage one can gain by understanding the low-level aspects of an experiment. My dissertation research, where I was able to take of the data parking program, is a great example of such advantage. I hope to uncover similar opportunities in my future research. 


\section{Dedication}

To my family and loved ones who have inspired me all my life. 


\section{Preface and Acknowledgments}

First and foremost I want to thank my advisor, Greg Landsberg for his continuous support of my research. I especially thank him for giving me the intellectual freedom to follow my passion in physics even as a young graduate student. I could not have imagined having a better advisor and mentor for my Ph.D study. I would also like to thank the rest of my dissertation committee: David Cutts and Anastasia Volovich, for their support and input on this thesis.

I would like to further thank all my collaborators on the CMS experiment for all that they have taught me which allowed for the completion of this thesis and those who worked together with me on this physics analysis in particular, Mani Tripathi, Andrew Askew, Toyoko Orimoto, and Sushil Chauhan. I also would like to thank Roberto Carlin, Andrea Bocci, Simone Gennai, Muriel Vander Donckt, Roberta Arcidiacono, and Sam Harper for making working on HLT a great experience. Also many thanks to all my colleagues at Brown University on the CMS experiment, in particular John Paul Chou, Alexey Ferapontov, Ted Laird, Grant Christopher, Edmund Berry, Juliette Alimena, and Alex Garabedian who were always willing to give me advice, and at times even a place to stay. My thanks further goes to all the other graduate students at Brown University who made me feel at home from day one. Without you and various card games you have taught me, I wouldn't have survived the stress of the graduate school.

I want to thank my family Levent, Deniz, and Emrah for their unconditional support through out my life. Thank you dad, for igniting the passion for science in me at an early age, you have given me the most precious gift. Thank you mom for being my creative inspiration and helping keep the artist in me while growing up. Thank you brother for sharing my childhood with me, and even more my grown-up dreams. Lastly, David, I can't thank you enough to show how much I value all your support and love. 


\section{Contents}

Curriculum Vitae $\quad$ iv

Dedication $x$

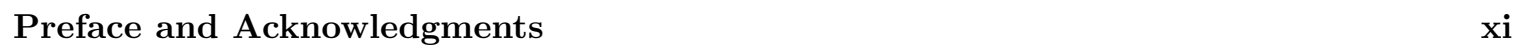

Contents $\quad$ xii

List of Tables $\quad$ xiv

List of Figures $\quad$ xvi

$\begin{array}{lll}1 & \text { Introduction } & 1\end{array}$

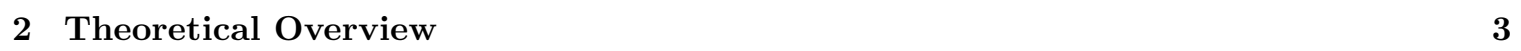

2.1 The Standard Model . . . . . . . . . . . . . . . . . . . . . . . . . . . . . . . . 4

2.1 .1 Quantum Chromodynamics . . . . . . . . . . . . . . . . . . . 4

$2.1 .2 \quad$ Electroweak Model $\ldots \ldots \ldots \ldots \ldots$

2.1 .3 Higgs Boson and Electroweak Symmetry Breaking . . . . . . . . . . . . . 8

2.2 Beyond the Standard Model . . . . . . . . . . . . . . . . . . . . . . . . . . . . . . 11

2.2 .1 Supersymmetry . . . . . . . . . . . . . . . . . . . . . . 12

2.2.1.1 A Simplified Model for the Low-Scale SUSY Breaking Scenerio . . . 13

$\begin{array}{lll}3 & \text { Experimental Apparatus } & 16\end{array}$

3.1 The Large Hadron Collider $\ldots \ldots \ldots \ldots \ldots$

3.2 The CMS Experiment $\ldots \ldots \ldots \ldots \ldots$

$3.2 .1 \quad$ CMS Tracking System $\ldots \ldots \ldots \ldots$. . . . . . . . . . . . . . . . . 21

$3.2 .2 \quad$ CMS Electromagnetic Calorimeter . . . . . . . . . . . . . . . . . . 22 
3.2 .3 CMS Hadron Calorimeter . . . . . . . . . . . . . . . . . . . . . . . . . . . . . 24

3.2 .4 CMS Muon System. . . . . . . . . . . . . . . . . . . . . . . . . . 25

3.2.5 CMS Trigger and Data Acquisition Systems . . . . . . . . . . . . . . . . . . . 26

3.2.5.1 Level 1 . . . . . . . . . . . . . . . . . . . . 27

3.2.5.2 High-Level Trigger . . . . . . . . . . . . . . . . . . . . . 28

$3.2 .5 .3 \mathrm{DAQ} \ldots \ldots \ldots \ldots \ldots \ldots \ldots$

4 Event and Object Reconstruction

4.1 Trigger Design and Implementation . . . . . . . . . . . . . . . . . . . . . . . . . . . . 31

4.2 Photon Reconstruction and Identification . . . . . . . . . . . . . . . . . . . . . . . 36

4.3 Missing Transverse Energy . . . . . . . . . . . . . . . . . . . . . . . . . . . . . . . . . 39

4.3.0.4 $\not_{T}$ Minimization. . . . . . . . . . . . . . . . 44

5 Search for new physics in final states with photon and missing transverse energy 49

5.1 Signal Modeling . . . . . . . . . . . . . . . . . . . . . . . . . . . . . . . 49

5.2 Event Selection . . . . . . . . . . . . . . . . . . . . . . . . . . 50

5.3 Background Modeling . . . . . . . . . . . . . . . . . . . . . . . . . . 53

$5.3 .1 \quad$ Simulation-Based Backgrounds . . . . . . . . . . . . . . . . . . . 54

5.3 .2 Backgrounds Determined from Data . . . . . . . . . . . . . . . . . . . . . 58

5.3.2.1 $\quad$ Estimation of electron $\rightarrow$ photon mismeasurement ratio . . . . . . . 58

$5.3 .2 .2 \quad$ Estimation of jet $\rightarrow$ photon mismeasurement ratio . . . . . . . . . 65

$5.3 .2 .3 \quad$ Non-Collision Backgrounds . . . . . . . . . . . . . . . . . . 71

5.4 Background Modeling Validation . . . . . . . . . . . . . . . . . . . . . . . . . . . . 74

5.5 Systematic Uncertainties . . . . . . . . . . . . . . . . . . . . . . . . . 76

5.6 Results . . . . . . . . . . . . . . . . . . . . . . . . . . . . . . 76

5.6 .1 Model-Independent Limits . . . . . . . . . . . . . . . . . . . 78

5.6 .2 Model-Specific Limits . . . . . . . . . . . . . . . . . . . . . 80

5.6 .3 Extension to High-Energy Regime . . . . . . . . . . . . . . . . . . . . . . 82

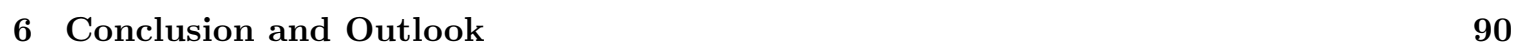

\begin{tabular}{|r|}
\hline Bibliography \\
\hline
\end{tabular} 


\section{List of Tables}

$4.1 \quad$ Triggers used for the main analysis and for the efficiency measurement. . . . . . . . 35

4.2 The fit parameters for the trigger efficiency curves $\ldots \ldots \ldots \ldots$

4.3 Fractions of photons and electrons, in the ECAL barrel and endcap, passing the pixel track seed veto. The statistical uncertainties in the values given for electrons are negligible. . . . . . . . . . . . . . . . . . . . . . . . 38

4.4 Photon identification requirements for three working points, corresponding to selections of different stringency. . . . . . . . . . . . . . . . . . . . . . . . . . . . . 40

4.5 Scale factors for the photon identification used for the analysis . . . . . . . . . . . . 40

4.6 The parameters for the $\vec{E}_{\mathrm{T}} \phi$-asymmetry corrections for x-axis projection of PF $\vec{E}_{\mathrm{T}}$ for data and simulation. . . . . . . . . . . . . . . . . . . . . . . . . 41

4.7 The parameters for the $\overrightarrow{\mathscr{E}}_{\mathrm{T}} \phi$-asymmetry corrections for y-axis projection of PF $\vec{H}_{\mathrm{T}}$ for data and simulation. . . . . . . . . . . . . . . . . . . . . . . . . 41

4.8 Scale Factors for the resolution difference in data and simulations . . . . . . . . . . . 45

4.9 Scale Factors for the $\not_{T}{ }_{T}$ resolution difference in data and MC simulation using a $\gamma+$ jet sample. . . . . . . . . . . . . . . . . . . . . . . . . 46

5.1 Summary of selection for both model independent analysis and analysis with SUSY benchmark model with the cumulative efficiencies of the selection requirements relative to the preselection for $Z \gamma \rightarrow \nu \bar{\nu} \gamma, \gamma+$ jet and $M_{\widetilde{\chi}_{1}^{0}}=120 \mathrm{GeV}$. . . . . . . . . . . 54

5.4 Event yields for data vs. SM expectation in a control region enriched by (a) $\gamma+$ jet

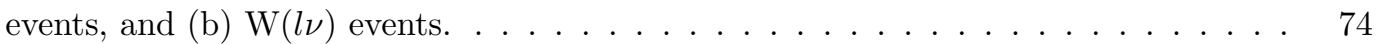

5.5 Summary of all systematic relative uncertainties in percent on $A \times \epsilon_{M C}$ calculation for SUSY Higgs model (Model Independent) selection. . . . . . . . . . . . . . . . . 78

5.6 Comparison of event yields for observed data and background, after the modelindependent selection. 
5.7 Expected (SM background) and observed event yields after the selection optimized for the supersymmetric decay of the Higgs boson $\left(h \rightarrow \widetilde{G} \widetilde{\chi}_{1}^{0}, \widetilde{\chi}_{1}^{0} \rightarrow \widetilde{G} \gamma\right) . . . . . \quad . \quad 80$

5.8 Signal predictions at $8 \mathrm{TeV}$ in gluon-fusion channel. The signal predictions correspond to $\mathrm{BR}(\mathrm{H} \rightarrow$ invisible $+\gamma)=100 \%$ assuming the SM $\mathrm{H}$ cross section at the given $m_{\mathrm{H}}$ hypothesis. Statistical and systematic uncertainties are quoted. . . . . . . . . . . 80

5.9 Summary of estimated backgrounds and observed total number of candidates. Backgrounds listed as "Others" include the small contributions from, $Z \rightarrow l l \gamma, \gamma \gamma$ and $\gamma+$ jet. Uncertainties include both statistical and systematic contributions, and the total systematic uncertainty takes into account correlations in the individual estimates. . . . . . . . . . . . . . . . . . . . . 83

5.10 Observed (Expected) 95\% CL and 90\% CL upper limits on $\sigma \times A$ as a function of the cut on the $E_{T}^{\gamma}$ for a generalized model of new physics. The requirement on $\mathbb{F}_{T}$ is fixed at $140 \mathrm{GeV} . \ldots \ldots \ldots$. . . . . . . . . . . . . . . . 84

5.11 Theoretical DM production cross section where the generated photon transverse momentum is greater than $130 \mathrm{GeV}$ and the cut-off scale $\Lambda$ is $10 \mathrm{TeV}$, observed (expected) 90\% CL upper limits on the DM production cross section $\sigma, 90 \%$ CL lower limits on the cut-off scale $\Lambda$ and the 90\% CL upper limits on the $\chi$-nucleon cross section for the vector operator as a function of the DM mass. . . . . . . . . . . . . . . . . . . . 85

5.12 Theoretical DM production cross section where the generated photon transverse momentum is greater than $130 \mathrm{GeV}$ and the cut-off scale $\Lambda$ is $10 \mathrm{TeV}$, observed (expected) 90\% CL upper limits on the DM production cross section $\sigma, 90 \%$ CL lower limits on the cut-off scale $\Lambda$ and the 90\% CL upper limits on the $\chi$-nucleon cross section for the axial vector operator as a function of the DM mass. . . . . . . . . . . . . . . . . 85

5.13 Observed and expected 95\% CL upper limits on $M_{D}$ as a function of $n$. . . . . . 88 


\section{List of Figures}

$1.1 \quad$ Feynman diagrams representing quadratically divergent loop corrections to the Higgs boson mass. . . . . . . . . . . . . . . . . . . . . . . . . . . . . . 2

2.1 The known fundamental particles of nature. . . . . . . . . . . . . . . . . . . . . . . 4

2.2 Summary of measurements of $\alpha_{s}$ as a function of the energy scale $Q[16]$. . . . . . . 6

2.3 Potential $V(\phi)$ with $\mu^{2} \geq 0$ (left) and $\mu^{2}<0$ (right). From Ref. 30$]$. . . . . . . . . . 9

2.4 Feynman diagrams for the Higgs boson production in (a) gluon fusion, (b) vectorboson fusion, (c) associated production with a gauge boson, and (d) associated production with top quarks. From Ref. $[6] \ldots$. . . . . . . . . . . . . . . . . . . . 11

2.5 The SM Higgs boson production cross sections, as a function of the center-of-mass energy, for pp collisions. The theoretical uncertainties are indicated as a band. From Ref. $[6] . \ldots \ldots \ldots \ldots \ldots \ldots \ldots \ldots \ldots$

2.6 The field content of the MSSM. . . . . . . . . . . . . . . . . . . . . . . . . . . . . 13

$3.1 \quad$ A schematic drawing of the CERN accelerator complex. From Ref. [48]. . . . . . . . 17

3.2 Integrated luminosity delivered by the LHC to the CMS experiment during the 2011 run at $\sqrt{s}=7 \mathrm{TeV}$ (left) and 2012 run at $\sqrt{s}=8 \mathrm{TeV}$ (left). From Ref. [53] . . . . 18

$3.3 \quad$ A perspective view of the CMS experiment. From Ref. [56]. . . . . . . . . . . . . . . 20

3.4 Schematic cross section through the CMS tracker in the $r-z$ plane. From Ref. [54]. 21

3.5 Total thickness of the tracker material traversed by a particle produced at the nominal interaction point, as a function of pseudorapidity, expressed in units of radiation length (left) and nuclear interaction length(right). From Ref. [59]. . . . . . . . . . . . . . . 22

3.6 A schematic design of the CMS ECAL. From Ref. 54$]$. . . . . . . . . . . . . . . . . 23

$3.7 \quad$ A schematic drawing of the CMS HCAL in the $r-z$ plane. The dashed lines denote different values of pseudorapidity. From Ref. [54]. . . . . . . . . . . . . . . . . . . . . 24 
3.8 Quarter-view of the CMS detector with CSCs of the endcap muon system highlighted in red. From Ref. $[54]$. . . . . . . . . . . . . . . . . . . . . . . . . . 26

3.9 Overview of the CMS L1 trigger. . . . . . . . . . . . . . . . . . . . . . . . . . . . . . 27

3.10 Cross section of the HLT menu in 2012 as a function of the instantaneous luminosity. From Ref. 68]. . . . . . . . . . . . . . . . . . . . . . . . . . 29

3.11 Simplified sketch of the CMS DAQ system. From Ref. 69]. . . . . . . . . . . . . . . 30

$4.1 \quad$ Pictorial representation of the transverse energy calculation of an $\mathrm{e} / \gamma$ deposition. . . 32

$4.2 \mathrm{~L} 1 \mathrm{e} / \gamma p_{\mathrm{T}}$ resolution for electrons located in the Barrel (left) and in the Endcaps (right). For both distributions, a Crystal-Ball fit is superimposed. In the right pane, the red solid line shows the result after applying the transparency corrections (see text). 33

4.3 Electron trigger efficiency at L1 ("EG" threshold : $20 \mathrm{GeV} E_{\mathrm{T}}$ ), as a function of $E_{\mathrm{T}}$

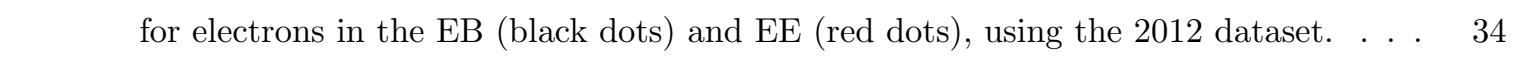

4.4 The left plot shows how the rate is gradually reduced by the filtering steps of this

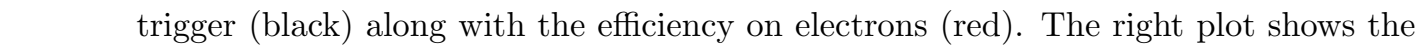
electron efficiency as a function of offline $E_{T}$ in the EB and EE before and after the transparency corrections at HLT . . . . . . . . . . . . . . . . . . . . . . . . 34

4.5 Efficiency of the HLT photon selection as a function of the offline reconstructed photon $p_{\mathrm{T}}$ (left) and efficiency of the HLT $\not_{T}$ selection as a function of the offline reconstructed particle-flow $\not_{T}$. . . . . . . . . . . . . . . . . . . . . . . 36

4.6 Fractional photon energy resolution measured in bins of absolute supercluster pseudorapidity in $Z \rightarrow \mathrm{e}^{+} \mathrm{e}^{-}$events, for data (black points) and simulated events (red points), where the electrons are reconstructed as photons. From Ref. $[73]$. . . . . . 37

4.7 Distributions in the $R_{9}$ variable for photons in the ECAL barrel that convert in the material of the tracker (solid filled histogram), and those that convert later, or do not convert at all before reaching the ECAL (outlined histogram). From Ref. [73] . . . . 38

4.8 Mean value of the isolation variables for photons with $p_{\mathrm{T}}>50 \mathrm{GeV}$ in $\gamma+$ jet events, \begin{tabular}{|c|}
\hline as a function of the number of reconstructed primary vertices, for events (left) before \\
\hline
\end{tabular}

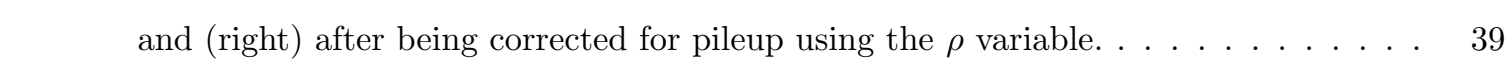

4.9 MET $\phi$ modulation correction fits for MC simulation (top row), and data (bottom

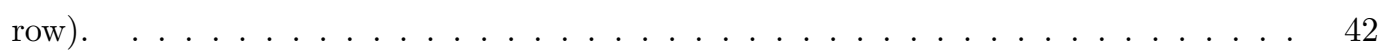

$4.10 \phi$ distributions before (a) and after (b) the correction in data and simulation. . . . . 43 
4.11 The jet transverse momentum resolution as a function of transverse momentum in 12 different $\eta$ ranges (see also Fig. 4.12 . . . . . . . . . . . . . . . . . . . . . . . . . . . 46

4.12 The jet transverse momentum resolution as a function of transverse momentum in 12 different $\eta$ ranges (continued, see also Fig. 4.11] . . . . . . . . . . . . . . . . . . . . . 47

4.13 The $E_{x, y}$ resolution as a function of $\sum E_{T}$. . . . . . . . . . . . . . . . . . . . . . 47

4.14 The probability value of the $\chi^{2}$ function and the recalculated minimized $\mathbb{F}_{T}$ distributions in data and simulation. . . . . . . . . . . . . . . . . . . . . . . . . . . . . . . 48

5.1 Feynman diagrams of a Higgs boson decay to gravitino LSP and a neutralino NLSP, which subsequently decays to a gravitino and photon. . . . . . . . . . . . . . . . . 50

5.2 The shape difference between signal $Z \nu \nu \gamma$ (blue) and background $\gamma+$ jets (red) like processes, along with the performance curves. . . . . . . . . . . . . . . . . . 53

5.3 The optimization plots on photon energy working point, MT and Met Significance. . 54

5.4 Number of Jets above $30 \mathrm{GeV}$ for PYTHIA (left) and MADGRAPH (right) simulation - samples . . . . . . . . . . . . . . . . . . . . . . . 5 56

5.5 The $E_{\mathrm{T}}^{\gamma}$ (left) and $\mathrm{N}($ jets) (right) distributions in the $\gamma+$ jet control region. . . . . . 57

5.6 (a) Unity-normalized distributions of the number of reconstructed vertices in data and

$\gamma+$ jet MC before and after the pileup reweighting. (b) Distribution of the number of vertices after the analysis selection for observed data and predicted background. . . 58

5.7 Fit of the $Z$ invariant mass for the $Z \rightarrow e \gamma$ case and the $Z \rightarrow e \gamma_{e}$ case. . . . . . . . . 61

5.8 Misidentification rate vs. probe $p_{T}$, number of primary vertices and track multiplicity. 62

5.9 Control sample distribution of photon $p_{T}, \eta$, and $\phi . \quad$. . . . . . . . . . . . . . . . . . 63

5.10 PV matched closure test in probe $p_{T}$, number of reconstructeed primary vertices and track multiplicity . . . . . . . . . . . . . . . . . . . . . . . . 64

5.11 Raw misidentification ratio values per photon $E_{\mathrm{T}}$ bin. . . . . . . . . . . . . . . . . . 66

$5.12 \sigma_{i \eta i \eta}$ templates for different photon $E_{\mathrm{T}}$ bin. . . . . . . . . . . . . . . . . . . . . . . 67

$5.13 \sigma_{i \eta i \eta}$ templates for different photon $E_{\mathrm{T}}$ bin. . . . . . . . . . . . . . . . . . . . . . 68

5.14 Final corrected misidentification ratio as a function of photon $E_{\mathrm{T}}$. . . . . . . . . . . 69

5.15 Corrected misidentification ratio as a function of photon $E_{\mathrm{T}}$ for different denominator definitions and the final corresponding yields in the analysis data. . . . . . . . . . . . 69

5.16 Corrected misidentification ratio as a function of photon $E_{\mathrm{T}}$ for different template binning. ........................... . . 70

5.17 Corrected misidentification ratio as a function of photon $E_{\mathrm{T}}$ for different $\not_{T}$ regions. $\quad 70$ 
5.18 Corrected misidentification ratio as a function of photon $E_{\mathrm{T}}$ for different sideband

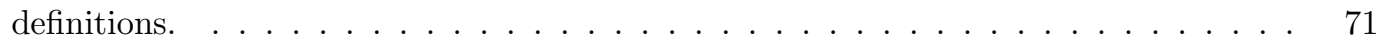

5.19 Closure test on the method. Misidentification ratio method and MC truth misidenti-

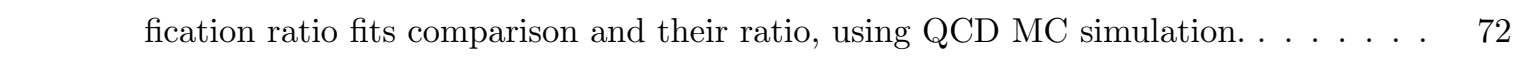

5.20 Seed time distribution (ns) for candidate events and prompt events. . . . . . . . . . 72

(a) candidate sample . . . . . . . . . . . . . . . . . . . . . . . . 72

(b) with pixel seed match . . . . . . . . . . . . . . . . . . 72

5.21 Seed time distribution $(\mathrm{ns})$ for spike and halo events . . . . . . . . . . . . . . . . 73

(a) with unphysically small shower shape variables . . . . . . . . . . . . . . 73

(b) MIP Tagged events. . . . . . . . . . . . . . . . . . . . . . 73

5.22 Template fits to the candidate selection and model independent analysis selection. . 74

5.23 The $E_{\mathrm{T}}^{\gamma}$ distributions for data vs. SM expectation in a control region enriched by (a) $\mathrm{W}(l \nu)$ events, and (b) $\gamma+$ jet events. The bottom panels in each plot show the ratio of (data - background)/background and the gray band includes both the statistical and systematic uncertainty in the background prediction. . . . . . . . . . . . . . . 75

5.24 Data vs background prediction comparison for the $\Delta \Phi\left(\gamma, \not_{T}\right)$ before and after the

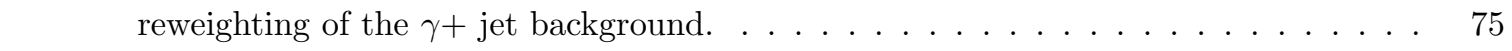

5.25 Data vs background prediction comparison for the MT distribution before and after

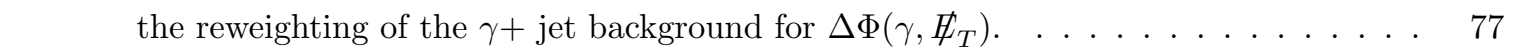

5.26 Number of Jet (left) and HT (right) distributions in the $\gamma+$ Jet control Region. . . 77

5.27 The $M_{T}$ and $\not_{T}$ distributions for data, background estimates, and signal after the model-independent selection. The bottom panels in each plot show the ratio of (data - background)/background and the gray band includes both the statistical and systematic uncertainty on the background prediction. . . . . . . . . . . . . . . . . . . . 79

5.28 The expected (a) and observed (b) 95\% CL upper limit on $\sigma \times B R \times A \times \epsilon$ for different $M_{T}$ and $\not_{T}$ thresholds and (c) for $M_{T}>100 \mathrm{GeV}$ as function of the $\not_{T}$ threshold. .79

5.29 Expected and observed 95\% CL upper limits on (a) $\sigma \times B$ and (b) the ratio of this product over the SM Higgs production cross section as a function of different $M_{\widetilde{\chi}_{1}^{0}}$ values. The uncertainty on the expected limit at $1 \sigma$ and $2 \sigma$ levels are shown as green and yellow bands, respectively. . . . . . . . . . . . . . . . . . . . . . . . . . . . . 81 
5.30 Expected and observed 95\% CL upper limits on mass with $\sigma \times \mathcal{B}$ and $\sigma \times \mathcal{B} / \sigma_{S M}$ as a function of $\tau_{\chi_{1}^{0}}$ for $m_{\mathrm{H}}=125 \mathrm{GeV}$ and $m_{\chi_{1}^{0}}=95 \mathrm{GeV}$ (up), where $\mathcal{B} \equiv \mathcal{B}(\mathrm{H} \rightarrow$ $\left.\chi_{1}{ }^{0} G\right) \times \mathcal{B}\left(\chi_{1}{ }^{0} \rightarrow G+\gamma\right)$ and on $\sigma \times \mathcal{B}$ as a function of the Higgs boson mass with $m_{\chi_{1}}^{0}=m_{\mathrm{H}}-30 \mathrm{GeV}$ (bottom) $\ldots \ldots \ldots \ldots \ldots \ldots$. . . . . . . . 81

5.31 The photon $E_{T}$ and $\not_{T}$ distribution for the candidate sample, compared with estimated contributions from SM backgrounds and the prediction from ADD for $M_{D}=2$ $\mathrm{TeV}$ and $n=3$. Background uncertainty includes statistical and systematic uncertainties . . . . . . . . . . . . . . . . . . . . 83

$5.3295 \%$ upper limits on $\sigma \times A$ as a function of the cut on the photon $E_{T}$. . . . . . . 84

5.33 The 90\% CL upper limits on the $\chi$-nucleon cross section as a function of $M_{\chi}$ for spin independent (left) and spin dependent (right) scattering. Also shown are the limits from selected experiments with published $[113[121]$ results. . . . . . . . . . . 86

5.34 Observed limits on the DM mediator mass divided by coupling, $M / \sqrt{g_{\chi} g_{q}}$, as a function of the mediator mass $M$, assuming vector interactions, for DM particle masses of $50 \mathrm{GeV}$ and $500 \mathrm{GeV}$. The width, $\Gamma$, of the mediator is varied between $M / 8 \pi$ and $M / 3$. The dashed lines show contours of constant coupling. . . . . . . . . . . . . 87

5.35 Observed limits on the DM mediator mass divided by coupling, $M / \sqrt{\overline{g_{\chi} g_{q}}}$, as a function of the mediator mass $M$, assuming vector interactions, for DM particle masses of $50 \mathrm{GeV}$ and $500 \mathrm{GeV}$ for the low energy search. The width, $\Gamma$, of the mediator is varied between $M / 8 \pi$ and $M / 3$. The dashed lines show contours of constant coupling. 87

5.36 Limits on $M_{D}$ as a function of $n$, compared to results from similar searches at the Tevatron $[125] 126$ and LEP $[127] \ldots \ldots \ldots \ldots \ldots$. . . . . . . . . 88

5.37 (a) The 95\% CL upper limits on the branon cross sections as a function of $M_{B}$ for $f=100,200,300$ and $400 \mathrm{GeV}$. (b) Limits on $f$ as a function of $M_{B}$, compared to results from similar searches at the LEP $[131] . \ldots \ldots \ldots$. . . . . . . . . 89 


\title{
Chapter 1
}

\section{Introduction}

\author{
"Begin at the beginning," the King said gravely, "and go \\ on till you come to the end: then stop." \\ - Lewis Carroll, Alice in Wonderland
}

The observation of a new boson by the CMS [1] and the ATLAS 2] collaborations with a mass of approximately $125 \mathrm{GeV}$ was an important milestone in the decades long hunt for understanding of the mechanism of electroweak symmetry breaking. In light of this observation, the 2013 Nobel Prize in Physics was awarded to Francois Englert and Peter Higgs for the development of spontaneously broken local gauge theories and theoretical insights on the origin of mass 3$]$ [4] [5]

The properties of the Higgs boson have been extensively studied by the ATLAS 6] and the CMS 7] collaborations. The mass of the Higgs particle has been measured to be $m_{H}=125.5 \pm$ 0.2 (stat) ${ }_{-0.6}^{+0.5}$ (sys) $\mathrm{GeV} / \mathrm{c}^{2}$ by ATLAS with a combined signal strength defined as $\sigma / \sigma_{S M}$ of $\mu=$ $1.30 \pm 0.12(\text { stat })_{-0.11}^{+0.14}$ (sys) and $m_{H}=125.03 \pm 0.27$ (stat) \pm 0.15 (sys) $\mathrm{GeV} / \mathrm{c}^{2}$ and by CMS with a combined signal strength of $\mu=1.00 \pm 0.09$ (stat) \pm 0.07 (sys).

The observed mass of the new particle falls in a range where the Standard Model (SM) could remain a consistent theory up to the Plank scale. However, presence of a Higgs particle with an EW-scale mass appears unnatural if the only physics present up to some high scale $\Lambda$ is the SM. If there were no new physics, then the Higgs boson mass would have received large corrections $\mathrm{O}(\Lambda)$. Consider the 1-loop Feynman diagrams in Fig. 1.1.

These diagrams give contributions to the mass of the Higgs boson, which are quadratic in the 

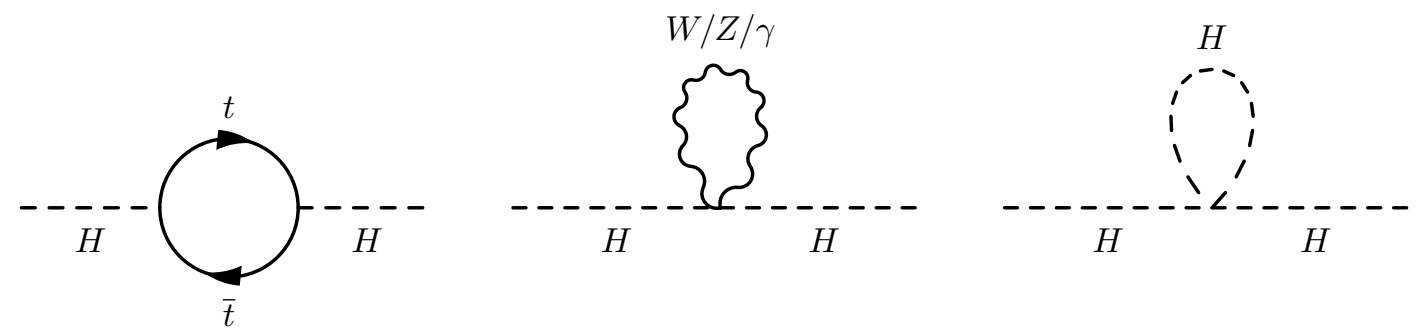

Figure 1.1: Feynman diagrams representing quadratically divergent loop corrections to the Higgs boson mass.

cutoff scale, i.e. $\Delta M^{2} \sim \Lambda^{2}$. Therefore if the cutoff scale $\Lambda$ is of the order of the Planck scale of $10^{19} \mathrm{GeV}$, then the SM parameters must be fine tuned to approximately one part in $10^{19}$ to recover the observed Higgs boson mass. In addition to this naturalness problem, the SM does not explain the existence of the so-called "dark matter" in the universe, which is roughly 5 times more abundant than the ordinary matter.

Many extensions of the SM have been proposed attempting to address these shortcomings, and the Large Hadron Collider (LHC) at CERN, together with the gigantic particle detectors known as the CMS and ATLAS experiments, is one of the few places on earth where the predictions of these theories can be tested. This thesis explores the $\gamma+\not \phi_{T}$ experimental signature predicted in several extensions to the SM using data collected by the CMS experiment. In Chapter 2, the theoretical framework of the SM will be described, as well as an overview of extensions to the SM which can be tested using the $\gamma+\not{ }_{T}$ signature. Chapter 3 will describe the experimental setup of the LHC and the CMS experiment and the reconstruction of collision events will be described in Chapter 4. The analysis of the $\gamma+\not \phi_{T}$ signature used to place constraints on new physics scenarios will be presented in Chapter 5. Finally, the conclusions and an outlook for future studies will be discussed in Chapter 6. 


\section{Chapter 2}

\section{Theoretical Overview}

Atoms were first postulated in the 5th century B.C. by the Greek philosopher Democritus, and until the beginning of the 19th century, they were thought to be indivisible building blocks of all forms of matter. The modern era of particle physics began at the end of the 19th century with the discovery of the first subatomic particle, the electron, in 1897 by J.J. Thomson. Since then, there has been an explosion in the discovery of new sub-atomic particles by experimental physicists and theoretical physicists have been busy explaining the nature of these new particles and their interactions.

In early 1960s development of the "Eightfold way" by Gell-Mann 8 led to the discovery of the quark model, which explained the multitude of new particles being discovered in cosmic ray and scattering experiments. In the mid-1960's the current model of the Electroweak Theory 911 was formulated by Glashow, Weinberg, and Salam. By the late 1970's, the theory of Electroweak interactions, the theory of the strong interactions known as QCD, and the flavor structure of the quarks and leptons were combined to form the Standard Model of particle physics. By probing the structure of the proton, experiments carried out at SLAC and CERN in 1974 confirmed the quark model 12, 13. Later experiments performed at CERN discovered the $\mathrm{W}$ and $\mathrm{Z}$ bosons 14,15 in 1983, confirming the Electroweak Theory.

In this chapter, the theoretical framework of the Standard Model, used to describe the elementary particles and their interactions is outlined in a manner similar to several other reviews 16 21]. In addition, several attractive extensions of the Standard Model are described. 


\subsection{The Standard Model}

The Standard Model of particle physics (SM) 11 is a theoretical model that describes the physical properties and dynamics of matter in terms of its fundamental constituents and their interactions. It uses a mathematical framework based on the symmetry group $S U(3)_{C} \times S U(2)_{L} \times U(1)_{Y}$, where each of the symmetries correspond to a fundamental force. The three fundamental forces are the the strong nuclear force (quantum chromodynamics), electromagnetic force (quantum electrodynamics), and the weak nuclear force. All three forces are mediated by gauge bosons.

The matter field of the SM are 12 spin $1 / 2$ particles that come in 3 generations. The 6 leptons are the electron $(\mathrm{e})$, the muon $(\mu)$, and the tau $(\tau)$, with one negative unit of electric charge, and three neutrinos $\left(\nu_{e}, \nu_{\mu}, \nu_{\tau}\right)$, that are electrically neutral. The 6 quarks are either "up-type", which are the up $(\mathrm{u})$, charm $(\mathrm{c})$, and top $(\mathrm{t})$ quarks that carry an electric charge of $+2 / 3$, or "down-type", which are the down (d), strange (s), and bottom (b) quarks that carry an electric charge of $-1 / 3$.

The summary of the known particles and force carrying gauge bosons can be seen in Fig. 2.1 .

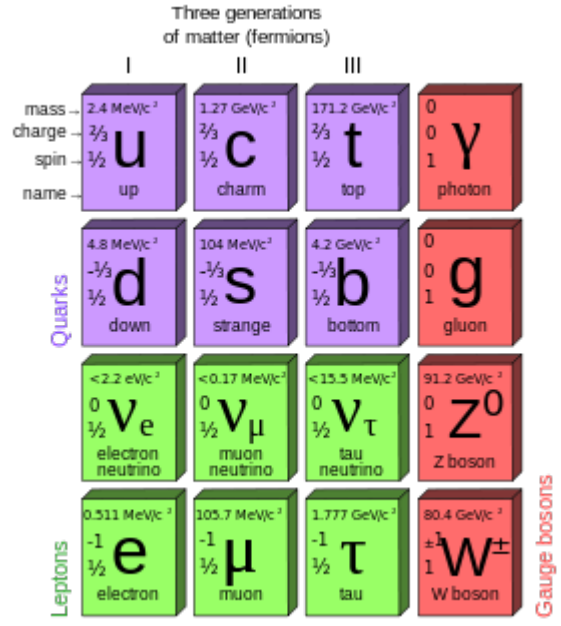

Figure 2.1: The known fundamental particles of nature.

\subsubsection{Quantum Chromodynamics}

Quantum chromodynamics (QCD) is a gauge field theory with local gauge symmetry $S U(3)_{C}$ that describes the strong nuclear force. The conserved quantum number of $S U(3)_{C}$ is referred to as color. The gauge fields that mediate the strong interaction correspond to the eight generators of the $S U(3)$ group and are referred to as gluons. The gluons are electrically neutral, but carry color charge and are therefore strongly self-interacting. The Lagrangian of QCD is [16: 


$$
\mathcal{L}=\sum_{q} \bar{\psi}_{q, a}\left(i \gamma^{\mu} \partial_{\mu} \delta_{a, b}-g_{s} \gamma^{\mu} t_{a b}^{C} G_{\mu}^{C}-m_{q} \delta_{a b}\right) \psi_{q, b}-\frac{1}{4} G_{\mu \nu}^{A} G^{A \mu \nu}
$$

The $\psi_{q, a}$ are spin-1/2 quark-field spinors for one of the six SM quarks with mass $m_{q}$, and the $\gamma^{\mu}$ are Dirac $\gamma$-matrices. The color-index $a$ runs from $a=1$ to the number of colors $n_{c}=3$. In the SM, each flavor of quark comes in three colors.

The $G_{\mu}^{C}$ correspond to the $n_{c}^{2}-1=8$ gluon fields. Gluons transform under the adjoint representation of the $S U(3)$ color group. The $t^{C}$ are the generators of the $S U(3)$ group and can be represented by eight $3 \times 3$ unitary matrices with determinant equals to 1, also known as Gell-Mann matrices. They describe how a gluon's interaction with a quark rotates the quark's color in $S U(3)$ space. The quantity $g_{s}$ is the QCD gauge-coupling constant, sometimes expressed as $\alpha_{s}=\frac{g_{s}^{2}}{4 \pi}$. The coupling constant $g_{s}$ is a free parameter of the theory, as are the quark masses $m_{q}$. Finally, the field tensor $G_{\mu \nu}^{A}$ is given by

$$
G_{\mu \nu}^{A}=\partial_{\mu} G_{\nu}^{A}-\partial_{\nu} G_{\mu}^{A}-g_{s} f_{A B C} G_{\mu}^{B} G_{\nu}^{C}
$$

where

$$
\left[t^{A}, t^{B}\right]=i f_{A B C} t^{C}
$$

An essential feature of QCD is that neither quarks nor gluons are observed as free particles 22 . Instead, quarks and anti quarks combine to form bound states referred to as hadrons. The reason for this feature begins with the fact that the coupling constant $\alpha_{s}$ of QCD runs with energy, as in QED, but with opposite sign. The energy dependence of $\alpha_{s}$ is given by:

$$
\alpha_{s}\left(Q^{2}\right)=\frac{\alpha_{s}\left(\mu^{2}\right)}{1+\left[\alpha_{s}\left(\mu^{2}\right) / 12 \pi\right]\left(11 n_{c}-2 n_{f}\right) \ln \left(Q^{2} / \mu^{2}\right)} \quad\left(Q^{2} \gg \mu^{2}\right),
$$

where $Q$ is the energy of the interaction, $\mu$ is the renormalization scale, and $n_{f}$ is the number of quark flavors. In the SM, $n_{f}=6$ and $n_{c}=3$, and therefore $\alpha_{s}$ grows large as $Q$ decreases, i.e. long-distance, and conversely $\alpha_{s}$ becomes small for short-distance interactions. This phenomena is called asymptotic freedom 23, 24]. The measured energy dependence of $\alpha_{s}$ can be seen in Fig. 2.2 .

At short distances, the smallness of $\alpha_{s}$ allows for calculations of QCD interactions to be performed perturbatively in terms of quasi-free quarks and gluons. At long distances (low energy) the QCD coupling strength becomes large, making QCD non-perturbative and difficult to calculate.

Eq. 2.4 can be rewritten in terms of an energy scale $\Lambda_{Q C D}$ as: 


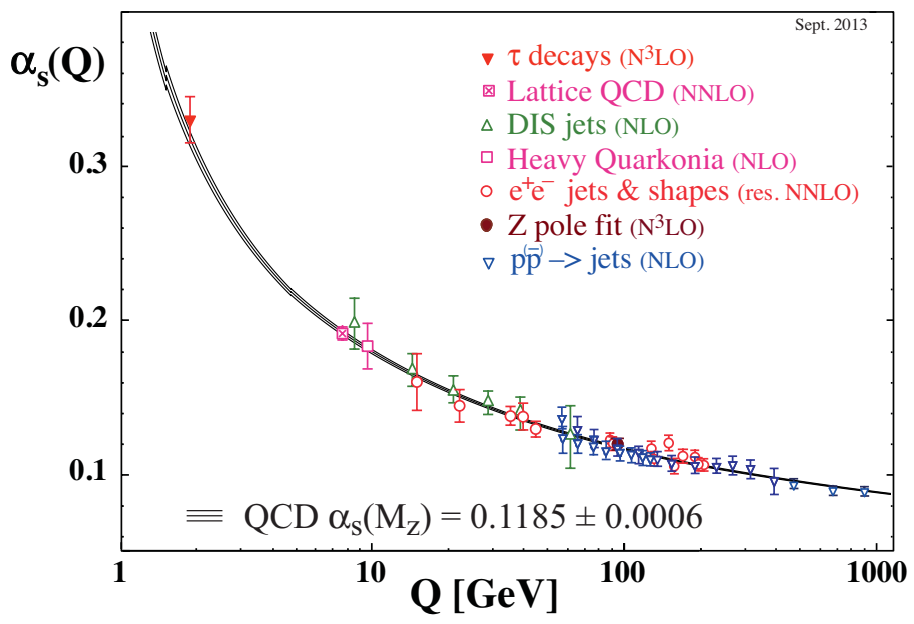

Figure 2.2: Summary of measurements of $\alpha_{s}$ as a function of the energy scale $Q 16$.

$$
\alpha_{s}\left(Q^{2}\right)=\frac{12 \pi}{\left(11 n_{c}-2 n_{f}\right) \ln \left(Q^{2} / \Lambda_{Q C D}^{2}\right)} \quad\left(Q^{2} \gg \Lambda_{Q C D}^{2}\right) .
$$

The energy scale $\Lambda_{Q C D}$ is known as the QCD scale and can be thought of as the energy boundary between quasi-free quarks and gluons and the bound states of hadrons. The QCD scale is therefore of the order of the lightest hadron masses, or approximately a few hundred $\mathrm{MeV}$ 16. The effective potential between two quarks, or a quark and an anti quark, can be calculated in perturbation theory and be shown to be attractive for color-singlet (i.e. $S U(3)$-invariant) configurations. Such configurations can be be formed by a quark and an anti-quark (called mesons), or by three quarks (called baryons).

\subsubsection{Electroweak Model}

The electromagnetic and weak nuclear forces are described by a unified gauge field theory with local gauge symmetry group $S U(2) \times U(1)$ first identified by Sheldon Glashow in 1961 25]. The electroweak Lagrangian for massless fermions and gauge bosons is given by:

$$
\begin{aligned}
\mathcal{L}_{E W}= & -\frac{1}{4} B_{\mu \nu} B^{\mu \nu}-\frac{1}{4} W_{\mu \nu}^{a} W_{a}^{\mu \nu}+\bar{L}_{i} i D_{\mu} \gamma^{\mu} L_{i}+\bar{\ell}_{i_{R}} i D_{\mu} \gamma^{\mu} \ell_{i_{R}} \\
& +\bar{Q}_{i} i D_{\mu} \gamma^{\mu} Q_{i}+\bar{u}_{i_{R}} i D_{\mu} \gamma^{\mu} u_{i_{R}}+\bar{d}_{i_{R}} i D_{\mu} \gamma^{\mu} d_{i_{R}} .
\end{aligned}
$$

In Eq. 2.6. $Q_{i}$ and $L_{i}$ are $S U(2)$ doublets consisting of the left-handed quark and lepton fields, respectively, for the $i^{t h}$ generation. The right-handed fields are $S U(2)$-singlets, i.e. they do not 
participate in the weak interactions. They include, for each generation $i$, the right-handed lepton fields $\ell_{i R}$, and the right-handed quark fields $u_{i R}$ and $d_{i R}$.

The three gauge fields $W_{\mu}^{1,2,3}$ are associated to the three generators of the $S U(2)$ symmetry group, which can be represented by three $2 \times 2$ matrices with the determinant equal to 1 , denoted by $\sigma^{a}$ and commonly referred to as the Pauli matrices. The gauge field $B_{\mu}$ is associated to the generator of the $U(1)$ symmetry group. The associated gauge fields are required to preserve the symmetry of the Langrangian under the local gauge transformations. The gauge coupling constants for the $S U(2)$ and $U(1)$ symmetries are denoted $g$ and $g^{\prime}$, respectively. The field-strength tensors corresponding to these gauge fields are given by:

$$
\begin{gathered}
W_{\mu \nu}^{a}=\partial_{\mu} W_{\nu}^{a}-\partial_{\nu} W_{\mu}^{a}-g \epsilon^{a b c} W_{\mu}^{b} W_{\nu}^{c} \\
B_{\mu \nu}=\partial_{\mu} B_{\nu}-\partial_{\nu} B_{\mu} .
\end{gathered}
$$

The gauge-covariant derivative, $D_{\mu}$, minimally couples the fermions to the gauge bosons and is given by:

$$
D_{\mu} \equiv \partial_{\mu}+i g \frac{\sigma^{a}}{2} W_{\mu}^{a}+i g^{\prime} \frac{Y}{2} B_{\mu}
$$

The gauge invariance requires the gauge fields to transform as:

$$
\begin{aligned}
\vec{W}_{\mu}(x) & \rightarrow \vec{W}_{\mu}(x)-\frac{1}{g} \partial_{\mu} \vec{\alpha}(x)-\vec{\alpha}(x) \times \vec{W}_{\mu}(x), \\
B_{\mu}(x) & \rightarrow B_{\mu}(x)-\frac{1}{g} \partial_{\mu} \beta(x),
\end{aligned}
$$

where $\alpha(x)$ and $\beta(x)$ are arbitrary functions of space-time coordinates called the transformation parameters.

The physical $W_{\mu}^{ \pm}, Z_{\mu}$, and photon $\left(A_{\mu}\right)$ fields are formed from linear combinations of the $W_{\mu}^{a}$ and $B_{\mu}$ fields:

$$
\begin{aligned}
W_{\mu}^{ \pm} & =\frac{1}{\sqrt{2}}\left(W_{\mu}^{1} \mp i W_{\mu}^{2}\right) ; \\
Z_{\mu} & =\cos \left(\theta_{W}\right) W_{\mu}^{3}-\sin \left(\theta_{W}\right) B_{\mu} ; \\
A_{\mu} & =\sin \left(\theta_{W}\right) W_{\mu}^{3}+\cos \left(\theta_{W}\right) B_{\mu},
\end{aligned}
$$


where $\theta_{W}$ is known as the weak mixing (or Weinberg) angle, which relates the coupling strengths $g$ and $g^{\prime}$ to the more familiar electromagnetic charge:

$$
g=\frac{e}{\sin \left(\theta_{W}\right)}, \quad \frac{g}{g^{\prime}}=\frac{\sin \left(\theta_{W}\right)}{\cos \left(\theta_{W}\right)} .
$$

The conserved quantum number of the $\mathrm{SU}(2)$ symmetry, referred to as the weak isospin, is denoted as $T_{3}$. The conserved quantum number for the $\mathrm{U}(1)$ symmetry, referred to as the weak hypercharge, is denoted by $Y=2\left(Q-T_{3}\right)$ where $Q$ is the electromagnetic charge in unites of $e$.

\subsubsection{Higgs Boson and Electroweak Symmetry Breaking}

The SM Lagrangian presented so far includes no mass terms, which is in clear contention with the observation that, except for the photon and gluons, the fundamental fermions and bosons are massive. An explicit mass term can be added which would be invariant under $S U(3)$ transformations. However, such a term would explicitly violate the $S U(2)$ symmetry because the left-handed component is an $S U(2)$ doublet whereas the right-handed component is an $S U(2)$ singlet.

The Higgs mechanism was proposed in 1964 26 29] as a way to explain how to generate masses for the vector gauge bosons without explicitly violating the $S U(2) \times U(1)$ gauge invariance of the Lagrangian. Instead, the symmetry is broken spontaneously, i.e. the Lagrangian is symmetric but its ground state is not. The same mechanism can also be used to explain the fermion masses.

The Higgs mechanism is applied to the SM by introducing an additional SU(2) doublet of complex scalar fields (4 components) with the weak hypercharge $Y=+1$ :

$$
\phi=\left(\begin{array}{c}
\phi^{+} \\
\phi^{0}
\end{array}\right)=\frac{1}{\sqrt{2}}\left(\begin{array}{c}
\phi_{1}+i \phi_{2} \\
\phi_{3}+i \phi_{4}
\end{array}\right), \quad Y_{\phi}=+1 .
$$

The SM Lagrangian also includes the standard kinetic term for a scalar field (using the same gauge covariant derivative $D_{\mu}$ as in Eq. 2.8), as well as a scalar field potential:

$$
\begin{gathered}
\mathcal{L}_{\phi}=\left(D_{\mu} \phi\right)^{\dagger}\left(D^{\mu} \phi\right)-V(\phi), \\
V(\phi)=\mu^{2} \phi^{\dagger} \phi+\lambda\left(\phi^{\dagger} \phi\right)^{2} .
\end{gathered}
$$

For all values of $\mu^{2}$ and $\lambda$, the Lagrangian of Eq. 2.13 respects the $S U(2) \times U(1)$ gauge symmetry. The value of the parameter $\lambda$ must be positive in order for the potential to have a minimum energy. 
The vacuum expectation value of $\phi$ maintains the symmetry of the Lagrangian when $\mu^{2}>0$ :

$$
\langle\phi\rangle_{0} \equiv\langle 0|\phi| 0\rangle=\left(\begin{array}{l}
0 \\
0
\end{array}\right)
$$

A visualization of this potential can be seen on the left side of Fig. 2.3 However, if $\mu^{2}<0$ (right side of Fig. 2.3, the vacuum expectation value $v$ is non-zero:

$$
\langle\phi\rangle_{0} \equiv\langle 0|\phi| 0\rangle=\left(\begin{array}{c}
0 \\
v / \sqrt{2}
\end{array}\right), \quad v \equiv \sqrt{\frac{-\mu^{2}}{\lambda}}
$$
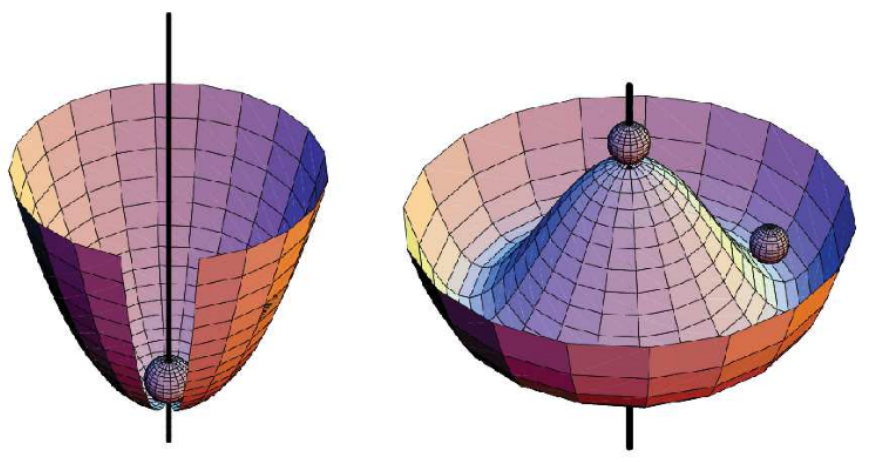

Figure 2.3: Potential $V(\phi)$ with $\mu^{2} \geq 0$ (left) and $\mu^{2}<0$ (right). From Ref. 30 .

It can be shown that the vacuum expectation value of the scalar field breaks the $S U(2)$ symmetry but maintains a new $U(1)$ symmetry, which is identified with electromagnetism. Therefore, the $W$ and $Z$ bosons will acquire a mass and the photon will remain massless. To see this, the scalar doublet is written as a first order expansion around the minimum of the scalar potential:

$$
\phi(x)=\left(\begin{array}{c}
\xi_{1}(x)+i \xi_{2}(x) \\
\frac{1}{\sqrt{2}}[v+H(x)]-i \xi_{3}(x)
\end{array}\right)=e^{i \frac{\sigma^{a}}{2 v} \xi_{a}(x)} \frac{1}{\sqrt{2}}\left(\begin{array}{c}
0 \\
v+H(x)
\end{array}\right)
$$

An $S U(2)$ gauge transformation, referred to as the unitary gauge transformation, can be used to eliminate the phase factor in Eq. 2.16. In the unitary gauge the kinetic term of the Lagrangian in Eq. 2.13 can be expanded as:

$$
\left(D_{\mu} \phi\right)^{\dagger}\left(D^{\mu} \phi\right) \rightarrow \frac{1}{2} \partial_{\mu} H \partial^{\mu} H+(v+H)^{2}\left\{\frac{g^{2}}{4} W_{\mu}^{\dagger} W^{\mu}+\frac{g^{2}}{8 \cos ^{2} \theta_{W}} Z_{\mu} Z^{\mu}\right\}
$$

Therefore, after breaking of the $S U(2) \times U(1)$ symmetry, the mass terms for the $W$ and $Z$ gauge 
bosons at leading order are:

$$
m_{W}^{2}=\frac{v^{2} g^{2}}{4}, \quad \frac{1}{2} m_{Z}^{2}=\frac{v^{2} g^{2}}{8 \cos ^{2} \theta_{W}} .
$$

Mass terms for the SM fermions can be generated using the same complex scalar doublet. Several $S U(2) \times U(1)$-invariant interaction terms, referred to as a Yukawa terms, can be added to the scalar Lagrangian:

$$
\mathcal{L}_{F}=-y_{\ell_{i}} \bar{L}_{i} \phi \ell_{R_{i}}-y_{d_{i}} \bar{Q}_{i} \phi d_{R_{i}}-y_{u_{i}} \bar{Q}_{i} \tilde{\phi} u_{R_{i}}+\text { h.c. },
$$

where $\tilde{\phi}=i \sigma^{2} \phi^{*}$ and the constants $y_{f_{i}}$ are the free parameters of the model. After electroweak symmetry breaking these terms become:

$$
\mathcal{L}_{F}=-\frac{1}{\sqrt{2}} y_{\ell_{i}} \bar{\ell}_{L_{i}}(v+H) \ell_{R_{i}}-\frac{1}{\sqrt{2}} y_{q_{i}} q_{L_{i}}(v+H) q_{R_{i}}
$$

such that the masses of the fermions are $m_{f}=y_{f} v / \sqrt{2}$. There are also terms that give $H \bar{f}_{L} f_{R}$ interactions with coupling strengths proportional to the fermion masses. It is interesting to note that the neutrinos remain massless.

Finally, the scalar potential in Eq. 2.13 gives rise to mass and self-interaction terms for the physical Higgs boson. After electroweak symmetry breaking, the scalar potential can be expanded as:

$$
\mathcal{L}_{V}=-\frac{1}{2} \lambda v^{2}(v+H)^{2}+\frac{1}{4} \lambda(v+H)^{4},
$$

which gives the mass of the Higgs boson at first order to be $m_{H}^{2}=2 \lambda v^{2}$.

The main production mechanisms of the Higgs boson at high-energy hadron colliders are gluon fusion, vector-boson fusion, associated production with a gauge boson, and associated production with top quarks. Figure 2.4 shows the Feynman diagrams for these Higgs boson production processes.

At the Large Hadron Collider (LHC), the dominant Higgs boson production mechanism is the gluon fusion process, mediated by the exchange of a virtual, heavy top quark 31. Contributions from lighter quarks propagating in the loop are sub-leading since the Higgs boson coupling to fermions is proportional to the fermion's mass. The production cross section is sensitive to higher-order QCD corrections. The next-to-leading-order (NLO) corrections increase the leading-order prediction by 


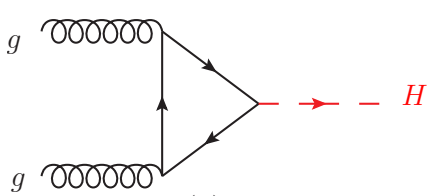

(a)

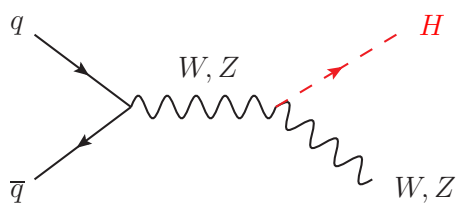

(c)
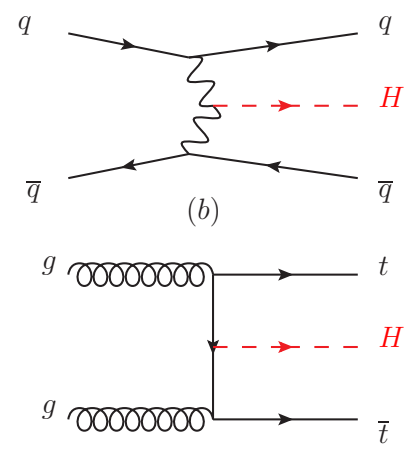

(d)

Figure 2.4: Feynman diagrams for the Higgs boson production in (a) gluon fusion, (b) vector-boson fusion, (c) associated production with a gauge boson, and (d) associated production with top quarks. From Ref. 16

about $80 \%$. Additionally, the next-to-NLO (NNLO) corrections further increase the cross section by about $20 \%$, and NLO electroweak contributions further enhance the cross section by about $5 \%$. Figure 2.5 shows the cross section for the production of a SM Higgs boson as a function of the center-of-mass energy for proton-proton collisions, along with the theoretical uncertainties.

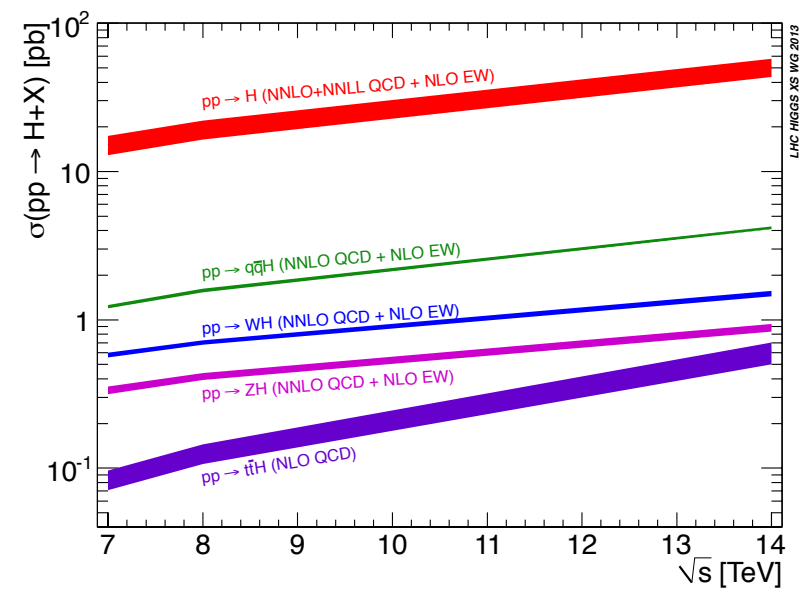

Figure 2.5: The SM Higgs boson production cross sections, as a function of the center-of-mass energy, for pp collisions. The theoretical uncertainties are indicated as a band. From Ref. 16].

\subsection{Beyond the Standard Model}

Although, the SM describes remarkably well everything we see in the laboratory experiments (the gravitational force aside), it is not an entirely satisfactory theory to explain all that we see in nature. For example, in the SM, the SM Higgs sector is highly unnatural. Higher-order corrections to the 
Higgs boson mass are proportional to an arbitrary cut-off scale and are referred to as "quadratic divergences". If the the cut-off scale is high, the corrections become large compared with the on-shell mass of the Higgs boson. This is often called the "fine-tuning" or "hierarchy" problem of the SM.

The fine-tuning problem of the SM can be resolved if there exist new particles beyond the SM whose masses define the cut-off scale. If these new particles are related to those of the SM through a new symmetry, the quadratic divergences from the SM particles can be partially or completely cancelled. One such class of theories is referred to as "supersymmetry" or SUSY for short. In SUSY models, the SM is extended to include an additional space-time symmetry between bosons and fermions. Due to the cancellation between the diagrams involving SM particles and the analogous diagrams involving their superpartners (i.e. SUSY particles), the quadratic divergences to the Higgs boson mass are cancelled to all orders in perturbation theory. More details about SUSY theories will be given in Section 2.2.1.

Supersymmetry is not the only extension to the SM that can resolve the fine-tuning problem. The problem can be resolved without invoking supersymmetry if there exist additional spatial dimensions. In extra dimensional models, the cut-off scale is the true Planck scale, which is much lower than the apparent Planck scale $M_{p} \sim 10^{16} \mathrm{TeV}$, if the size of the extra dimensions is large. If the size of the extra dimensions are $\sim 0.1-1 \mathrm{~mm}$, the true Planck scale should be $\sim \mathrm{TeV}$, which can resolve the fine-tuning problem. New particles, which are Kaluza-Klein excitations of SM particles, are predicted with masses $\sim \mathrm{TeV} 32$.

A more direct indication of new physics is the strong evidence for the existence of dark matter in the universe. Cosmological observations such as the rotation curves of stars in the galaxy and the effects of gravitational lensing strongly favor the existence of a stable, neutral particle that accounts for roughly $22 \%$ of the total energy of the universe. The SM, however, provides no explanation for the existence of dark matter. The SM also can not explain the masses of the neutrinos. Neutrino masses are required to explain the observed phenomena of neutrino oscillations [33, and seemingly require the existence right-handed neutrino fields, which neither have been observed, nor exist in the SM. Finally, the SM does not explain the large asymmetry between the matter and anti matter required to produce all of the observed baryonic matter in the universe.

\subsubsection{Supersymmetry}

Supersymmetry (SUSY) 34 40] is a generalization of the space-time symmetries of quantum field theory relating fermions to bosons. SUSY predicts the existence of a "superpartner" particle to each 
SM particle, differing by $1 / 2$ unit of spin. If SUSY were an exact symmetry of nature, then SM particles and their superpartners would have the same mass. Since this has been experimentally excluded, SUSY must instead be a broken symmetry. The SUSY breaking can be accomplished through spontaneous symmetry breaking, or by adding "soft-breaking" 41 terms to the Lagrangian, which explicitly break SUSY, but still allow for a resolution of the fine tuning problem of the SM.

The minimal supersymmetric extension of the SM (MSSM) contains two Higgs doublets, instead of just one, along with the superpartner particles [42]. A particle and its superpartner together form what is referred to as a "supermultiplet". The field content of the MSSM and the gauge quantum numbers of the MSSM supermultiplets are shown in Figure 2.6.

\begin{tabular}{|c|c|c|c|c|c|c|}
\hline \multicolumn{7}{|c|}{ Field Content of the MSSM } \\
\hline $\begin{array}{c}\text { Super- } \\
\text { multiplets }\end{array}$ & $\begin{array}{c}\text { Super- } \\
\text { field }\end{array}$ & $\begin{array}{c}\text { Bosonic } \\
\text { fields }\end{array}$ & $\begin{array}{c}\text { Fermionic } \\
\text { partners }\end{array}$ & $\mathrm{SU}(3)$ & $\mathrm{SU}(2)$ & $\mathrm{U}(1)$ \\
\hline gluon/gluino & $\widehat{V}_{8}$ & $g$ & $\widetilde{g}$ & 8 & 1 & 0 \\
gauge/ & $\widehat{V}$ & $W^{ \pm}, W^{0}$ & $\widetilde{W}^{ \pm}, \widetilde{W}^{0}$ & 1 & 3 & 0 \\
gaugino & $\widehat{V}^{\prime}$ & $B$ & $\widetilde{B}$ & 1 & 1 & 0 \\
\hline slepton/ & $\widehat{L}$ & $\left(\widetilde{\nu}_{L}, \widetilde{e}_{L}^{-}\right)$ & $\left(\nu, e^{-}\right)_{L}$ & 1 & 2 & -1 \\
lepton & $\widehat{E}^{c}$ & $\tilde{e}_{R}^{-}$ & $e_{R}^{-}$ & 1 & 1 & -2 \\
\hline squark/ & $\widehat{Q}$ & $\left(\widetilde{u}_{L}, \widetilde{d}_{L}\right)$ & $(u, d)_{L}$ & 3 & 2 & $1 / 3$ \\
quark & $\widehat{U}^{c}$ & $\widetilde{u}_{R}$ & $u_{R}$ & 3 & 1 & $4 / 3$ \\
& $\widehat{D}^{c}$ & $\widetilde{d}_{R}$ & $d_{R}$ & 3 & 1 & $-2 / 3$ \\
\hline Higgs/ & $\widehat{H}_{d}$ & $\left(H_{d}^{0}, H_{d}^{-}\right)$ & $\left(\widetilde{H}_{d}^{0}, \widetilde{H}_{d}^{-}\right)$ & 1 & 2 & -1 \\
higgsino & $\widehat{H}_{u}$ & $\left(H_{u}^{+}, H_{u}^{0}\right)$ & $\left(\widetilde{H}_{u}^{+}, \widetilde{H}_{u}^{0}\right)$ & 1 & 2 & 1 \\
\hline
\end{tabular}

Figure 2.6: The field content of the MSSM.

The MSSM possesses a discrete symmetry referred to as R-parity, where $\mathrm{R}=(-1)^{-3(B-L)+2 S}$ where B and L are the baryon and lepton numbers for a particle of spin S [43]. The ordinary SM particles have $R=+1$, while their superpartners have $R=-1$. The phenomenological consequence of the R-parity conservation is that in a scattering experiment involving SM particles in the initial state, any SUSY particles must be produced in pairs. These particles are unstable and decay into lighter states. Consequently, the R-parity conservation requires the lightest SUSY particle (LSP) to be stable, the LSP will be produced at the end of all SUSY decay chains. If the LSP is electrically neutral, it is a very attractive candidate for dark matter.

\subsubsection{A Simplified Model for the Low-Scale SUSY Breaking Scenerio}

A model-independent consequence of the spontaneous breaking of global SUSY is the existence of a Goldstone fermion, called the goldstino. The goldstino would then be the LSP, and could play an important role in SUSY phenomenology. However, if SUSY is a local symmetry, then the theory 
must incorporate gravity. In such a theory of "supergravity" [44], the spin-1/2 goldstino is absorbed by the spin $3 / 2$ gravitino, giving the gravitino a mass $m_{3 / 2}=f /\left(\sqrt{3} M_{p}\right)$. When the SUSY breaking scale $\sqrt{f}$ is $\mathcal{O}(\mathrm{TeV})$, the gravitino is approximately massless $\left(m_{3 / 2} \sim 10^{-3} \mathrm{eV}\right)$ and, due to the supersymmetric equivalence theorem [45], can be replaced by its goldstino components. The equivalence theorem states that the interactions of the spin-1/2 gravitino (whose properties approximate those of the goldstino) dominate those of the spin-3/2 gravitino. Furthermore, gravitational effects can be neglected and SUSY can be treated as an approximate global symmetry. In the case where the goldstino $G$ is the LSP, R-parity is preserved and the lightest neutralino $\chi_{1}^{0}$ is the next-to-LSP (NLSP), whose only relevant decay mode is into a goldstino and a photon.

The effective new physics Lagrangian for this model can be written as:

$$
\mathcal{L}_{N P}=\frac{m^{2}}{\sqrt{2} f}\left[g_{h \chi} h \chi_{1}^{0} G+\frac{g_{\chi \gamma}}{m} G \sigma^{\mu \nu} F_{\mu \nu} \chi_{1}^{0}+\frac{g_{\chi Z^{1}}}{m} G \sigma^{\mu \nu} Z_{\mu \nu} \chi_{1}^{0}+g_{\chi Z^{2}} \bar{G} \bar{\sigma}^{\mu} Z_{\mu} \chi_{1}^{0}+h . c .\right] .
$$

Here, $F_{\mu \nu}$ and $Z_{\mu \nu}$ are the photon and $Z$ boson field strengths, $h$ is the Higgs boson, $\chi_{1}^{0}$ is the NLSP neutralino, and $\mathrm{G}$ is the goldstino. The $g$ 's are the dimensionless couplings and $\sigma^{i}$ are the Pauli matrices. The mass scales $\sqrt{f}$ and $m$ can be interpreted as the SUSY breaking scale and a scale related to the soft parameters, respectively. The new physics Lagrangian is only relevant when $\sqrt{f}$ is close $m$. Since $m$ is also related to $m_{h}$ and $m_{\chi}$, it is expected to be of the order of the EW scale and therefore $\sqrt{f}$ should be $\mathcal{O}(\mathrm{TeV})$.

For $m_{\chi}<m_{h} / 2$, the Higgs particle can decay into two neutralinos, each of which subsequently decays promptly into a photon and a goldstino giving rise to a final-state signature consisting of two photons and missing transverse energy. When $m_{h} / 2<m_{\chi}<m_{h}$, the decay of a SM-like Higgs boson $h$ into a goldstino and an NLSP neutralino is the dominant BSM decay mode and is the process studied in this thesis. The neutralino subsequently decays into a goldstino and a photon, the full production and decay chain being $g g \rightarrow h \rightarrow \chi_{1}^{0} G \rightarrow \gamma G G$. This process gives rise to a final-state signature consisting of a single photon and missing transverse energy. The partial width of this decay mode is given as:

$$
\Gamma\left(h \rightarrow \chi_{1}^{0} G\right)=\frac{m_{h}}{16 \pi} \frac{g_{h \chi}^{2} m^{4}}{f^{2}}\left(1-\frac{m_{\chi}^{2}}{m_{h}^{2}}\right)^{2}
$$

and the decay of the neutralino into a goldstino and photon has a partial width: 


$$
\Gamma\left(\chi_{1}^{0} \rightarrow \gamma G\right)=\frac{m_{\chi}^{3}}{16 \pi} \frac{g_{\chi \gamma}^{2} m^{2}}{f^{2}}
$$

The decay length of the neutralino is given by:

$$
L_{\chi}=\frac{1}{g_{\chi \gamma}^{2}} \frac{(100 \mathrm{GeV})^{5}}{m_{\chi}^{3} m^{2}}\left(\frac{\sqrt{f}}{1 \mathrm{TeV}}\right)^{4} \sqrt{\left(\frac{E^{2}}{m_{\chi}^{2}}-1\right)} \times 10^{-10} \mathrm{~cm} .
$$

One can always choose relevant parameters such that the neutralino decays promptly. 


\section{Chapter 3}

\section{Experimental Apparatus}

The Large Hadron Collider (LHC) 46 is the world's largest high-energy particle accelerator and collider, located at the European Center for Nuclear Research (CERN) near Geneva, Switzerland. The Compact Muon Solenoid (CMS) Experiment is a general-purpose detector that measures the properties of particles produced from pp and heavy-ion collisions at the LHC. This Chapter has the technical information explaining the design and the performance of the LHC and the CMS.

\subsection{The Large Hadron Collider}

The LHC is a two-ring superconducting hadron accelerator and it is designed to collide counterrotating proton beams with a centre-of-mass energy of $14 \mathrm{TeV}$ (7 TeV per beam) and luminosity of $10^{34} \mathrm{~cm}^{-2} \mathrm{~s}^{-1}$. It can also collide heavy $(\mathrm{Pb})$ ions with an energy of $2.8 \mathrm{TeV}$ per nucleon and a peak luminosity of $10^{27} \mathrm{~cm}^{-2} \mathrm{~s}^{-1}$.

Before reaching the final design energy of $7 \mathrm{TeV}$ per beam within the LHC protons that are extracted from hydrogen atoms by stripping the electrons with a large electric field, are first accelerated to $450 \mathrm{GeV}$ in the pre-accelerator facilities at CERN. These increasingly energetic linear accelerators and storage rings are known as the LHC injector chain [47], which consists of the Linac2, the Proton Synchrotron Booster (PSB), the Proton Synchrotron (PS), and the Super Proton Synchrotron (SPS). In this injector chain, the protons are accelerated to $50 \mathrm{MeV}, 1.4 \mathrm{GeV}, 25 \mathrm{GeV}$, and $450 \mathrm{GeV}$ respectively. Schematics of the CERN accelerator complex can be seen in Fig. 3.1 48 .

The LHC has two high-luminosity experiments, ATLAS [49] and CMS [50, both aiming at a peak luminosity of $\mathrm{L}=10^{34} \mathrm{~cm}^{-2} s^{-1}$ for proton operation. There are also two low-luminosity experiments: $\mathrm{LHCb} 51$ for B-physics, aiming at a peak luminosity of $\mathrm{L}=10^{32} \mathrm{~cm}^{-2} \mathrm{~s}^{-1}$ and a dedicated heavy- 


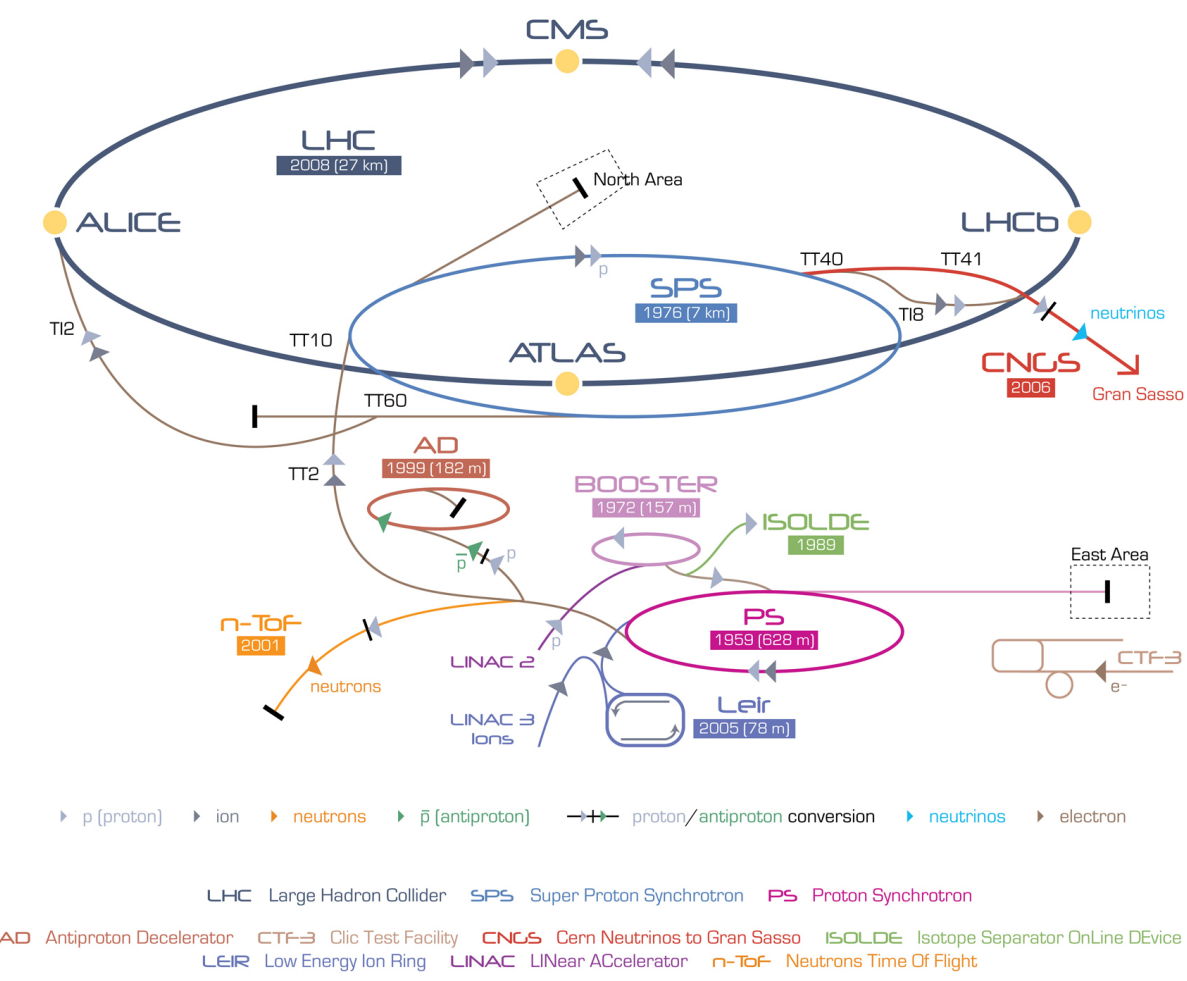

Figure 3.1: A schematic drawing of the CERN accelerator complex. From Ref. 48.

ion experiment, ALICE [52], aiming at a peak luminosity of $\mathrm{L}=10^{27} \mathrm{~cm}^{-2} \mathrm{~s}^{-1}$ for lead-lead ion operation. The positioning of these experiments can also be seen in Fig. 3.1 .

The number of events generated per second in the LHC can be written as:

$$
N_{\text {event }}=L \sigma_{\text {event }},
$$

where $\sigma_{\text {event }}$ is the cross section for the event and $L$ is the machine instantaneous luminosity. As seen, $\mathrm{L}$ is proportional to the number of events per second and thus has to be maximized. However it has dependency on the beam parameters. For a Gaussian beam distribution the luminosity can be written as:

$$
L=\frac{N_{b}^{2} n_{b} f_{r e v} \gamma_{r}}{4 \pi \epsilon_{n} \beta^{*}} F
$$

where $N_{b}$ is the number of particles per bunch, $n_{b}$ is the number of bunches per beam, $f_{\text {rev }}$ is the revolution frequency, $\gamma_{r}$ is the relativistic gamma factor $\left(\gamma_{r}=E_{p} / m_{p} c^{2}\right), \epsilon_{n}$ is the normalized transverse beam emittance which is a measure for the average spread of particle coordinates in position and momentum phase space, and $\beta^{*}$ is the beta function that describes the beam size at 
the collision point. The geometrical factor $F$ accounts for the reduction due to the small crossing angle at the interaction point. The peak luminosity and the energy of the LHC are limited by:

- the beam emittance, which has to fit into the small aperture of the superconducting LHC magnets;

- the thermal energy produced by synchrotron radiation, which must be absorbed by the cryogenic system (limits the total intensity $n_{b} \cdot N_{b}$ );

- the beam-beam effect causing a spread in betatron tunes (number of oscillations of particles about their stable orbits) when the beams are colliding has to be kept below a certain limit (limits the transverse beam brightness $N_{b} / \epsilon_{n}$ );

- the space-charge limit in the injectors (scales with $N_{b} / \epsilon_{n}$ ).

LHC has delivered $p p$ collisions at a reduced center of mass energy of $\sqrt{s}=7 \mathrm{TeV}$ during 2010 and 2011 , and of $\sqrt{s}=8 \mathrm{TeV}$ during 2012. The bunch spacing was fixed to $50 \mathrm{~ns}$ with a maximum of 1380 bunches and $10^{11}$ protons per bunch. The peak instantaneous luminosity achieved in 2012 was 7.67 $\times 10^{33} \mathrm{~cm}^{-2} \mathrm{~s}^{-1}[53$. Figure 3.2 shows the cumulative distributions of integrated luminosity versus time for 2011 and 2012 delivered by the LHC accelerator and recorded by the CMS experiment.
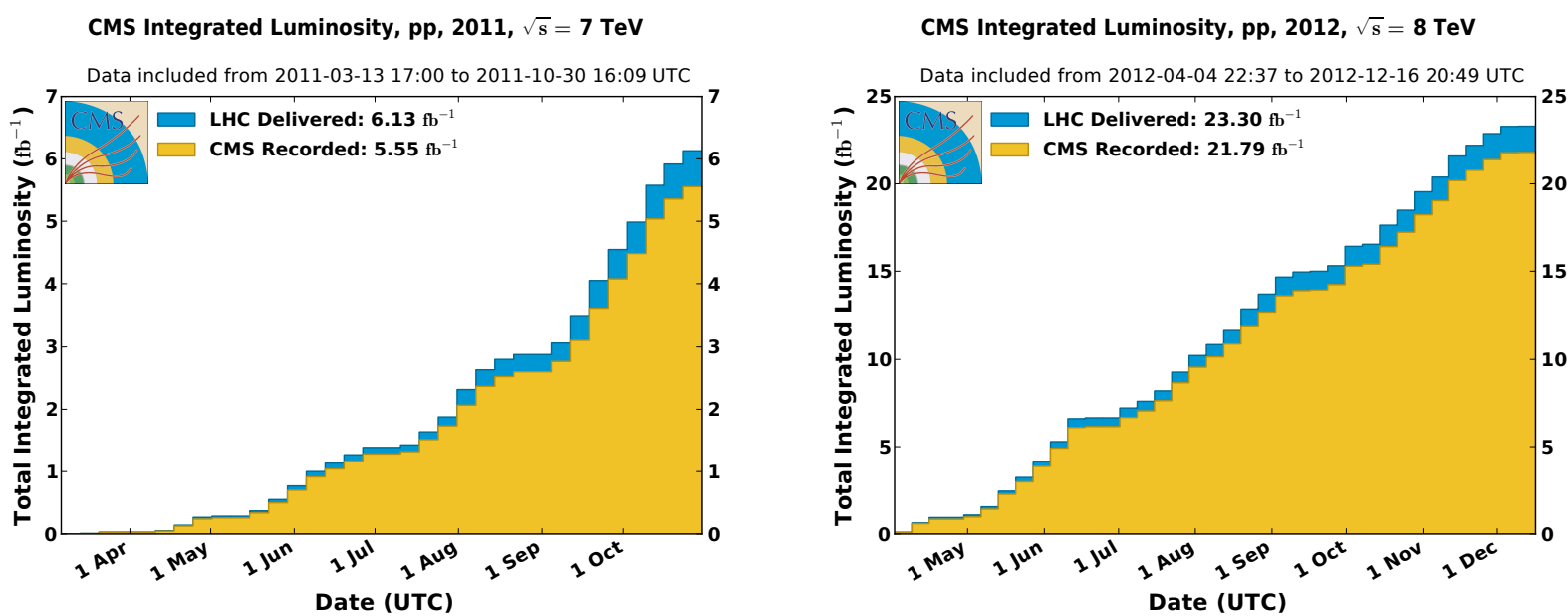

Figure 3.2: Integrated luminosity delivered by the LHC to the CMS experiment during the 2011 run at $\sqrt{s}=7 \mathrm{TeV}$ (left) and 2012 run at $\sqrt{s}=8 \mathrm{TeV}$ (left). From Ref. 53 


\subsection{The CMS Experiment}

The CMS experiment 54 is one of the two multipurpose detectors at the LHC . Conceptual design of the CMS experiment was geared towards the detection of the SM Higgs boson, which led to an excellent reconstruction efficiencies and energy resolution for electrons, muons, photons, and hadrons (mesons or baryons) [55. The requirements for the CMS detector to achieve the goals of the physics program can be summarized as:

- Good muon identification and momentum resolution over a wide range of momenta and angles; good dimuon mass resolution $(\approx 1 \%$ at $100 \mathrm{GeV})$, and the ability to determine unambiguously the charge of muons with $\mathrm{p}<1 \mathrm{TeV}$.

- Good charged-particle momentum resolution and reconstruction efficiency in the inner tracker. Efficient triggering and offline tagging of $\tau$ leptons and b-jets, requiring pixel detectors close to the interaction region.

- Good electromagnetic energy resolution, good diphoton and dielectron mass resolution $(\approx 1 \%$ at $100 \mathrm{GeV}$ ), wide geometric coverage, $\pi^{0}$ rejection, and efficient photon and lepton isolation at high luminosities.

- Good missing transverse energy and dijet mass resolution, requiring hadron calorimeters with a large hermetic geometrical coverage and with fine lateral segmentation.

The main feature of the CMS apparatus is a $3.8 \mathrm{~T}$ superconducting solenoid magnet with a 6 $\mathrm{m}$ internal diameter. Within the field volume are a silicion pixel and strip tracker, a lead tungstate crystal electromagnetic calorimeter (ECAL), and a brass/scintillator hadron calorimeter (HCAL). The identification and the measurement of muons is performed by a separate muon system of gasizonization detectors embedded in the steel flux-return yoke of the magnet. A diagram of the CMS detector can be see in Fig. 3.3 [56].

CMS uses a right-handed coordinate system with the origin at the collision point inside the experiment. The $x$ axis points inward to the center of the LHC ring, the $y$ axis points upward (perpendicular to the plane of the LHC ring), and the $z$ axis along the counter clockwise-beam direction. The radial distance $r$ is measured outwards from origin and the transverse components of particle momenta lies in the $x-y$ plane. The polar angle $\theta$ is measured from the positive $z$ axis and the azimuthal angle $\phi$ is measured in the $x-y$ plane. For convenience, a new quantity, the pseudorapidity, is defined as $\eta \equiv-\ln (\tan (\theta / 2))$, for which the particle flux at hadron colliders is approximately constant. 


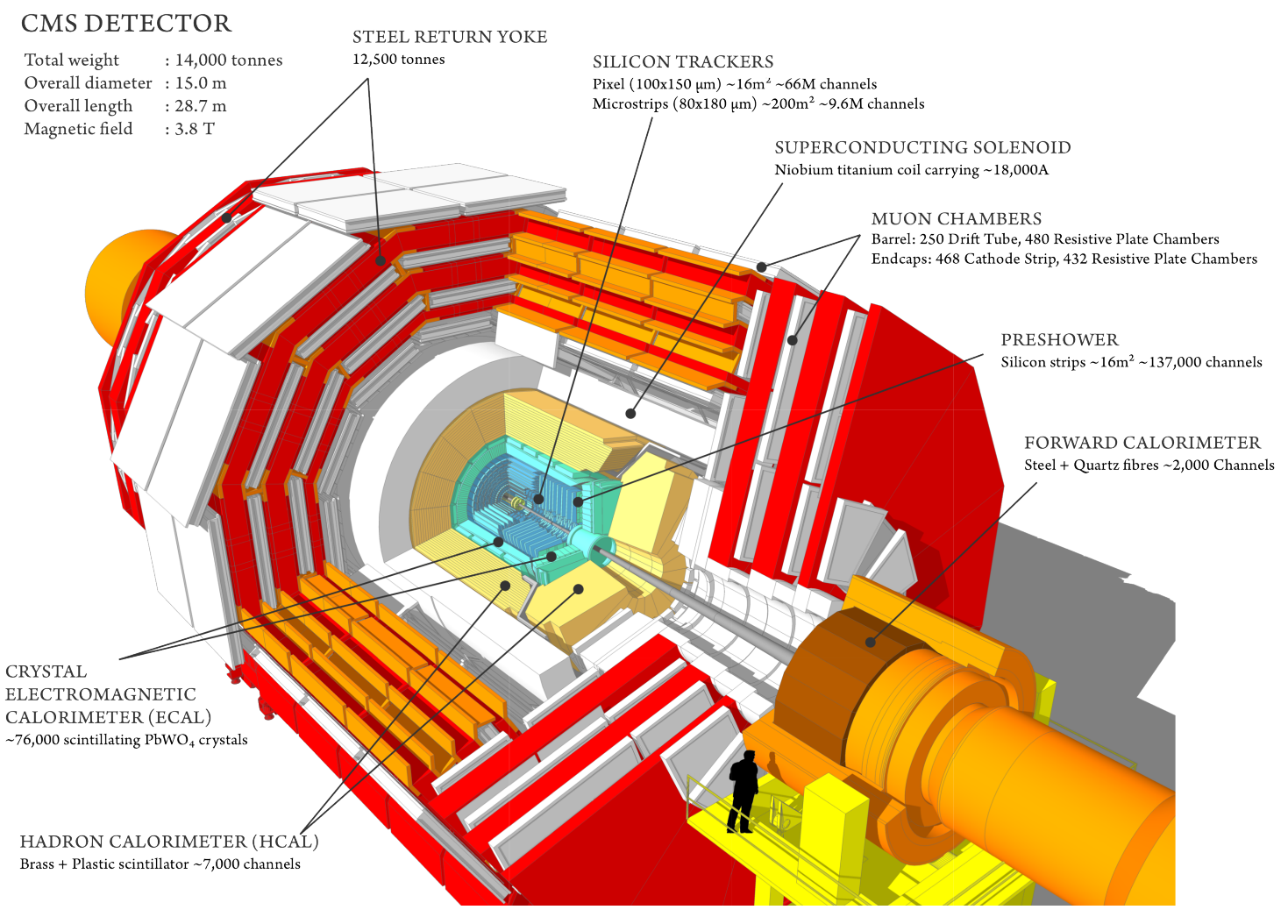

Figure 3.3: A perspective view of the CMS experiment. From Ref. 56. 


\subsubsection{CMS Tracking System}

To achieve the scientific goals of CMS, a good tracking system with excellent performance over a wide range of momenta is required. Good momentum resolution up to $1 \mathrm{TeV}$ is needed for searches for high-mass dilepton resonances where efficient reconstruction of tracks with $100 \mathrm{MeV}$ momentum is needed for to obtain optimum jet energy resolution for particle-flow techniques [57]. Precise measurement of the positions of primary pp interaction vertices as well as identification of b-quark jets requires excellent impact parameter resolution 58.

The CMS tracker [59] was designed with the above requirements in mind and has a cylindrical volume with a 5.8-meter length and 2.5-meter diameter inside a $3.8 \mathrm{~T}$ magnetic field. It consists of a large silicon strip tracker and a small silicon pixel tracker. A schematic drawing of the CMS tracker is shown in Fig. 3.4

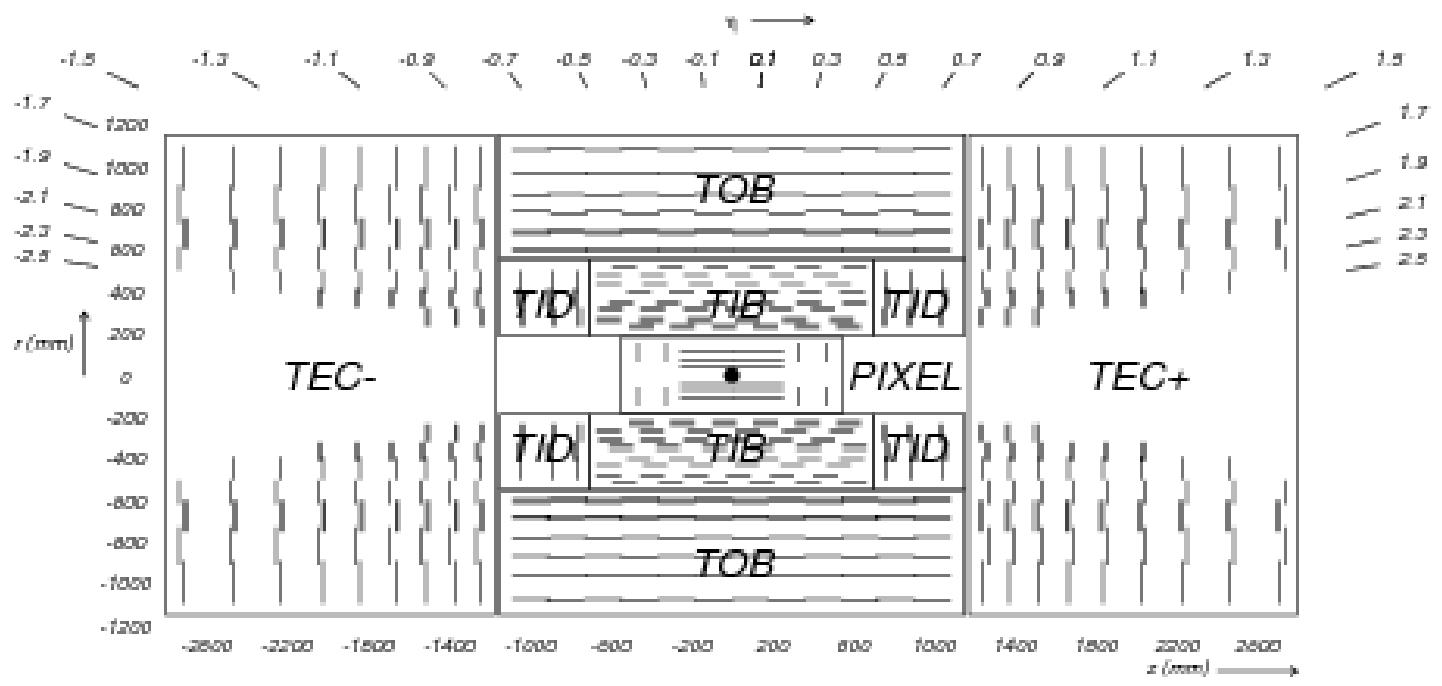

Figure 3.4: Schematic cross section through the CMS tracker in the $r-z$ plane. From Ref. 54.

The silicon pixel tracker is arranged in three barrel layers and two disks at each end. It provides three-dimensional (3-D) position measurements of the hits arising from the interaction of charged particles with spatial resolution in the range of $15-20 \mu \mathrm{m}$. It is compromised of 66 million pixels and covers an area of about $1 \mathrm{~m}^{2}$.

The silicon pixel detector is surrounded by the silicon strip tracker that is organized in total of ten barrel layers with twelve disks at each end. Silicon strip detector has 9.3 million strips in 15148 silicon modules with a total silicon surface area of $198 \mathrm{~m}^{2}$, and is composed of four subsystems. The Tracker Inner Barrel (TIB) and Disks (TID) provide position measurements in $r-\phi$ with a resolution 
in the range of $13-38 \mu \mathrm{m}$ through four barrel layers, with three disks at each end. The Tracker Outer Barrel (TOB) consists of six barrel layers. The resolution on the position measurements in $r-\phi$ is approximately $18-47 \mu \mathrm{m}$. The Tracker End Caps (TEC) are composed of nine disks with the resolution range similar to that of TOB.

The tracks in the CMS are seeded by the hits in the tracker detector. The compatible hits are added to update the trajectory until either the detector boundary is reached, or no additional compatible hits can be found. The collection of the hits are then used to obtain the best estimate of the track parameters. However, material within the tracking volume affects the overall event topology and reconstruction due to electron bremsstrahlung, photon conversions, and nuclear interactions. Therefore it is crucial to estimate the amount of material of the CMS tracker. The simulation describes the tracker material budget with $10 \%$ accuracy 60 . The total amount of material in the CMS tracker, both in units of radiation lengths and nuclear interaction lengths, as estimated from simulation and shown in Fig 3.5 . It increases from $0.4 X_{0}$ at $|\eta| \approx 0$ to about $1.8 X_{0}$ at $|\eta| \approx 1.4$, before decreasing to about $1 X_{0}$ at $|\eta| \approx 2.5$.
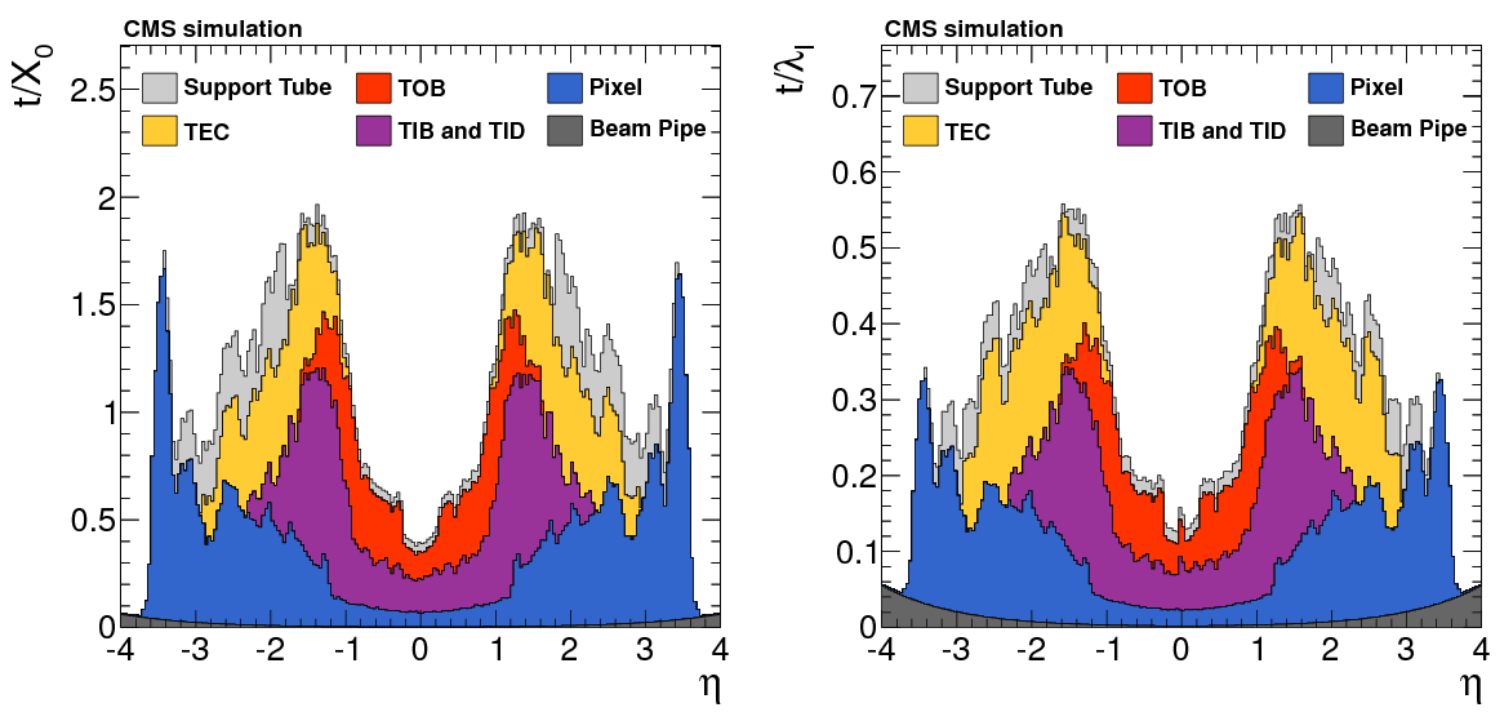

Figure 3.5: Total thickness of the tracker material traversed by a particle produced at the nominal interaction point, as a function of pseudorapidity, expressed in units of radiation length (left) and nuclear interaction length(right). From Ref. [59].

\subsubsection{CMS Electromagnetic Calorimeter}

The electromagnetic calorimeter (ECAL) is a homogeneous and hermetic calorimeter made of 75848 lead tungstate $\left(\mathrm{PbWO}_{4}\right)$ scintillating crystals. In order to fulfill the scientific goals of CMS, the 
ECAL was designed to have high resolution for e $/ \gamma$ objects. To achieve this, the ECAL was positioned inside the CMS magnet, to lower the amount of energy loss due to the material upstream. This lead to the design of the compact, fine lateraly granular ECAL using $\mathrm{PbWO}_{4}$ crystals with high density $\left(8.28 \mathrm{~g} / \mathrm{cm}^{3}\right)$, short radiation length $(8.9 \mathrm{~mm})$, and small Molière radius $(23 \mathrm{~mm})$. The total amount of material between the interaction point and the ECAL, in terms of radiation lengths $\left(X_{0}\right)$, raises from $0.4 X_{0}$ close to $\eta=0$ to almost $2 X_{0}$ near $|\eta|=1.4$, before falling to about $1.3 X_{0}$ around $|\eta|$ $=2.5$. Therefore the resolution of the ECAL depends on whether the electron or photon undergoes bremsstrahlung, the transverse momentum of the object, and also on $\eta$.

A layout of the ECAL can be seen in Fig. 3.6.

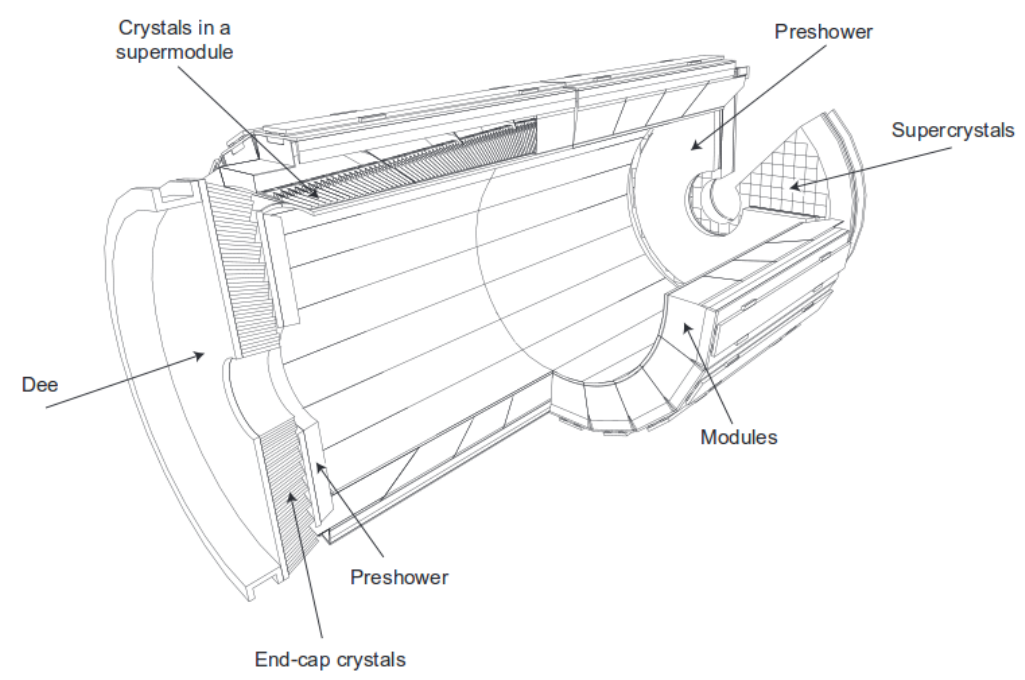

Figure 3.6: A schematic design of the CMS ECAL. From Ref. 54.

The ECAL central barrel (EB) covers $|\eta|<1.48$ with the internal surface at a radius of $1290 \mathrm{~mm}$. It is made of 61200 trapezoidal crystals. Each crystal has a frontal area of approximately $22 \times 22$ $\mathrm{mm}^{2}$ and a length of $230 \mathrm{~mm}\left(25.8 X_{0}\right)$, which lead to a granularity of 0.0174 in $\eta$ and $\phi$. Each half-barrel consists of 18 barrel supermodules each positioned at every $20^{\circ}$ in $\phi$ each containing 1700 crystals. Avalanche photodiodes (APDs) are used as photodetectors in the ECAL barrel.

The endcaps (EE) cover $1.48<|\eta|<3.00$ and are located at $|z|>3154 \mathrm{~mm}$ and composed of 4 half-disks. Each half-disk made of 14648 trapezoidal crystals (7324 each) with a frontal area of $28.6 \times 28.6 \mathrm{~mm} 2$, and a length of $220 \mathrm{~mm}\left(24.7 X_{0}\right)$. The crystals in each disk are organized into 138 standard $5 \times 5$ supercrystal units with $52 \mathrm{~mm}$ void in between the groups. The crystals are arranged in a quasi-projective geometry pointing $\pm 1300 \mathrm{~mm}$ beyond the nominal interaction point. Vacuum phototriodes (VPTs) are used as photodetectors in the endcaps. 
A preshower detector is a pair of sampling calorimeters designed to distinguish neutral pions from real photons and improves the position measurement of electrons and photons with high granularity. Each calorimeter consists of two planes of silicon sensors interleaved with a total of $3 X_{0}$ of lead and is located in front of the endcaps and covers $1.65<|\eta|<2.60$.

\subsubsection{CMS Hadron Calorimeter}

The CMS hadron calorimeter (HCAL) 61] is used to measure the directions and energies of quarks and gluons, as well as the direction of neutrinos measuring the energy and direction of particle jets and of the missing transverse energy. The HCAL is composed of three sub-detectors, HCAL Barrel (HB) covering the central region up to $|\eta|<1.3$, the HCAL endcaps (HE) covering $1.3<|\eta|<3.0$, and the HCAL forward detector (HF) covering the the region $3.0<|\eta|<5.2$. In order to have a larger amount of material needed to absorb the hadron shower than the available volume in the barrel region, an additional outer HCAL (HO) is placed outside of the solenoid, behind the magnet. A schematic drawing of the HCAL can be seen in Fig. 3.7 .

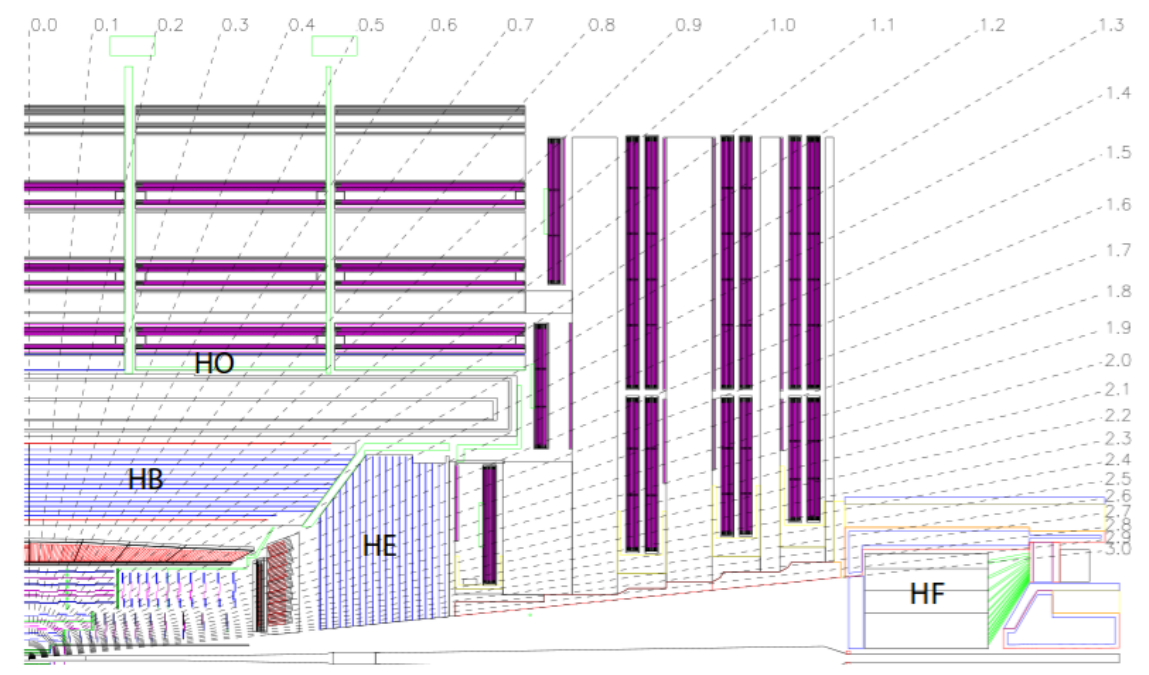

Figure 3.7: A schematic drawing of the CMS HCAL in the $r-z$ plane. The dashed lines denote different values of pseudorapidity. From Ref. [54].

Both the HB and HE are sampling calorimeters made out of copper alloy and stainless steel, and experience the 3.8 Tesla field of the CMS solenoid. The active material of plastic scintillators with a thickness of a few millimeters is placed between 5 (8) mm thick copper absorber plates. The barrel calorimeter has a sampling depth of $79 \mathrm{~cm}$. The innermost and outermost plates are $7 \mathrm{~cm}$ thick and are made of stainless steel for structural strength. The granularity of the HB and HE calorimeters 
depending on the $\eta$ coverage are $\Delta \eta \times \Delta \phi=0.087 \times 0.087$ for for $|\eta|<1.6$ and $\Delta \eta \times \Delta \phi=0.17 \times 0.15$ for $|\eta| \geq 1.6$.

To cope with the exceptionally high radiation dose (up to about $10 \mathrm{mSv} / \mathrm{h}$ ), the HF calorimeters use more robust, minimal-maintenance quartz fibers as an active material and steel as the absorber material. They have a diameter of $2.6 \mathrm{~m}$, cover the pseudorapidity range $3.0<|\eta|<5.0$, and are placed at $\pm 11.2 \mathrm{~m}$ away from the interaction point. Due to the increased coverage of the HF the instrumental $\not_{T}$ due to jet mismeasurement is reduced by an order of magnitude in the $20-120 \mathrm{GeV}$ energy range over that derived from the HB and HE alone.

Lastly, the HO has 5 rings and extends the total depth of the calorimeter system to a minimum of $11.8 \lambda_{\mathrm{I}}$ and ensures adequate sampling depth for $|\eta|<1.3$. The central ring has two layers of HO scintillators of a $19.5 \mathrm{~cm}$ thick piece of iron at radial distances of $3.82 \mathrm{~m}$ and $4.07 \mathrm{~m}$. All other rings have a single $\mathrm{HO}$ layer at a radial distance of $4.07 \mathrm{~m}$. Scintillator tiles are made from Bicron BC408 scintillator plates and the scintillation light is read out by wavelength shifting fibers.

\subsubsection{CMS Muon System}

The CMS muon system is designed to measure the trajectory of muons over a large kinematic range. The long lifetime of the muon and the absorption of hadrons by the calorimeters leads to the positioning of the muon system as the outermost subdetector providing excellent identification capability. The muon system has a hermetic coverage over the pseudorapidity range up to \pm 2.4 . It consists of three different technologies to detect and measure the trajectory of muons. The drift tubes (DT) are in the barrel region and extent up to 1.3 in $|\eta|$, cathode strip chambers (CSC) are in the endcap region cover from 0.9 to 2.4 in $|\eta|$, and resistive-plate chambers (RPC) are in both the barrel and endcap and cover the region from 0 to 2.1 in $|\eta|$ and provide additional sensitivity to backgrounds. An overview of the muon system can be seen in Fig. 3.8

The barrel muon system is composed of four stations two of which are mounted on the inner and the outer faces of the return yoke of the magnet. The other two are positioned inside the steel yoke. The first three stations consist of 8 chambers each, and are used to measure the muon coordinate in the $r-\phi$ plane and provide a measurement in the z-direction.

The endcap muon system has four muon stations (ME1, ME2, ME3, ME4) of CSCs and can operate at high rates and in large magnetic field. The CSCs have trapezoidal shape, and are arranged to overlapping to provide continuous $\phi$ coverage. Each CSC consists of 6 anode wires interlayer between 7 cathode panels. The anode wires are placed radially to measure a track's radial 


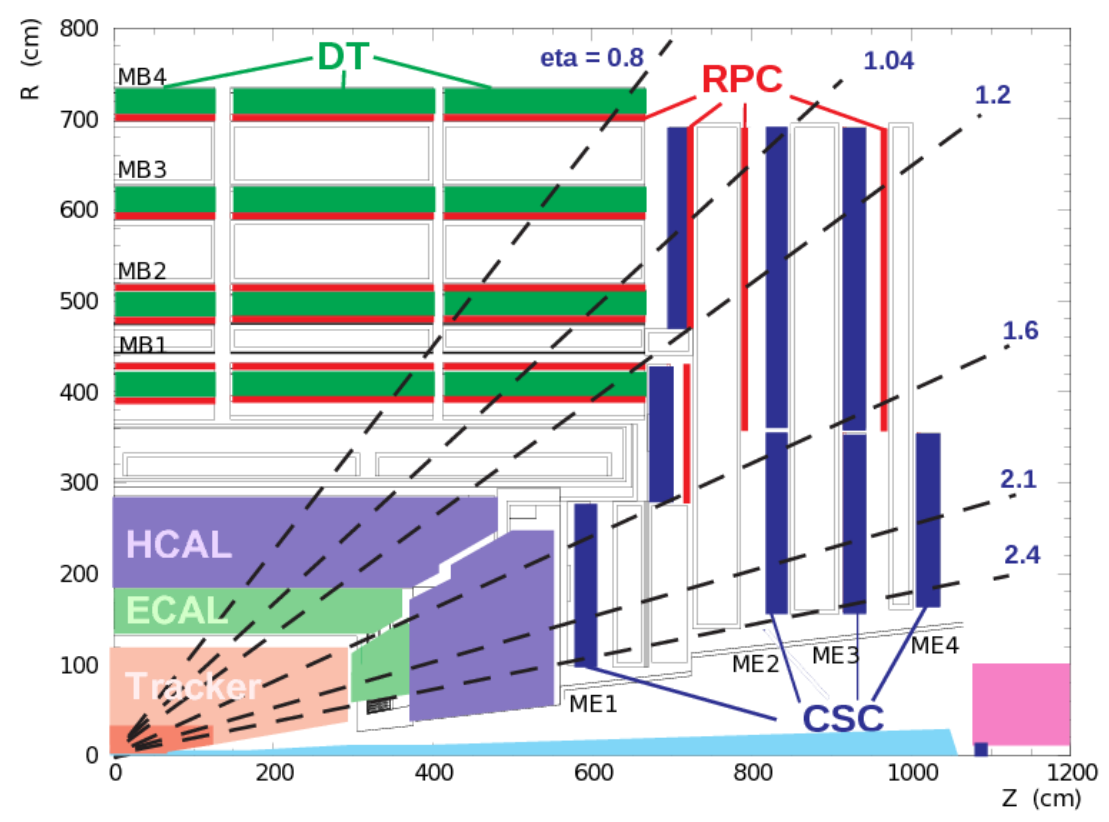

Figure 3.8: Quarter-view of the CMS detector with CSCs of the endcap muon system highlighted in red. From Ref. [54.

coordinate. The cathodes run lengthwise at a constant $\Delta \phi$ width.

The RPCs are placed both in barrel and endcaps. RPCs are gaseous parallel-plate chambers that provide good spatial resolution with excellent time resolution. They cover nearly the same area as DTs and CSCs but gives out a faster timing to identify signal and also have different sensitivity to the cosmic ray backgrounds.

\subsubsection{CMS Trigger and Data Acquisition Systems}

The CMS trigger select collisions of potential physics interest through a two-layer system 62 . The first level of the trigger (L1) is a hardware system designed to select events that contain (a combination of) candidate objects, such as ionization deposits consistent with a muon, clusters consistent with a electron, photon, or tau, missing transverse energy $\left(\not{ }_{T}\right)$ or a jet, within $4 \mu \mathrm{s}$ of a collision. The final selection at this level is based on 128 programmable algorithms with adjustable threshold on the output rate up to $100 \mathrm{kHz}$. The selected events are then passed to the second level, High-Level Trigger (HLT). The HLT is software based, and is used to select events with higher purity of the physics objects for offline storage. Depending on the LHC instantaneous luminosity the HLT output rate can vary between $300 \mathrm{~Hz}-700 \mathrm{~Hz}$. To reduce the output rate both at the L1 and HLT, algorithms can be prescaled to accept only a fraction of events, which passed the selection 
criteria defined by a specific algorithm. The HLT is part of the Data Acquisition System (DAQ), which manages the overall flow of data. The DAQ also consists of detector front-end electronics, readout modules, an event builder network, as well as management and monitoring systems.

\subsubsection{Level 1}

A schematic overview of the L1 trigger can be seen in Fig. 3.9. Information from the trigger primitives (TP) acquired from the ECAL and HCAL, as well as from the muon detectors: drift tubes (DT), cathode strip chambers (CSC), and resistive plate chambers (RPC) are handled in Global Calorimeter Trigger (GCT) and the Global Muon Trigger (GMT), respectively. The combined information from these two systems is forwarded to the Global Trigger (GT) for the final decision to accept the event.

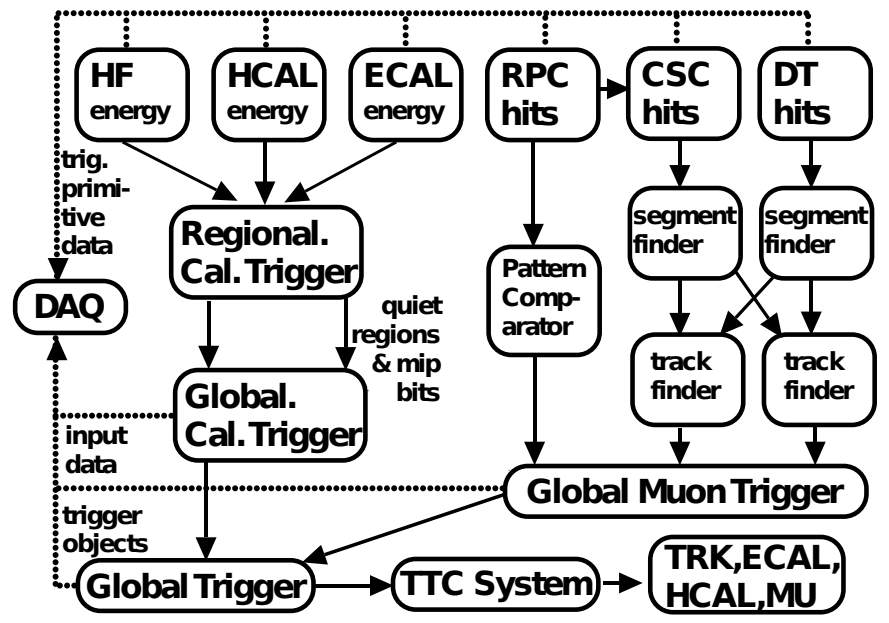

Figure 3.9: Overview of the CMS L1 trigger.

The L1 calorimeter trigger consists of two systems, a regional calorimeter trigger (RCT) and global calorimeter trigger $(\mathrm{GCT})$. The RCT collects information about the regional $E_{\mathrm{T}}$ sums $(4 \times 4$ towers) from ECAL and HCAL towers that cover up to $|\eta|<5$. It sends this output along with the e $/ \gamma$ candidates to GCT. In the GCT the e $/ \gamma$ candidates are further sorted, jets are identified in 3 categories (central, forward, and tau) and global quantities such as total and missing $E_{\mathrm{T}}$ are calculated 6366 .

L1 muon trigger uses information from the DT, CSC, and RPC detectors. The DT and the CSC systems are used to identify track segments from the hit information registered in a single measurement station. The segments are then transferred to DT track-finder (DTTF) and CSC 
track-finder (CSCTF) where muon candidates are identified and their momenta are determined through pattern recognition algorithms. On the other hand, the hits from the RPC are sent to the pattern comparator logic boards (PAC) to identify muon candidates. The identified muon candidates along with their assigned $p_{\mathrm{T}}$ and quality code, including the $(\eta, \phi)$ position in the muon system, are transferred to the global muon trigger (GMT) where further quality requirements are imposed. If the muon candidate is reconstructed only by one muon track-finder or fails the additional quality cuts, the candidate is discarded.

The Global Trigger (GT) is the final step of the CMS L1 Trigger and is responsible for applying the final set of selection requirements to the candidate objects coming from the muon and the calorimeter systems. The GT also synchronizes data arriving at different times and propagates the L1 decision to the timing, trigger, and control (TTC) system.

In conclusion the L1 trigger menu will include maximum of 128 separate selections based on simple single objects with varied $E_{\mathrm{T}}$ thresholds and/or in case of several objects with topological conditions.

\subsubsection{High-Level Trigger}

The events that pass the L1 trigger algorithms are passed on to the HLT where objects are reconstructed closer to the offline fashion with further identification criteria applied to select events that are interesting for offline data analysis.

The hardware part of the HLT is composed of the event filter farm (EVF) of CPUs with filter and builder units. In total, the EVF consisted of $13000 \mathrm{CPU}$ cores at the end of 2012. Builder units are responsible for building complete events using the information coming from the individual detectors. Filter units use the built event coming from the builder units to unpack the raw data into detector-specific data structures and to perform the event reconstruction and trigger filtering. The filter farm uses significant CPU resources to handle the high input rate of $100 \mathrm{kHz}$ with an average processing time of up to $\sim 175 \mathrm{~ms}$ per event. The HLT design specification are described in detail in Ref. 67].

Data processing at HLT utilizes sophisticated offline-quality reconstruction algorithms with an optimized performance to minimize the dead-time. These algorithms are processed in a predefined order in an HLT path that reconstructs and make selections on these objects. The algorithms in each HLT paths are executed in the order of increasing complexity to reduce the input rate before the CPU-expensive reconstruction, such as the particle-flow algorithm.

After the full online reconstruction and filtering the final decision to keep or reject the event is 
made, events that are accepted are sent to the storage manager where the event data is stored locally on disk until transferred to the CMS Tier-0 center at CERN for offline processing and permanent storage. The output rate of the HLT is limited by the event size and ability for the CERN Tier-0 to process the events. Most events except that are part of the "parked" data program are processed instantaneously. With the data parking program, CMS recorded additional data with relaxed trigger requirements planning for a delayed offline reconstruction in 2013 after the completion of the LHC Run I.

A convenient measure to quantify the rate of the the HLT menu is the "cross section". It is defined as the event rate divided by the instantaneous luminosity. A plot of the HLT cross section during 2012 can be seen in Fig. 3.10 .

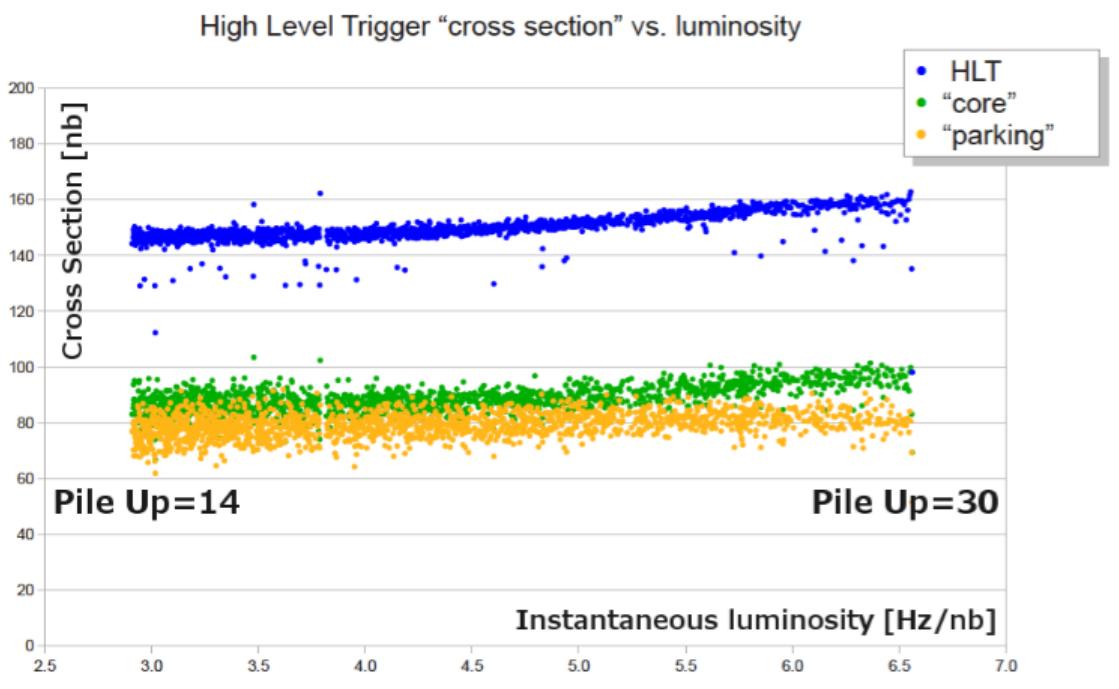

Figure 3.10: Cross section of the HLT menu in 2012 as a function of the instantaneous luminosity. From Ref. 68.

\subsubsection{DAQ}

The DAQ system assembles data fragments from separate detector components into full events in two stages and transports them between the L1, HLT, and data storage center. The DAQ system sustains an input rate of $100 \mathrm{kHz}$, for a data flow of $\sim 100$ GByte/s. It also provides enough computing power for the HLT to reduce the rate of stored events by a factor of 1000. The architecture of the CMS Data Acquisition (DAQ) system is shown schematically in Fig. 3.11 .

The first stage begins when an event is accepted by the L1, after which data fragments corresponding to the accepted event are read out from the front-end drivers (FEDs). The event builder 


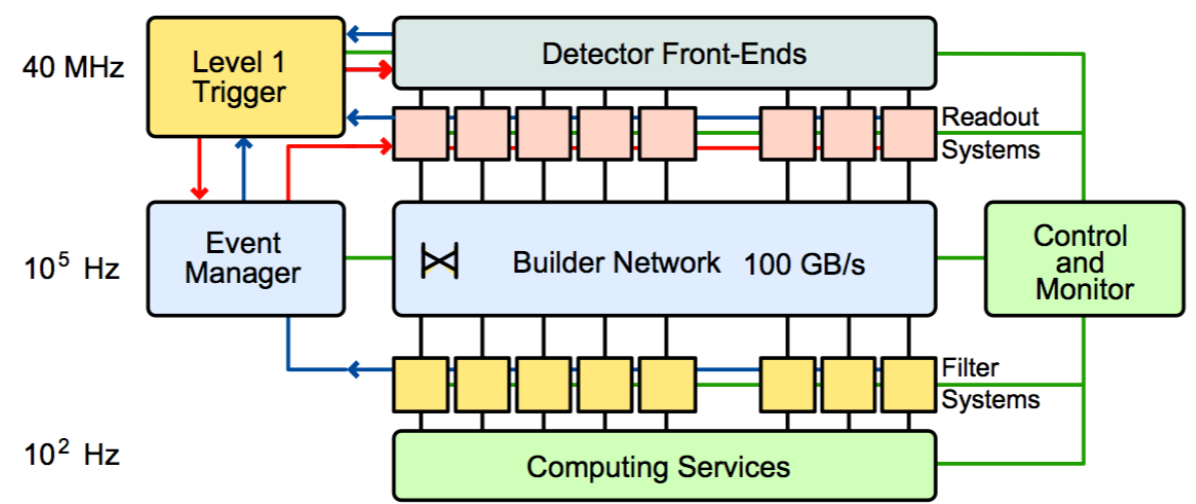

Figure 3.11: Simplified sketch of the CMS DAQ system. From Ref. 69.

assembles the event fragments belonging to the same L1 decision from all FEDs into a complete event and transmits it to a Filter Unit (FU) for further processing.

The DAQ system utilizes up to 8 slices that work autonomously and can each handle $12.5 \mathrm{kHz}$ event rate. The system can experience back-pressure all the way from the filter farm through the event builder to the FEDs. Back-pressure may occur when the buffer overflows in the sub-detector's FEDs due to the variations in the size or rate of events and could lead to data corruption and loss of synchronization. The Trigger-Throttling System (TTS) is designed to protect the system against these buffer overflows. TTS system gives a fast feedback from any sub-detector FEDs to the GT processor so that the trigger rate could be controlled before the buffers overflow. Furthermore, both the L1 trigger and HLT prescales could be adjusted to optimize the available DAQ capacity and performance during operation. However, instantaneous fluctuations might still lead to back-pressure and therefore introduce dead-time.

The collected events are divided into different physics or calibration streams and forwarded to the Tier-0 centre for offline processing. Different reconstruction algorithms are employed for different streams. Events that are in the physics stream will undergo a reconstruction algorithm using the full detector information, whereas the calibration and monitoring streams will undergo a reconstruction using only a subset of the sub-detector information. 


\section{Chapter 4}

\section{Event and Object Reconstruction}

The event and object related reconstruction techniques with the CMS detector relevant for this analysis are discussed in this chapter.

The CMS experiment utilizes a Particle Flow (PF) algorithm [70] designed to uniquely identify all stable particles from each pp collision event by combining information from all CMS sub-detectors. The PF approach is possible in CMS due to the excellent angular and energy resolution of the CMS silicon tracker and electromagnetic calorimeter. The particle flow algorithm makes use of an iterative tracking algorithm designed to maintain high efficiency in the dense environment typical for jets, as well as a low probability of misreconstructed tracks. The tracking misreconstruction rate is less than a per cent even for the most difficult scenarios, such as low $p_{\mathrm{T}}(\sim 100 \mathrm{MeV} / c \%)$ tracks originating far from the beam axis. For calorimeter deposits, a specific clustering algorithm is used which is optimized to maintain high efficiency for low-energy particles and to effectively separate nearby deposits. A "linking" algorithm is then used to associate tracks with calorimetric deposits. The PF algorithm identifies muons by comparing silicon tracks to tracks reconstructed by the CMS muon system, and electrons are reconstructed and identified using tracking and calorimetric variables. The remaining particles can be identified as charged hadrons, neutral hadrons, or photons by comparing the momentum of tracks with the calorimeter deposits. The final list of PF particles can be used to apply more stringent identification cuts, as input to a jet algorithm, or to compute missing energy.

\subsection{Trigger Design and Implementation}

The CMS Level-1 GCT produces electron and photon $(\mathrm{e} / \gamma)$ candidates for triggering. The L1 e/ $\gamma$ trigger algorithm utilizes a sliding window of $3 \times 3$ trigger towers (TTs). The transverse energy of 
the $\mathrm{e} / \gamma$ candidate is calculated from the energy deposit of the central tower summed with the largest deposit in one of its 4 neighbor towers, as shown in Fig. 4.1 a. Only candidates in which two adjacent strips ( 5 crystals in $\phi$ are called a strip) of the central tower contain $90 \%$ of the tower energy are kept. The associated HCAL energy contribution is required to be less than $5 \%$ of the ECAL energy. To qualify as an isolated candidate, at least 5 of the 8 adjacent TTs must have transverse energy below a certain threshold. The GCT can produce four isolated and four non-isolated e $/ \gamma$ candidate objects per event for trigger decisions. At L1, only information from the calorimeter is available and no distinction can be made between electrons and photons.

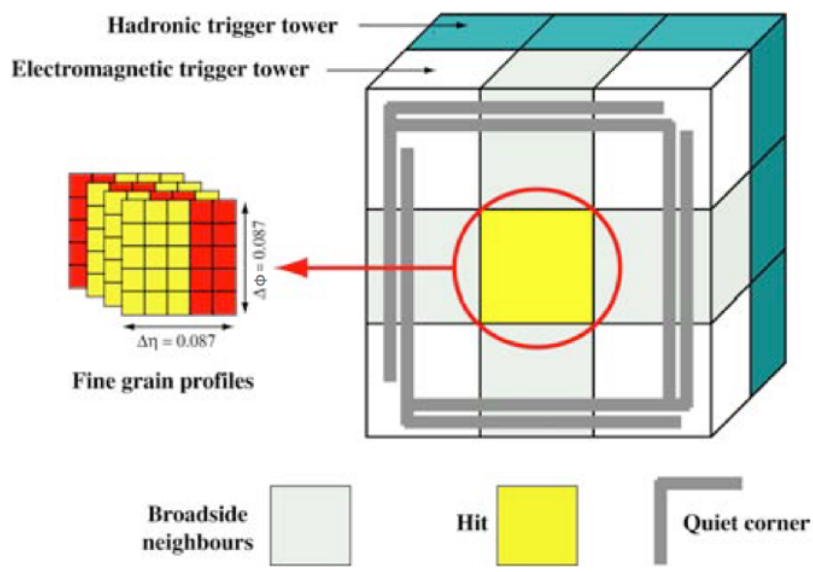

Figure 4.1: Pictorial representation of the transverse energy calculation of an e/ $\gamma$ deposition.

To evaluate the performance of the $\mathrm{L} 1 \mathrm{e} / \gamma$ trigger, electrons reconstructed offline are matched to $\mathrm{L} 1 \mathrm{e} / \gamma$ candidates by looking for the $\mathrm{RCT}$ region that contains the highest energy $\mathrm{TT}$ within the electron "supercluster", which is a cluster of clusters. The resolution is extracted by comparing the transverse energies of the offline reconstructed supercluster and the corresponding L1 candidate. Fig. 4.2 shows the distribution of the $\mathrm{L} 1 \mathrm{e} / \gamma$ trigger resolution separately for the ECAL barrel and endcap. The resolution of $\mathrm{L} 1 \mathrm{e} / \gamma$ candidates shown in Fig. 4.2 is approximated by a CrystalBall function 71]. The energy of an electron supercluster can be spread over a large region of the calorimeter due to the emission of photons via bremsstrahlung.

The efficiency of the $\mathrm{L} 1 \mathrm{e} / \gamma$ trigger was measured with electrons from $Z \rightarrow e e$ events using a "tag-and-probe" method 72 using the full dataset collected during 2011 and 2012. The tag electron is required to trigger the event at L1, while the probe electron is used for the efficiency studies. Both the tag electron and the probe electron are required to pass tight identification criteria to reduce the background contamination. The invariant mass of the tag-and-probe system is required to be consistent with the $Z$ boson mass $\left(60 \mathrm{GeV}<m_{e e}<120 \mathrm{GeV}\right)$. These requirements result in a pure 

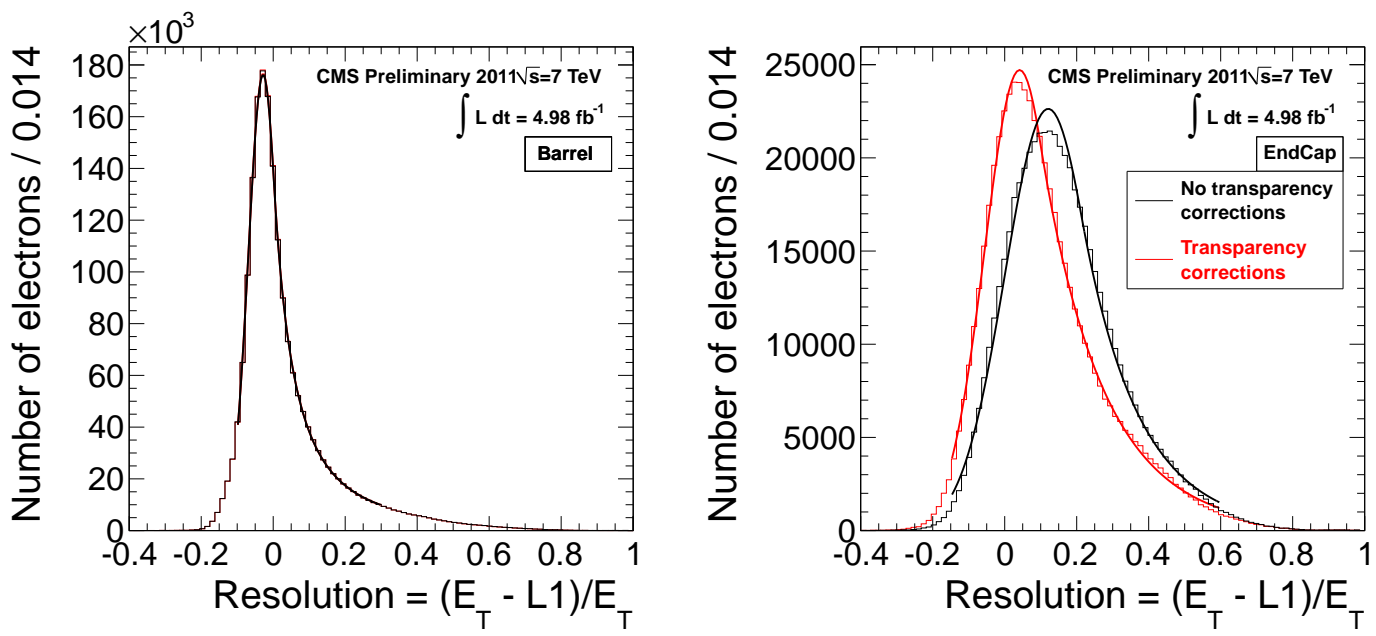

Figure 4.2: $\mathrm{L} 1 \mathrm{e} / \gamma p_{\mathrm{T}}$ resolution for electrons located in the Barrel (left) and in the Endcaps (right). For both distributions, a Crystal-Ball fit is superimposed. In the right pane, the red solid line shows the result after applying the transparency corrections (see text).

and unbiased electron data sample. The trigger efficiency is given by the fraction of probes above a given transverse energy $\left(E_{\mathrm{T}}\right)$ threshold, as a function of the probe $E_{\mathrm{T}}$. Fig 4.3 shows the performance of the single e $/ \gamma$ trigger algorithm with an $E_{\mathrm{T}}$ threshold of $20 \mathrm{GeV}$ during the 2012 data-taking period. The widths of the turn-on curves are a consequence of the coarse trigger granularity, which degrades the energy resolution. In the ECAL endcap region, the larger amount of material in front of the calorimeter increases the probability of bremstrahlung resulting in a slower turn-on curve.

The HLT algorithms for the e $/ \gamma$ trigger selection begin by combining energy deposits around an L1 candidate to form a supercluster (SC) using the same algorithms as in the offline reconstruction. The clustering algorithm accounts for the spread of bremsstrahlung energy in the $\phi$ direction due to the magnetic field. At the HLT, the full granularity of the ECAL and HCAL subdetectors is used to form e $/ \gamma$ candidates and apply selection requirements such as cluster shape and isolation variables. Electrons can be distinguished from photons by requiring the presence of a track compatible with the SC. The energy and position of the SC are used to propagate a trajectory through the magnetic field to check for compatible hits in the pixel detector. The reconstruction of the electron tracks can be improved by the Gaussian Sum Filtering (GSF) algorithm, which parametrizes the highly nonGaussian loss of energy of the electron track. The trigger rate of the lowest threshold single-electron path $\left(E_{\mathrm{T}}\right.$ threshold of $\left.27 \mathrm{GeV}\right)$ at different stages of the trigger selection is shown in Fig. 4.4 .

The lead tungstate crystals of the CMS ECAL begin to lose their transparency when they undergo irradiation, and the transparency is partially recovered during breaks in data-taking. Therefore 


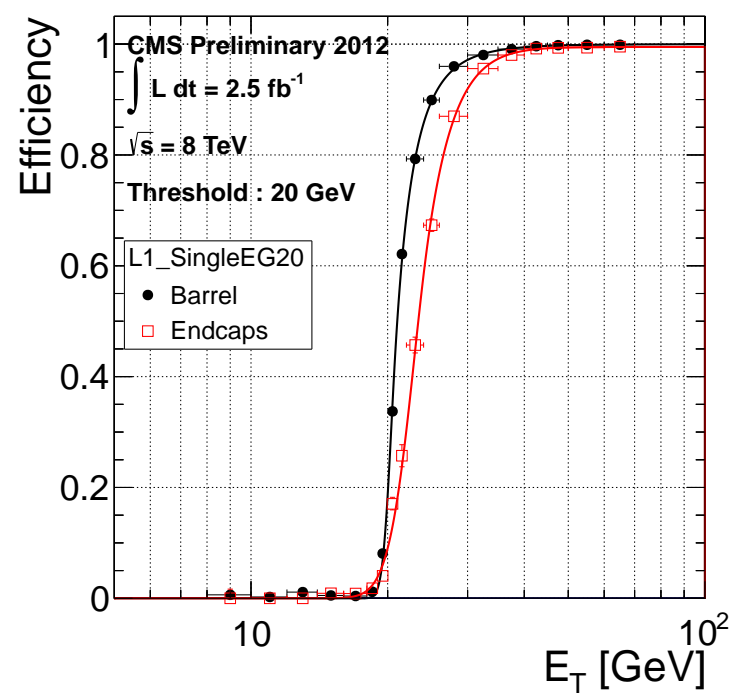

Figure 4.3: Electron trigger efficiency at L1 ("EG" threshold : $20 \mathrm{GeV} E_{\mathrm{T}}$ ), as a function of $E_{\mathrm{T}}$ for electrons in the EB (black dots) and EE (red dots), using the 2012 dataset.

the response of the ECAL varies throughout the data-taking period. A laser system monitors the transparency of each crystal, allowing the measured energies to be corrected. The effect of irradiation on the efficiency turn-on curves of e/ $\gamma$ objects can be seen in Fig. $4.4 \mathrm{~b}$.
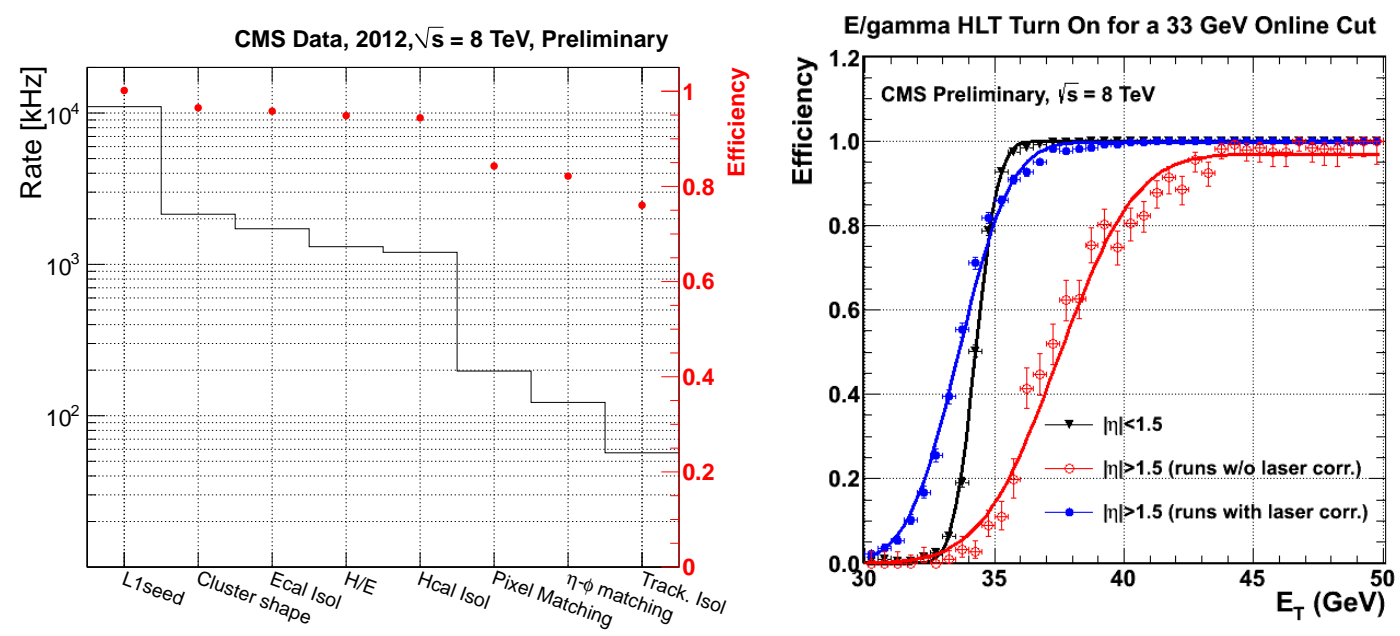

Figure 4.4: The left plot shows how the rate is gradually reduced by the filtering steps of this trigger (black) along with the efficiency on electrons (red). The right plot shows the electron efficiency as a function of offline $E_{T}$ in the EB and EE before and after the transparency corrections at HLT

The triggers used for the analysis of the $\gamma+\not \phi_{T}$ final state are listed in Table 4.1. The main signal was implemented during the CMS data parking program and recorded a data set corresponding to an integrated luminosity of $7.3 \mathrm{fb}^{-1}$. The trigger is seeded by events passing a single $e / \gamma \mathrm{L} 1$ trigger with an $E_{\mathrm{T}}$ threshold of $20 \mathrm{GeV}$. The HLT selection requires the presence of a $30 \mathrm{GeV}$ isolated 
photon within $|\eta|<1.4442$ and calorimetric $\not_{T}>25 \mathrm{GeV}$. The trigger has further the following photon identification requirements (which will be explained in detail in the following section):

- $f_{h}<0.10$

- $\sigma_{i \eta i \eta}<0.024$

- $R_{9}>0.9$

- ECAL Isolation $<4.0+0.012 \times E_{T}$;

- HCAL Isolation $<4.0+0.005 \times E_{T}$;

- Track Isolation $<4.0+0.002 \times E_{T}$.

Table 4.1: Triggers used for the main analysis and for the efficiency measurement.

\begin{tabular}{|l|}
\hline Signal Trigger \\
\hline HLT_Photon30_R9Id90_CaloID_HE10_Iso40_EBOnly_Met25_HBHENoiseCleaned \\
\hline \hline Efficiency Measurement / Monitoring Triggers \\
\hline HLT_Photon30 (prescaled by 500) \\
HLT_Photon30_R9Id90_CaloID_HE10_Iso40_EBOnly (prescaled by 20) \\
\hline
\end{tabular}

The efficiency of the Level-1 $e / \gamma$ trigger has been measured is shown in Fig. 4.3. Two prescaled control triggers, also shown in Table 4.1, are used to measure separately the HLT efficiency of the photon and $\not_{T}$ portions of main signal trigger. The first control trigger is a $30 \mathrm{GeV}$ photon trigger with no isolation or identification selection. This trigger is used to measure the efficiency of the photon isolation and identification requirements in the main signal trigger by considering the ratio:

$$
\frac{\text { passed (HLT_Photon30 \&\& HLT_Photon30_Id \&\& Offline Photon Selection) }}{\text { passed (HLT_Photon30 \&\& Offline Photon Selection) }} .
$$

The second control trigger is a copy of the main signal trigger but without any $\not_{T}$ selection, and is used to measure the efficiency of the HLT $\not_{T}$ selection by considering the ratio:

$$
\frac{\text { passed (HLT_Photon30_Id \&\& HLT_Photon30_Id_Met \&\& Offline Photon Selection) }}{\text { passed (HLT_Photon30_Id \&\& Offline Photon Selection) }} .
$$

The efficiencies are measured as a function of the $p_{\mathrm{T}}$ of offline reconstructed and identified photons (matched to the triggering photon) and $\not \phi_{T}$. The results of the HLT efficiencies for the photon and 
$\not_{T}$ portions of the trigger are shown in Fig. 4.5, as a function of the photon $p_{\mathrm{T}}$ and particle-flow $\not_{T}$, respectively. The efficiencies are parametrized using an error function. The fit function used is:

$$
\frac{p_{2}}{2} \cdot\left(1+\operatorname{Err}\left(\frac{x-p_{0}}{p_{1} \cdot \sqrt{2}}\right)\right)
$$

and the parameters are given in Table 4.2. We used a histogram with variable bin size to ensure that the fitted values represent the behavior in all energy ranges. We have varied the central values of the fits within their uncertainties to estimate the uncertainty due to the trigger efficiency measurement, and it was found to be less than $0.05 \%$.

Table 4.2: The fit parameters for the trigger efficiency curves

\begin{tabular}{|c|c|c|}
\hline Parameter & Photon ID Efficiency & $\not_{T}$ Efficiency \\
\hline \hline$p_{0}$ & $18.83 \pm 7.5$ & $27.67 \pm 0.11$ \\
$p_{1}$ & $7.52 \pm 4.4$ & $25.17 \pm 0.32$ \\
$p_{2}$ & $0.89 \pm 0.005$ & $0.97 \pm 0.003$ \\
\hline
\end{tabular}
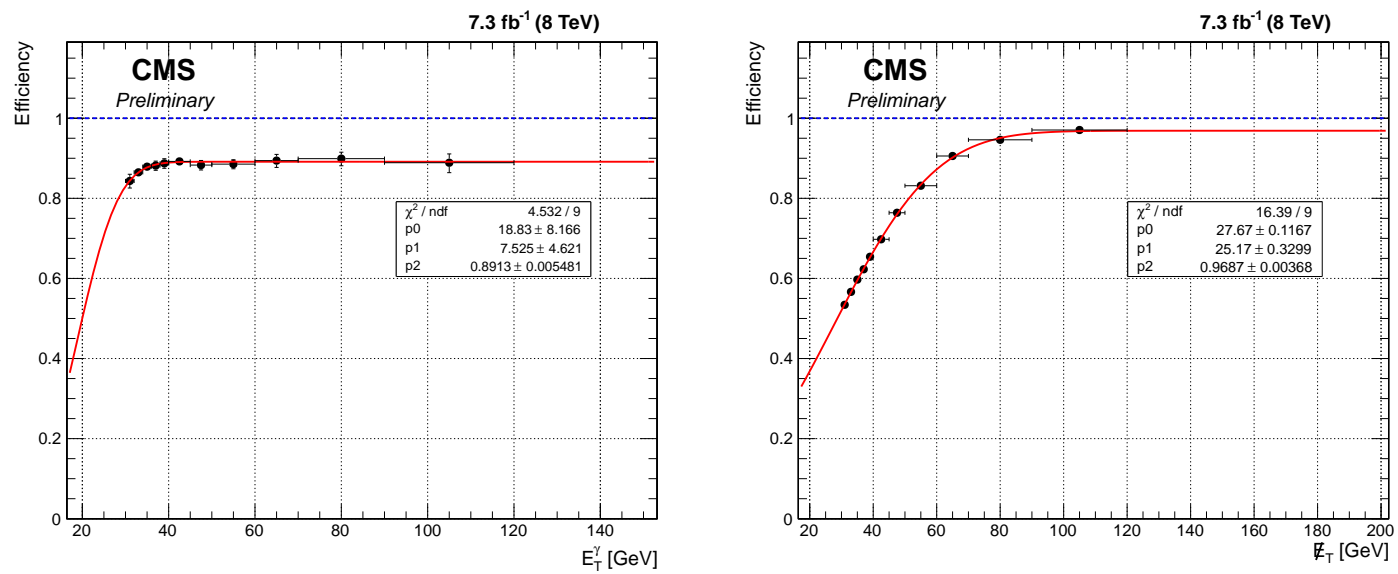

Figure 4.5: Efficiency of the HLT photon selection as a function of the offline reconstructed photon $p_{\mathrm{T}}$ (left) and efficiency of the HLT $\not_{T}$ selection as a function of the offline reconstructed particle-flow $\not_{T}$.

\subsection{Photon Reconstruction and Identification}

Photons are reconstructed from energy deposits in the ECAL using clustering algorithms generally limited to a fiducial region excluding the last two crystals at each end of the barrel $(|\eta|<1.4442)$. The clustering algorithms evolve from fixed matrices of $5 \times 5$ crystals to give the best reconstruction of unconverted photons and do not constrain the particle to be originating from the interaction point. 
Clusters are built starting from the crystal containing the signal corresponding to a transverse energy larger than in its immediate neighbors. Such crystals are also called the "seed crystals". In the barrel, the clusters have a fixed width of five crystals centered around the seed crystal in the $\eta$ direction. In the $\phi$ direction, adjacent strips of five crystals are added.

The energy resolution for a single photon is measured using $Z \rightarrow \mathrm{e}^{+} \mathrm{e}^{-}$events in both data and simulated events where the electron showers are reconstructed as photons. Fig. 4.6 shows the energy resolution of a single photon measured as the position of the shower in the ECAL for two different regions of $R_{9}$. The $R_{9}$ variable is defined as the energy sum of the $3 \times 3$ crystals centered on the seed crystal divided by the energy of the supercluster. The showers of photons that convert before reaching the calorimeter have wider spread in their cluster shape and lower values of $R_{9}$ with a degraded photon energy resolution than those of unconverted photons. Figure 4.7 shows the $R_{9}$ distribution for photons in the ECAL barrel that convert in the material of the tracker, and those that convert later, or do not convert at all before reaching the ECAL. The simulated resolution matches the resolution observed in data as a function of $\eta$ well.

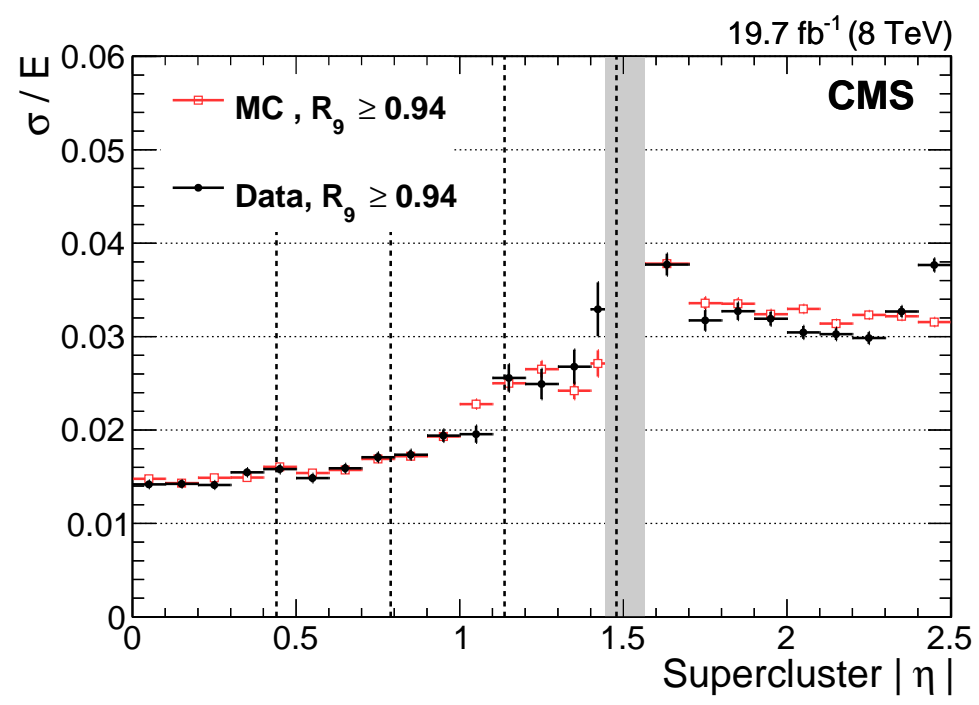

Figure 4.6: Fractional photon energy resolution measured in bins of absolute supercluster pseudorapidity in $Z \rightarrow \mathrm{e}^{+} \mathrm{e}^{-}$events, for data (black points) and simulated events (red points), where the electrons are reconstructed as photons. From Ref. 73

The photon identification prescription used in this analysis is the "pixel track seed veto" to reject electrons. This veto rejects any photon for which a pixel track seed consisting of at least two hits in the pixel detectors is found. The fractions of photons and electrons passing the pixel track seed veto is given in Table 4.3

Further photon identification variables are split into two main categories of observables: shower 


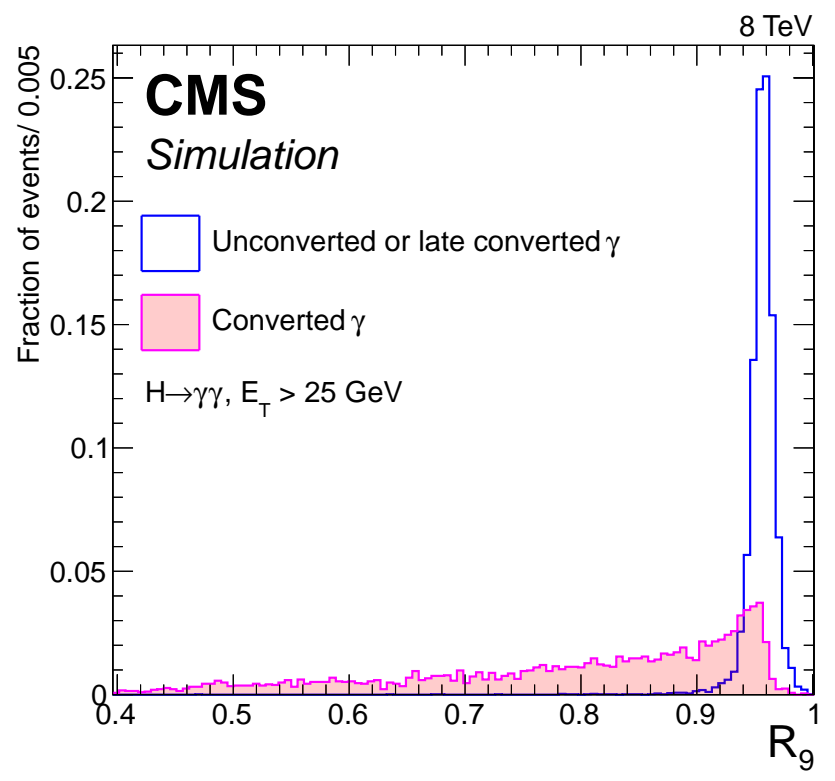

Figure 4.7: Distributions in the $R_{9}$ variable for photons in the ECAL barrel that convert in the material of the tracker (solid filled histogram), and those that convert later, or do not convert at all before reaching the ECAL (outlined histogram). From Ref. 73

Table 4.3: Fractions of photons and electrons, in the ECAL barrel and endcap, passing the pixel track seed veto. The statistical uncertainties in the values given for electrons are negligible.

\begin{tabular}{|c|cc|cc|}
\hline & \multicolumn{2}{|c|}{ Barrel } & \multicolumn{2}{c|}{ Endcap } \\
& $\gamma$ & $\mathrm{e}$ & $\gamma$ & $\mathrm{e}$ \\
\hline Pixel track seed veto & $94.4 \pm 0.2 \%$ & $1.4 \%$ & $81.0 \pm 0.6 \%$ & $4.3 \%$ \\
\hline
\end{tabular}

shape and isolation variables. The variable $\sigma_{i \eta i \eta}$ is the lateral extension of the shower, and is measured in terms of the energy weighted spread within the $5 \times 5$ crystal matrix centered on the seed crystal 74. Photon candidates are further required to satisfy $f_{h}>0.05$, where $f_{h}$ is the ratio between the energy collected by the HCAL towers behind the supercluster and the energy of the ECAL supercluster.

Particle candidates that are used to compute the isolation sums are considered within a circular region, radius $\Delta R$, in the $(\eta, \phi)$ plane around the photon direction. The photon isolation variables are then obtained by summing the transverse momenta of charged hadrons, $I_{\pi}$, photons, $I_{\gamma}$, and neutral hadrons, $I_{\mathrm{n}}$, inside the isolation region. However, when calculating the $I_{\gamma}$, photons falling in a slice of size $\Delta \eta=0.015$ are excluded from the sum. Similarly, when constructing $I_{\pi}$, a region of $\Delta R=0.02$ is excluded when summing the transverse momenta of charged hadrons.

The extra contribution coming from the overlapping proton-proton interactions (pileup) in the 
isolation region is estimated as $\rho \times A_{\text {eff }}$, where $\rho$ is the median of the transverse energy density per unit area in the event [75] and $A_{\text {eff }}$ is the area of the isolation region weighted by a factor that takes into account the dependence of the pileup transverse energy density on pseudorapidity. To reduce the dependence of the isolation variables on the number of pileup events, the extra contribution, calculated using $\rho$, is subtracted from the photon and neutral hadron sums (Fig. 4.8).
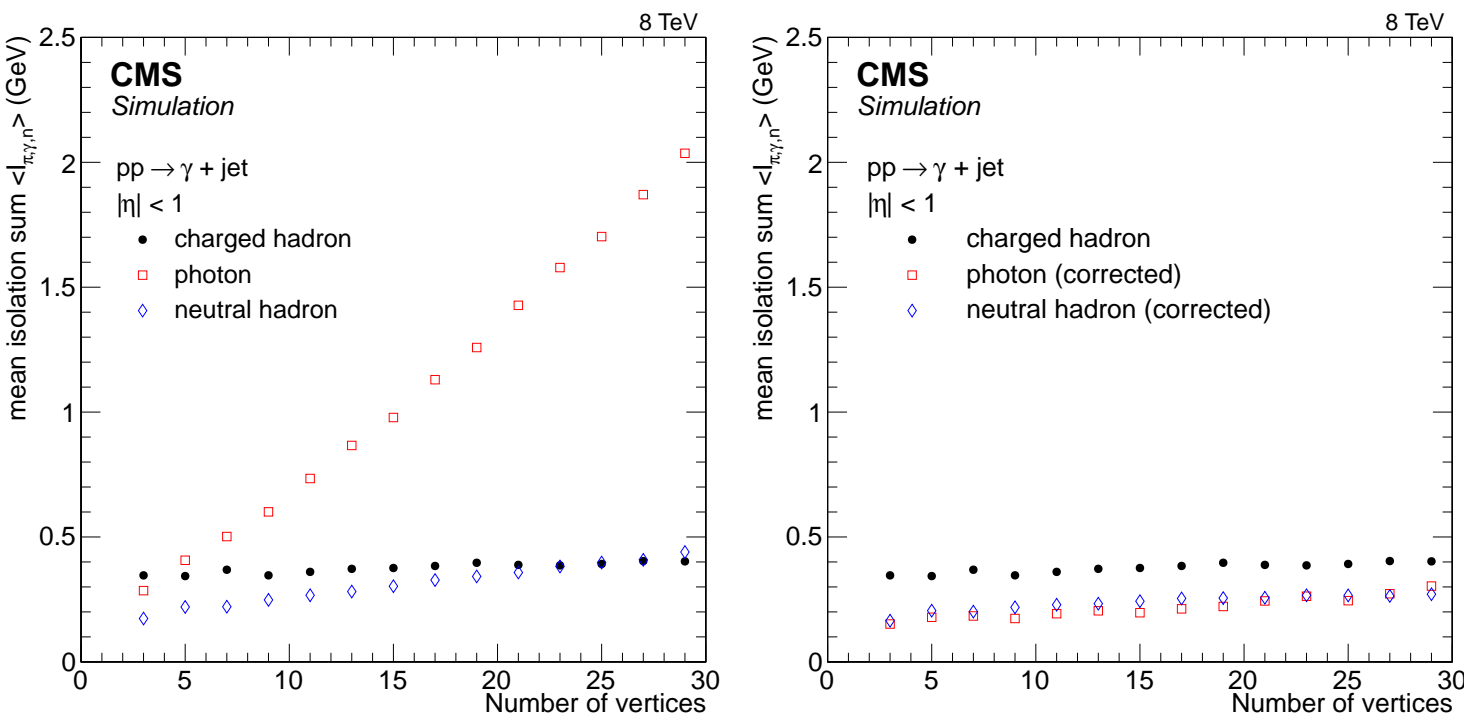

Figure 4.8: Mean value of the isolation variables for photons with $p_{\mathrm{T}}>50 \mathrm{GeV}$ in $\gamma+$ jet events, as a function of the number of reconstructed primary vertices, for events (left) before and (right) after being corrected for pileup using the $\rho$ variable.

A summary of the photon identification requirements, are shown for three different working points in Table 4.4

For this analysis, the medium working point has been chosen, additional requirement on the $R_{9}$ variable is applied to match the trigger requirements. Photon identification efficiencies are measured with the "tag-and-probe" method, as described in Ref. [72, using samples of $Z \rightarrow \mathrm{e}^{+} \mathrm{e}^{-}$events. The results of these measurements are then used to correct the simulation for any mismodelling by evaluating the ratio of efficiencies in data and simulation.

\subsection{Missing Transverse Energy}

The missing transverse energy is defined as the negative vector sum of all visible particles, $\vec{H}_{\mathrm{T}} \equiv$ $-\sum \vec{p}_{\mathrm{T}}$. Momentum conservation dictates that $\overrightarrow{\mathscr{E}}_{\mathrm{T}}$ is equal, in the limit of a perfect detector efficiency and resolution, to the vector sum of transverse momentum of all undetected particles such as neutrinos or weakly interacting BSM particles. To determine the $\vec{E}_{\mathrm{T}}$ visible particles 
Table 4.4: Photon identification requirements for three working points, corresponding to selections of different stringency.

\begin{tabular}{|c|c|c|c|}
\hline & Loose & Medium & Tight \\
\hline $\begin{array}{r}\text { barrel } \\
\text { endcap }\end{array}$ & $\begin{array}{c}1.3 G e V+0.005 E_{\mathrm{T}}^{\gamma} \\
-\end{array}$ & $\begin{array}{r}0.7 G e V+0.005 E_{\mathrm{T}}^{\gamma} \\
1 G e V+0.005 E_{\mathrm{T}}^{\gamma}\end{array}$ & $\begin{array}{r}0.7 G e V+0.005 E_{\mathrm{T}}^{\gamma} \\
1 G e V+0.005 E_{\mathrm{T}}^{\gamma}\end{array}$ \\
\hline $\begin{array}{r}\text { barrel } \\
\text { endcap }\end{array}$ & $\begin{array}{l}3.5 \mathrm{GeV}+0.04 E_{\mathrm{T}}^{\gamma} \\
2.9 \mathrm{GeV}+0.04 E_{\mathrm{T}}^{\gamma}\end{array}$ & $\begin{array}{l}1.0 \mathrm{GeV}+0.04 E_{\mathrm{T}}^{\gamma} \\
1.5 \mathrm{GeV}+0.04 E_{\mathrm{T}}^{\gamma}\end{array}$ & $\begin{array}{l}0.4 \mathrm{GeV}+0.04 E_{\mathrm{T}}^{\gamma} \\
1.5 \mathrm{GeV}+0.04 E_{\mathrm{T}}^{\gamma}\end{array}$ \\
\hline $\begin{array}{r}\text { barrel } \\
\text { endcap }\end{array}$ & $\begin{array}{l}2.6 \mathrm{GeV} \\
2.3 \mathrm{GeV}\end{array}$ & $\begin{array}{l}1.5 \mathrm{GeV} \\
1.2 \mathrm{GeV}\end{array}$ & $\begin{array}{l}0.7 \mathrm{GeV} \\
0.5 \mathrm{GeV}\end{array}$ \\
\hline $\begin{array}{lr}\sigma_{\text {inin }} & \text { barrel } \\
& \text { endcap }\end{array}$ & $\begin{array}{l}0.012 \\
0.034\end{array}$ & $\begin{array}{l}0.011 \\
0.033\end{array}$ & $\begin{array}{l}0.011 \\
0.031\end{array}$ \\
\hline $\begin{array}{l}f_{h} \\
\text { Electron veto }\end{array}$ & & $\begin{array}{c}0.05 \\
\text { Pixel seed veto }\end{array}$ & \\
\hline
\end{tabular}

Table 4.5: Scale factors for the photon identification used for the analysis

\begin{tabular}{|c|c|c|c|}
\hline Variable & $E_{\mathrm{T}}^{\gamma}[30-40] \mathrm{GeV}$ & $E_{\mathrm{T}}^{\gamma}[40-50] \mathrm{GeV}$ & $E_{\mathrm{T}}^{\gamma}>50 \mathrm{GeV}$ \\
\hline \hline Pixel Seed Veto & $0.995 \pm 0.004$ & $0.970 \pm 0.016$ & $1.012 \pm 0.014$ \\
Photon ID $(0<|\eta|<0.8)$ & $0.996 \pm 0.010$ & $0.996 \pm 0.010$ & $0.996 \pm 0.010$ \\
Photon ID $(0.8<|\eta|<1.5)$ & $0.996 \pm 0.010$ & $0.997 \pm 0.010$ & $0.997 \pm 0.010$ \\
\hline
\end{tabular}

are reconstructed using the CMS particle-flow (PF) technique. This type of missing transverse energy is referred to as $\mathrm{PF} \vec{H}_{\mathrm{T}}$. Similarly, the PF $\sum E_{\mathrm{T}}$ is the associated (positive) scalar sum of the transverse momenta of all PF particles. If the $\vec{H}_{\mathrm{T}}$ is reconstructed without the use of PF candidates, using only the calorimeter energy deposits, it is referred to as Calo $\vec{E}_{\mathrm{T}}$.

Due to a wide variety of effects including the nonlinear response, inefficiencies, and minimum energy thresholds of the various CMS subdetectors, the magnitude of the $\overrightarrow{\mathscr{E}}_{\mathrm{T}}$ can be underestimated or overestimated. Therefore, several corrections are applied to the $\overrightarrow{\mathscr{H}}_{\mathrm{T}}$ measurement. A large portion of the bias can be reduced by correcting the absolute energy scale used to reconstruct the transverse momenta of jets 76$]$. The jet momenta in simulation are also smeared in order to match the jet resolution observed in data. The $\vec{H}_{\mathrm{T}}$ is then recomputed based on the corrected jet momenta.

Further corrections are applied to improve the performance of the $\vec{E}_{\mathrm{T}}$ reconstruction in events that have a large number of additional minimum bias interactions (pileup). The nonlinearity and energy thresholds of the calorimeters induce a bias in the $\vec{E}_{\mathrm{T}}$ calculation in the direction of the vectorial $\vec{p}_{\mathrm{T}}$ sum of neutral particles. This effect is corrected for by parametrizing the induced $\vec{E}_{\mathrm{T}}$ in events with exactly one minimum bias $p p$ interaction. This parametrization is used to calculate a sum over all pileup interactions in a given event which is then subtracted from the reconstructed 
$\vec{E}_{\mathrm{T}}$.

Due to imperfect detector alignment, detector inefficiencies, a residual $\phi$ dependence of the calibration, and a small offset between the center of the detector and the beamline [?], an asymmetry in $\phi$ is observed in the reconstructed $\vec{H}_{\mathrm{T}}$. The observed $\vec{H}_{\mathrm{T}} \phi$ asymmetry increases approximately linearly with the number of pileup interactions, and this dependence is used to derive a correction. Since the detector alignment and $\phi$-intercalibrations are different between data and simulation the corrections are derived separately for data and simulated events. Quadratic functions are used to fit the dependence of $\not_{x}$ and $\not_{y}$ on the number of reconstructed vertices:

$$
\begin{aligned}
& \left\langle\not t_{x}\right\rangle=c_{x_{o}}+c_{x_{s}} \cdot N_{\mathrm{vtx}}+c_{x_{2}} \cdot N_{\mathrm{vtx}}^{2}, \\
& \left\langle\not y_{y}\right\rangle=c_{y_{o}}+c_{y_{s}} \cdot N_{\mathrm{vtx}}+c_{y_{2}} \cdot N_{\mathrm{vtx}}^{2} .
\end{aligned}
$$

The coefficients of the fit are used to correct the $\vec{E}_{\mathrm{T}}$ calculation on an event-by-event basis:

$$
\begin{aligned}
& \not_{x}^{\text {corr }}=\not \phi_{x}-\left\langle\not_{x}\right\rangle=\not \phi_{x}-\left(c_{x_{0}}+c_{x_{s}} \cdot N_{\mathrm{vtx}}+c_{x_{2}} \cdot N_{\mathrm{vtx}}^{2}\right), \\
& \not_{y}^{\text {corr }}=\not \phi_{y}-\left\langle\not_{y}\right\rangle=\not \phi_{y}-\left(c_{y_{0}}+c_{y_{s}} \cdot N_{\mathrm{vtx}}+c_{y_{2}} \cdot N_{\mathrm{vtx}}^{2}\right) .
\end{aligned}
$$

The coefficients are extracted from the fits to the candidate events in data and simulation and are shown in Fig 4.9 and Table 4.7.

Table 4.6: The parameters for the $\overrightarrow{\mathscr{E}}_{\mathrm{T}} \phi$-asymmetry corrections for x-axis projection of PF $\vec{E}_{\mathrm{T}}$ for data and simulation.

\begin{tabular}{|l|c|c|c|}
\hline & $c_{x_{0}}(\mathrm{GeV})$ & $c_{x_{s}}(\mathrm{GeV})$ & $c_{x_{2}}(\mathrm{GeV})$ \\
\hline \hline Data & $-0.19 \pm 0.02$ & $0.31 \pm 0.003$ & $-0.0013 \pm 0.0001$ \\
Simulation & $0.027 \pm 0.03$ & $-0.0058 \pm 0.0045$ & $-0.0009 \pm 0.0001$ \\
\hline
\end{tabular}

Table 4.7: The parameters for the $\vec{E}_{\mathrm{T}} \phi$-asymmetry corrections for y-axis projection of PF $\vec{E}_{\mathrm{T}}$ for data and simulation.

\begin{tabular}{|l|c|c|c|}
\hline & $c_{y_{0}}(\mathrm{GeV})$ & $c_{y_{s}}(\mathrm{GeV})$ & $c_{y_{2}}(\mathrm{GeV})$ \\
\hline \hline Data & $-0.84 \pm 0.02$ & $-0.005 \pm 0.003$ & $-0.002 \pm 0.0001$ \\
Simulation & $-0.031 \pm 0.034$ & $-0.090 \pm 0.004$ & $-0.0009 \pm 0.0001$ \\
\hline
\end{tabular}

The $\phi$ and $\vec{E}_{\mathrm{T}}$ in the event is then recalculated using the corrected $\mathscr{E}_{x}$ and $\mathbb{E}_{y}$. Fig 4.10 shows 

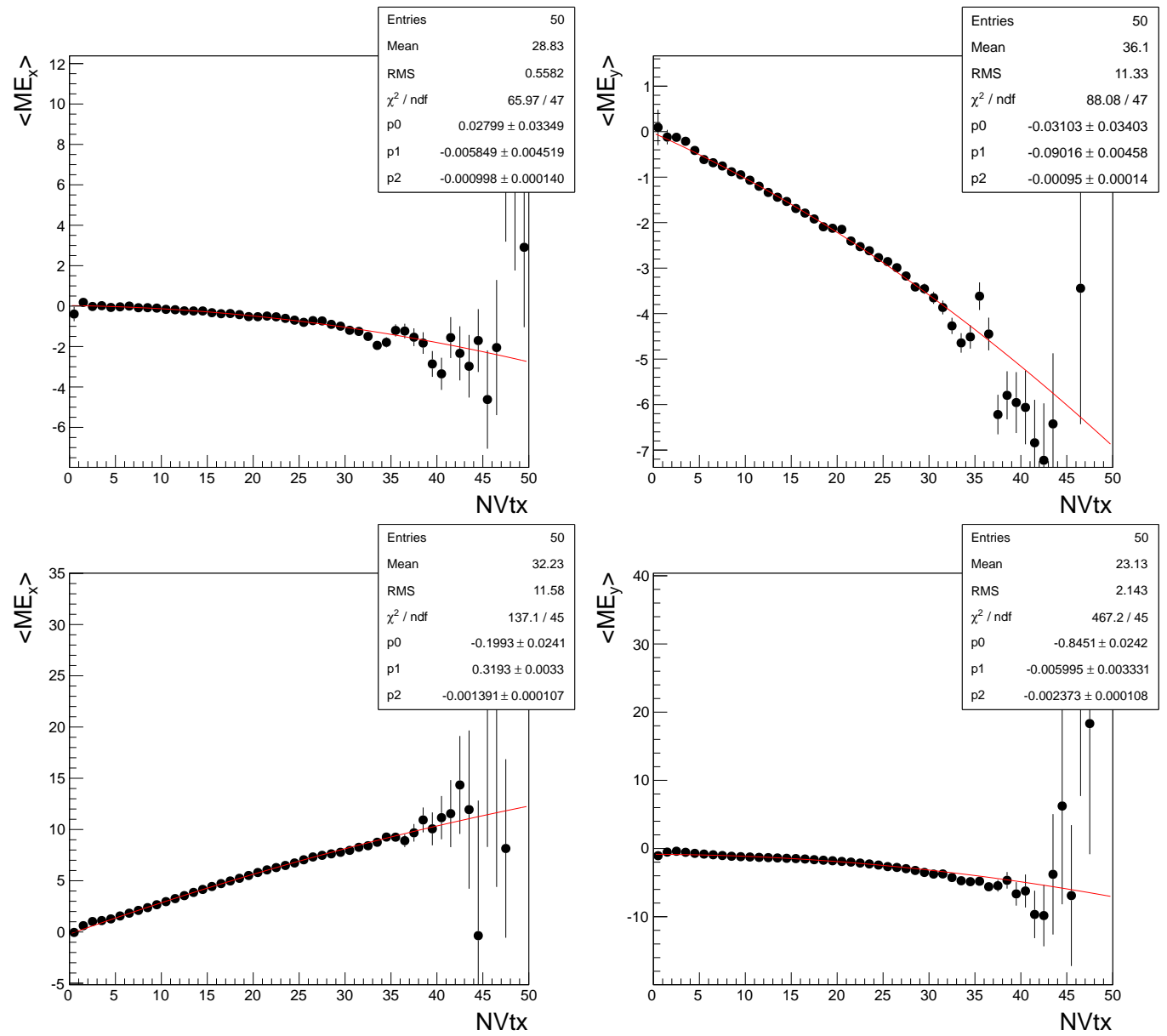

Figure 4.9: MET $\phi$ modulation correction fits for MC simulation (top row), and data (bottom row). 
the $\phi$ distributions for $\vec{E}_{\mathrm{T}}$ before and after the correction.
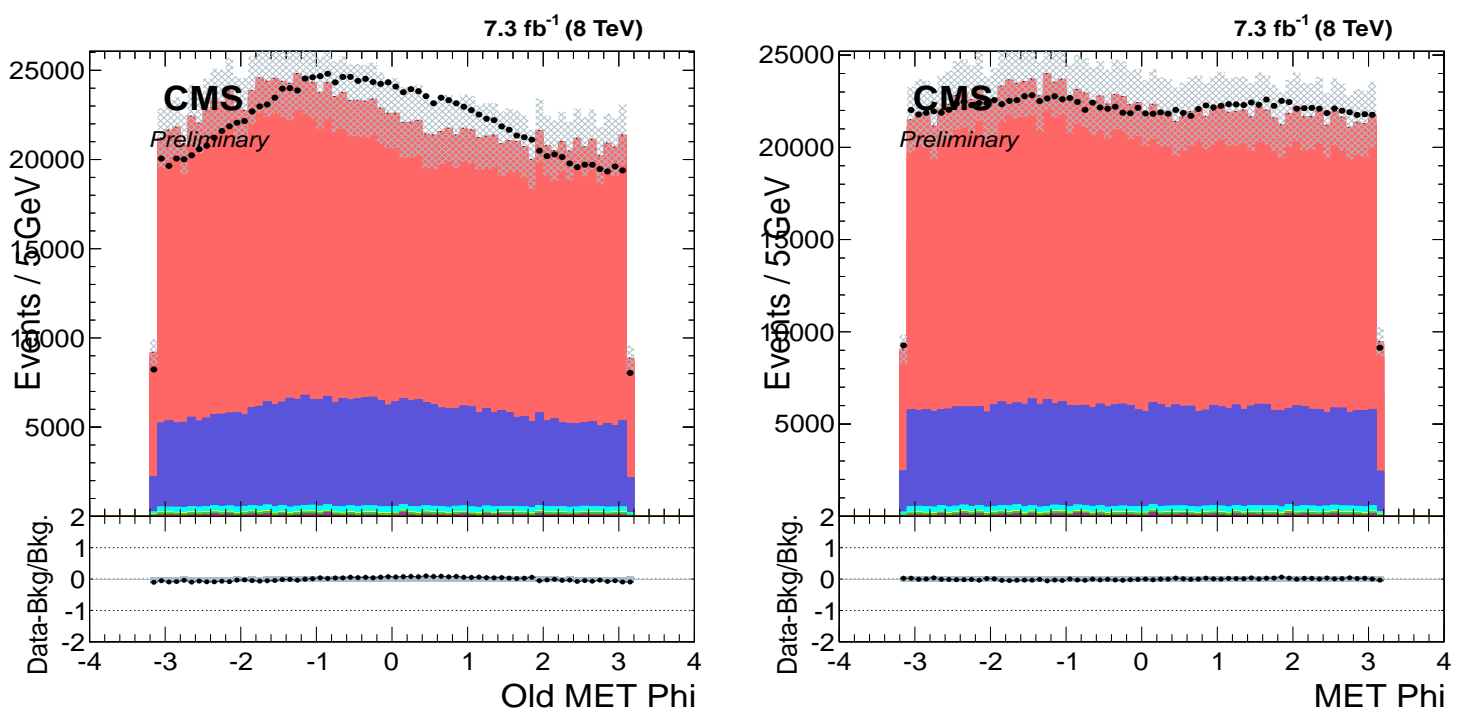

Figure 4.10: $\phi$ distributions before (a) and after (b) the correction in data and simulation.

The $\overrightarrow{\mathscr{H}}_{\mathrm{T}}$ reconstruction is highly sensitive to electronic noise which can produce spurious signals. Several noise event filters are applied to remove such events and ensure good quality of data:

- CSC tight beam halo: rejects events with a secondary particle shower produced due to collisions of the beam with residual gas inside the LHC vacuum chamber.

- HBHE noise filter: removes events with noise in the hybrid photo-diodes (HPTDs), used to convert the scintillator light into an electrical output and the readout boxes (RBXs) which contain them.

- ECAL dead cell trigger primitive (TP) filter: rejects events where the TP $E_{\mathrm{T}}$ at the masked crystal cells exceed $63.75 \mathrm{GeV}$

- HCAL laser filter: rejects events when firing of the HCAL laser overlaps with an LHC bunch crossing.

- Tracking failure filter: rejects events where standard or large calorimeter deposits contrast with a lack of reconstructed tracks.

- Bad EE Supercrystal filter: removes events from two $5 \times 5$ crystals that give anomalously high energy.

- Tracking POG filters: rejects events where no tracks are reconstructed due to aborting of the reconstruction algorithm because of CPU time limitations. 
Even if no genuine $\overrightarrow{\mathscr{H}}_{\mathrm{T}}$ is expected in most physical processes, many physics and detector effects can significantly affect the $\vec{E}_{\mathrm{T}}$ measurement, inducing a non-zero $\vec{E}_{\mathrm{T}}$ calculation in an event. As described above, detector noise, detector inefficiencies, detector energy resolution, and jet energy corrections are the major detector sources of mismeasured $\vec{H}_{\mathrm{T}}$, while pileup interactions, the underlying pp interaction, and variations in jet composition are physical sources of mismeasured $\vec{E}_{\mathrm{T}}$.

Several techniques are available to identify events with mismeasured $\vec{E}_{\mathrm{T}}$, one of which is referred to as the " $\vec{E}_{\mathrm{T}}$ significance", or simply $\mathcal{S}\left[77\right.$. In this method, a p-value that the reconstructed $\vec{E}_{\mathrm{T}}$ is inconsistent with the $\vec{E}_{\mathrm{T}}=0$ hypothesis is evaluated for each event taking as input the reconstructed objects and their resolutions. The significance is defined as the log-likelihood ratio:

$$
\mathcal{S} \equiv 2 \ln \left(\frac{\mathcal{L}\left(\vec{\varepsilon}=\sum \vec{\varepsilon}_{i}\right)}{\mathcal{L}(\vec{\varepsilon}=0)}\right)
$$

The numerator expresses the likelihood of that the true value of the missing transverse energy is equal to the observed value, while the denominator expresses the likelihood of the null hypothesis, that the true missing transverse energy is actually zero. Under the null hypothesis, observation of any non-zero missing transverse energy is attributed to mis-measurement of other objects in the event. A high value of $\mathcal{S}$ is an indication that the $\vec{E}_{\mathrm{T}}$ observed in the event is genuine caused by undetected objects such as neutrinos.

\subsubsection{4 $\not_{T}$ Minimization}

A complementary technique for identifying events with mismeasured $\vec{E}_{\mathrm{T}}$ was developed during the analysis of the $\gamma+\not_{T}$ final state. In this method a $\chi^{2}$ function with the following form is constructed:

$$
\chi^{2}=\sum_{i=\text { objects }}\left(\frac{\left(p_{\mathrm{T}}^{\text {reco }}\right)_{i}-\left(\widetilde{p_{\mathrm{T}}}\right)_{i}}{\left(\sigma_{p_{\mathrm{T}}}\right)^{i}}\right)^{2}+\left(\frac{\widetilde{E}_{x}}{\sigma_{\widetilde{E}_{x}}}\right)^{2}+\left(\frac{\widetilde{E}_{y}}{\sigma_{\widetilde{F}_{y}}}\right)^{2}
$$

In the above equation, $\left(p_{\mathrm{T}}^{r e c o}\right)_{i}$ are the transverse momenta of the reconstructed objects, the $\left(\sigma_{p_{\mathrm{T}}}\right)_{i}$ are the expected resolutions of each object, and the $\left(\widetilde{p}_{\mathrm{T}}\right)_{i}$ are the free parameters allowed to vary in order to minimize the function. Furthermore, $\not_{x}$ and $\not_{y}$ can be expressed as:

$$
\begin{aligned}
& \widetilde{E}_{x, y}=\underbrace{\mathbb{F}_{x, y}^{r e c o}+\sum_{i=o b j e c t s}\left(p_{x, y}^{r e c o}\right)_{i}}_{=0}-\left(\widetilde{p}_{x, y}\right)_{i} \\
& \widetilde{E}_{x, y}=-\sum_{i=\text { objects }}\left(\widetilde{p}_{x, y}\right)_{i}
\end{aligned}
$$


In events with no genuine $\not_{T}$, the mismeasured quantities can be re-distributed back into the particle momenta, resulting in a low $\chi^{2}$ value. On the other hand, in events with genuine $\not_{T}$ from undetected particles, the minimization generally will yield larger $\chi^{2}$ values.

All the visible objects in the hard-scattering event passing the appropriate identification requirements are included in the $\chi^{2}$ function. The jets are required to be coming from the hard scattering, and have $p_{\mathrm{T}}>30 \mathrm{GeV}$, and to not overlap with any photon or a lepton included in the $\chi^{2}$ function. Electrons and muons that are required to not overlap with any photon and to have $p_{\mathrm{T}}>10 \mathrm{GeV}$.

Assignment of the momentum measurement uncertainties is an important aspect of this technique as these uncertainties are needed in Eq. 4.7 to weight the particle momenta according to their resolution. The objects that have the poorest resolution are jets, which means that the dominant source of mismeasured $\not_{T}$ will be mismeasured jets. The jet resolution has been measured using the dijet $p_{\mathrm{T}}$ asymmetry distributions and the $\gamma+$ jet balancing technique 78 . The jet resolution as a function of transverse momentum is given in 12 different $\eta$ ranges as shown in Figs. 4.11 and 4.12 The resolution difference between data and simulation is also taken into account and scale factors are shown in Table 4.8. The functional form used to parametrize the jet resolution is [78]:

$$
J E R=\sqrt{\left(\frac{N}{p_{\mathrm{T}}}\right)^{2}+S^{2} p_{\mathrm{T}}^{m-1}+C^{2}}
$$

where, $N$ refers to the noise, $S$ to the stochastic, and $C$ to the constant terms. The additional parameter $m$ is introduced to improve the quality of the fits.

Table 4.8: Scale Factors for the resolution difference in data and simulations

\begin{tabular}{|c|c|}
\hline$|\eta|$ Bin & Data/MC Ratio ( \pm stat. + syst - syst $)$ \\
\hline \hline $0.0-0.5$ & $1.052 \pm 0.012+0.062-0.061$ \\
$0.5-1.1$ & $1.057 \pm 0.012+0.056-0.055$ \\
$1.1-1.7$ & $1.096 \pm 0.017+0.063-0.063$ \\
$1.7-2.3$ & $1.134 \pm 0.035+0.087-0.085$ \\
$2.3-5.0$ & $1.288 \pm 0.127+0.155-0.153$ \\
\hline
\end{tabular}

The $\not_{x}$ and $\not_{y}$ resolution are also calculated as a function of the $\sum E_{T}-E_{\mathrm{T}}^{\gamma}$ in the event to determine the dependence of the $\not_{T}$ resolution on the calorimeter energy resolution. As described above, the major source of uncertainty in the $\not_{T}$ measurement is due to jets. For this reason, $E_{\mathrm{T}}^{\gamma}$ is subtracted given that it has excellent resolution. The $\mathscr{E}_{x}$ and $\mathscr{E}_{y}$ resolutions are shown in Fig. 4.13 These studies are were performed using sample of $\gamma+$ jets events in data and simulation. The resolution difference between data and $\mathrm{MC}$ simulation is used to derive the scale factors provided in Table 4.9. The functional form used to model the resolutions is: 

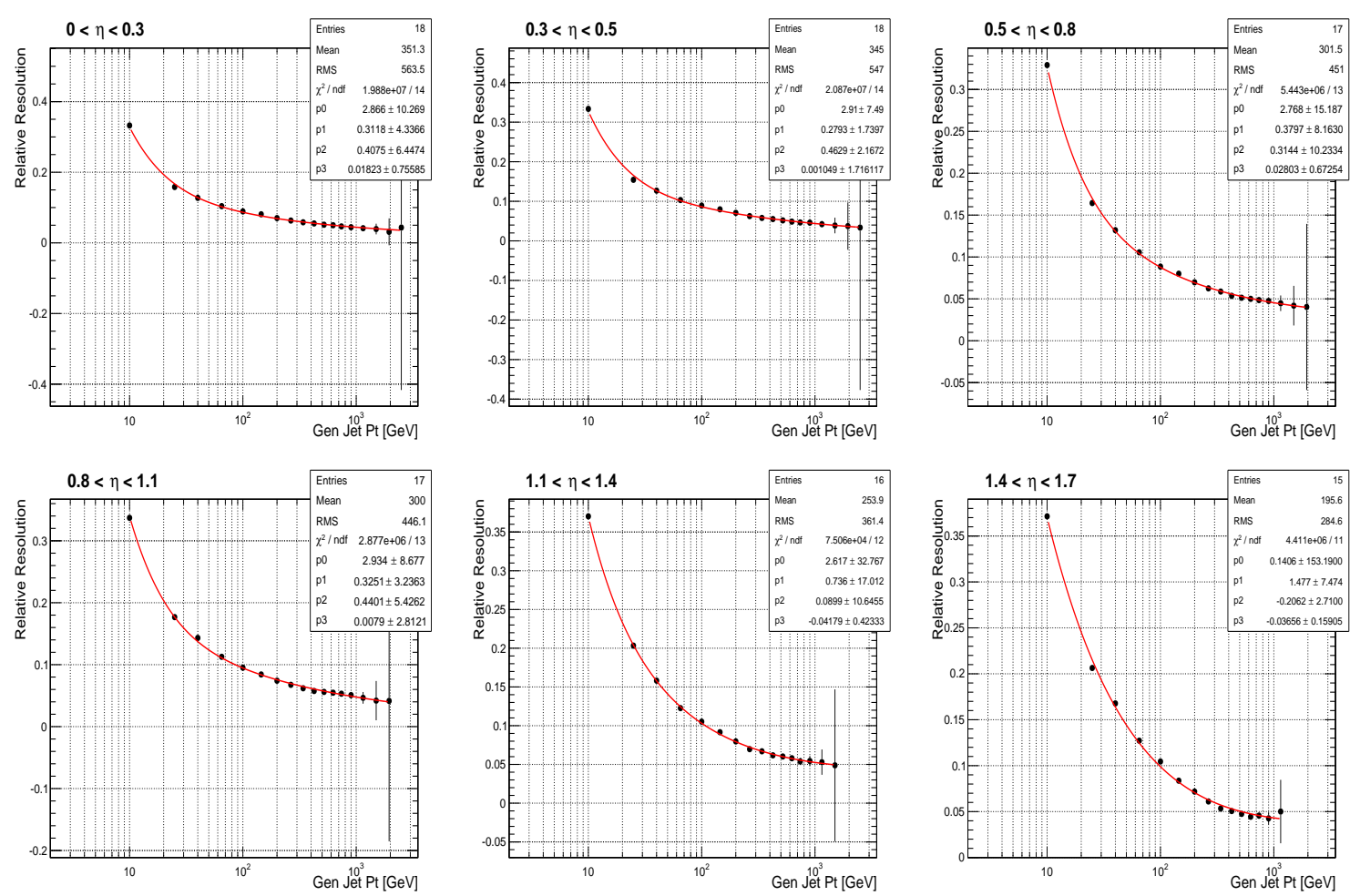

Figure 4.11: The jet transverse momentum resolution as a function of transverse momentum in 12 different $\eta$ ranges (see also Fig. 4.12).

$$
\sigma_{\not H_{T}}=c_{1} \sqrt{\sum \not_{T}-q_{T}}+c_{2}
$$

Table 4.9: Scale Factors for the $\not_{T}$ resolution difference in data and MC simulation using a $\gamma+$ jet sample.

\begin{tabular}{|c|c|c||c|c|}
\hline Component & $\sigma_{0}(\mathrm{GeV})$ & $\mathrm{R}=\sigma_{0}($ data $) / \sigma_{0}(\mathrm{MC})$ & $\sigma_{s}\left(\mathrm{GeV}^{1 / 2}\right)$ & $\mathrm{R}=\sigma_{s}($ data $) / \sigma_{s}(\mathrm{MC})$ \\
\hline \hline$\not_{x}$ & $0.37 \pm 0.42$ & $0.12 \pm 0.14 \pm 0.19$ & $0.61 \pm 0.01$ & $1.15 \pm 0.03 \pm 0.15$ \\
$\not_{y}$ & $0.17 \pm 0.37$ & $0.05 \pm 0.11 \pm 0.13$ & $0.62 \pm 0.01$ & $1.17 \pm 0.03 \pm 0.16$ \\
\hline
\end{tabular}

Two variables are identified, which give good discrimination between signal-like and backgroundlike events. These variables are the probability value of the $\chi^{2}$ function $\left(\operatorname{Prob}\left(\chi^{2}\right)\right)$ and the recalculated minimized $\not_{T}$. Both of these variables are found to have a good agreement between data and simulation and their distributions are shown in Fig. 4.14. It should be noted that the flatness of the $\operatorname{Prob}\left(\chi^{2}\right)$ distribution would serve as an excellent cross-check of modeling of the resolutions. Given that the distribution is mostly flat (with peaks coming from non-Gaussian tails of the resolution), it serves as a confirmation that the estimated resolutions are good approximations. 

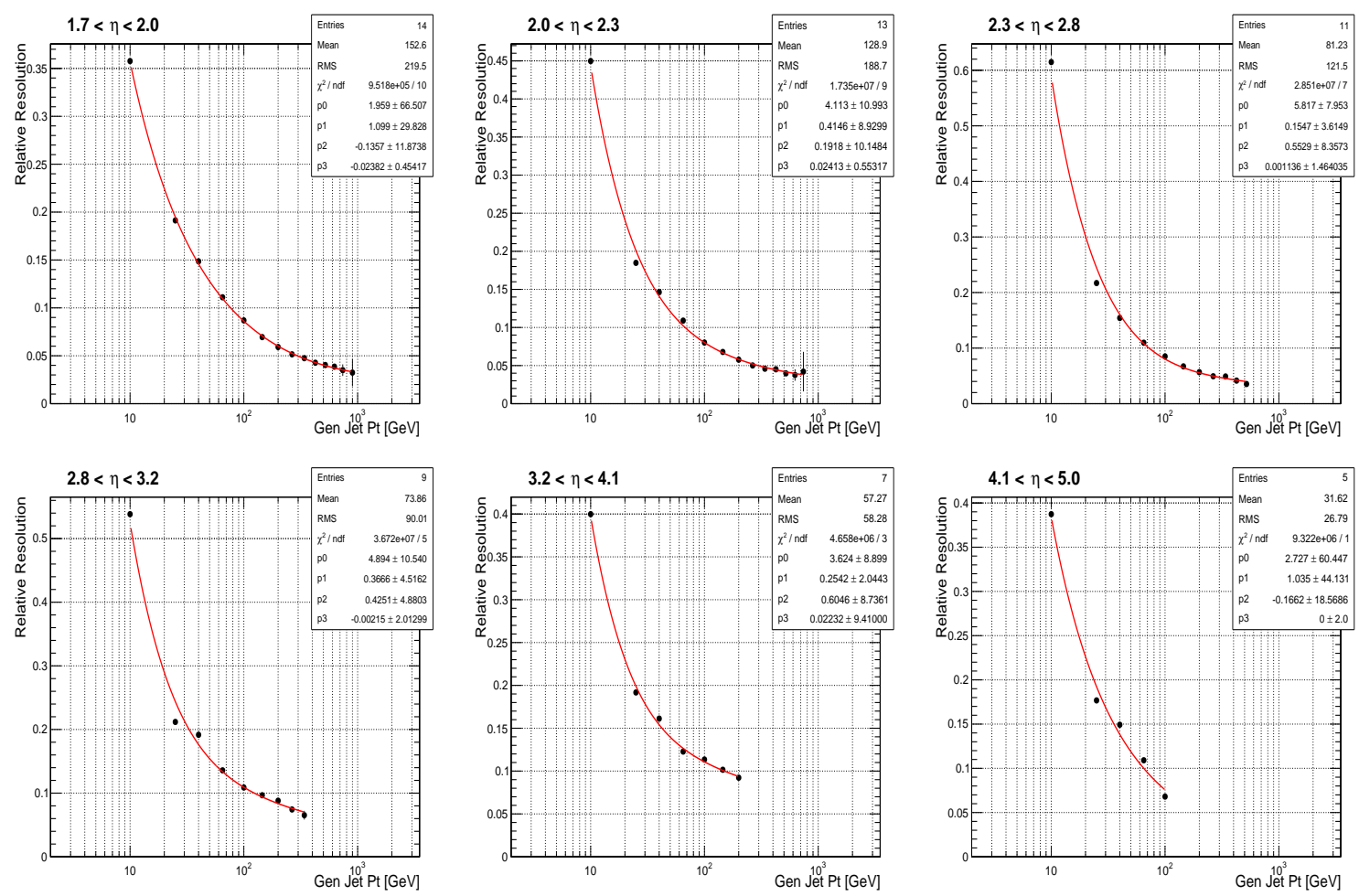

Figure 4.12: The jet transverse momentum resolution as a function of transverse momentum in 12 different $\eta$ ranges (continued, see also Fig. 4.11)
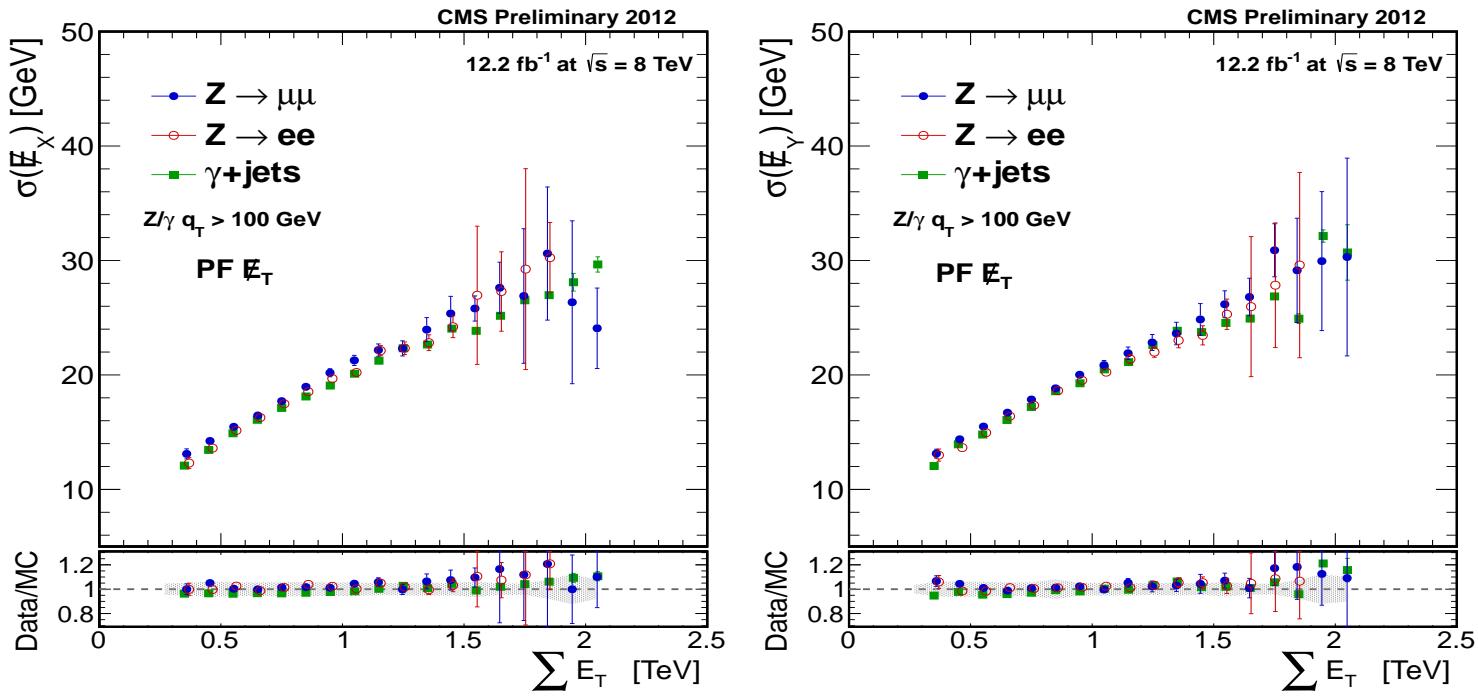

Figure 4.13: The $E_{x, y}$ resolution as a function of $\sum E_{T}$. 

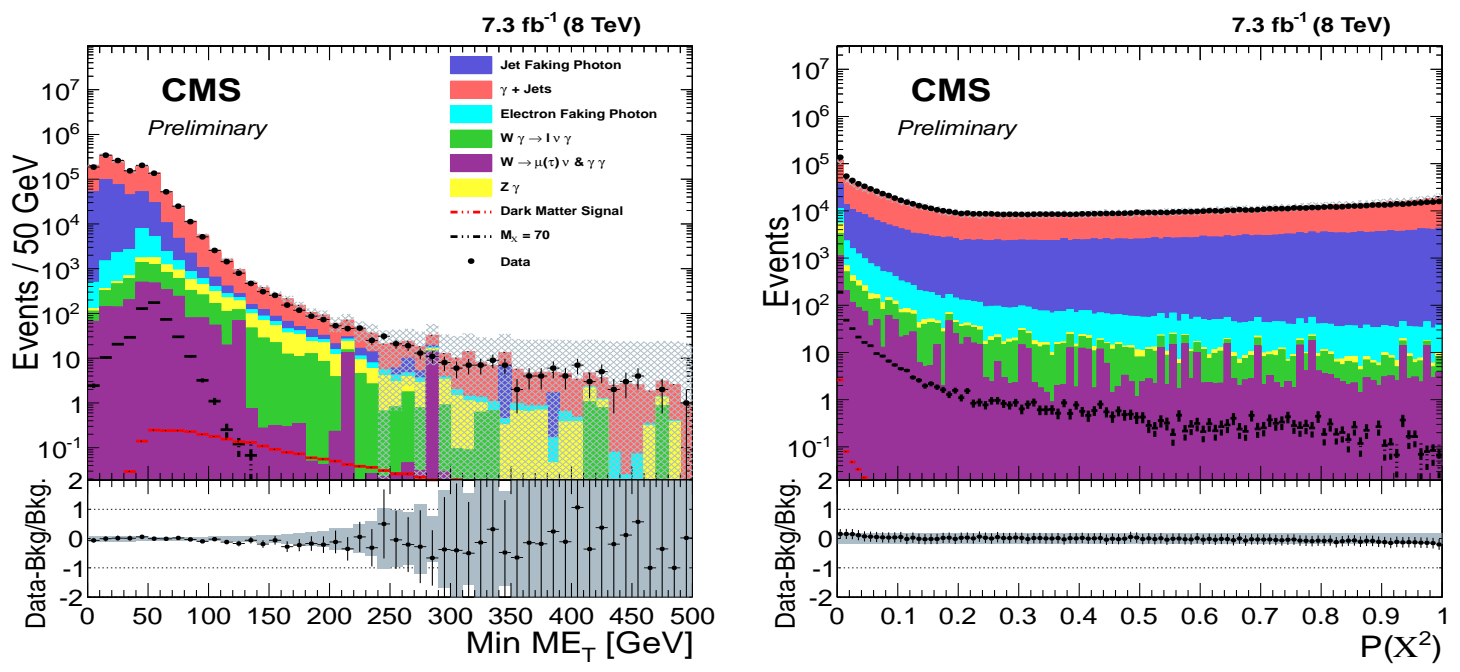

Figure 4.14: The probability value of the $\chi^{2}$ function and the recalculated minimized $\not_{T}$ distributions in data and simulation. 


\section{Chapter 5}

\section{Search for new physics in final}

\section{states with photon and missing}

\section{transverse energy}

In this chapter, a dataset collected by the CMS experiment at the LHC consisting of events with a low transverse energy photon $(\gamma)$ and low missing transverse energy $\left(\not \phi_{T}\right)$ (also called the "monophoton" final state) is used to constrain a variety of extensions of the standard model, including the SUSY model described in Section 2.2.1.

\subsection{Signal Modeling}

As described in Section 2.2.1 in SUSY scenarios where the SUSY breaking scale is low $(\sqrt{f} \sim \mathrm{TeV})$ the recently discovered Higgs boson $\left(m_{h}=125 \mathrm{GeV}\right)$ 1,2 may decay into a gravitino $(\widetilde{G})$ and neutralino $\left(\widetilde{\chi}_{1}^{0}\right)$, with the neutralino subsequently decaying into a gravitino and a photon 79 . In this model, the gravitino is the LSP and the neutralino is the next-to-lightest supersymmetric particle (NLSP). Fig. 5.1 shows the Feynman diagram for this process.

This decay mode produces a single isolated photon and $\not_{T}$ from the undetected gravitinos. If $m_{\widetilde{\chi}_{1}^{0}}<m_{h} / 2$, the decay process $h \rightarrow \widetilde{\chi}_{1}^{0} \widetilde{\chi}_{1}^{0} \rightarrow \gamma \gamma+\not_{T}$ would dominate. Therefore the kinematic region of interest for this search is $m_{h} / 2<m_{\widetilde{\chi}_{1}^{0}}<m_{h}$. Furthermore, since $m_{h}=125 \mathrm{GeV}$, the $E_{\mathrm{T}}^{\gamma}$ and $\not_{T}$ will be relatively low.

The results of the search are presented in terms of the low-scale SUSY breaking model for 


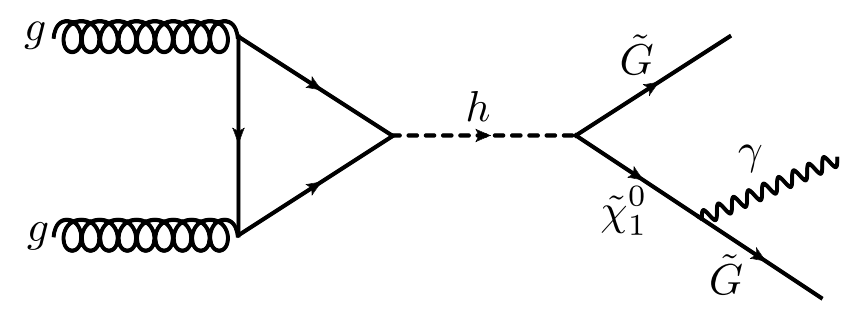

Figure 5.1: Feynman diagrams of a Higgs boson decay to gravitino LSP and a neutralino NLSP, which subsequently decays to a gravitino and photon.

$m_{h}=125 \mathrm{GeV}$ and $m_{\chi_{1}^{0}}$ between 95 and $120 \mathrm{GeV}$, and for $m_{h}$ between 125 and $400 \mathrm{GeV}$ for $m_{\chi_{1}^{0}}=m_{h}-30 \mathrm{GeV}$. The effect of a non-zero $\chi_{1}^{0}$ lifetime is studied for the case where $m_{h}=125$ $\mathrm{GeV}$ and $m_{\chi_{1}^{0}}=95 \mathrm{GeV}$.

The signal processes are generated using the MADGRAPH 5.1 event generator 80 and are interfaced using the PYтнIA 6.4 generator 81 for the parton shower and hadronization. The set of parton distribution functions (PDF) used is CTEQ6L 82] for leading-order (LO) generators. For all processes, the detector response is simulated using a detailed description of the CMS detector, based on the GEANT4 package 83 .

\subsection{Event Selection}

Events for this analysis are collected using a single HLT path, which is seeded by either a $20 \mathrm{GeV}$ or a $22 \mathrm{GeV}$ single e/ $\gamma \mathrm{L} 1$ trigger depending on the running period. The HLT is used to select events with at least one photon with $E_{\mathrm{T}}>30 \mathrm{GeV}$ within the ECAL barrel region $\left(\left|\eta^{\gamma}\right|<1.44\right)$ and calorimetric $\not_{T}>25 \mathrm{GeV}$ with additional selections to suppress the anomalous noise in the HCAL barrel (HB) and endcap (HE) subdetectors due to characteristics of the hybrid photodiodes and the readout boxes 84 . The trigger further requires the photon to pass a loose calorimeterbased isolation selection and to exhibit shower shape characteristics consistent with unconverted photons. The main shower shape requirement is based on the $R_{9}$ variable, defined as the ratio of the energy deposited in a $3 \times 3$ crystal region centered around the crystal containing an energy deposit greater than all of its immediate neighbors (the "seed crystal") to the energy of the entire deposit of the photon ("supercluster"). The data recorded with this trigger corresponds to an integrated luminosity of $7.3 \mathrm{fb}^{-1}$ and was part of the CMS "data parking" program in 2012. With the data parking program, CMS recorded additional data with relaxed trigger requirements planning for a delayed offline reconstruction in 2013 after the completion of the LHC Run I.

In the offline selection, the events are required to have at least one well-identified vertex with a 
distance less than $24 \mathrm{~cm}$ away from the nominal interaction point in $z$-direction and $2 \mathrm{~cm}$ away in the $x y$-plane. The vertex corresponding to the origin of the hard-scattering process with the largest value of $\sum p_{T}^{2}$ of all associated tracks is identified as the primary vertex. Each selected event is required to have at least one photon candidate with $E_{\mathrm{T}}^{\gamma}>45 \mathrm{GeV}$ and $\left|\eta^{\gamma}\right|<1.44$ fulfilling the medium identification and isolation requirements. The photon with the highest $E_{\mathrm{T}}$ in the event that satisfies all of the requirements is selected as the photon candidate for the signal sample. Anomalous signals in the ECAL, due to direct interaction of particles with the ECAL photodiodes, are rejected using additional shower shape requirements on the $\eta$ and $\phi$ width of the shower. In addition, we reject showers that deposit more than $95 \%$ of their energy in the seed crystal 85 . Finally, the PF $\not_{T}$ is required to be greater than $40 \mathrm{GeV}$.

To reduce the SM backgrounds arising from the leptonic decays of $\mathrm{W}$ and $\mathrm{Z}$ bosons, a lepton veto is applied. Events are rejected if they have at least one electron fulfilling cut based loose identification requirement 74 with $p_{\mathrm{T}}^{e}>10 \mathrm{GeV}$ and $\left|\eta^{e}\right|<2.5$ (excluding the transition region of $\left.1.44<\left|\eta^{e}\right| \leq 1.55\right)$ and are outside the cone defined by $\Delta R=0.3$ around the photon candidate. Muons candidates, which are identified using the PF algorithm using hits in the tracker and the muon systems, are required to have $p_{\mathrm{T}}^{\mu}>10 \mathrm{GeV},\left|\eta^{\mu}\right|<2.1$, and $\Delta R(\gamma, \mu)>0.3$ separation from the photon candidate. Events are rejected if any such muon is present in the event.

This level of selection is referred to as the preselection and is applied for both the model independent analysis and the analysis of the SUSY benchmark model. The additional selection requirements which are applied differ between the two analyses.

To define the jet candidates, identification criteria are used to separate pileup jets from the jets originating from hard scattering. These identification criteria are based on the trajectory of tracks associated with the jets inside the tracker volume, the topology of the jet shape and multiplicity of the objects constituting these jets 86 . Only jets with $p_{\mathrm{T}}^{\text {jet }}>30 \mathrm{GeV}$ and $\left|\eta^{\text {jet }}\right|<2.4$ and that fulfill the non-pileup identification requirements are considered in the event. These jets must not overlap with photon candidate within $\Delta R(\gamma$,jet $)<0.5$. In the model independent analysis, events with two or more jets are rejected and, if there is a jet in the event, we also require that $\Delta \phi(\gamma$, jet $)<2.5$.

In the analysis of the SUSY benchmark model, where no requirement is made on the jet multiplicity, more advanced selection is applied to reduce the background due to mismeasured $\not_{T}$. Mismeasured $\not_{T}$ can arise from many sources, including limited $\not_{T}$ resolution, reconstruction and instrumental inefficiencies, and improper pattern recognition. Due to their large cross section the $\gamma+$ jets and multijet processes can contribute significantly to the background of this analysis, even though such events do not have genuine $\not_{T}$. In order to minimize the contribution from these 
processes, we have used two different methods for identifying events with mismeasured $\not_{T}$. The first one is the $\not_{T}$ significance method 87, which takes into account the reconstructed objects in each event and their known measurement resolutions to compute an event-by-event estimation of the likelihood that the observed $\not_{T}$ is consistent with zero. To complement this method we further developed the missing $H_{\mathrm{T}}\left(\mathrm{M} H_{\mathrm{T}}\right)$ minimization method as described in earlier section. For the analysis of the SUSY benchmark model, the re-calculated $\widetilde{\mathbb{H}_{T}}=\sqrt{\widetilde{\mathscr{E}}_{x}^{2}+\widetilde{\mathscr{H}}_{y}^{2}}$, i.e., in which the original object momenta are replaced with those obtained with the $\chi^{2}$ minimization, is required to be $>45 \mathrm{GeV}$ and the probability value of $\widetilde{\mathscr{H}_{T}}$ to be consistent with mismeasurement obtained from the $\chi^{2}$ minimization is required to be less than $10^{-3}$.

These working points have been chosen after examining the unity normalized distributions of the variables between signal like $(Z \nu \nu \gamma)$ and background like $(\gamma+j e t s)$ event. The Fig. 5.2 shows the two discriminator's distributions. In the $\widetilde{\not_{T}}$ distribution we see that photon + jet background (where the $\not_{T}$ is due to mismeasurements) peaks closer to 0 , where the $Z \nu \nu \gamma$ background peaks around $45 \mathrm{GeV}$ which is due to the selection on the photon $E_{\mathrm{T}}$ at $45 \mathrm{GeV}$. Similarly the $\operatorname{Prob}\left(\chi^{2}\right)$ distribution shows that for events with genuine $\not_{T}$ the minimization results in very low values where for events with mismeasured $\not_{T}$, the probability distribution is mostly flat.

To further suppress multijet backgrounds, events are rejected if the scalar sum of transverse momenta of the identified jets $\left(H_{\mathrm{T}}\right)$ in the event is required to be greater than $100 \mathrm{GeV}$. An additional requirement is made on the angle $(\alpha)$ between the beam direction and the major axis of the supercluster in order to reject photons that have showers elongated along the beam line which is characteristic of non-prompt photons.

Finally, the transverse mass, $M_{T}=\sqrt{2 p_{T}^{\gamma} \not \phi_{T}(1-\cos \Delta \phi)}$, formed by the photon candidate, $\not_{T}$ vector, and the angle $\Delta \phi$ between them, is required to be greater than $100 \mathrm{GeV}$. In order to easily interpret the results within the chosen benchmark model, we require the $E_{\mathrm{T}}^{\gamma}<60 \mathrm{GeV}$.

It should be noted that photon identification working point, along with the $M_{T}$ selection have been studied extensively. The selection has been optimize to maximize the $\mathrm{S} / \sqrt{S+B}$. Working point for the $\not_{T}$ significance selection has been also optimized with respect to the expected limits. Fig. 5.3. shows the details of the optimization.

The final list of advanced selections used in both the model independent analysis and the analysis of the SUSY benchmark model, with the relative cumulative efficiencies of the selection requirements relative to the preselection, is given in Table 5.1 . 

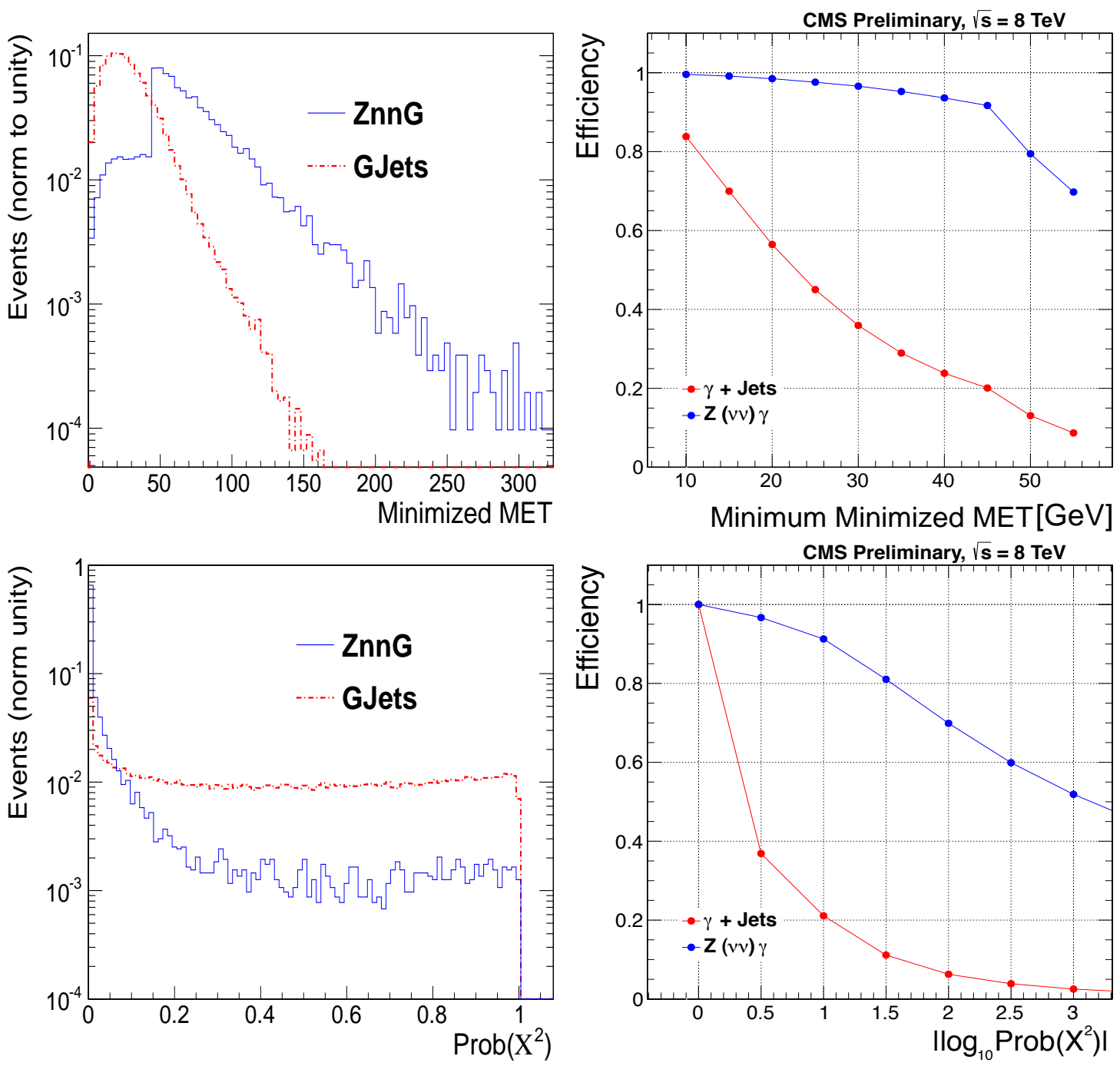

Figure 5.2: The shape difference between signal $Z \nu \nu \gamma$ (blue) and background $\gamma+$ jets (red) like processes, along with the performance curves.

\subsection{Background Modeling}

The irreducible background for the $\gamma+\not_{T}$ signal is the SM process $Z \gamma \rightarrow \nu \bar{\nu} \gamma$. Reducible SM backgrounds include $W \gamma, W \rightarrow \mathrm{e} \nu, W \rightarrow \mu \nu, W \rightarrow \tau \nu, \gamma+$ jet, multijet (referred to as QCD background), and diphoton events. Background events that do not originate from collision processes are also considered. These backgrounds can be divided broadly into three categories, based on how their contributions are estimated:

- Backgrounds estimated with Monte Carlo (MC) simulation: $Z \gamma \rightarrow \nu \bar{\nu} \gamma, Z \gamma \rightarrow \ell \ell \gamma, W \gamma$, $\gamma+$ jets, $\gamma \gamma$

- Collision backgrounds estimated using data: jets misidentified as photons and electrons 

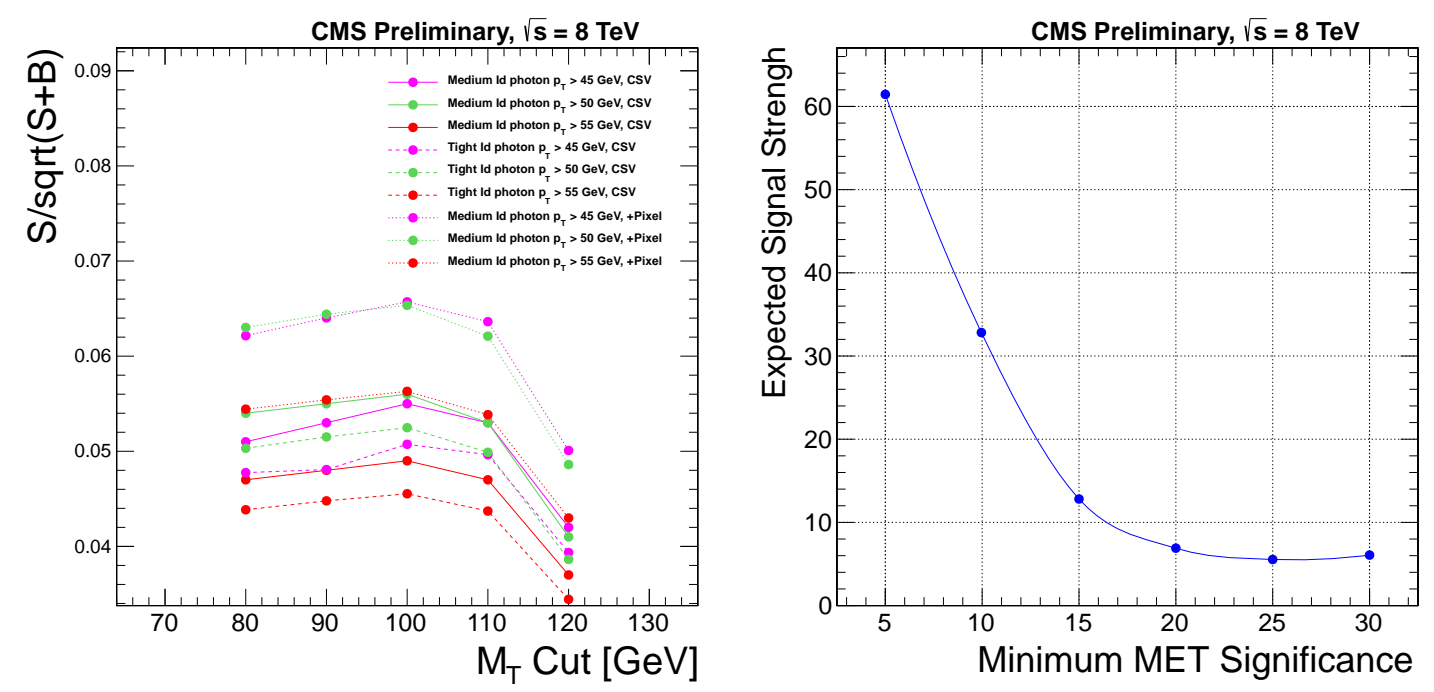

Figure 5.3: The optimization plots on photon energy working point, MT and Met Significance.

Table 5.1: Summary of selection for both model independent analysis and analysis with SUSY benchmark model with the cumulative efficiencies of the selection requirements relative to the preselection for $Z \gamma \rightarrow \nu \bar{\nu} \gamma, \gamma+$ jet and $M_{\widetilde{\chi}_{1}^{0}}=120 \mathrm{GeV}$.

\begin{tabular}{|c|c|c|c|c|c|}
\hline Selection requirements & Model Independent & \multicolumn{3}{|c|}{ SUSY benchmark model } \\
\hline \hline Advanced selection & $Z \gamma \rightarrow \nu \bar{\nu} \gamma$ & $\gamma+$ jet & $Z \gamma \rightarrow \nu \bar{\nu} \gamma$ & $\gamma+$ jet & $M_{\widetilde{\chi}_{1}^{0}}=120 \mathrm{GeV}$ \\
\hline \hline Number of jets $<2$ & 0.909 & 0.769 & - & - & - \\
\hline$\Delta \phi(\gamma$, jet $)<2.5$ & 0.834 & 0.262 & - & - & - \\
\hline Transverse mass $>100 \mathrm{GeV}$ & - & - & 0.867 & 0.292 & 0.829 \\
\hline$H_{\mathrm{T}}<100 \mathrm{GeV}$ & - & - & 0.785 & 0.188 & 0.804 \\
\hline $\mathrm{M} H_{\mathrm{T}}$ minimization: $\widetilde{H}_{T}>45 \mathrm{GeV}$ & - & - & 0.761 & 0.071 & 0.743 \\
\hline $\mathrm{M} H_{\mathrm{T}}$ minimization: Prob $\left(\chi^{2}\right)<10^{-3}$ & - & - & 0.626 & 0.033 & 0.467 \\
\hline$\not_{T}$ significance $>20$ & - & - & 0.440 & 0.001 & 0.195 \\
\hline$\alpha>1.2$ & - & - & 0.390 & 0.001 & 0.165 \\
\hline$E_{\mathrm{T}}^{\gamma}<60 \mathrm{GeV}$ & - & - & 0.074 & 0.0002 & 0.106 \\
\hline
\end{tabular}

misidentified as photons;

- Non-collision backgrounds.

\subsubsection{Simulation-Based Backgrounds}

The $Z \gamma \rightarrow \nu \bar{\nu} \gamma$ is the irreducible background in this study. It is estimated from a MC sample generated with MADGRAPH 88] and processed with PYTHIA v6.426 81 for showering and hadronization. To more accurately describe the topology of these events, up to two extra partons were included in the matrix elements during event generation. The final event yields are normalized using the next-to-leading-order (NLO) cross section calculated with the MCFM event generator 89]. In order to assess the uncertainty on the NLO cross section calculation, four different dynamical energy scales 
were used:

- $\operatorname{sqrt}\left(\mathrm{M}^{2}+\mathrm{pt}_{5}^{2}\right) \rightarrow \sqrt{M_{Z}^{2}+p_{T, \gamma}^{2}}$;

- $\mathrm{m}(345) \rightarrow\left(p_{\nu}+p_{\bar{\nu}}+p_{\gamma}\right)^{2}$

- $\operatorname{pt}($ photon $) \rightarrow p_{T, \gamma}$;

- $\mathrm{HT} \rightarrow p_{T, \nu}+p_{T, \bar{\nu}}+p_{T, \gamma}$.

Additionally, to correctly account for the systematic uncertainty due to the parton distribution function (PDF) choice and the uncertainty in the measured value of the strong coupling $\alpha_{s}\left(m_{Z}\right)$, the prescriptions of the PDF4LHC Working Group 90] were followed. In particular, an envelope technique was used to conservatively combine the $\mathrm{PDF}+\alpha_{S}$ systematics coming from three PDF sets (CT10, MSTW2008, and NNPDF21) $)^{1}$

The resulting cross sections using different dynamical scales along with the statistical and systematic uncertainties related to the $\alpha_{S}$ determination and the choice of PDF are shown in Table 5.2 .

Table 5.2: Cross sections and their uncertainties for the $\mathrm{Z}(\nu \bar{\nu})+\gamma$ (left) process calculated using MCFM.

\begin{tabular}{|c|c|}
\hline Dynamic scale & Central Value \pm Stat \pm Sys $(\mathrm{fb})$ \\
\hline \hline$H_{T}$ & $31972.5 \pm 435.8 \pm 1229.0$ \\
\hline sqrt $\left(\mathrm{M}^{2}+\mathrm{pt}_{5}^{2}\right)$ & $23683.5 \pm 405.5 \pm 1188.2$ \\
\hline $\mathrm{m}(345)$ & $34924.2 \pm 357.5 \pm 1285.7$ \\
\hline $\mathrm{pt}($ photon$)$ & $38024.4 \pm 403.1 \pm 1576.7$ \\
\hline
\end{tabular}

The $W \gamma$ MC simulation was also produced with MADGRAPH, including up to two extra partons in the matrix elements, and normalized using the NLO cross section calculated from MCFM. The resulting cross sections using different dynamical scales along with the statistical and systematic uncertainties related to $\alpha_{S}$ and the choice of PDF are shown in Table 5.3 .

\footnotetext{
${ }^{1}$ We denote $X_{0}^{(i)}$ as the central values of $i=\mathrm{CT} 10$, MSTW, NNPDF as calculated by MCFM, with $\sigma_{P D F+\alpha}^{ \pm(i)}$ their uncertainty. A conservative prediction can be made by computing the maximum and minimum of the combined $\mathrm{PDF}+\alpha_{s}$ uncertainties and defining a mid-point as being halfway in between these values:

$$
\begin{aligned}
U & =\max _{i}\left\{X_{0}^{(i)}+\sigma_{P D F+\alpha_{s}}^{+(i)}\right\} \\
L & =\min _{i}\left\{X_{0}^{(i)}-\sigma_{P D F+\alpha_{s}}^{-(i)}\right\} \\
M & =\frac{U+L}{2}
\end{aligned}
$$

with $U, L$ the upper and lower edges of the envelope and $M$ their mid-point. 
Table 5.3: Cross sections and their uncertainties for the $\mathrm{W}(\ell \nu)+\gamma$ process calculated using MCFM.

\begin{tabular}{|c|c|}
\hline Dynamic scale & Central Value \pm Stat \pm Sys $(\mathrm{fb})$ \\
\hline \hline$H_{T}$ & $561301.2 \pm 17510.0 \pm 19292.4$ \\
\hline sqrt $\left(\mathrm{M}^{2}+\mathrm{pt}_{5}^{2}\right)$ & $452394.4 \pm 14924.8 \pm 16069.3$ \\
\hline $\mathrm{m}(345)$ & $599252.8 \pm 26582.3 \pm 22515.3$ \\
\hline $\mathrm{pt}($ photon$)$ & $455729.8 \pm 15473.6 \pm 18160.9$ \\
\hline
\end{tabular}

The $\gamma+$ jet is one of the most significant backgrounds in this analysis due to the presence of a genuine photon and the large production cross section. The choice of the generator for this background has been studied extensively. Since MADGRAPH produces a more accurate description of the jet multiplicity, it is chosen as the default generator for the $\gamma+$ jet process. The jet multiplicity distribution in simulation is shown in Fig. 5.4
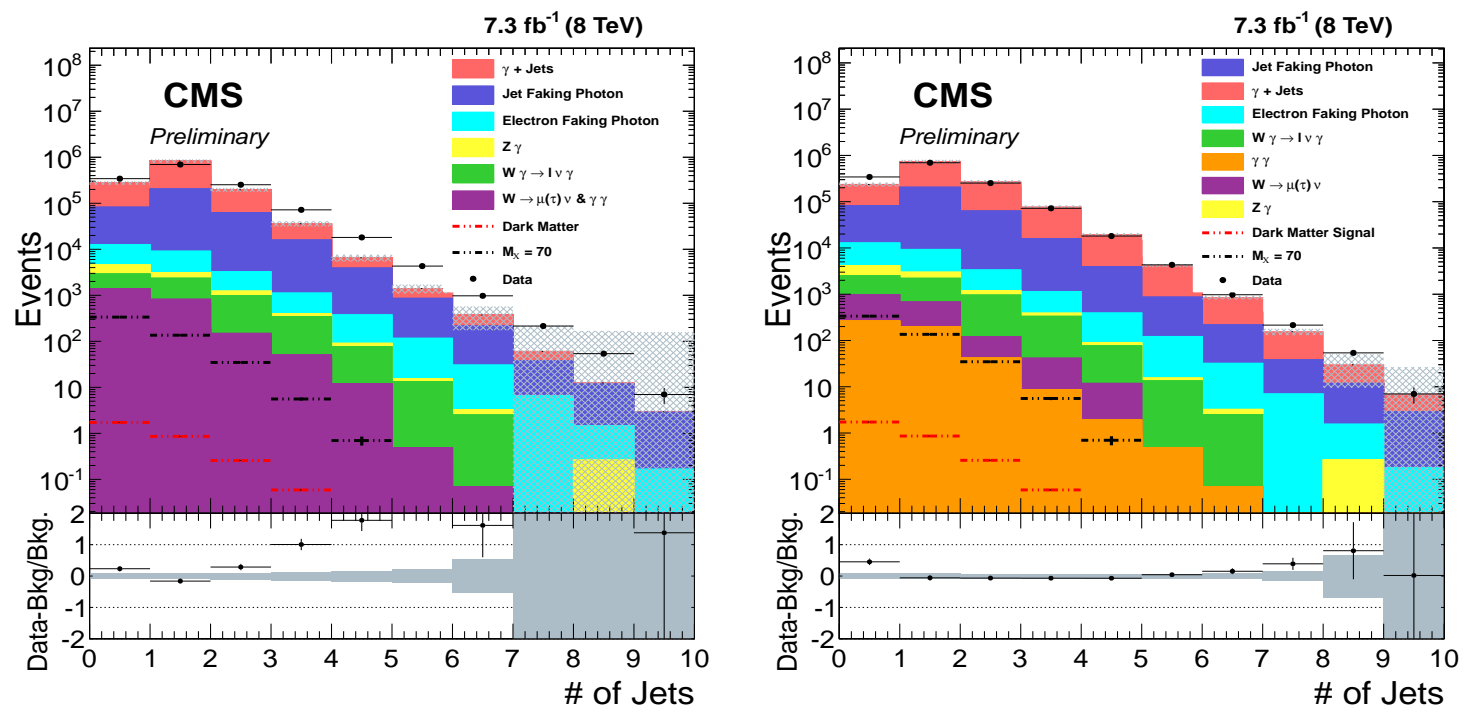

Figure 5.4: Number of Jets above $30 \mathrm{GeV}$ for Pythia (left) and Madgraph (right) simulation samples

The events for this process simulated using MADGRAPH are processed with PYTHIA for showering and hadronization and normalized using the LO cross section from MADGRAPH. The normalization is then corrected using a control sample enriched in $\gamma+$ jet events in data for two different event classes: no-jet and one or more jets. The control sample in data is obtained using events collected with the prescaled single-photon trigger and with the $\not_{T}$ requirement reversed to ensure orthogonality to the signal phase space. The $E_{\mathrm{T}}^{\gamma}$ and jet multiplicity distributions in the control region can be seen in Fig. 5.5 . 

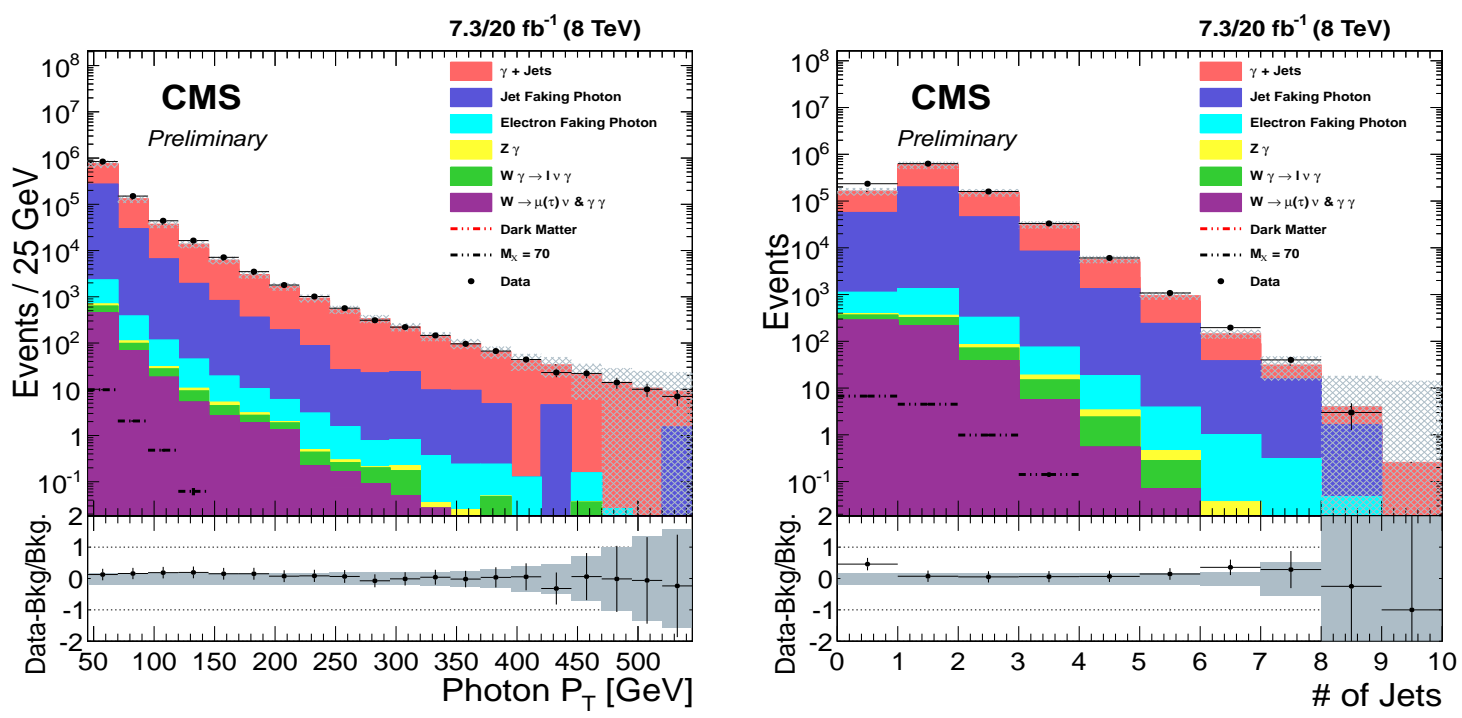

Figure 5.5: The $E_{\mathrm{T}}^{\gamma}$ (left) and $\mathrm{N}$ (jets) (right) distributions in the $\gamma+$ jet control region.

Correction factors for the $\gamma+$ jet normalization $(C)$ are derived by normalizing the event yield in the simulation to match the data in the control region, separately for events with no jets $(C=1.7)$ and one or more jets $(C=1.1)$. These correction factors are then used to normalize the $\gamma+$ jet event yield from simulation in the signal region. An uncertainty of $16 \%$ is derived for these correction factors based on the difference between the corrected and uncorrected simulation and the relative fraction of zero-jet events ( $\sim 10 \%$ of the events in the control region) and events with one or more jets.

The background estimates for the process $Z \gamma \rightarrow \ell \ell \gamma$ is obtained using MADGraPH, and the background estimates for the processes $W \rightarrow \mu \nu, W \rightarrow \tau \nu$ are obtained using PYTHIA. These processes are found to contribute a small fraction of the total background prediction.

All simulated samples were generated with additional minimum bias interactions (pileup) to match the expected conditions of each data taking period. The deterministic annealing primary vertex $(\mathrm{PV})$ reconstruction is well-behaved for high levels of pile up, however the final distribution for the number of reconstructed primary vertices is still sensitive to the details of the primary vertex reconstruction and to differences in the underlying event in data and simulation. The number of reconstructed vertices can further be affected by the event selection criteria and by the trigger.

In order to factorize these effects, instead of reweighting the $\mathrm{MC}$ by the number of reconstructed primary vertices, the simulation is instead reweighted using the number of pileup interactions from the simulation truth. The target pileup distribution for data is derived by using the instantaneous luminosity per bunch crossing together with the total pp inelastic cross-section to generate an ex- 
pected pileup distribution. The distribution of the number of reconstructed vertices in data and simulation is shown in Fig. 5.6 after the reweighting has been applied.
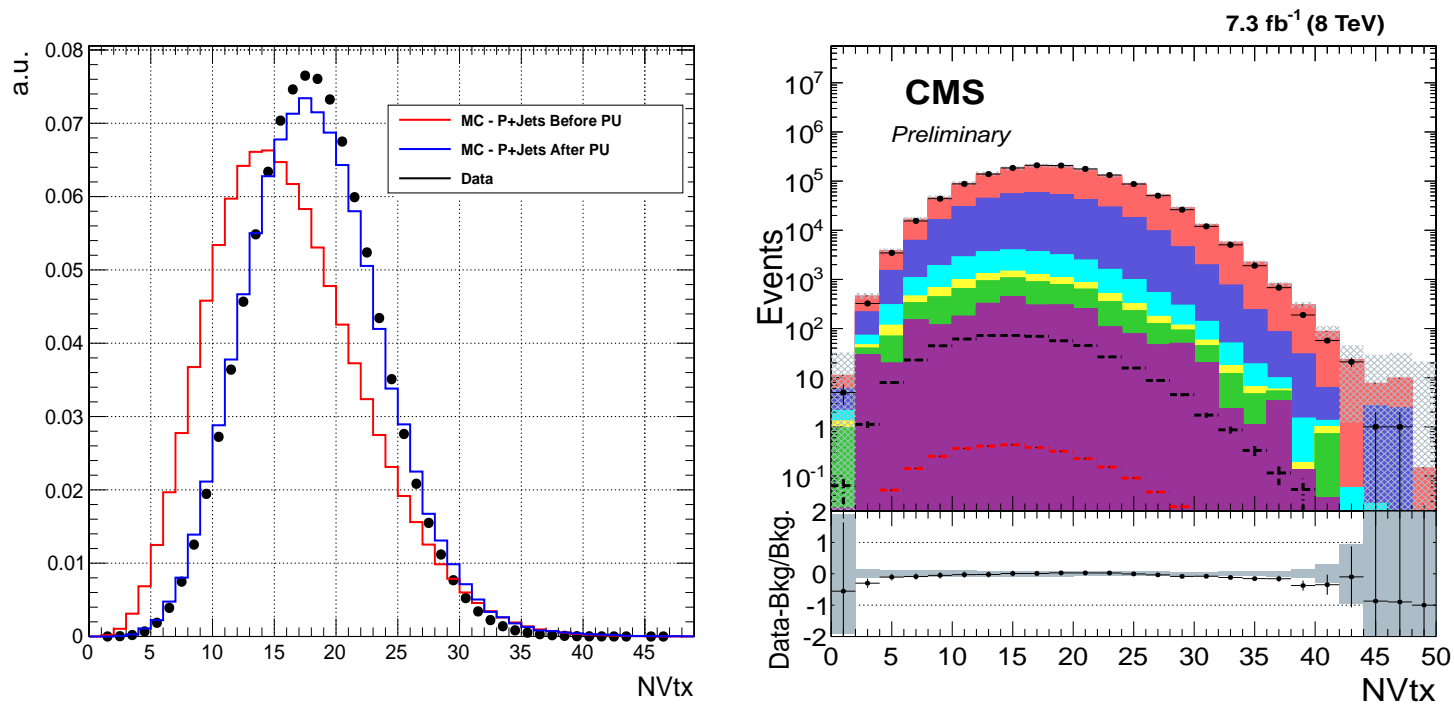

Figure 5.6: (a) Unity-normalized distributions of the number of reconstructed vertices in data and $\gamma+$ jet MC before and after the pileup reweighting. (b) Distribution of the number of vertices after the analysis selection for observed data and predicted background.

\subsubsection{Backgrounds Determined from Data}

\subsubsection{Estimation of electron $\rightarrow$ photon mismeasurement ratio}

Events with single electrons misidentified as photons are another major source of background. An electron can be misidentified as a photon if the pixel seed in the tracker from the electron's trajectory does not get reconstructed. The main physics process behind this background is the $W \rightarrow e \nu$ production, which has a kinematic signature very similar to the SUSY benchmark model and also have large production cross section.

To estimate the contamination of events due to this misidentification, a control sample enriched with electrons was constructed by reverting the pixel seed veto (PSV) in the photon identification requirements. This control sample was then normalized by the "misidentifcation ratio" that is computed through a tag-and-probe method, analyzing events in which the $Z$ decays to electrons.

Before describing the details of the method, the following definitions must be made:

- $\gamma$ : Objects that pass the complete $e / \gamma$ POG photon ID, including the PSV cut (photon object);

- $\gamma_{e}$ : Objects that pass the photon ID with the PSV cut reversed (misidentified $e$ object); 
- $N_{\gamma_{e}}$ : Number of events in the control sample, which is made of $\gamma_{e}$;

- $\epsilon_{\gamma_{e}}$ : Efficiency of accepting $\gamma_{e}$ objects (including acceptance);

- $\epsilon_{\gamma}$ : Efficiency of accepting $\gamma$ objects (including acceptance);

- $F_{e \rightarrow \gamma}$ : Misidentification rate of the PSV cut for electrons misidentified as photons.

Therefore, the number of electrons misidentified as photons in the signal sample is given by

$$
\begin{aligned}
N_{e \rightarrow \gamma} & =\frac{N_{\gamma_{e}}}{\epsilon_{\gamma_{e}}} \times F_{e \rightarrow \gamma}=N_{\gamma_{e}} \times R \\
R & =\frac{F_{e \rightarrow \gamma}}{\epsilon_{\gamma_{e}}} .
\end{aligned}
$$

This ratio $(R)$ can be related to the PSV misidentification rate because, for the PSV cut, the efficiency and misidentification rate obey the following relation:

$$
\epsilon_{\gamma_{e}}+F_{e \rightarrow \gamma}=1
$$

Therefore,

$$
F_{e \rightarrow \gamma}=\frac{R}{1+R} .
$$

One can reconstruct the number of events in four different cases:

- $Z_{e \gamma}$ : one of the electrons of the $Z$ decay is identified an electron and the other is reconstructed as a photon object;

- $Z_{e \gamma_{e}}$ : one of the electrons of the $Z$ decay is identified as an electron and the other is reconstructed as an misidentified $e$;

- $Z_{\gamma_{e} \gamma}$ : one of the electrons of the $Z$ decay is identified an misidentified $e$ and the other as a photon;

- $Z_{\gamma_{e} \gamma_{e}}$ : both electrons of the $Z$ decay are identified as misidentified es; 
Therefore, the reconstructed number of events in each $Z$ peak case can be represented as:

$$
\begin{aligned}
N_{e \gamma} & =2 \times N^{\prime}(Z \rightarrow e e) \times \epsilon_{e} \times F_{e \rightarrow \gamma} \\
N_{e \gamma_{e}} & =2 \times N^{\prime}(Z \rightarrow e e) \times \epsilon_{e} \times \epsilon_{\gamma_{e}} \\
N_{\gamma \gamma_{e}} & =2 \times N^{\prime}(Z \rightarrow e e) \times F_{e \rightarrow \gamma} \times \epsilon_{\gamma_{e}} \\
N_{\gamma_{e} \gamma_{e}} & =N^{\prime}(Z \rightarrow e e) \times \epsilon_{\gamma_{e}}^{2},
\end{aligned}
$$

where $N_{x y}$ is the number of events of $Z$ decaying to electrons when the electrons are reconstructed as $\mathrm{x}$ and $\mathrm{y}$, and $N^{\prime}(Z \rightarrow e e)$ overall number of expected $Z \rightarrow e e$ in the sample. The factors of two multiplying the first three equations are due to combinatorics.

With that system, we can infer that the calculation of the ratio can be performed in two ways:

$$
R=\frac{F_{e \rightarrow \gamma}}{\epsilon_{\gamma_{e}}}=\frac{N_{e \gamma}}{N_{e \gamma_{e}}}=\frac{1}{2} \frac{N_{\gamma_{e} \gamma}}{N_{\gamma_{e} \gamma_{e}}}
$$

The $Z$ shape was obtained from the template of a DY $\rightarrow e e \mathrm{MC}$ at generator level. This template was then convoluted with a Gaussian to simulate detector resolution effects. The parameters of the Gaussian were then fitted to the obtained invariant mass distribution from the different categories detailed above. For the calculation of $N_{x y}$, the signal function was integrated in the $\pm 2 \sigma$ region to obtain the number of signal events. The fits for the $Z \rightarrow e \gamma$ and $Z \rightarrow e \gamma_{e}$ cases can be seen in Fig. 5.7 .

The background shape for the fit was estimated by the convolution of an error function and a decaying exponential function. The background in the Fig. 5.7 (a) is originated form the combinatorics of the electron plus non-resonant photon-like objects, such as real photons, jets misidentified as photons originating from other interactions. Because the electron is most likely coming from the resonant $\mathrm{Z}$ production, the yield drops at lower values of $M_{e \gamma}$. The small disagreement between the fits and the data around $85 \mathrm{GeV}$ comes from the trigger acceptance. It should be noted that the probe selection $p_{\mathrm{T}}$ for this study is $35 \mathrm{GeV}$.

Assuming a Poissonian uncertainty in the signal integral result, the uncertainties in the ratio and misidentification rate can be estimated as: 

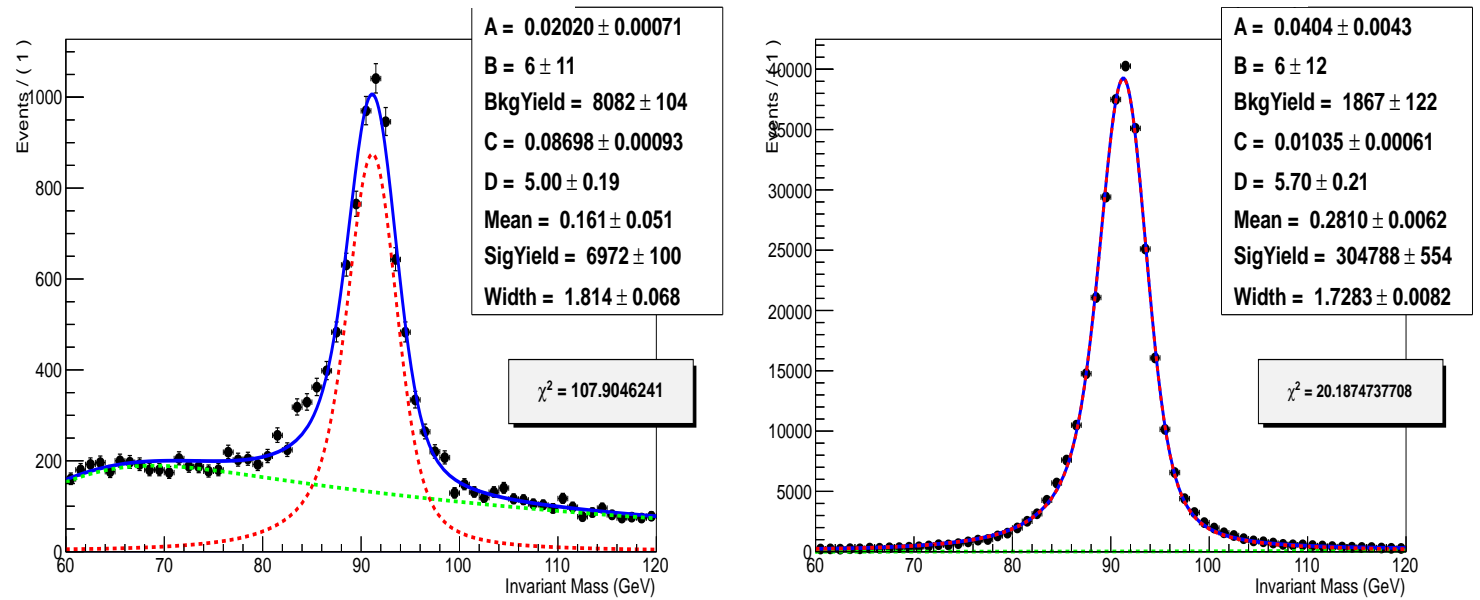

Figure 5.7: Fit of the $Z$ invariant mass for the $Z \rightarrow e \gamma$ case and the $Z \rightarrow e \gamma_{e}$ case.

$$
\begin{aligned}
\sigma_{R} & =\sqrt{\frac{1}{N_{e \gamma_{e}}^{2}} \sigma_{N_{e \gamma}}^{2}+\frac{N_{e \gamma}^{2}}{N_{e \gamma_{e}}^{4}} \sigma_{N_{e \gamma_{e}}}^{2}} ; \\
\sigma_{F R} & =\frac{1}{(1+R)^{2}} \sigma_{R} .
\end{aligned}
$$

With that, we obtain the following results:

$$
\begin{aligned}
R & =(2.38 \pm 0.03) \% ; \\
F_{e \rightarrow \gamma} & =(2.32 \pm 0.03) \%
\end{aligned}
$$

The PSV misidentification rate is dependent on variables such as the probe $p_{T}$, the number of tracks associated with the primary vertex and the number of reconstructed vertices in the event. The nature of the last two dependencies is deeply rooted in the track reconstruction and matching algorithm. To check the dependency of the misidentification rate in the three variables mentioned, the misidentification rate was calculated in exclusive bins. In each bin, the signal template used was the corresponding bin in the MC signal sample. Each fit was done individually for every bin.

In Fig. 5.8, the dependency of the PSV misidentification rate on the probe $p_{T}$, number of tracks associated with the primary vertex and the number of reconstructed primary vertices, respectively is shown. The red line in each plot represents the misidentification rate obtained with the entire invariant mass spectrum. From now on, this first result will be referenced as the "flat misidentification rate". 

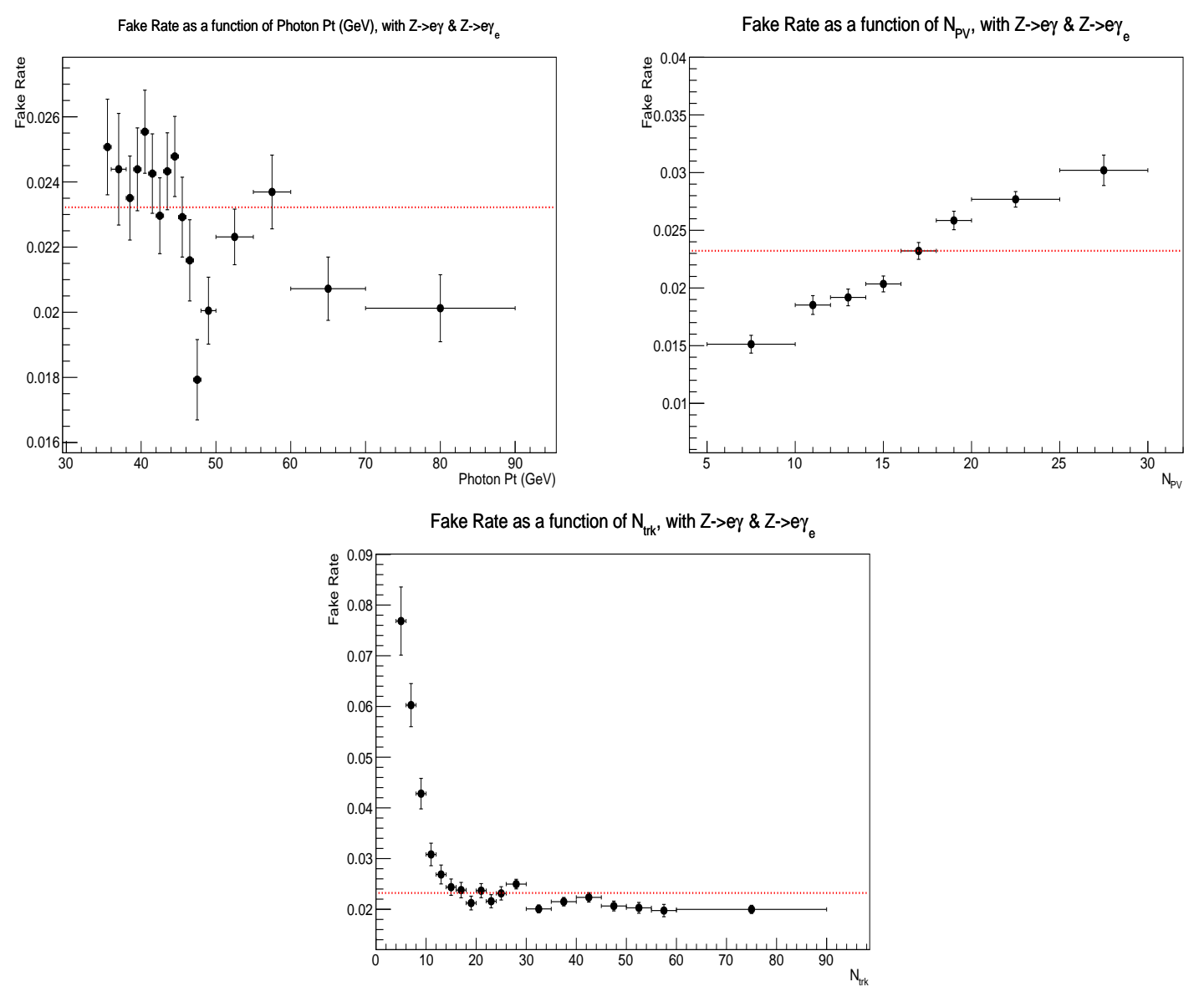

Figure 5.8: Misidentification rate vs. probe $p_{T}$, number of primary vertices and track multiplicity.

As shown in the plots, there is a non-trivial dependency of the misidentification rate on the variables probed. There are different ways to resolve this feature. The most complete one would be to achieve a multi-parameter description of the misidentification rate, including a 3-dimensional function with dependencies of the misidentification rate on each variable. That, however, demands a thorough study of these dependencies and a functional form. A second way would be to choose one of the parametrizations, and prescribe a systematical uncertainty for this measurement.

Figs. 5.9 show the shape and yields of this control sample when normalized by the different parametrization of the misidentification rate. When checking the differences in number of events of each bin, it was observed that the yields were within $5 \%$ of each other, being higher around the $p_{T}$ peak and approaching 0 for higher values. Therefore, choosing any specific parametrization and assigning a systematic uncertainty of $5 \%$ on the number of events would yield in compatible results. For this analysis, flat misidentification ratio has been chosen to estimate the electrons misidentified as photons. 

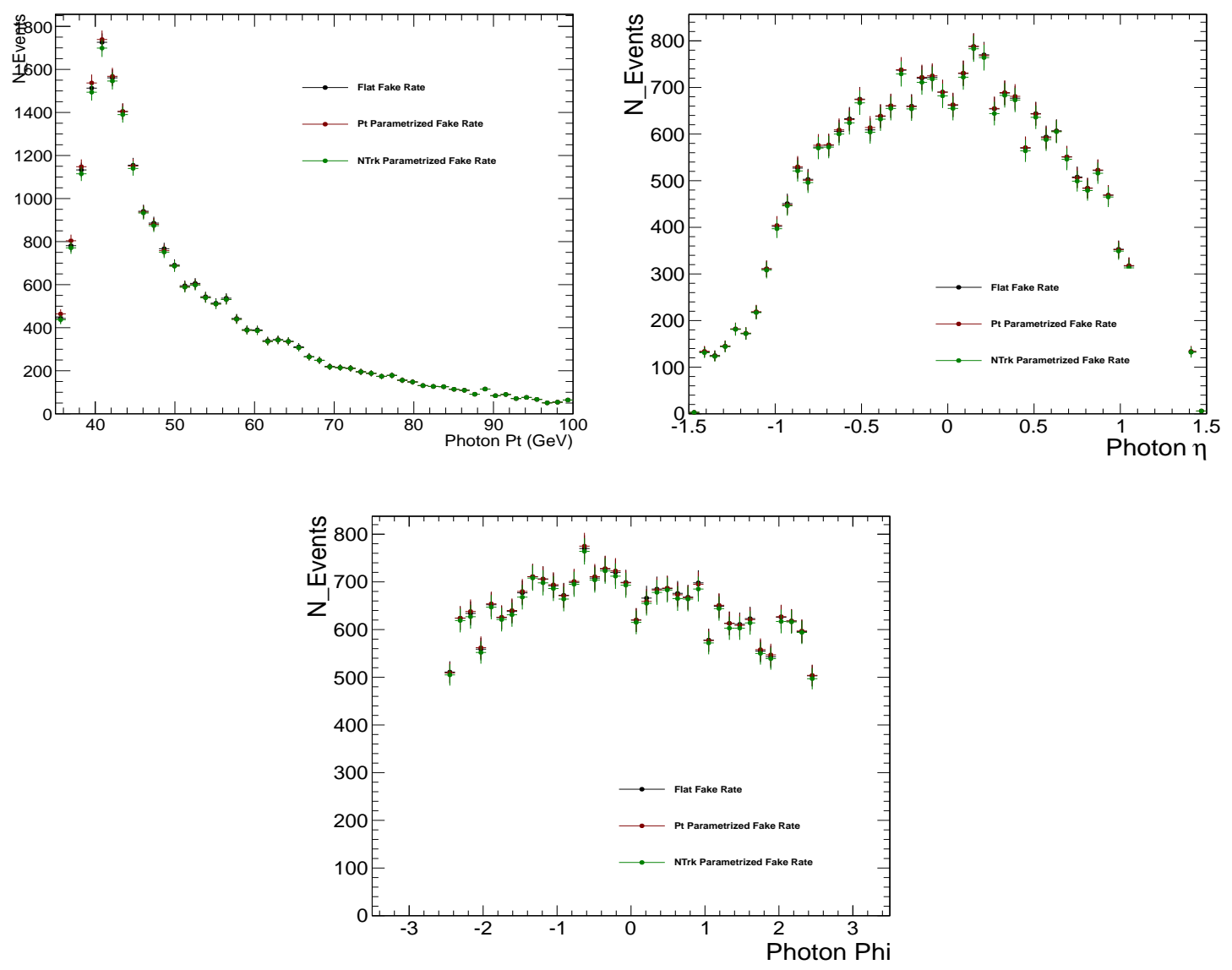

Figure 5.9: Control sample distribution of photon $p_{T}, \eta$, and $\phi$.

To check the robustness of the approach, the misidentification rate has been also computed using the Drell-Yan MC simulation and was compared to the generator-level results. The generator level misidentification rate is defined as:

$$
F_{g e n}=\frac{\text { \#Medium ID Photons \&\& Matched to Gen Electrons \&\& Pass the PSV }}{\text { \#Medium ID Photons \&\& Matched to Gen Electrons }}
$$

Here, the medium working point for the photon identification is the one used on the analysis. The generator level particles used for the generator level misidentification rate to the hardest reconstructed primary vertex with the following selection:

- $D_{z}<0.5$

- $D_{x y}<0.2$. 
Two measurements, generator level and reconstructed object level, are compared and shown in Fig. 5.10. It can be seen that the agreement between the generator level and reconstructed object level misidentification rates are within $4 \%$.

$\mathrm{e} \rightarrow \gamma$ Fake Rate vs. Probe Pt

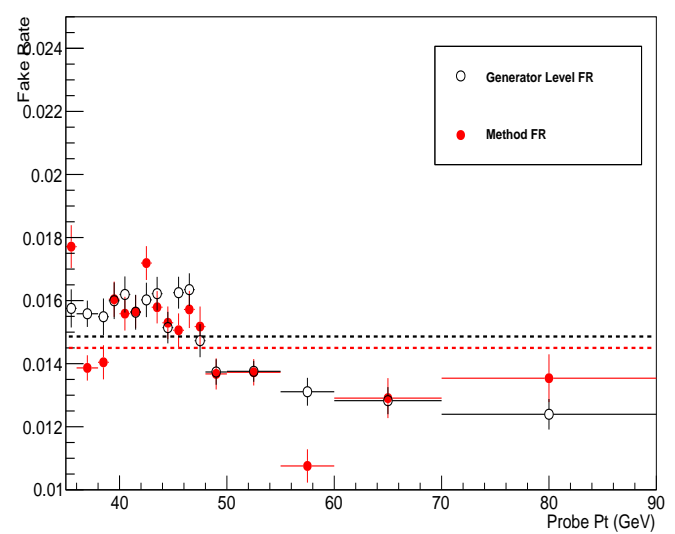

$e \rightarrow \gamma$ Fake Rate vs. Pile Up

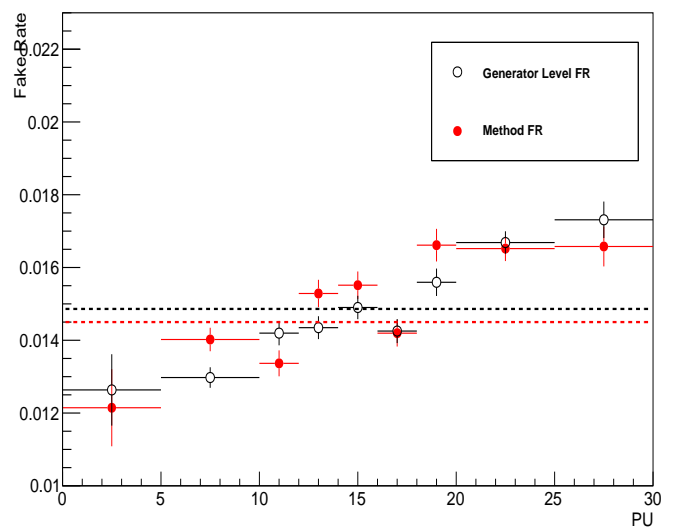

$\mathrm{e} \rightarrow \gamma$ Fake Rate vs. Track Mult. of Selected PV

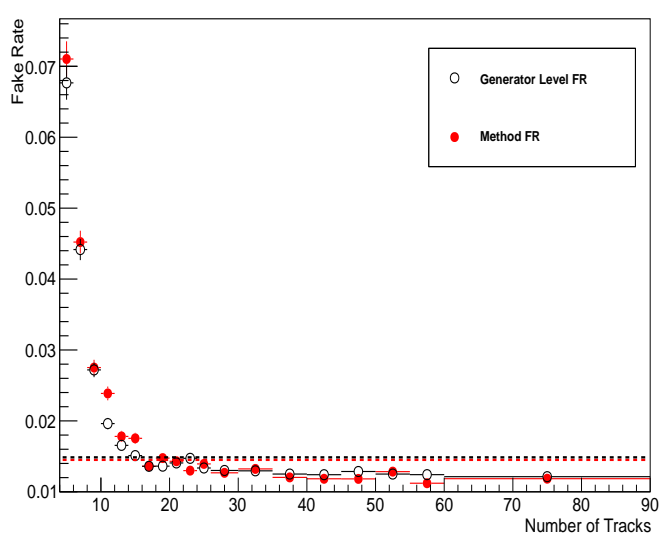

Figure 5.10: PV matched closure test in probe $p_{T}$, number of reconstructeed primary vertices and track multiplicity

Two main systematic uncertainty associated with this method are due to (a) using a flat parameterization (5\%) and (b) closure test, closing the method within $4 \%$. The remaining systematic uncertainty is related to the background estimation Fig. 5.7, i.e. the choice of the functional form to represent the background composition. Two choices were made for that estimation: a simple decaying exponential and the nominal background shape as described before. Looking at the full mass spectrum of the invariant mass, without parametrization, the amount of expected background is much smaller than the number of signal events. The difference in the calculated misidentification rate for the two functions is about $4 \%$. 


\subsubsection{Estimation of jet $\rightarrow$ photon mismeasurement ratio}

Due to the large production cross section QCD multijet events is one of the dominant backgrounds to the single photon analyses. Jets that pass the photon selection are typically enriched in $\pi^{0} \rightarrow \gamma \gamma$ and contain little hadronic activity and are highly collimated. Therefore, the detector response to these jets is similar to that of single photons. To have a robust description of the QCD background, its expected contribution is estimated from data.

The ratio of the jets misidentified as photons are computed using data control sample that is orthogonal to the nominal event selection as a function of $E_{\mathrm{T}}^{\gamma}$. The control sample events are selected by requiring offline photons that are matched to the triggering photon through a $\Delta R<0.3$ selection. These photons are also required to have a minimum energy of $35 \mathrm{GeV}$. The orthogonality of the control data sample to the signal selection is secured by requiring $\not_{T}$ in the event to be less than $40 \mathrm{GeV}$. Potential effect of this selection on the topology of the event (and therefore on the misidentification ratio) is examined in detail and the deviation is assigned as systematic uncertainty for the measurement.

The first step of the measurement is to construct the "raw" misidentification ratio, which is defined as:

$$
F R_{\text {raw }}=\frac{\text { numerator }}{\text { denominator }}
$$

The "numerator" of the raw misidentification ratio is populated by events where photon objects pass the medium identification and isolation criteria. In addition, further shower shape selection such as $\sigma_{i \eta i \eta}>0.001$ and $\sigma_{i \phi i \phi}>0.001$, in order to suppress the contribution of non-collision events.

The "denominator" of the raw misidentification ratio is populated with jets which could be misidentified as photons through requiring loose identification and isolation criteria for photons and further requiring that at least one of the isolation criteria or $\sigma_{i \eta i \eta}$ selection, to have failed. This leads to having an upper and a lower bound on the isolation for each photon. In explicitly, the upper bound denominator selection is:

- $I_{\pi}<\min \left\{5 \times 4.0,0.2 \times E_{\mathrm{T}}^{\gamma}\right\}$;

- $I_{\mathrm{n}}<\min \left\{5 \times\left(4.5+0.04 \times E_{\mathrm{T}}^{\gamma}\right), 0.2 \times E_{\mathrm{T}}^{\gamma}\right\} ;$

- $I_{\gamma}<\min \left\{5 \times\left(4.5+0.005 \times E_{\mathrm{T}}^{\gamma}\right), 0.2 \times E_{\mathrm{T}}^{\gamma}\right\}$.

and the lower bound denominator selection is: 


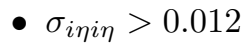

OR $I_{\pi}>4.0$

OR $I_{\mathrm{n}}>4.5+0.04 \times E_{\mathrm{T}}^{\gamma}$

OR $I_{\gamma}>4.5+0.005 \times E_{\mathrm{T}}^{\gamma}$.

Fig. 5.11 shows the raw misidentification ratio as defined for seven different $E_{\mathrm{T}}^{\gamma}$ bins.

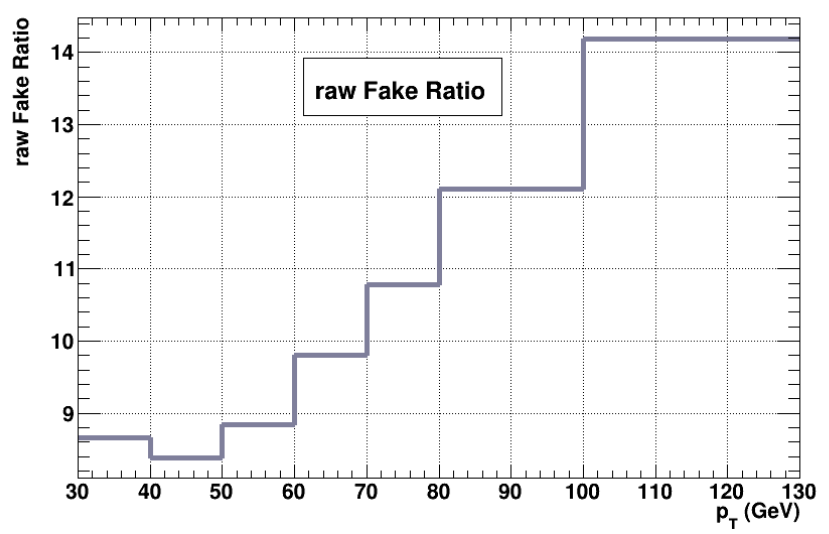

Figure 5.11: Raw misidentification ratio values per photon $E_{\mathrm{T}}$ bin.

The numerator sample will be contaminated by a considerable fraction of true isolated photons from inclusive QCD direct photon production. This contribution needs to be estimated and subtracted to identify the true QCD misidentification ratio through "template method". The "corrected" misidentification ratio, then becomes:

$$
F R_{\text {corrected }}=\frac{\text { numerator }- \text { contamination }}{\text { denominator }} .
$$

The cleaning of the numerator of real photons is done through the template method. The $\sigma_{i \eta i \eta}$ templates are used to distinguish between genuine and misidentified photons in the numerator sample. Mis-identified photon template is constructed by applying numerator-like selection to the data control sample in a sideband region of charged hadron isolation, $2.0 \mathrm{GeV}<I_{\pi}<6.0 \mathrm{GeV}$. The extraction of the templates for the genuine photon shapes is done using MC simulation samples through applying numerator selection requirements and further matching the reconstructed photon object to a generator level object. A $\sigma_{i \eta i \eta}$ correction is applied to real templates in order to have the $\mathrm{MC}$ simulation model data better. The rescaling of $\sigma_{i \eta i \eta}$ is:

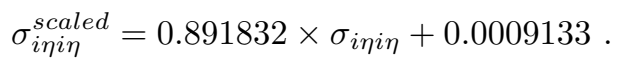


We construct the genuine (MC truth matched), misidentified (data-sideband) and full (datanumerator) $\sigma_{i \eta i \eta}$ templates for seven different photon $E_{\mathrm{T}}$ bins of:

$$
[30-40],[40-50],[50-60],[60-70],[70-80],[80-100],[100-130] \mathrm{GeV}
$$

A likelihood fit using ROOFIT is then performed, of genuine + misidentified templates to full candidate templates per $E_{\mathrm{T}}^{\gamma}$. Figs. 5.12 and 5.13 show the templates per photon $E_{\mathrm{T}}$ bin after performing the fit.
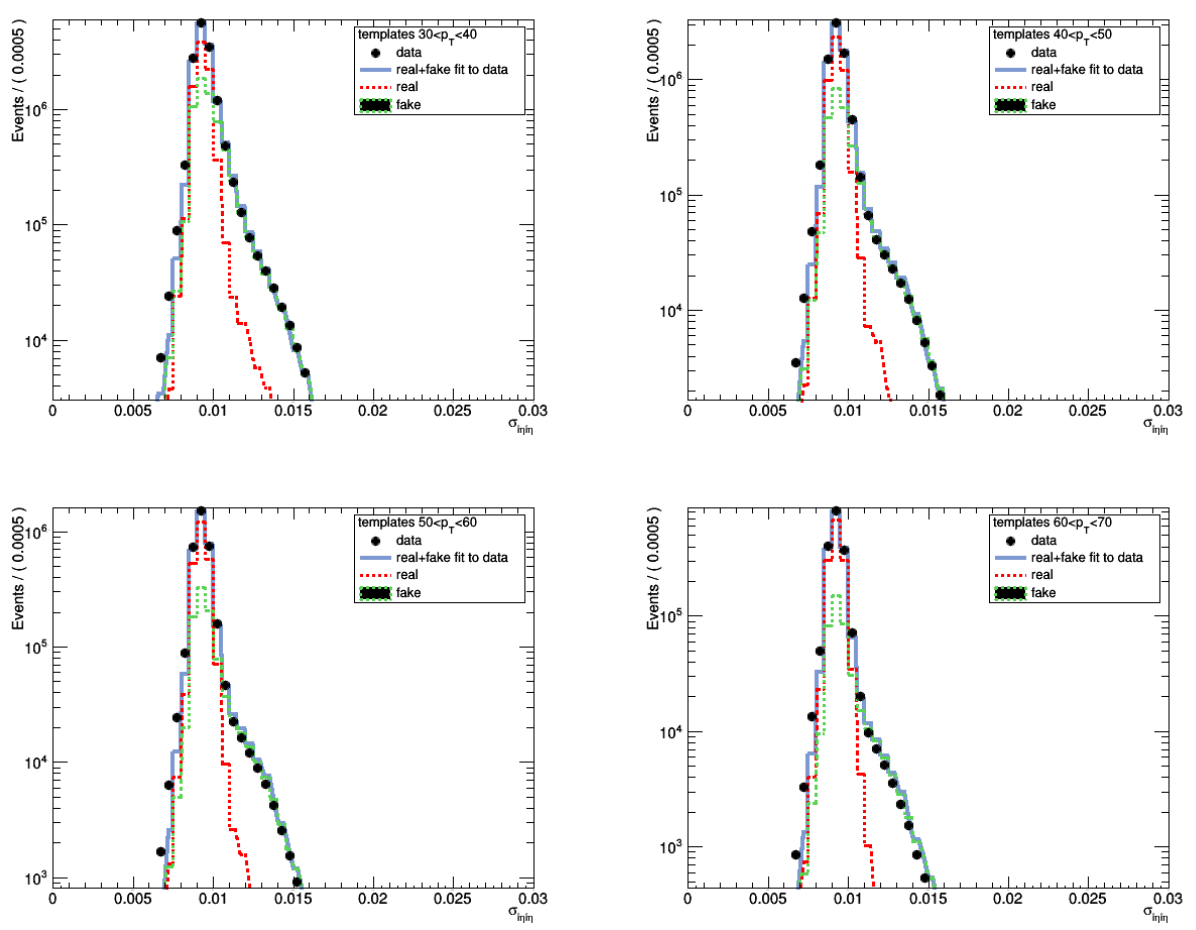

Figure 5.12: $\sigma_{i \eta i \eta}$ templates for different photon $E_{\mathrm{T}}$ bin.

The yields of the genuine photons in the region of $\sigma_{i \eta i \eta}<0.011$ is the contamination and is subtracted from the numerator yields, following equation 5.20. The corrected misidentification ratio is then calculated per photon $E_{\mathrm{T}}$ bin, by simply subtracting the genuine photon contribution from raw numerator. The final fit is performed and the final corrected misidentification ratio as a function of $E_{\mathrm{T}}$ is obtained. The functional form used to parametrize the ratio is;

$$
f_{E_{\mathrm{T}}^{\gamma}}=p 0+\frac{p 1}{\left(E_{\mathrm{T}}^{\gamma}\right)^{22}} .
$$

Fig. 5.14 shows the corrected misidentification ratio as a function of photon $E_{\mathrm{T}}$. It should be 

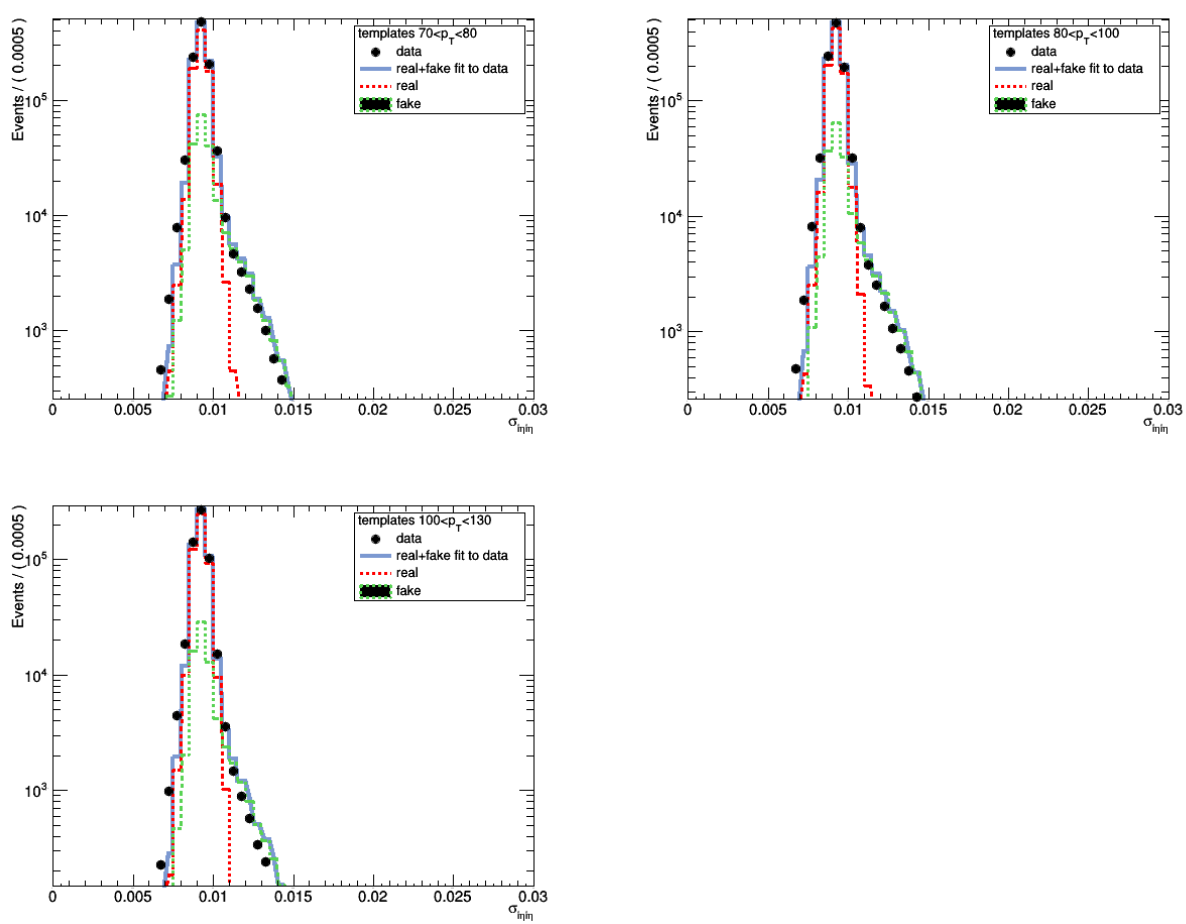

Figure 5.13: $\sigma_{i \eta i \eta}$ templates for different photon $E_{\mathrm{T}}$ bin.

noted that, given the disjoint selection of numerator and denominator samples, these samples are not subset of each other. Therefore, one can expect a ratio $>1$.

In this plot, the x-axis points and errors are calculated by looking at the mean and root mean square of the photon $E_{\mathrm{T}}$ distribution in each $E_{\mathrm{T}}$ bin. The errors on the y-axis are calculated through error propagation based on the fit errors and are very small. Finally, the QCD yield is estimated by applying the above function to the reconstructed photons of the analysis sample, which pass the nominal selection, but have the photon selection replaced by denominator selection.

One of the sources of systematic uncertainty in this procedure is estimated by examining the denominator selection definition. Through varying the lower bound definition in the denominator selection, new misidentification ratio functions were obtained. In explicitly we the following selection was imposed:

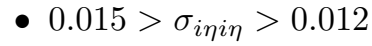

OR $I_{\pi}>4.0$

OR $I_{\pi}>4.5+0.04 \times E_{\mathrm{T}}^{\gamma}$,

where the cases differ by having no selection on the $I_{\gamma}$ and $I_{\mathrm{n}}$. 


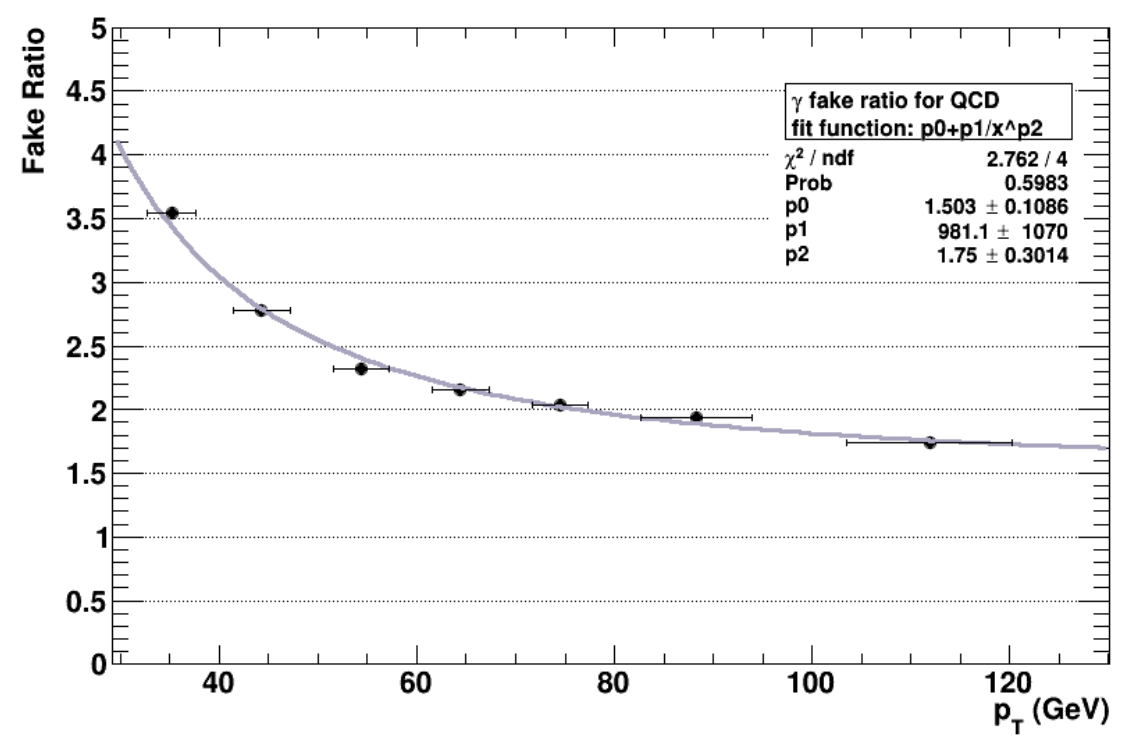

Figure 5.14: Final corrected misidentification ratio as a function of photon $E_{\mathrm{T}}$.

This procedure leads to a different denominator and as a result a different misidentification ratio as shown in Fig. 5.15 However as mentioned above what is to be compared is the corresponding yield in the analysis data for denominator like objects, therefore different denominator leads to different yields. These final yields are plotted in Fig. 5.15 for the different denominator cases and their difference is found to be negligible.
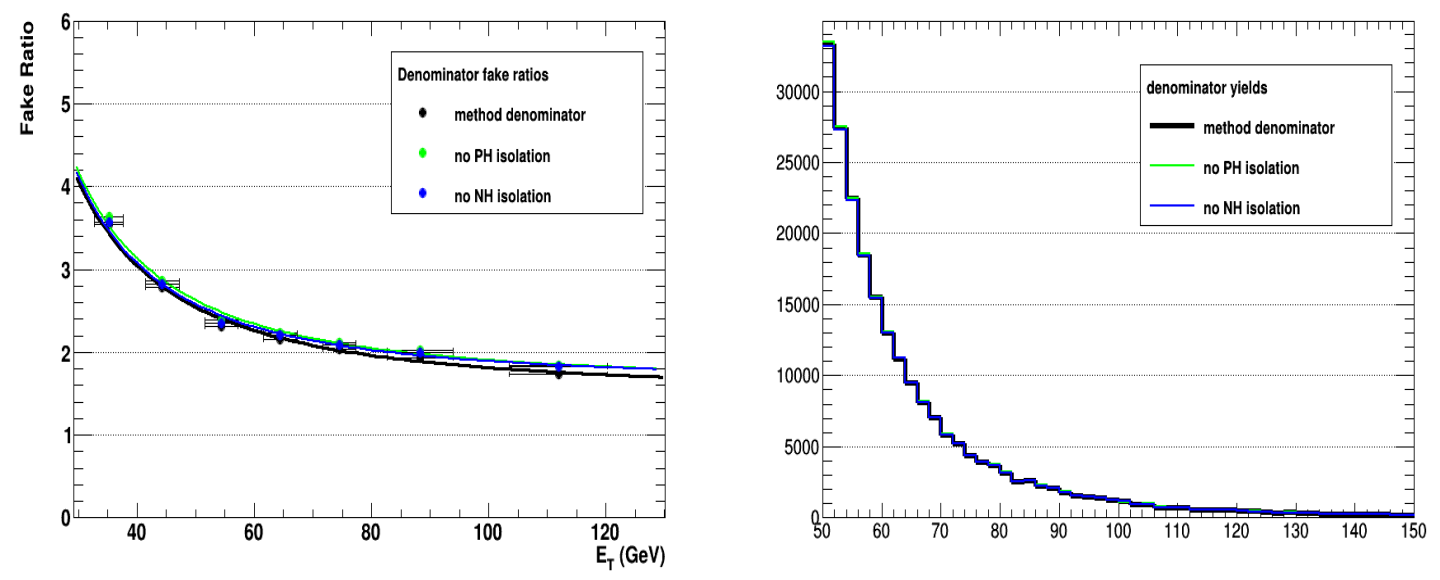

Figure 5.15: Corrected misidentification ratio as a function of photon $E_{\mathrm{T}}$ for different denominator definitions and the final corresponding yields in the analysis data.

An other source of systematic uncertainty is due to the variation in the $\sigma_{i \eta i \eta}$ template binning. 
Various template binning sizes were examined and found to have negligible contribution. In particular the misidentification ratio by creating templates with half and double bin size to estimate the photon contamination is recalculated. The effect of the new binning on the misidentification ratio can be seen in Fig. 5.16

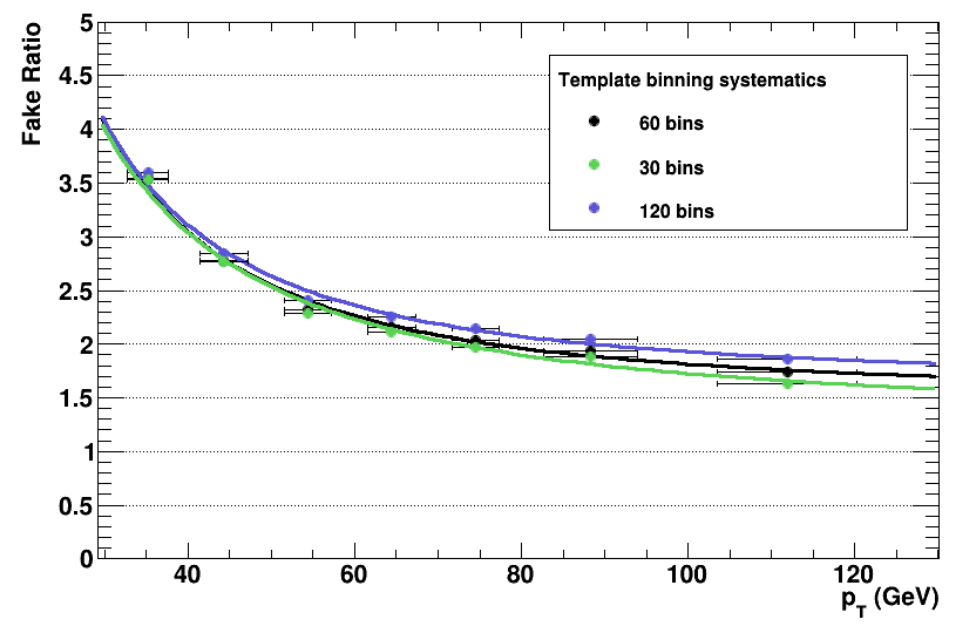

Figure 5.16: Corrected misidentification ratio as a function of photon $E_{\mathrm{T}}$ for different template binning.

An other source of systematic uncertainty is due to the $\not_{T}$ selection when constructing the control sample. To evaluate the dependency of this selection on the final misidentification ratio, the misidentification ratio has been calculated as a function of $E_{\mathrm{T}}^{\gamma}$ for various $\not_{T}$ selection as shown on Fig. 5.17.
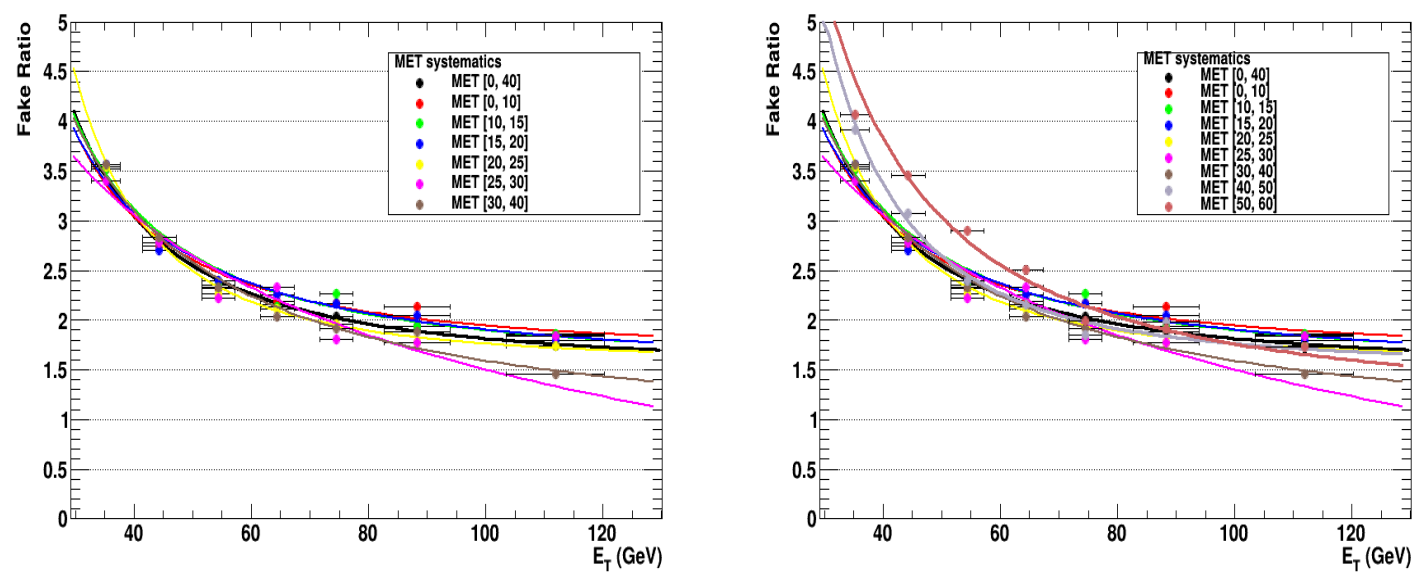

Figure 5.17: Corrected misidentification ratio as a function of photon $E_{\mathrm{T}}$ for different $\not_{T}$ regions. 
Finally to compute the systematic uncertainty due to the choice of the sideband selection for the misidentified photons template the misidentification ratio has been computed using different the sideband selection, closer to the "genuine photon" region and closer to the"misidentified photon" region. Fig. 5.18 shows the corresponding misidentification ratios.

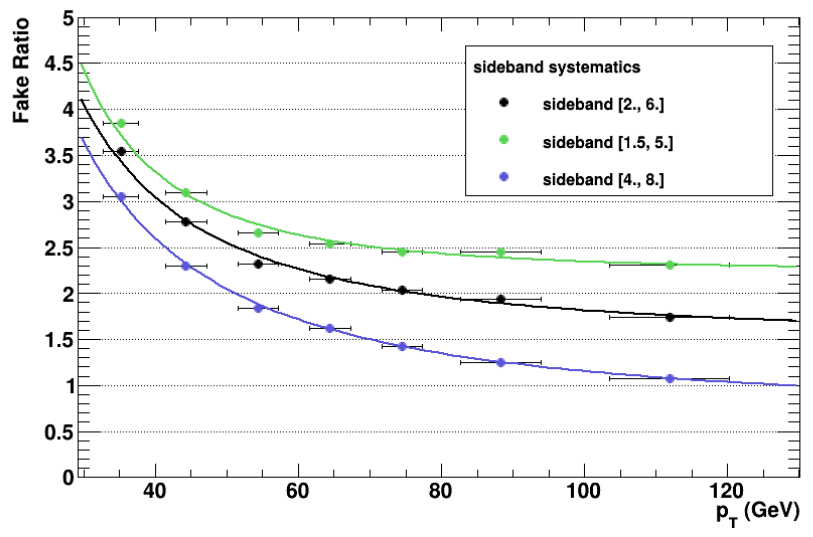

Figure 5.18: Corrected misidentification ratio as a function of photon $E_{\mathrm{T}}$ for different sideband definitions.

As can be seen, the systematic uncertainty associated with the sideband selection for the template method is proven to be the dominant source with $35 \%$.

To check the validity of the method, closure tests were performed. The ratio was calculated using reconstructed and generator level objects in the QCD MC simulation sample. The MC truth ratio was constructed by using generated particle information and constructing the MC truth numerator and denominator, and again subtracting all genuine generated photons. The ratio is shown for both cases in Fig. 5.19.

The comparison of the two approach shows that method is well constructed.

\subsubsection{Non-Collision Backgrounds}

The search is susceptible to contamination from non-collision backgrounds which arise from cosmic ray interactions, spurious signals in the ECAL (spikes), and accelerator induced secondary particles (beam halo). These backgrounds have different arrival time distributions compared to prompt photons produced in hard scattering. To quantify the contamination due to these backgrounds a fit is performed to the candidate time distribution using background templates derived from the data. The various templates are formed in the following way: 


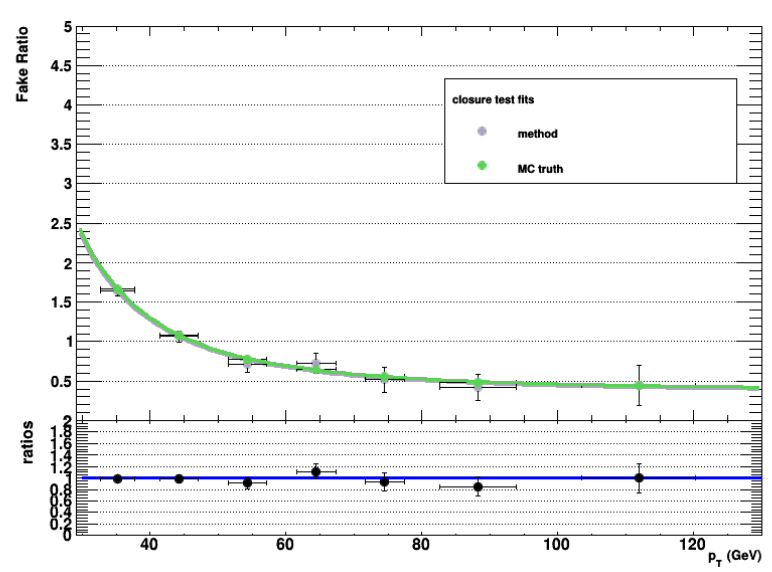

Figure 5.19: Closure test on the method. Misidentification ratio method and MC truth misidentification ratio fits comparison and their ratio, using QCD MC simulation.

- Candidate sample templates: To construct the template for the candidate sample, requirements similar to the analysis selection are applied with relaxed the shower shape variables and without offline $R_{9}$ selection. No mismeasured $\not_{T}$ rejection selection was applied to increase the statistics in the templates. The distribution can be seen in Fig. 5.20 (a).

- Prompt templates: The templates for prompt events is constructed using the candidate sample in which the photon candidates are required to have a pixel seed match, as seen in Fig. 5.20 (b).

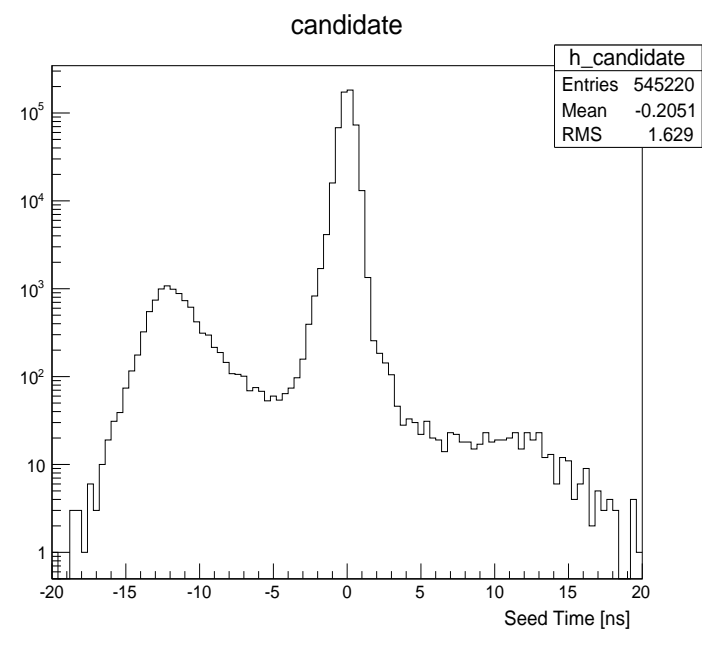

(a) candidate sample

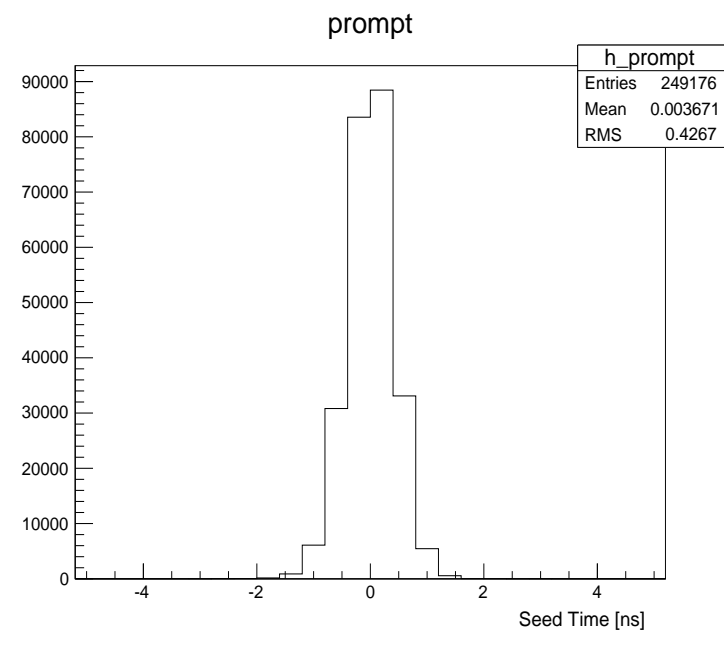

(b) with pixel seed match

Figure 5.20: Seed time distribution (ns) for candidate events and prompt events. 
- Spikes templates: The spike template is constructed by reversing one of the shower shape variables. These templates are shown in Fig. 5.21 (a).

- Beam Halo templates: The energy deposition from the beam halo muon could be reconstructed within the prompt window when the muon brems in the ECAL. To get the template of timing distributions of such objects, similar selection used for the candidate sample was applied but further required the minimum ionizing particle total energy (MIP) to be greater than $4 \mathrm{GeV}$. The beam halo template is shown in Fig. 5.21 (b). The MIP-tagging is a set of criteria developed in order to identify the passage of a beam halo muon across the acceptance of the ECAL. Crystals other than associated with electromagnetic shower are identified along potential paths in a similar $\phi$ direction as of the seed crystal of EM shower, and then fitted with a straight line. The end results is the number of crystals associated along the selected trajectory, their total energy (MIP energy), and the $\chi^{2}$ of the fitted line. The beam hallo shower typically have more MIP energy than a true EM shower .

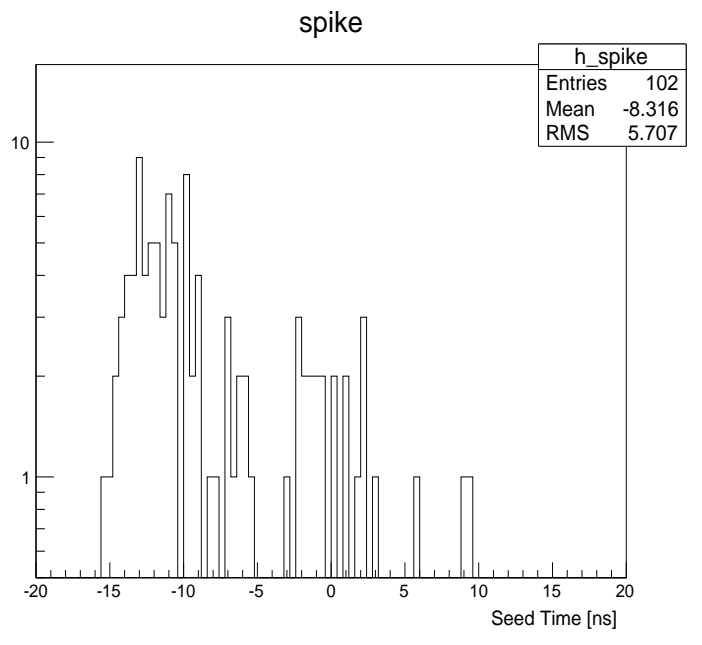

(a) with unphysically small shower shape variables

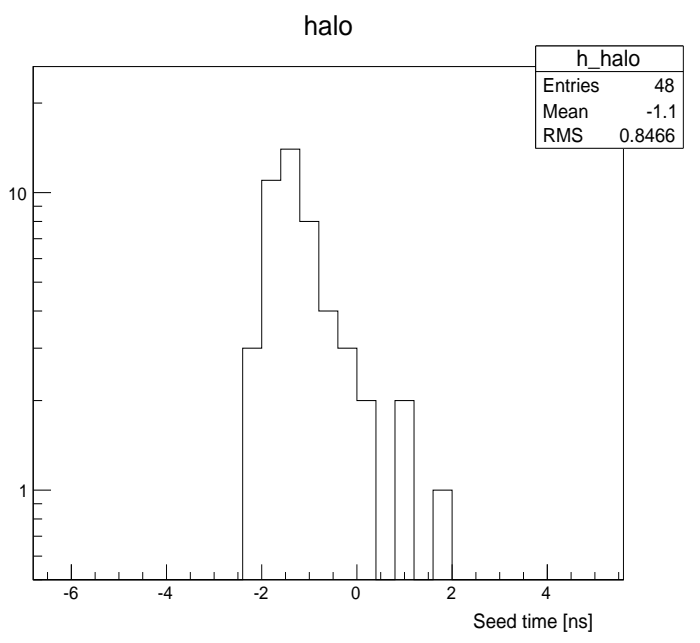

(b) MIP Tagged events

Figure 5.21: Seed time distribution (ns) for spike and halo events

The final number of each non-collision background events in data are estimated by the fitting the template distributions of spike, beam halo and prompt photons to the complete timing distribution of the candidate events. Through this, it was observed that the spike hypothesis is completely rejected, where the beam halo contribution is on the order of $<1 \%$ of the total expected events.

In conclusion, the contamination due to out-of-time background contribution is found to be negligible, therefore not included in the final event yield. 

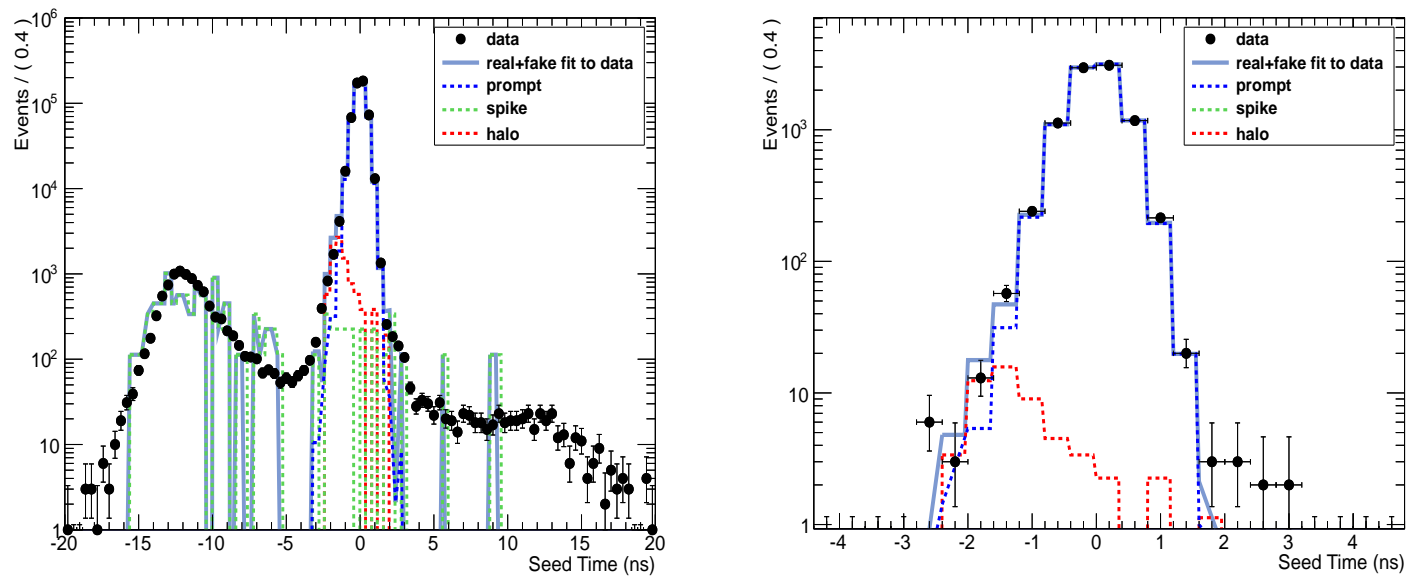

Figure 5.22: Template fits to the candidate selection and model independent analysis selection.

\subsection{Background Modeling Validation}

The background modeling is examined in several control regions. A control sample enriched in $W(l \nu) \gamma$ events is defined with inverted lepton-veto requirement in the preselection, thus selecting events with a loose $e$ or $\mu$. It is expected to be free of any signal contamination due to the presence of a lepton. Another control sample enriched in $\gamma+$ jet events is constructed by just requiring no selection other than the preselection requirements. Fig. 5.23 shows the data vs SM expectation in the two control regions. The observed data and estimated SM backgrounds are found to be consistent both in yield and shape.

Table 5.4: Event yields for data vs. SM expectation in a control region enriched by (a) $\gamma+$ jet events, and $(\mathrm{b}) \mathrm{W}(l \nu)$ events.

\begin{tabular}{|c|c|}
\hline Process & Estimate \\
\hline$\gamma+$ jets & $(1012 \pm 162) \times 10^{3}$ \\
jet $\rightarrow \gamma$ & $(344 \pm 120) \times 10^{3}$ \\
$\mathrm{e} \rightarrow \gamma$ & $(171 \pm 10) \times 10^{2}$ \\
$W(\rightarrow \ell \nu)+\gamma$ & $4242 \pm 212$ \\
$Z(\rightarrow \nu \bar{\nu})+\gamma$ & $2457 \pm 122$ \\
Other & $27124 \pm 44$ \\
\hline Total background & $(1383 \pm 284) \times 10^{3}$ \\
\hline Data & $1386 \times 10^{3}$ \\
\hline
\end{tabular}

\begin{tabular}{|c|c|}
\hline Process & Estimate \\
\hline$\gamma+$ jets & $1994 \pm 319$ \\
jet $\rightarrow \gamma$ & $1591 \pm 557$ \\
$\mathrm{e} \rightarrow \gamma$ & $765 \pm 45$ \\
$W(\rightarrow \ell \nu)+\gamma$ & $2636 \pm 131$ \\
$Z(\rightarrow l \bar{l})+\gamma$ & $267 \pm 16$ \\
Other & $1696 \pm 83$ \\
\hline Total background & $8953 \pm 1279$ \\
\hline Data & 8247 \\
\hline
\end{tabular}

Although the observed data and estimated SM backgrounds are found to be consistent in yield and in shape in majority of the distributions, due to possible mismodeling of the $\gamma+$ jets background, the $\Delta \phi\left(\not_{T}, \gamma\right)$ distribution showed a shape discrepancy for the control region enriched with $\gamma+$ jets . To account for this discrepancy, a re-weighting of the $\gamma+$ jet background with respect to the data 

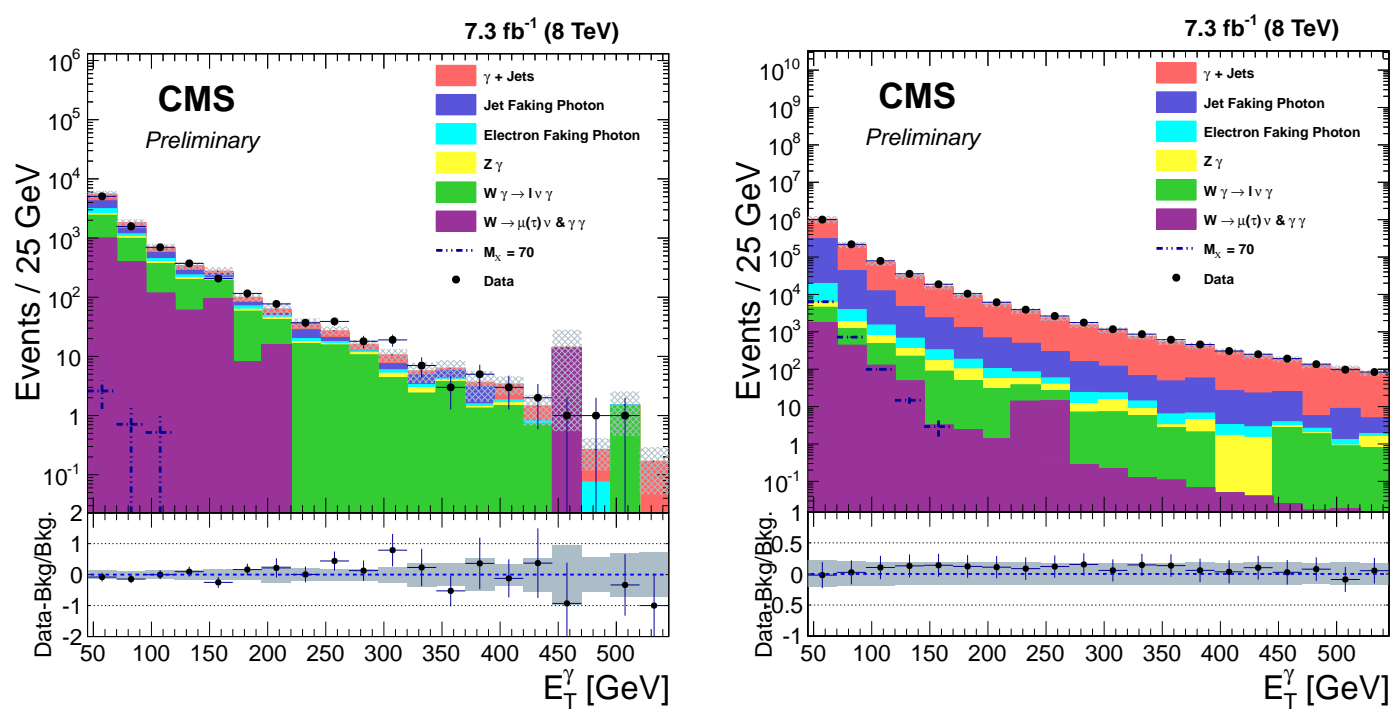

Figure 5.23: The $E_{\mathrm{T}}^{\gamma}$ distributions for data vs. SM expectation in a control region enriched by (a) $\mathrm{W}(l \nu)$ events, and (b) $\gamma+$ jet events. The bottom panels in each plot show the ratio of (data background)/background and the gray band includes both the statistical and systematic uncertainty in the background prediction.

shape in this region was performed. Through this re-weighting, the modulation in the transverse mass distribution also disappears. Both $\Delta \phi\left(\not_{T}, \gamma\right)$ and the transverse mass distributions can be seen in Figs. 5.24 and 5.25 .
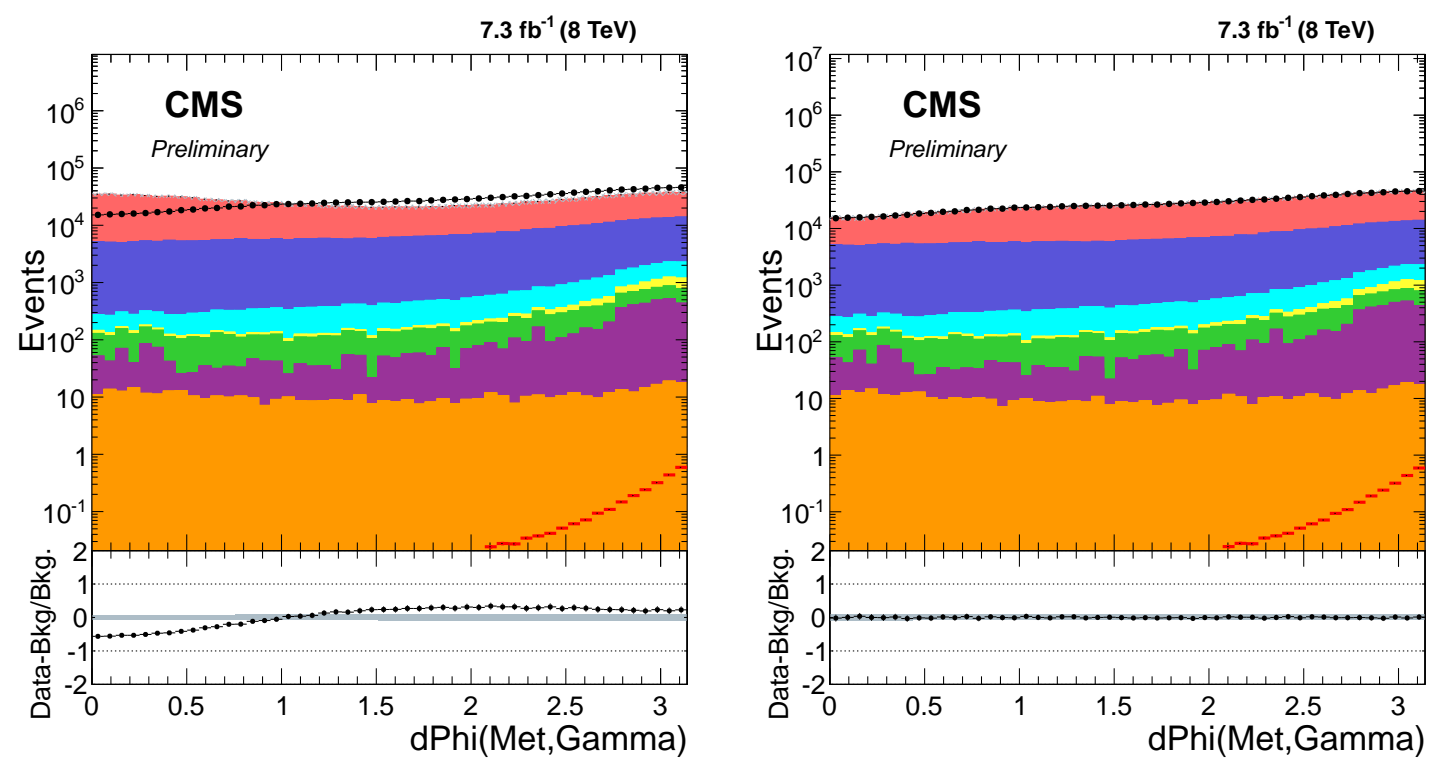

Figure 5.24: Data vs background prediction comparison for the $\Delta \Phi\left(\gamma, \not_{T}\right)$ before and after the reweighting of the $\gamma+$ jet background.

Lastly, the NJet and HT distributions for the pre-selection level after all the corrections that 
have been shown in Fig. 5.26

\subsection{Systematic Uncertainties}

The experimental systematic uncertainties considered in the analysis are listed in Table 5.5. Since the model independent and model specific selections differ significantly, e.g. the inclusion of the mismeasured $\not_{T}$ reduction selection and $E_{\mathrm{T}}^{\gamma}$ window for the model specific results, the systematic uncertainties are evaluated separately for each selection. The systematic uncertainty associated with the measurement of the integrated luminosity is $2.6 \%$ 91. The photon energy scale uncertainty 92 of about $1.0 \%$ affects the signal and background predictions by $4 \%$ for the model specific selection and by $0.5 \%$ for the model independent selection. Similarly, the jet energy scale uncertainty affects the signal and background predictions by $2-5 \%$ depending on the process and selection. When varying the photon or jet energy scales, the $\not_{T}$ is also recomputed. In addition, the systematic uncertainty associated with the jet energy resolution(0.5\%) and unclustered energy (energy not contained within jets or leptons or photons) scale(2\%) are propagated to the $\not_{T}$ and effect the signal and background predictions by $2-4 \%$. The uncertainty due to the choice of PDFs is estimated following PDF4LHC recommendations $90,93,94$ and are found to be $10 \%$ for SUSY Higgs signal, and $4 \%$ for $Z \nu \nu \gamma$ and the $W \gamma$. An additional uncertainty due to the choice of renormalization and factorization scales is evaluated using MCFM and is found to be $3 \%$ for $Z \nu \nu \gamma$ and the $W \gamma$. As described in the previous section, a $16 \%$ uncertainty is applied to the $\gamma+$ jet normalization due to the difference in the jet multiplicity distribution between the data and background prediction in the $\gamma+$ jet control region. The uncertainty due to the pileup modeling is found to be $1 \%$ and is estimated by shifting the central value of the total inelastic cross section from $69.4 \mathrm{mb}$ to $73.5 \mathrm{mb}$. Finally, the systematic uncertainties associated with backgrounds estimated from data have been applied as discussed in previous section.

\subsection{Results}

To evaluate $95 \%$ confidence level (CL) limits on the new physics production cross section, an asymptotic $\mathrm{CL}_{S}$ method 95,96 is used where the systematic uncertainties on the signal and background predictions are treated as nuisance parameters with log-normal prior distributions. 

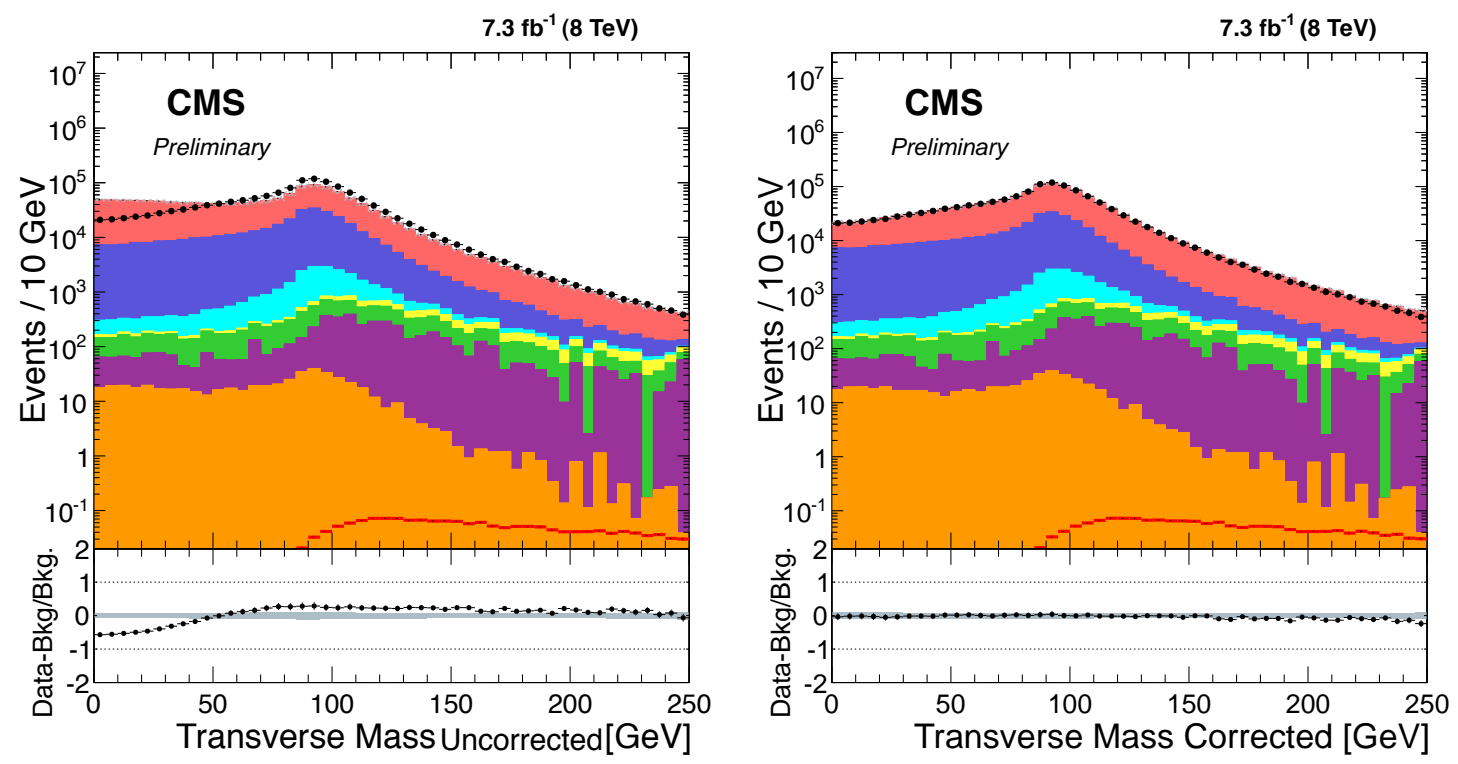

Figure 5.25: Data vs background prediction comparison for the MT distribution before and after the reweighting of the $\gamma+$ jet background for $\Delta \Phi\left(\gamma, \not_{T}\right)$.
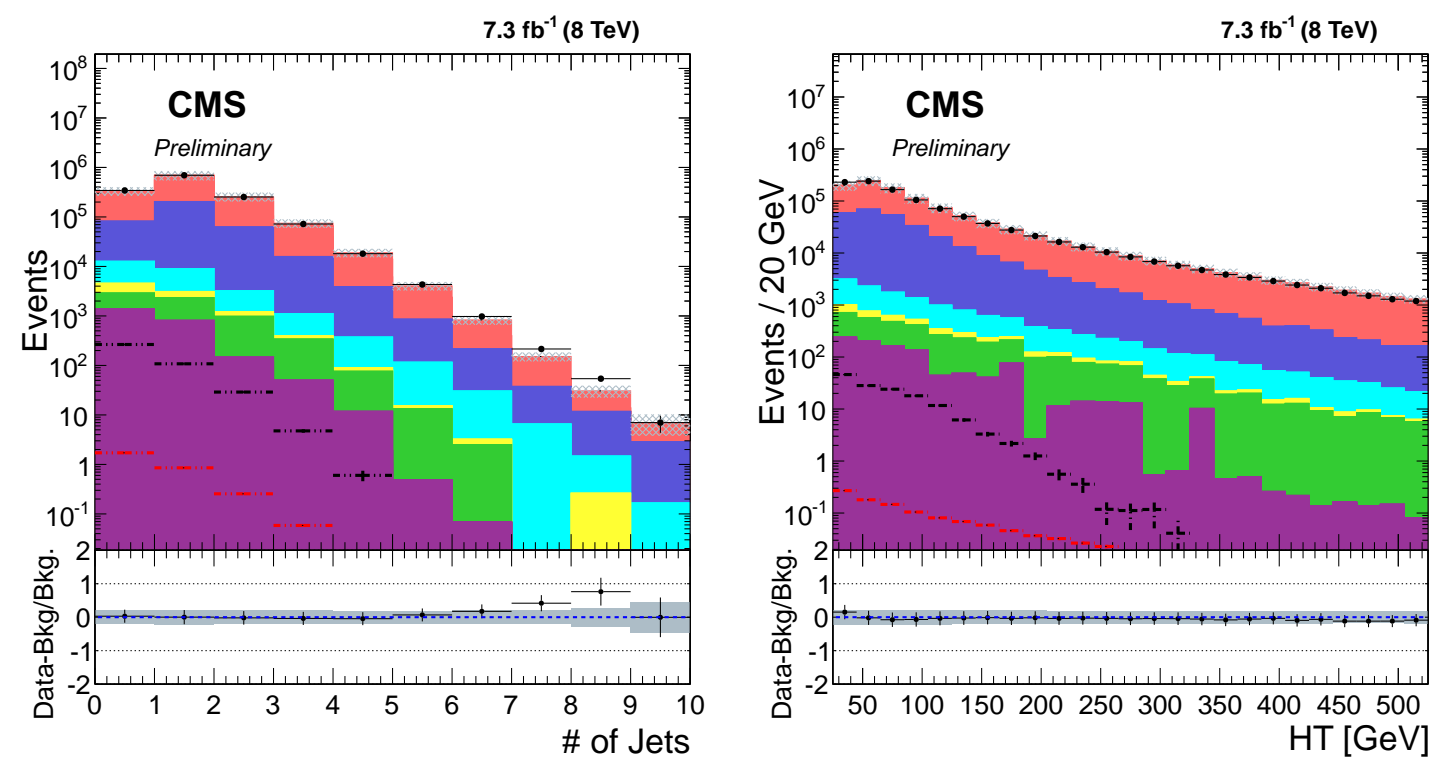

Figure 5.26: Number of Jet (left) and HT (right) distributions in the $\gamma+$ Jet control Region. 
Table 5.5: Summary of all systematic relative uncertainties in percent on $A \times \epsilon_{M C}$ calculation for SUSY Higgs model (Model Independent) selection.

\begin{tabular}{|c|c|c|c|c|c|c|}
\hline Source & Signal & Jet $\rightarrow \gamma$ & Electron $\rightarrow \gamma$ & $\gamma+$ jet & $Z \nu \nu \gamma$ & $W \gamma$ \\
\hline PDF & $10(0)$ & - & - & - & $4(4)$ & $4(4)$ \\
\hline Luminosity & $2.6(2.6)$ & - & - & $2.6(2.6)$ & $2.6(2.6)$ & $2.6(2.6)$ \\
\hline Photon Energy Scale $\pm 1 \%$ & $4(0.5)$ & - & - & $4(0.5)$ & $4(0.5)$ & $4(0.5)$ \\
\hline$\not_{T}$ Energy Scale & $4(2)$ & - & - & $4(2)$ & $4(2)$ & $4(2)$ \\
\hline Jet Energy Scale & $3(2)$ & - & - & $5(5)$ & $3(2)$ & $3(2)$ \\
\hline Pileup & $1(1)$ & - & - & $1(1)$ & $1(1)$ & $1(1)$ \\
\hline$Z \nu \nu \gamma$ MCFM NLO Calculation & - & - & - & - & $3(3)$ & - \\
\hline$\gamma+$ jet normalization & - & - & - & $16(16)$ & - & - \\
\hline$W \gamma$ MCFM NLO Calculation & - & - & - & - & - & $3(3)$ \\
\hline Jet $\rightarrow \gamma$ Unc. & - & $35(35)$ & - & - & - & - \\
\hline Electron $\rightarrow \gamma$ Unc. & - & - & $6(6)$ & - & - & - \\
\hline
\end{tabular}

\subsubsection{Model-Independent Limits}

Due to the variety of signals which can contribute to this final state, we present results for a generic signal using the model-independent selection described in Section 5.2. Although this selection does not have as strong of discrimination power between signal like and background like events compared to the misreconstructed $\not \phi_{T}$ rejection selections, it does have less model dependence. This is due to $\not_{T}$ significance and $\tilde{\mathscr{E}}_{T}$ minimization requirements having a non trivial efficiency dependence on the underlying event and observed $\not_{T}$.

The total expected SM background and observed data events after the model-independent selection are found to be compatible within the systematic uncertainties. Table 5.6 shows a comparison of the event yields estimated for background processes and the observed data. Fig. 5.27 shows the $M_{T}$ and $\not E_{T}$ distributions after the model-independent selection has been applied.

Table 5.6: Comparison of event yields for observed data and background, after the modelindependent selection.

\begin{tabular}{|c|c|}
\hline Process & Event Yields \\
\hline$\gamma+$ jets & $(313 \pm 50) \times 10^{3}$ \\
jet $\rightarrow \gamma$ & $(906 \pm 317) \times 10^{2}$ \\
$\mathrm{e} \rightarrow \gamma$ & $(1035 \pm 62) \times 10^{1}$ \\
$W(\rightarrow \ell \nu)+\gamma$ & $2239 \pm 111$ \\
$Z(\rightarrow \nu \bar{\nu})+\gamma$ & $2050 \pm 102$ \\
Other & $1809 \pm 91$ \\
\hline Total background & $(420 \pm 82) \times 10^{3}$ \\
\hline Data & $442 \times 10^{3}$ \\
\hline
\end{tabular}

Fig. 5.28 shows the observed and expected model-independent 95\% CL upper limits on $\sigma \times B R \times$ $A \times \epsilon$ for different $\not \not_{T}$ and $M_{T}$ thresholds. The observed and expected limits are also shown in Fig 5.28 (c) at a $95 \% \mathrm{CL}$ for $M_{T}>100 \mathrm{GeV}$ and as a function of $\not_{T}$. 

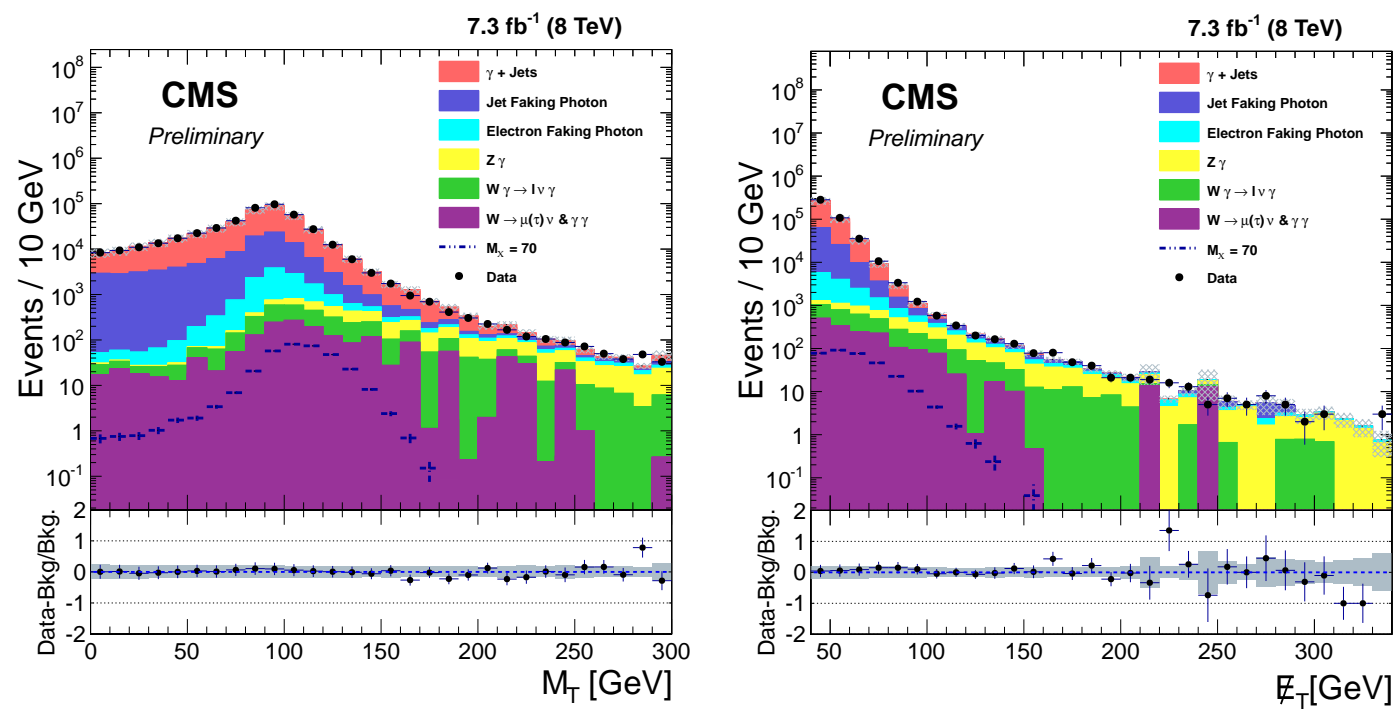

Figure 5.27: The $M_{T}$ and $\not_{T}$ distributions for data, background estimates, and signal after the model-independent selection. The bottom panels in each plot show the ratio of (data - background)/background and the gray band includes both the statistical and systematic uncertainty on the background prediction.
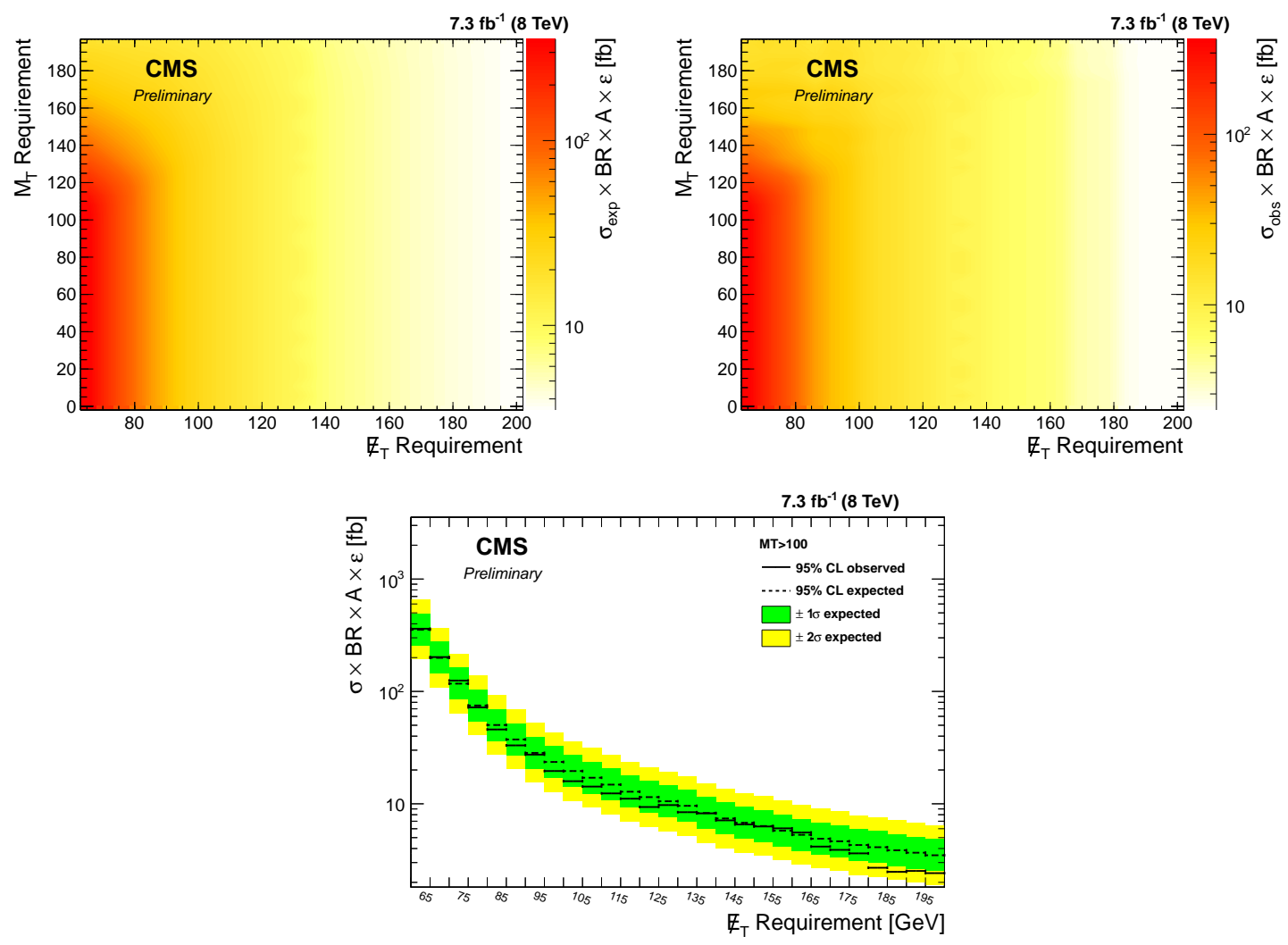

Figure 5.28: The expected (a) and observed (b) 95\% CL upper limit on $\sigma \times B R \times A \times \epsilon$ for different $M_{T}$ and $\not_{T}$ thresholds and (c) for $M_{T}>100 \mathrm{GeV}$ as function of the $\not_{T}$ threshold. 


\subsubsection{Model-Specific Limits}

The yields for supersymmetric decays of the Higgs boson $\left(h \rightarrow \widetilde{G} \widetilde{\chi}_{1}^{0}, \widetilde{\chi}_{1}^{0} \rightarrow \widetilde{G} \gamma\right)$ are acquired through imposing the model-specific selection described in Section 5.2. The yields for this selection are shown in Table 5.7. When considering heavier Higgs boson masses, the maximum $E_{\mathrm{T}}^{\gamma}$ requirement is removed, and the minimum $E_{\mathrm{T}}^{\gamma}$ requirement is chosen to optimize the sensitivity for each mass hypothesis. The yields for the signal selection are shown in Table 5.8 .

Table 5.7: Expected (SM background) and observed event yields after the selection optimized for the supersymmetric decay of the Higgs boson $\left(h \rightarrow \widetilde{G} \widetilde{\chi}_{1}^{0}, \widetilde{\chi}_{1}^{0} \rightarrow \widetilde{G} \gamma\right)$.

\begin{tabular}{|c|c|}
\hline Process & Event Yields \\
\hline$\gamma+$ jets & $179 \pm 28$ \\
jet $\rightarrow \gamma$ & $269 \pm 94$ \\
$\mathrm{e} \rightarrow \gamma$ & $355 \pm 28$ \\
$W(\rightarrow \ell \nu)+\gamma$ & $154 \pm 15$ \\
$Z(\rightarrow \nu \bar{\nu})+\gamma$ & $182 \pm 13$ \\
Other & $91 \pm 10$ \\
\hline Total background & $1232 \pm 188$ \\
\hline Data & 1296 \\
\hline
\end{tabular}

Table 5.8: Signal predictions at $8 \mathrm{TeV}$ in gluon-fusion channel. The signal predictions correspond to $\mathrm{BR}(\mathrm{H} \rightarrow$ invisible $+\gamma)=100 \%$ assuming the SM $\mathrm{H}$ cross section at the given $m_{\mathrm{H}}$ hypothesis. Statistical and systematic uncertainties are quoted.

\begin{tabular}{|c|c|}
\hline Process & Yields \\
\hline \hline$m_{\mathrm{H}}=125 \mathrm{GeV}, m_{\chi_{1}^{0}}=65 \mathrm{GeV}$ & $653.0 \pm 77$ \\
$m_{\mathrm{H}}=125 \mathrm{GeV}, m_{\chi_{1}^{0}}=95 \mathrm{GeV}$ & $1158.1 \pm 137$ \\
$m_{\mathrm{H}}=125 \mathrm{GeV}, m_{\chi_{1}^{0}}=120 \mathrm{GeV}$ & $2935.0 \pm 349$ \\
\hline$m_{\mathrm{H}}=125 \mathrm{GeV}, m_{\chi_{1}^{0}}=95 \mathrm{GeV} \tau_{\chi_{1}^{0}}=100 \mathrm{~mm}$ & $983.2 \pm 112$ \\
$m_{\mathrm{H}}=125 \mathrm{GeV}, m_{\chi_{1}^{0}}=95 \mathrm{GeV} \tau_{\chi_{1}^{0}}=1000 \mathrm{~mm}$ & $463.4 \pm 55$ \\
$m_{\mathrm{H}}=125 \mathrm{GeV}, m_{\chi_{1}^{0}}=95 \mathrm{GeV} \tau_{\chi_{1}^{0}}=10000 \mathrm{~mm}$ & $83.2 \pm 12$ \\
\hline$m_{\mathrm{H}}=150 \mathrm{GeV}, m_{\chi_{1}^{0}}=120 \mathrm{GeV}$ & $4159.7 \pm 462$ \\
$m_{\mathrm{H}}=200 \mathrm{GeV}, m_{\chi_{1}^{0}}=170 \mathrm{GeV}$ & $5963.3 \pm 660$ \\
$m_{\mathrm{H}}=300 \mathrm{GeV}, m_{\chi_{1}^{0}}=270 \mathrm{GeV}$ & $5152.6 \pm 571$ \\
$m_{\mathrm{H}}=400 \mathrm{GeV}, m_{\chi_{1}^{0}}=370 \mathrm{GeV}$ & $4056.8 \pm 450$ \\
\hline \hline
\end{tabular}

Upper limits are derived for two quantities: the product of the Higgs boson production cross section and $\mathcal{B}(\mathrm{H} \rightarrow$ invisible $+\gamma)$, and the ratio of this product over the SM Higgs production cross section 97,98$]$. This can be interpreted as the upper limit on $\mathcal{B}(\mathrm{H} \rightarrow$ invisible $+\gamma)$ assuming the SM production rate. To compute the upper limits the modified frequentist construction $\mathrm{CL}_{s}$ [99 101] is used. The number of events are modeled as a Poisson random variable, where the mean value is the sum of the contributions from signal and background processes. The expected and observed $95 \%$ confidence level (CL) limits for $m_{\mathrm{H}}=125 \mathrm{GeV}$ as a function of $m_{\chi_{1}^{0}}$ are shown in Figure 5.29. The 
expected and observed $95 \% \mathrm{CL}$ upper limits as a function of $m_{\mathrm{H}}$ with $m_{\chi_{1}}=m_{\mathrm{H}}-30 \mathrm{GeV}$ and for $m_{\mathrm{H}}=125 \mathrm{GeV}$ and $m_{\chi_{1}^{0}}=95 \mathrm{GeV}$ as a function of $\tau_{\chi_{1}^{0}}$ are shown in Figure 5.30 .
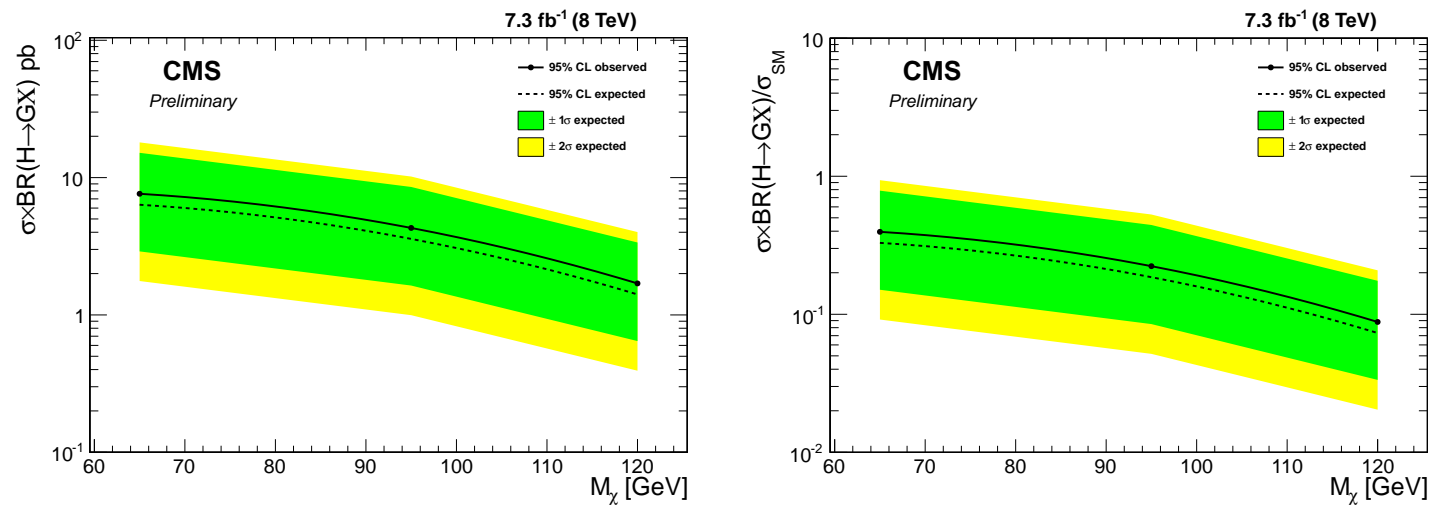

Figure 5.29: Expected and observed 95\% CL upper limits on (a) $\sigma \times B$ and (b) the ratio of this product over the SM Higgs production cross section as a function of different $M_{\widetilde{\chi}_{1}^{0}}$ values. The uncertainty on the expected limit at $1 \sigma$ and $2 \sigma$ levels are shown as green and yellow bands, respectively.
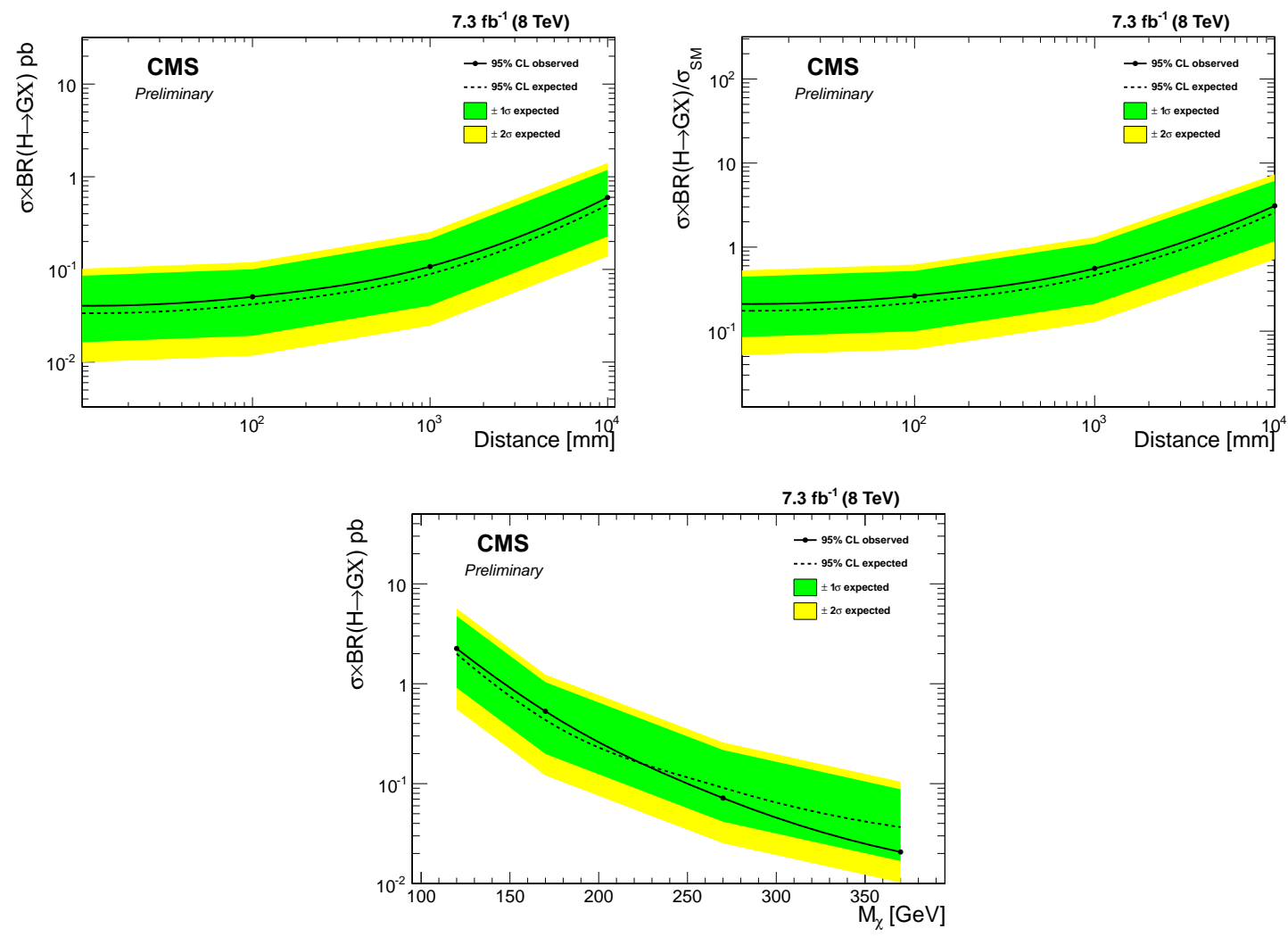

Figure 5.30: Expected and observed 95\% CL upper limits on mass with $\sigma \times \mathcal{B}$ and $\sigma \times \mathcal{B} / \sigma_{S M}$ as a function of $\tau_{\chi_{1}^{0}}$ for $m_{\mathrm{H}}=125 \mathrm{GeV}$ and $m_{\chi_{1}^{0}}=95 \mathrm{GeV}(\mathrm{up})$, where $\mathcal{B} \equiv \mathcal{B}\left(\mathrm{H} \rightarrow \chi_{1}{ }^{0} G\right) \times \mathcal{B}\left(\chi_{1}{ }^{0} \rightarrow\right.$ $G+\gamma$ ) and on $\sigma \times \mathcal{B}$ as a function of the Higgs boson mass with $m_{\chi_{1}}^{0}=m_{\mathrm{H}}-30 \mathrm{GeV}$ (bottom) 


\subsubsection{Extension to High-Energy Regime}

Mono-photon final state in higher energy regime was also studied extensively at the CMS experiment for three particular extensions of the SM: pair production of dark matter (DM) [102] particles, and two models with extra spatial dimensions.

DM particles $(\chi)$ at the LHC can be pair produced, where the photon will be radiated by one of the incoming quarks. The interaction is mediated by a virtual particle $M$ ("mediator"), with the mass much heavier than that of the DM particles. Various processes can be described with an effective theory description $103 \sqrt{106}$ where the contact interaction scale $\Lambda$, given by $\Lambda^{-2}=g_{\chi} g_{q} M_{M}^{-2}$, where $M_{M}$ is the mediator mass and $g_{\chi}$ and $g_{q}$ are its couplings to $\chi$ and quarks, respectively. This way, a comparison between direct searches relying upon $\chi$-nucleon scattering and the DM pair production can be made.

The ADD model [107] of large extra dimensions is postulated to have $n$ extra compactified spatial dimensions with a characteristic scale $R$. The true Planck scale, $M_{D}$ is given by $M_{\mathrm{Pl}}^{2} \approx M_{D}^{n+2} R^{n}$. This model predicts a sizable cross section for the process $q \bar{q} \rightarrow \gamma G$, where $G$ is a graviton which escapes detection, resulting in events with a single $\gamma$ and $\not_{T}$.

In the ADD approach, if one alternatively assumes that the brane may fluctuate in the extra dimensions, then the brane tension scale $f$ is expected to be much lower than other relevant scales such as $M_{D}$. The particles associated with these fluctuations of the brane are scalar particles called branons $[108$ 111]. Branons are stable and massive scalar dark matter candidates $[112]$ and can be abundantly pair-produced in association with SM particles at the LHC.

In this single-photon search background predictions were computed in a similar fashion, however, high energy single photon and photon- $\not{ }_{T}$ triggers that were $\sim 96 \%$ efficient for the selected signal region of $E_{\mathrm{T}}^{\gamma}>145 \mathrm{GeV}$, and $\not_{T}>140 \mathrm{GeV}$ were used. The data collected with the high energy triggers corresponds to an integrated luminosity of $19.6 \mathrm{fb}^{-1}$. Similar to the low energy analysis, candidate photons were restricted to the barrel region of the detector, and medium working point identification criteria was required for the photon candidates. In order to minimize the contribution from non-collision backgrounds photon candidate cluster was required to have a time consistent (within $\pm 3 \mathrm{~ns}$ ) with particles arriving from the collision. Events with more than one jet or a constructed lepton (electron or muon) with $p_{\mathrm{T}}>10 \mathrm{GeV}$ and $\Delta \mathrm{R}>0.5$ with respect to the photon were rejected. A topological requirement of $\Delta \phi\left(\overrightarrow{k_{T}}, \gamma\right)>2$ was required to minimize the contribution from $\gamma+$ jet background. To further suppress the mismeasured $\not_{T}$ backgrounds, $M H_{T}$ minimization

method was employed. Explicitly, $\operatorname{Prob}\left(\chi^{2}\right)$ to be less than $10^{-6}$ and the re-calculated $\not_{T}$ to be 
greater than $120 \mathrm{GeV}$ was required. These requirements removed $80 \%(35 \%)$ of $\gamma+$ jet (multijet) events while keeping $99.5 \%$ of signal events.

Table 5.9 shows the estimated number of events and uncertainty from each background for the entire dataset corresponding to $19.6 \mathrm{fb}^{-1}$ after the full event selection. Photon $E_{T}$ and $\not_{T}$ distributions for the selected events along with the estimated backgrounds and the spectrum expected from ADD for $M_{D}=2 \mathrm{TeV}$ and $n=3$ are shown in Fig. 5.31. It should be noted that limits were calculated using the $E_{\mathrm{T}}^{\gamma}$ distribution.

Table 5.9: Summary of estimated backgrounds and observed total number of candidates. Backgrounds listed as "Others" include the small contributions from, $Z \rightarrow l l \gamma, \gamma \gamma$ and $\gamma+$ jet. Uncertainties include both statistical and systematic contributions, and the total systematic uncertainty takes into account correlations in the individual estimates.

\begin{tabular}{|c|c|}
\hline Process & Estimate \\
\hline$Z(\rightarrow \nu \bar{\nu})+\gamma$ & $344.8 \pm 42.5$ \\
$W(\rightarrow \ell \nu)+\gamma$ & $102.5 \pm 20.6$ \\
$W \rightarrow e \nu$ & $59.5 \pm 5.5$ \\
jet $\rightarrow \gamma$ fakes & $45.4 \pm 13.9$ \\
Beam halo & $24.7 \pm 6.2$ \\
Others & $35.7 \pm 3.1$ \\
\hline Total background & $612.6 \pm 63.0$ \\
\hline \hline Data & 630 \\
\hline
\end{tabular}
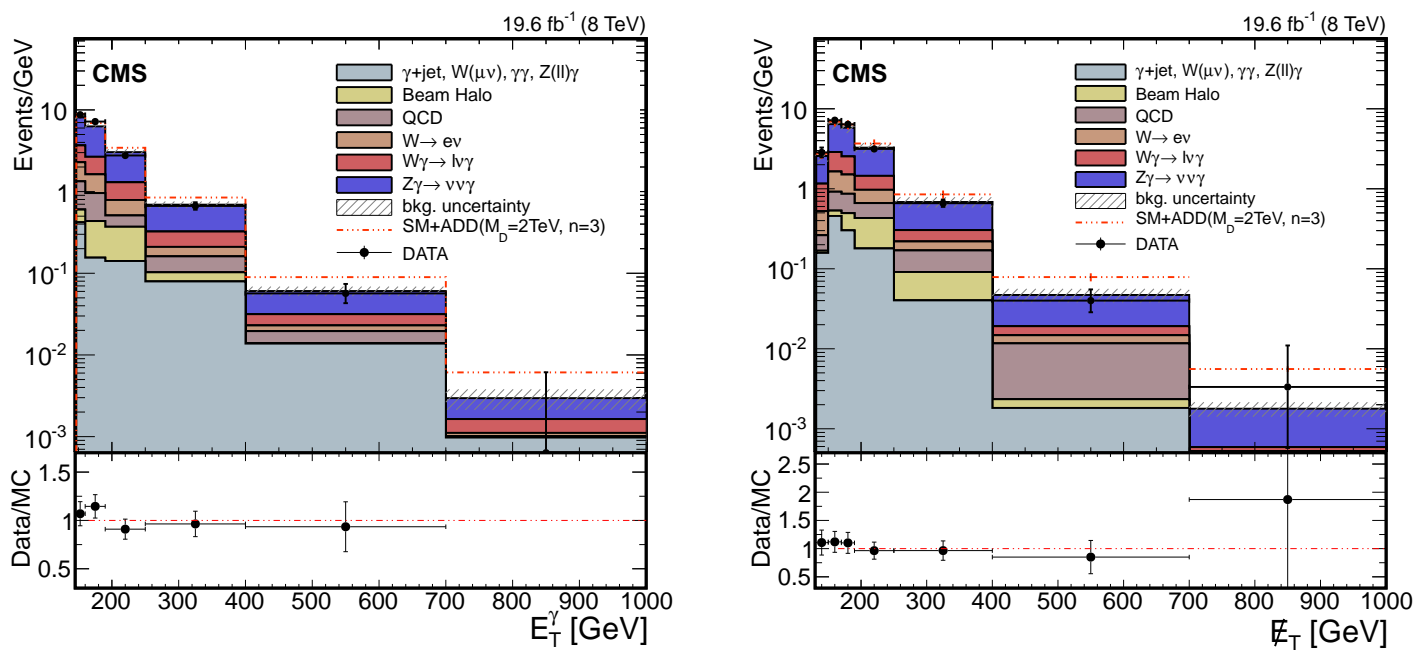

Figure 5.31: The photon $E_{T}$ and $\not_{T}$ distribution for the candidate sample, compared with estimated contributions from SM backgrounds and the prediction from ADD for $M_{D}=2 \mathrm{TeV}$ and $n=3$. Background uncertainty includes statistical and systematic uncertainties.

Model independent limits were computed using a $\mathrm{CL}_{\mathrm{s}}$ method [95, with uncertainties parametrized by log-normal distributions in the fit to data. The observed and expected upper limits on $\sigma \times A$ as a function of the cut on the photon $E_{T}$ for a generalized model of new physics 
are shown in Fig. 5.32 and in Table 5.10. The acceptance times efficiency, $A \times \epsilon$, were calculated by estimating $A \times \epsilon_{\mathrm{MC}}$ from the simulation after applying the scale factors to the simulation to account for the difference in efficiency between simulation and data.

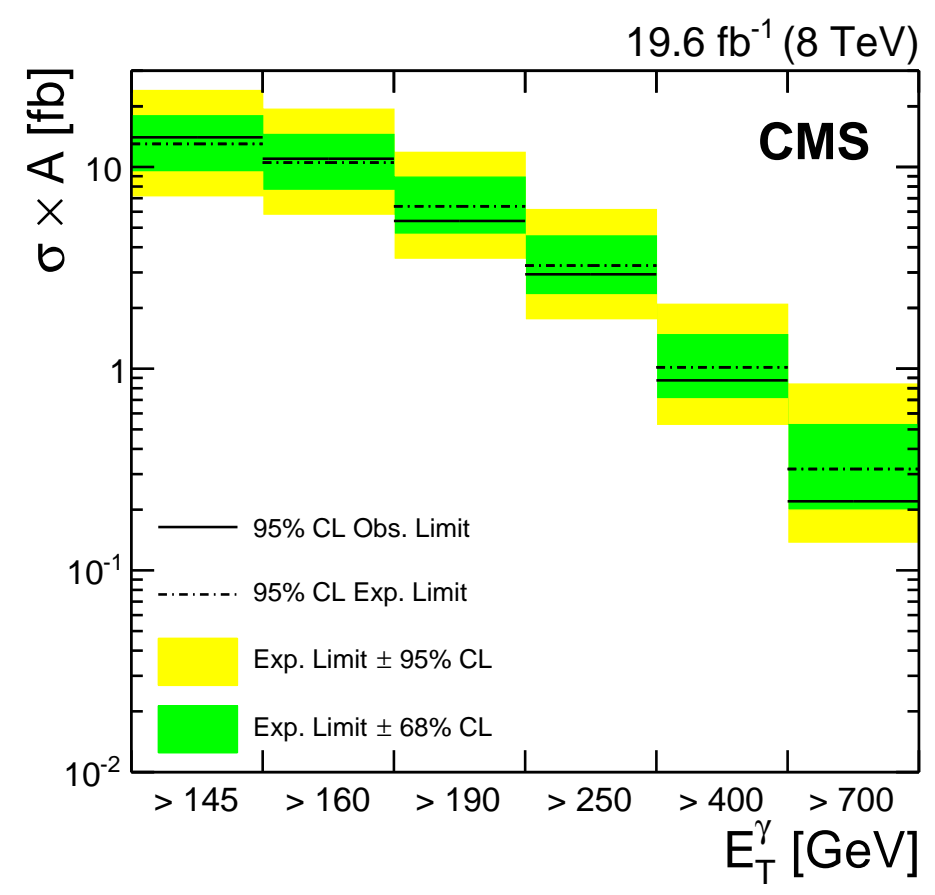

Figure 5.32: $95 \%$ upper limits on $\sigma \times A$ as a function of the cut on the photon $E_{T}$.

Table 5.10: Observed (Expected) 95\% CL and 90\% CL upper limits on $\sigma \times A$ as a function of the cut on the $E_{T}^{\gamma}$ for a generalized model of new physics. The requirement on $\not_{T}$ is fixed at $140 \mathrm{GeV}$.

\begin{tabular}{|c|c|c|}
\hline$E_{T}^{\gamma}[\mathrm{GeV}]$ & $\sigma \times A[\mathrm{fb}](95 \% \mathrm{CL})$ & $\sigma \times A[\mathrm{fb}](90 \% \mathrm{CL})$ \\
\hline 145 & $14.0(13.0)$ & $11.9(10.9)$ \\
160 & $11.0(10.5)$ & $9.29(8.84)$ \\
190 & $5.40(6.38)$ & $4.45(5.37)$ \\
250 & $2.94(3.24)$ & $2.43(2.70)$ \\
400 & $0.87(1.02)$ & $0.71(0.83)$ \\
700 & $0.22(0.32)$ & $0.16(0.25)$ \\
\hline
\end{tabular}

The lower limits on the interaction parameter $\Lambda$ were translated to upper limits on the darkmatter-nucleon scattering cross section using the formulae:

$$
\sigma_{\mathrm{SI}}=\frac{9}{\pi}\left(\frac{\mu}{\Lambda^{2}}\right)^{2}
$$

and 


$$
\sigma_{\mathrm{SD}}=\frac{0.33}{\pi}\left(\frac{\mu}{\Lambda^{2}}\right)^{2}
$$

for the spin independent and spin dependent interactions, respectively. $\mu$ is the reduced mass given in the form of:

$$
\mu \equiv\left(\frac{m_{\mathrm{DM}} m_{\mathrm{p}}}{m_{\mathrm{DM}}+m_{\mathrm{p}}}\right)
$$

The $m_{\mathrm{DM}}$ is the dark matter particle mass and $m_{\mathrm{p}}$ is the nucleon (specifically proton) mass respectively. The $90 \% \mathrm{CL}$ upper limits on the $\chi$-nucleon cross section as a function of $M_{\chi}$ for spin independent and spin dependent scattering are shown in Fig. 5.33, and Table 5.11 and Table 5.12 It should be noted that, the effective operator could be a mixture of vector and axial terms; for explicitness, the limiting cases of pure vector and pure axial vector operators have been chosen, corresponding to spin independent and spin dependent interactions, respectively.

Table 5.11: Theoretical DM production cross section where the generated photon transverse momentum is greater than $130 \mathrm{GeV}$ and the cut-off scale $\Lambda$ is $10 \mathrm{TeV}$, observed (expected) $90 \% \mathrm{CL}$ upper limits on the DM production cross section $\sigma, 90 \%$ CL lower limits on the cut-off scale $\Lambda$ and the $90 \%$ CL upper limits on the $\chi$-nucleon cross section for the vector operator as a function of the DM mass.

\begin{tabular}{|c|c|c|c|c|}
\hline Mass $[\mathrm{GeV}]$ & $\sigma_{\text {theor }}[\mathrm{fb}]$ & $\sigma[\mathrm{fb}]$ & $\Lambda[\mathrm{GeV}]$ & $\sigma_{\chi-\text { nucleon }}\left[\mathrm{cm}^{2}\right]$ \\
\hline 1 & $2.5 \mathrm{e}-4$ & $7.8(10.6)$ & $750(694)$ & $8.2 \mathrm{e}-40(1.1 \mathrm{e}-39)$ \\
10 & $2.5 \mathrm{e}-4$ & $8.0(10.5)$ & $745(696)$ & $2.6 \mathrm{e}-39(3.5-39)$ \\
100 & $2.4 \mathrm{e}-4$ & $8.0(11.2)$ & $742(684)$ & $3.2 \mathrm{e}-39(4.4 \mathrm{e}-39)$ \\
200 & $2.2 \mathrm{e}-4$ & $7.6(9.9)$ & $729(684)$ & $3.4 \mathrm{e}-39(4.4 \mathrm{e}-39)$ \\
300 & $1.8 \mathrm{e}-4$ & $6.9(9.4)$ & $714(660)$ & $3.7 \mathrm{e}-39(5.1 \mathrm{e}-39)$ \\
500 & $1.0 \mathrm{e}-4$ & $5.2(7.8)$ & $666(602)$ & $4.9 \mathrm{e}-39(7.4 \mathrm{e}-39)$ \\
1000 & $1.5 \mathrm{e}-5$ & $4.9(7.2)$ & $422(382)$ & $3.1 \mathrm{e}-38(4.6 \mathrm{e}-38)$ \\
\hline
\end{tabular}

Table 5.12: Theoretical DM production cross section where the generated photon transverse momentum is greater than $130 \mathrm{GeV}$ and the cut-off scale $\Lambda$ is $10 \mathrm{TeV}$, observed (expected) 90\% CL upper limits on the DM production cross section $\sigma, 90 \%$ CL lower limits on the cut-off scale $\Lambda$ and the $90 \%$ CL upper limits on the $\chi$-nucleon cross section for the axial vector operator as a function of the DM mass.

\begin{tabular}{|c|c|c|c|c|}
\hline Mass $[\mathrm{GeV}]$ & $\sigma_{\text {theor }}[\mathrm{fb}]$ & $\sigma[\mathrm{fb}]$ & $\Lambda[\mathrm{GeV}]$ & $\sigma_{\chi-\text { nucleon }}\left[\mathrm{cm}^{2}\right]$ \\
\hline 1 & $2.4 \mathrm{e}-4$ & $7.9(10.5)$ & $746(694)$ & $3.1 \mathrm{e}-41(4.1 \mathrm{e}-41)$ \\
10 & $2.5 \mathrm{e}-4$ & $7.9(11.0)$ & $748(688)$ & $9.6 \mathrm{e}-41(1.3 \mathrm{e}-40)$ \\
100 & $2.2 \mathrm{e}-4$ & $8.2(10.7)$ & $718(671)$ & $1.3 \mathrm{e}-40(1.7 \mathrm{e}-40)$ \\
200 & $1.6 \mathrm{e}-4$ & $6.7(9.5)$ & $702(643)$ & $1.5 \mathrm{e}-40(2.0 \mathrm{e}-40)$ \\
300 & $1.1 \mathrm{e}-4$ & $5.8(8.5)$ & $663(604)$ & $1.8 \mathrm{e}-40(2.6 \mathrm{e}-40)$ \\
500 & $4.9 \mathrm{e}-5$ & $5.5(8.1)$ & $544(495)$ & $4.0 \mathrm{e}-40(5.9 \mathrm{e}-40)$ \\
1000 & $4.2 \mathrm{e}-6$ & $5.3(7.7)$ & $298(272)$ & $4.5 \mathrm{e}-39(6.5 \mathrm{e}-39)$ \\
\hline
\end{tabular}

Recent studies on the validity of the EFT framework at the energy scale probed by the LHC 103 , 

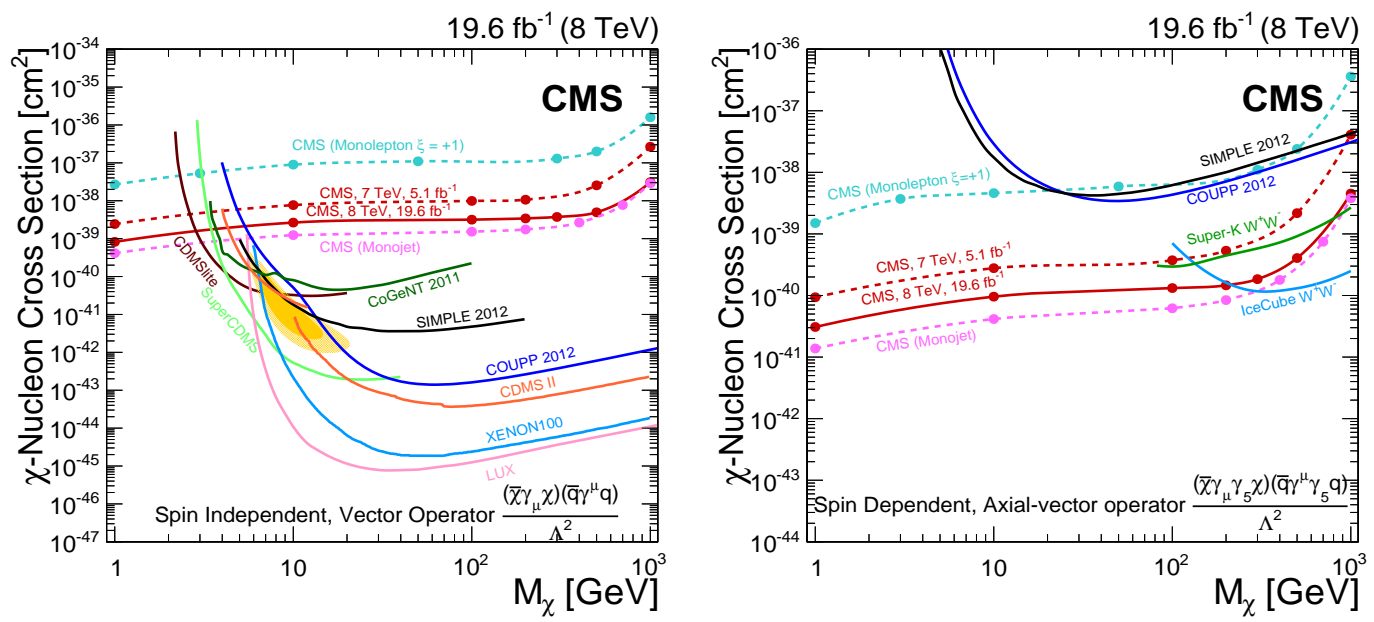

Figure 5.33: The 90\% CL upper limits on the $\chi$-nucleon cross section as a function of $M_{\chi}$ for spin independent (left) and spin dependent (right) scattering. Also shown are the limits from selected experiments with published 113 121] results.

104. 106 122 124 show that the for the EFT to be perturbative, $\sqrt{g_{\chi} g_{q}}$ is required to be less than $4 \pi$, a condition that is not valid over the entire phase space probed at LHC energies. Therefore, following [104, a simplified model predicting DM production via an s-channel vectorial mediator was considered. These limits are shown in Fig. 5.34. The mass of the mediator is varied for two fixed values of the mass of the $\mathrm{DM}$ particle: $50 \mathrm{GeV}$ and $500 \mathrm{GeV}$, and the width of the mediator is varied from $M / 8 \pi$ to $M / 3$. The contours for fixed values of $\sqrt{g_{\chi} g_{q}}$ are also shown for comparison. The results for a mediator with a mass of a few $\mathrm{TeV}$ are similar to those obtained from the EFT approach, while the limits are weaker at lower values of $M$.

A similar study has been performed with the low energy selection (as described in Section 5.6.2), with the goal of observing the resonant enhancement for the lower $M_{D M}$. Although the resonant enhancement is seen in the cross section due to the increased acceptance, no significant increase in sensitivity is observed due to the large background rate. However, it is worth noting that similar sensitivity to the high energy search is achieved with the lower energy selection using only one third of the data.

Upper limits at 95\% CL are also placed on the production cross section of the ADD and branon models, and translated into exclusions on the parameter space of the models. For the ADD model, the limits on $M_{D}$ for several values of $n$, the number of extra dimensions, are summarized in Table 5.13 These limits, along with existing ADD limits from the Tevatron [125, 126] and LEP [127 130, are shown in Fig. 5.36 as a function of $M_{D}$. 


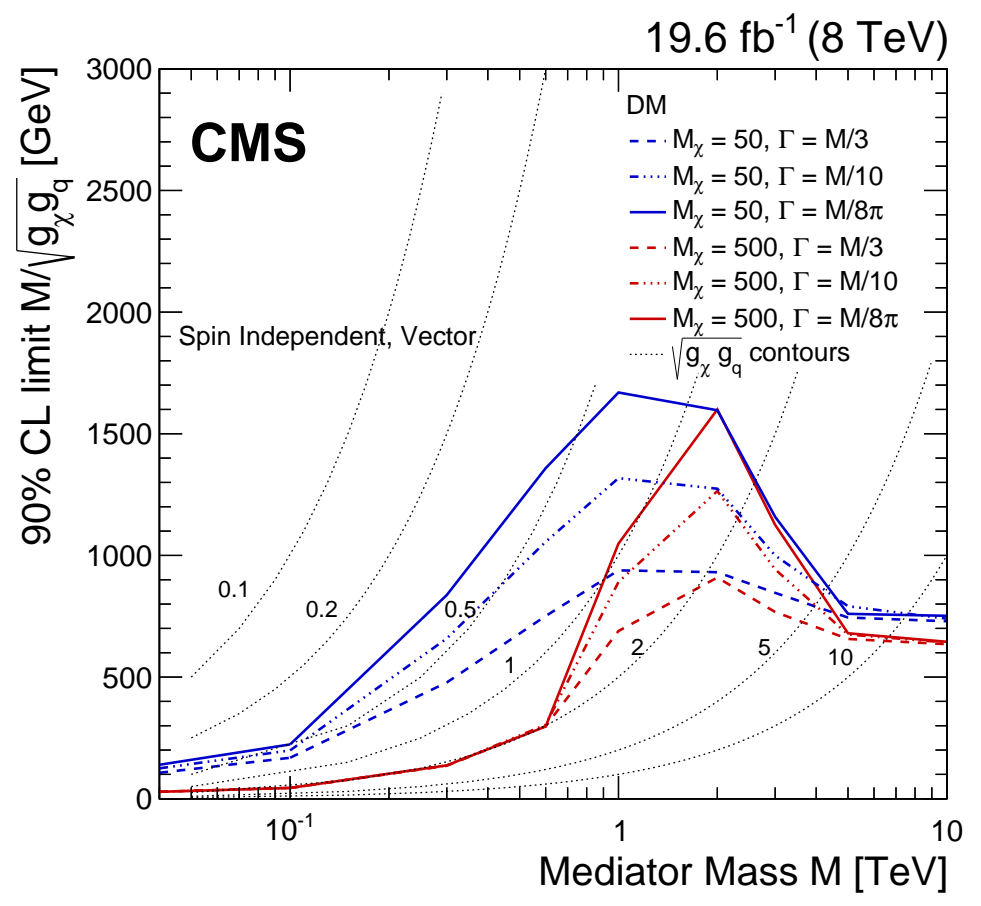

Figure 5.34: Observed limits on the DM mediator mass divided by coupling, $M / \sqrt{g_{\chi} g_{q}}$, as a function of the mediator mass $M$, assuming vector interactions, for DM particle masses of $50 \mathrm{GeV}$ and 500 $\mathrm{GeV}$. The width, $\Gamma$, of the mediator is varied between $M / 8 \pi$ and $M / 3$. The dashed lines show contours of constant coupling.
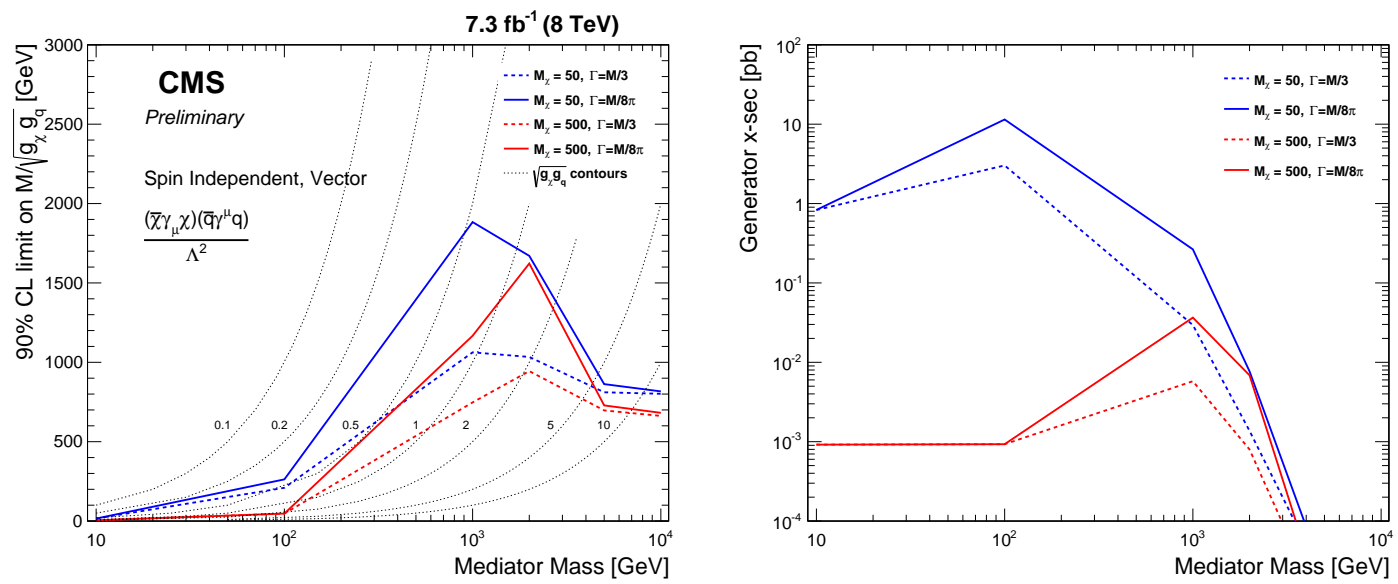

Figure 5.35: Observed limits on the DM mediator mass divided by coupling, $M / \sqrt{g_{\chi} g_{q}}$, as a function of the mediator mass $M$, assuming vector interactions, for DM particle masses of $50 \mathrm{GeV}$ and 500 $\mathrm{GeV}$ for the low energy search. The width, $\Gamma$, of the mediator is varied between $M / 8 \pi$ and $M / 3$. The dashed lines show contours of constant coupling.

Limits on $f$ for branons are summarized in Table 5.14 . For massless branons, the brane tension $f$ is found to be greater than $410 \mathrm{GeV}$ at $95 \% \mathrm{CL}$. These limits along with the existing limits from 
Table 5.13: Observed and expected 95\% CL upper limits on $M_{D}$ as a function of $n$

\begin{tabular}{|c|c|c|}
\hline$n$ & Obs. Limit $[\mathrm{TeV}]$ & Exp. Limit, $[\mathrm{TeV}]$ \\
\hline 3 & 2.30 & 1.99 \\
\hline 4 & 2.20 & 1.99 \\
\hline 5 & 2.04 & 1.99 \\
\hline 6 & 2.00 & 1.99 \\
\hline
\end{tabular}

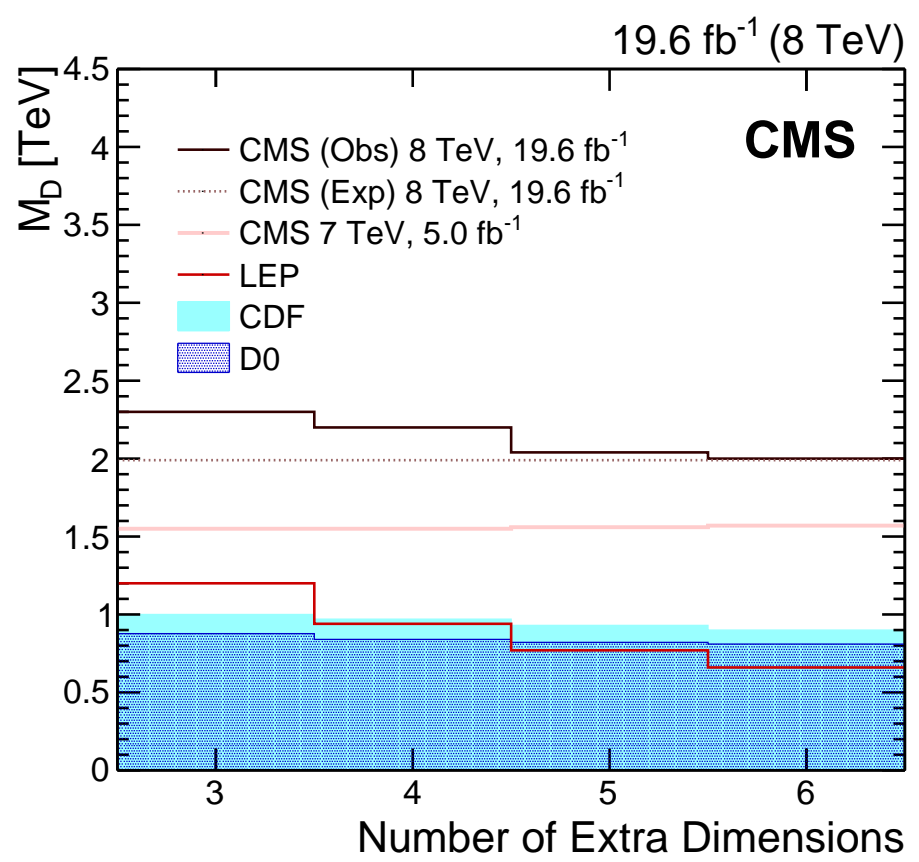

Figure 5.36: Limits on $M_{D}$ as a function of $n$, compared to results from similar searches at the Tevatron 125 126 and LEP 127.

LEP 131, are shown in Fig. 5.37. Branon masses $M_{B}<3.5 \mathrm{TeV}$ are excluded at 95\% CL for low brane tension $(20 \mathrm{GeV})$. These bounds are the most stringent published to date.

Table 5.14: Observed and expected 95\% CL upper limits on the branon brane tension $f$ as a function of the branon mass $M_{B}$.

\begin{tabular}{|c|cccccccccc|}
\hline$M_{B}[\mathrm{GeV}]$ & 100 & 500 & 1000 & 1500 & 2000 & 2500 & 2800 & 3000 & 3200 & 3500 \\
\hline Observed $f[\mathrm{GeV}]$ & 412 & 379 & 315 & 240 & 168 & 97 & 59 & 48 & 36 & 20 \\
\hline Expected $f[\mathrm{GeV}]$ & 400 & 373 & 308 & 238 & 167 & 97 & 59 & 48 & 36 & 20 \\
\hline
\end{tabular}



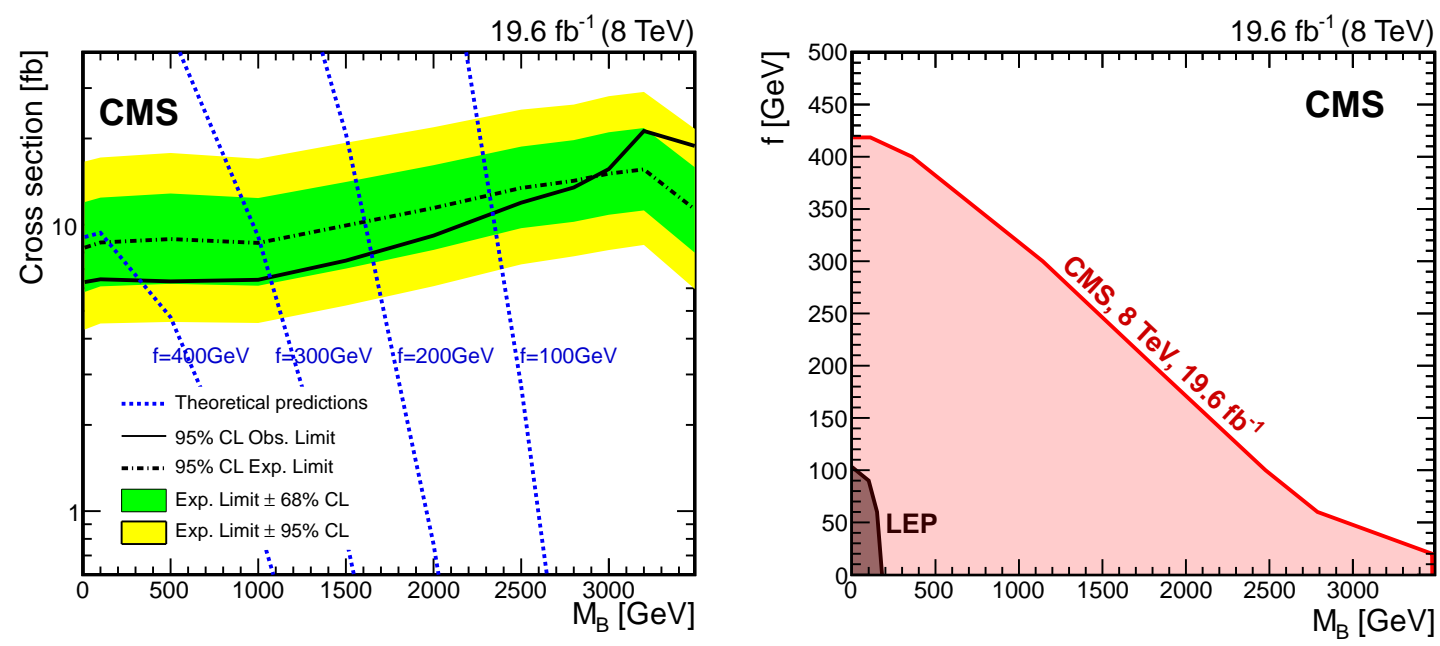

Figure 5.37: (a) The 95\% CL upper limits on the branon cross sections as a function of $M_{B}$ for $f=100,200,300$ and $400 \mathrm{GeV}$. (b) Limits on $f$ as a function of $M_{B}$, compared to results from similar searches at the LEP 131. 


\section{Chapter 6}

\section{Conclusion and Outlook}

A search for new physics in the $\gamma+\not{ }_{T}$ final state is performed using pp collision data corresponding to an integrated luminosity of $7.3 \mathrm{fb}^{-} 1$ collected at $\sqrt{s}=8 \mathrm{TeV}$ using low threshold triggers in a phase space region defined by $E_{\mathrm{T}}>45 \mathrm{GeV}$ and $\not_{T}>40 \mathrm{GeV}$. In the absence of any evidence of new physics, upper limits are placed on the production cross section of new physics in a modelindependent way for different $\not_{T}$ and $M_{T}$ thresholds. The data are also examined using optimized selections for maximum sensitivity to an exotic decay of the Higgs boson $h \rightarrow \widetilde{G} \widetilde{\chi}_{1}^{0}, \widetilde{\chi}_{1}^{0} \rightarrow \widetilde{G} \gamma$ predicted in a low-scale SUSY breaking scenario. Upper limits at 95\% CL are placed on the new physics production cross section times the branching ratio, as well as the ratio of this product to the SM Higgs boson production cross section. The results are found to be compatible with the Standard Model hypothesis. These results are the first limits on this model from collider searches.

Further more, proton-proton collision events containing high energy photon and missing transverse momentum have been investigated. No deviations from the standard model has been observed using the $\sqrt{s}=8 \mathrm{TeV}$ data set corresponding to $19.6 \mathrm{fb}^{-1}$ of integrated luminosity. In the absence of any evidence of new physics, bounds are placed on models predicting monophoton events; specifically, $95 \%$ confidence level upper limits for the cross section are set which vary from $14.0 \mathrm{fb}$ for $E_{\mathrm{T}}^{\gamma}>$ $145 \mathrm{GeV}$ to $0.22 \mathrm{fb}$ for $E_{\mathrm{T}}^{\gamma}>700 \mathrm{GeV}$. Further constraints are set on $\chi$ production and translated into upper limits on vector and axial-vector contributions to the $\chi$-nucleon scattering cross section. For $M_{\chi}=10 \mathrm{GeV}$, the $\chi$-nucleon cross section is constrained to be $2.6 \times 10^{-39} \mathrm{~cm}^{2}\left(9.6 \times 10^{-41}\right.$ $\mathrm{cm}^{2}$ ) for a spin-independent (spin-dependent) interaction at $90 \%$ confidence level. In addition the most stringent limits to date are obtained on the effective Planck scale in the ADD model with large spatial extra dimensions and on the brane tension scale in the branon model. 


\section{Bibliography}

[1] CMS Collaboration, "Observation of a new boson at a mass of $125 \mathrm{GeV}$ with the CMS experiment at the LHC," Physics Letters B $\mathbf{7 1 6}$ (2012) 30.

[2] ATLAS Collaboration, "Observation of a new particle in the search for the Standard Model Higgs boson with the ATLAS detector at the LHC," Physics Letters B 716 (2012) 1.

[3] P. W. Higgs, "Broken Symmetries and the Masses of Gauge Bosons," Physical Review Letters $13(1964) 508$.

[4] P. W. Higgs, "Spontaneous Symmetry Breakdown without Massless Bosons," Physical Review 145 (1966) 1156.

[5] F. Englert and R. Brout, "Broken Symmetry and the Mass of Gauge Vector Mesons," Physical Review Letters 13 (1964) 321.

[6] ATLAS Collaboration, "Updated coupling measurements of the Higgs boson with the ATLAS detector using up to $25 \mathrm{fb}^{-1}$ of proton-proton collision data," ATLAS-CONF-2014-009 (2014) .

[7] CMS Collaboration, "Combination of standard model Higgs boson searches and measurements of the properties of the new boson with a mass near $125 \mathrm{GeV}$," CMS-PAS-HIG-13-005 (2013) .

[8] M. Gell-Mann, "Symmetries of Baryons and Mesons,"Phys. Rev. 125 (Feb, 1962) 1067-1084. [link].

[9] S. Glashow, "Partial Symmetries of Weak Interactions," Nucl.Phys. 22 (1961) 579-588. [doi].

[10] S. Weinberg, "A Model of Leptons,"Phys. Rev. Lett. 19 (Nov, 1967) 1264-1266. [link]. 
[11] J. W. A.Salam, "Electromagnetic and weak interactions," Phys. Lett. 13 (1964) 168-171.

[12] J. Krumhansl and G. Trigg, "Publication of a New Discovery,"Phys. Rev. Lett. 33 (Dec, 1974) 1363-1363. [link].

[13] J. Augustin and et. al., "Discovery of a Narrow Resonance in $e^{+} e^{-}$Annihilation,"Phys. Rev. Lett. 33 (Dec, 1974) 1406-1408. [link].

[14] G. Arnison and et. al., "Experimental observation of isolated large transverse energy electrons with associated missing energy at s=540 GeV," Physics Letters B 122 (1983) no. 1, $103-116$. [link].

[15] M. Banner and et.al., "Observation of single isolated electrons of high transverse momentum in events with missing transverse energy at the CERN pp collider," Physics Letters B 122 (1983) no. 5, $476-485$. [link].

[16] J. B. et al. (Particle Data Group), "Review of PArticle Physics," Physical Review D 86 (2012) 010001.

[17] C. Quigg, Flavor Physics for the Millennium: TASI 2000, ch. The Electroweak Theory. World Scientific, 2000.

[18] M. G. et. al., "The standard model of Particle physics," Reviews of Modern Physics 71 (1999) S96.

[19] G. Altarelli, "The Standard Electroweak Theory and Beyond." arxiv:hep-ph/9811456, 1998.

[20] A. Pinch, "The Standard Model of Electroweak Interactions." arxiv:hep-ph/0705.4264, 2007.

[21] F. Halzen and A. Martin, Quarks and Leptons. Wiley, 1984.

[22] D. Griffiths, Introduction to Elementary Particles. John Wiley \& Sons, New York, USA, 1987.

[23] H. D. Politzer, "Reliable Perturbative Results for Strong Interactions?,"Phys. Rev. Lett. 30 (Jun, 1973) 1346-1349. [link]

[24] D. J. Gross and F. Wilczek, "Ultraviolet Behavior of Non-Abelian Gauge Theories," Phys. Rev. Lett. 30 (Jun, 1973) 1343-1346. [link]. 
[25] S. L. G. et. al., "Weak Interactions with Lepton-Hadron Symmetry," Physical Review D 2 (1970) 1285.

[26] P. W. Higgs, "Broken Symmetries and the Masses of Gauge Bosons," Physical Review Letters 13 (1964) 508.

[27] P. W. Higgs, "Spontaneous Symmetry Breakdown without Massless Bosons," Physical Review 145 (1966) 1156.

[28] F. Englert and R. Brout, "Broken Symmetry and the Mass of Gauge Vector Mesons," Physical Review Letters 13 (1964) 321.

[29] G. S. G. et. al., "Global Conservation Laws and Massless PArticles," Physical Review Letters $13(1965) 585$.

[30] J. Iliopoulos, "Introduction to the STANDARD MODEL of the Electro-Weak Interactions," 2012 CERN Summer School of Particle Physics, Angers : France (2012) . arXiv:1305.6779 [hep-ph].

[31] H. M. Georgi, S. L. Glashow, M. E. Machacek, and D. V. Nanopoulos, "Higgs Bosons from Two-Gluon Annihilation in Proton-Proton Collisions," Phys. Rev. Lett. 40 (Mar, 1978) 692-694. [link].

[32] L. Randall and R. Sundrum, "A Large mass hierarchy from a small extra dimension," Phys.Rev.Lett. 83 (1999) 3370-3373. arXiv:hep-ph/9905221 [hep-ph].

[33] B. Pontecorvo, "Neutrino Experiments and the Problem of Conservation of Leptonic Charge,"Soviet Journal of Experimental and Theoretical Physics 26 (May, 1968) 984.

[34] P. Fayet, "Mixing Between Gravitational and Weak Interactions Through the Massive Gravitino," Phys. Lett. B70 (1977) 461. [doi].

[35] H. Baer, M. Brhlik, C. H. Chen, and X. Tata, "Signals for the Minimal Gauge-Mediated Supersymmetry Breaking Model at the Fermilab Tevatron Collider," Phys. Rev. D55 (1997) 4463. [doi].

[36] H. Baer, P. G. Mercadante, X. Tata, and Y. L. Wang, "Reach of Tevatron Upgrades in Gauge-Mediated Supersymmetry Breaking Models," Phys. Rev. D60 (1999) 055001. [doi]. 
[37] S. Dimopoulos, S. Thomas, and J. D. Wells, "Sparticle Spectroscopy and Electroweak Symmetry Breaking with Gauge-Mediated Supersymmetry Breaking," Nucl. Phys. B488 (1997) 39. [doi].

[38] J. R. Ellis, J. L. Lopez, and D. V. Nanopoulos, "Analysis of LEP Constraints on Supersymmetric Models with a Light Gravitino," Phys. Lett. B394 (1997) 354. [doi].

[39] M. Dine, A. Nelson, Y. Nir, and Y. Shirman, "New Tools for Low Energy Dynamical Supersymmetry Breaking," Phys. Rev. D53 (1996) 2658. [doi].

[40] G. F. Giudice and R. Rattazzi, "Gauge-Mediated Supersymmetry Breaking," in Perspectives on Supersymmetry, p. 355. World Scientific, Singapore, 1998.

[41] L. Girardello and M. Grisaru, "Soft breaking of supersymmetry," Nuclear Physics B 194 (1982) no. $1,65-76$. [link].

[42] N. Polonsky, "Supersymmetry: Structure and phenomena. Extensions of the standard model," Lect.Notes Phys. M68 (2001) 1-169. arXiv:hep-ph/0108236 [hep-ph].

[43] G. R. Farrar and P. Fayet, "Phenomenology of the production, decay, and detection of new hadronic states associated with supersymmetry," Physics Letters B 76 (1978) no. 5, 575 579. [link].

[44] S. Deser and B. Zumino, "Broken Supersymmetry and Supergravity,"Phys. Rev. Lett. 38 (Jun, 1977) 1433-1436. [link]

[45] R. Casalbuoni, S. de Curtis, D. Dominici, F. Feruglio, and R. Gatto, "A gravitino-goldstino high-energy equivalence theorem," Phys. Lett. B 215 (1988) 313. [link].

[46] L. Evans and P. Bryant, "LHC Machine," JINST 3 (2008) S08001. [link].

[47] M. B. et. al., LHC Design Report - The LHC Injector Chain, vol. 3. CERN, 2004.

[48] "The CERN Accelerator Complex." http://te-epc-lpc.web.cern.ch/te-epc-lpc/ machines/pagesources/Cern-Accelerator-Complex.jpg. Accessed: 2013-12-27.

[49] ATLAS Collaboration, "ATLAS: technical proposal for a general-purpose pp experiment at the Large Hadron Collider at CERN," CERN-LHCC-94-43 .[1]ink]

[50] CMS Collaboration, "CMS technical proposal," CERN-LHCC-94-43 . [link]. 
[51] LHCb Collaboration, "LHCb technical proposal," CERN-LHCC-98-004 ·[link].

[52] ALICE Collaboration, "ALICE: Technical proposal for a Large Ion collider Experiment at the CERN LHC," CERN-LHCC-95-71 . [link].

[53] "CMS Luminosity - Public Results." https://twiki.cern.ch/twiki/bin/view/CMSPublic/LumiPublicResults. Accessed: 2014-02-18.

[54] CMS Collaboration, "The CMS Experiment at the CERN LHC," Journal of Instrumentation 3 (2008) S08004.

[55] CMS Collaboration, CMS Physics: Technical Design Report Volume 1: Detector Performance and Software. CERN, 2006. [link].

[56] "CMS detector design." http://cms.web.cern.ch/news/cms-detector-design. Accessed: 2013-12-27.

[57] CMS Collaboration, "Particle-Flow Event Reconstruction in CMS and Performance for Jets, Taus and Emiss," CMS-PAS-PFT-09-001. 2009.

[58] CMS Collaboration, "Identification of b-quark jets with the CMS experiment," JINST 8 (2013) P04013. [doi]

[59] CMS Collaboration, "Description and performance of track and primary-vertex reconstruction with the CMS tracker," vol. CMS-PAS-TRK-11-001. 2014.

[60] CMS Collaboration, "Studies of Tracker Material," vol. CMS-PAS-TRK-10-003. 2010.

[61] CMS Collaboration, "HCAL Technical Design Report," CERN LHC 97-31, CMS TDR .

[62] CMS Collaboration, "CMS The TRIDAS Project, Technical Design Report Vol. 2: Data Acquisition and High-Level Trigger," CERN LHC 02-26, CMS TDR 6 (2002) .

[63] CMS Collaboration, "CMS The TRIDAS Project, Technical Design Report Vol. 1: The Trigger Systems," CERN LHC 2000-38, CMS TDR 6.1 (2000) .

[64] P. Klabbers et al., "Integration of the CMS Regional Calorimeter Trigger Hardware into the CMS Level-1 Trigger," in Topical Workshop on Electronics for Particle Physics (TWEPP 2007). 2007. 
[65] P. Klabbers et al., "Operation and Monitoring of the CMS Regional Calorimeter Trigger Hardware," in Topical Workshop on Electronics for Particle Physics (TWEPP 2008). 2008.

[66] P. Klabbers et al., "Performance of the CMS Regional Calorimeter Trigger," in Topical Workshop on Electronics for Particle Physics (TWEPP 2009). 2009.

[67] CMS Collaboration, "The CMS High-Level Trigger," Eur. Phys. J. C 46 (2006) 605-667. [doi].

[68] S. Beauceron, "The CMS High Level Trigger," 36th International Conference on High Energy Physics. Melbourne, Australia, 2012.

[69] CMS Collaboration Collaboration, S. Chatrchyan et al., "The CMS experiment at the CERN LHC," JINST 3 (2008) S08004. [doi].

[70] CMS Collaboration Collaboration, "Particle-Flow Event Reconstruction in CMS and Performance for Jets, Taus, and MET,".

[71] M. Oreglia, A study of the reactions $\psi^{\prime} \rightarrow \gamma \gamma \psi$. PhD thesis, Stanford University, 1980. [link]. SLAC Report SLAC-R-236.

[72] CMS Collaboration, CMS, "Measurement of the inclusive $\mathrm{W}$ and $\mathrm{Z}$ production cross sections in pp collisions at $\sqrt{s}=7 \mathrm{TeV}, " J H E P 10$ (2011) 132. arXiv:1107.4789.

[73] CMS Collaboration, "Performance of photon reconstruction and identification in proton-proton collisions at $\sqrt{s}=8 \mathrm{TeV}$," paper in preparation.

[74] CMS Collaboration, CMS Collaboration, "Performance of electron reconstruction and selection at the CMS detector at $\sqrt{s}=8 \mathrm{TeV}$," paper in preparation.

[75] M. Cacciari and G. P. Salam, "Pileup subtraction using jet areas," Phys. Lett. B 659 (2008) 119. arXiv:0707.1378 [hep-ph]

[76] CMS Collaboration, S. Chatrchyan et al., "Determination of jet energy calibration and transverse momentum resolution in CMS," JINST 6 (2011) P11002. arXiv:1107.4277 [physics.ins-det]

[77] CMS Collaboration, CMS Collaboration, "Particle-Flow Event Reconstruction in CMS and Performance for Jets, Taus, and $\not_{T}$, , CMS Physics Analysis Summary CMS-PAS-PFT-09-001, 2009. [link] 
[78] CMS Collaboration Collaboration, S. Chatrchyan et al., "Determination of Jet Energy Calibration and Transverse Momentum Resolution in CMS," JINST 6 (2011) P11002. arXiv:1107.4277 [physics.ins-det]

[79] C. Petersson, A. Romagnoni, and R. Torre, "Higgs Decay with Monophoton + MET Signature from Low Scale Supersymmetry Breaking," JHEP 10 (2012) 016. arXiv: 1203.4563 [hep-ph]

[80] J. Alwall, P. Demin, S. de Visscher, R. Frederix, M. Herquet, F. Maltoni, T. Plehn, D. L. Rainwater, and T. Stelzer, "MadGraph/MadEvent v4: the new web generation," JHEP 09 (2007) 028. [doi]

[81] T. Sjöstrand, S. Mrenna, and P. Skands, "PYTHIA 6.4 physics and manual," JHEP 05 (2006) 026. arXiv:hep-ph/0603175 [hep-ph]

[82] H.-L. Lai, J. Huston, Z. Zi, P. Nadolsky, J. Pumplin, D. Stump, and C.-P. Yuan, "Uncertainty induced by QCD coupling in the CTEQ global analysis of parton distributions," Phys. Rev. D 82 (2010) 054021. arXiv:1004.4624 [hep-ph].

[83] GEANT4 Collaboration, S. Agostinelli et al., "GEANT4: A Simulation toolkit," Nucl. Instrum. Meth. A506 (2003) 250. [doi].

[84] A. Apresyan, "Identification and mitigation of anomalous signals in CMS HCAL," CMS Conference Report CMS-CR-2012-238, 2012.

[85] W. Bialas, D.A. Petyt, "Mitigation of anomalous APD signals in the CMS ECAL," JINST 8 (2013) C03020. [doi].

[86] CMS Collaboration, "Pileup Jet Identification," CMS Physics Analysis Summary CMS-PAS-JME-13-005, 2013. [link].

[87] CMS Collaboration, "Missing transverse energy performance of the CMS detector," JINST 6 (2011) P09001. arXiv:1106.5048 [physics.ins-det].

[88] J. Alwall, M. Herquet, F. Maltoni, O. Mattelaer, and T. Stelzer, "MadGraph 5 : Going Beyond," JHEP 1106 (2011) 128. arXiv:1106.0522 [hep-ph].

[89] J. Campbell, R. Ellis, and C. Williams, MCFM v6.1: A Monte Carlo for FeMtobarn processes at Hadron Colliders, 2011. [link]. 
[90] M. Botje, J. Butterworth, A. Cooper-Sarkar, A. de Roeck, J. Feltesse, S. Forte, A. Glazov, J. Huston, R. McNulty, T. Sjöstrand, and R. Thorne, "The PDF4LHC Working Group Interim Recommendations." 2011.

[91] CMS Collaboration, "CMS Luminosity Based on Pixel Cluster Counting - Summer 2013 Update," CMS Physics Analysis Summary CMS-PAS-LUM-13-001, 2013.

[92] CMS Collaboration, "Performance of photon reconstruction and selection in the CMS detector at $\sqrt{s}=8 \mathrm{TeV}$," paper in preparation.

[93] R. D. Ball, V. Bertone, F. Cerutti, L. Del Debbio, S. Forte, et al., "Impact of Heavy Quark Masses on Parton Distributions and LHC Phenomenology," Nucl. Phys. B 849 (2011) 296-363. arXiv:1101.1300 [hep-ph]

[94] A. Martin, W. Stirling, R. Thorne, and G. Watt, "Parton distributions for the LHC," Eur. Phys. J. C 63 (2009) 189. arXiv:0901.0002 [hep-ph]

[95] A. L. Read, "Presentation of search results: The CL(s) technique," J. Phys. G 28 (2002) 2693. [doi].

[96] T. Junk, "Confidence level computation for combining searches with small statistics," Nucl. Instrum. Meth. A 434 (1999) 435-443. arXiv:hep-ex/9902006 [hep-ex].

[97] LHC Higgs Cross Section Working Group, S. Dittmaier, C. Mariotti, G. Passarino, R. Tanaka (Eds.), "Handbook of LHC Higgs Cross Sections: Inclusive Observables," CERN Report CERN-2011-002, 2011. arXiv:1101.0593 [hep-ph].

[98] LHC Higgs Cross Section Working Group, S. Dittmaier, C. Mariotti, G. Passarino, R. Tanaka (Eds.), "Handbook of LHC Higgs Cross Sections: Differential Distributions," CERN Report CERN-2012-002, 2012. arXiv:1201.3084 [hep-ph].

[99] A. L. Read, "Presentation of search results: the CLs technique," J. Phys. G: Nucl. Part. Phys. 28 (2002) 2693. [doi].

[100] T. Junk, "Confidence level computation for combining searches with small statistics," Nucl. Instrum. Meth. A434 (1999) 435. [doi].

[101] ATLAS and CMS Collaborations, LHC Higgs Combination Group, "Procedure for the LHC Higgs boson search combination in Summer 2011," ATL-PHYS-PUB/CMS NOTE 2011-11, 2011/005, 2011. [link]. 
[102] R. Gaitskell, "Direct Detection of Dark Matter," Annual Review of Nuclear and Particle Science 54 (2004) 315. [doi]

[103] Y. Bai, P. J. Fox, and R. Harnik, "The Tevatron at the Frontier of Dark Matter Direct Detection," JHEP 12 (2010) 048. arXiv:1005.3797v2.

[104] P. J. Fox, R. Harnik, J. Kopp, and Y. Tsai, "Missing Energy Signatures of Dark Matter at the LHC," Phys.Rev. D85 (2012) 056011. arXiv:1109.4398 [hep-ph].

[105] J. Goodman, M. Ibe, A. Rajaraman, W. Shepherd, T. M. Tait, et al., "Constraints on Light Majorana dark Matter from Colliders," Phys.Lett. B695 (2011) 185-188. arXiv:1005.1286 [hep-ph].

[106] J. Goodman, M. Ibe, A. Rajaraman, W. Shepherd, T. M. Tait, et al., "Constraints on Dark Matter from Colliders," Phys.Rev. D82 (2010) 116010. arXiv:1008.1783 [hep-ph].

[107] N. Arkani-Hamed, S. Dimopoulos, and G. Dvali, "The Hierarchy problem and new dimensions at a millimeter," Phys. Lett. B 429 (1998) 263. arXiv:hep-ph/9803315 [hep-ph].

[108] R. Sundrum, "Effective field theory for a three-brane universe," Phys.Rev. D59 (1999) 085009. arXiv:hep-ph/9805471 [hep-ph].

[109] A. Dobado and A. L. Maroto, "The Dynamics of the Goldstone bosons on the brane," Nucl.Phys. B592 (2001) 203-218. arXiv:hep-ph/0007100 [hep-ph].

[110] J. Cembranos, A. Dobado, and A. L. Maroto, "Brane skyrmions and wrapped states," Phys.Rev. D65 (2002) 026005. arXiv:hep-ph/0106322 [hep-ph].

[111] J. A. R. Cembranos, R. L. Delgado, and A. Dobado, "Brane-Worlds at the LHC: Branons and KK-gravitons," Phys.Rev. D88 (2013) 075021. arXiv:1306.4900 [hep-ph].

[112] J. A. R. Cembranos, A. Dobado, and A. L. Maroto, "Cosmological and astrophysical limits on brane fluctuations,"Phys. Rev. D 68 (Nov, 2003) 103505. [link]

[113] XENON100 Collaboration, E. Aprile et al., "Dark Matter Results from 100 Live Days of XENON100 Data," Phys. Rev. Lett. 107 (2011) 131302. arXiv:1104.2549v3.

[114] CDMS II Collaboration, "Dark Matter Search Results from the CDMS II Experiment," Science 327 (2010) 1619. [link]. 
[115] CoGeNT Collaboration, C. E. Aalseth et al., "Results from a Search for Light-Mass Dark Matter with a p-Type Point Contact Germanium Detector," Phys. Rev. Lett. 106 (2011) 131301. arXiv:1002.4703.

[116] E. Behnke et al., "Improved Limits on Spin-dependent WIMP-Proton Interactions from a Two Liter $\mathrm{CF}_{3}$ Bubble Chamber," Phys. Rev. Lett. 106 (2011) 021303. arXiv:1008.3518.

[117] IceCube Collaboration, R. Abbasi et al., "Multiyear search for dark matter annihilations in the Sun with the AMANDA II and IceCube detectors," Phys. Rev. D 85 (2012) 042002. 1112.1840

[118] T. Tanaka et al., "An Indirect Search for Weakly Interacting Massive Particles in the Sun Using 3109.6 Days of Upward-going Muons in Super-Kamiokande," Astrophys. J. 742 (2011) 78. 1108.3384 .

[119] LUX Collaboration Collaboration, D. Akerib et al., "First results from the LUX dark matter experiment at the Sanford Underground Research Facility," Phys.Rev.Lett. 112 (2014) 091303. arXiv:1310.8214 [astro-ph.C0].

[120] SuperCDMS Soudan Collaboration, R. Agnese et al., "CDMSlite: A Search for Low-Mass WIMPs using Voltage-Assisted Calorimetric Ionization Detection in the SuperCDMS Experiment," Phys.Rev.Lett. 112 (2014) 041302. arXiv:1309.3259 [physics.ins-det].

[121] SIMPLE Collaboration, M. Felizardo, T. Morlat, A. C. Fernandes, T. A. Girard, J. G. Marques, A. R. Ramos, M. Auguste, D. Boyer, A. Cavaillou, C. Sudre, J. Poupeney, R. F. Payne, H. S. Miley, and J. Puibasset, "First Results of the Phase II SIMPLE Dark Matter Search," Phys. Rev. Lett. 105 (2010) 211301. [doi], A more recent update can be found in arXiv:1106.3014.

[122] H. An, X. Ji, and L.-T. Wang, "Light dark matter and $Z^{\prime}$ dark force at colliders," JHEP 07 (20 12) 182. arXiv:1202.2894 [hep-ph].

[123] A. Friedland, M. L. Graesser, I. M. Shoemaker, and L. Vecchi, "Probing nonstandard standard model backgrounds with LHC monojets," Phys. Lett. B 714 (2012) 267. arXiv:1111.5331 [hep-ph].

[124] O. Buchmueller, M. J. Dolan, and C. McCabe, "Beyond effective field theory for dark matter searches at the LHC," JHEP 01 (2014) 025. arXiv:1308.6799 [hep-ph]. 
[125] Do Collaboration, V. M. Abazov et al., "Search for Large Extra Dimensions via Single Photon plus Missing Energy Final States at $\sqrt{s}=1.96 \mathrm{TeV}, "$ Phys. Rev. Lett. 101 (2008) 011601. arXiv:0803.2137 [hep-ex].

[126] CDF Collaboration, T. Aaltonen et al., "Search for large extra dimensions in final states containing one photon or jet and large missing transverse energy produced in $\bar{p} \overline{\mathrm{p}}$ collisions at $\sqrt{s}=1.96$ TeV," Phys. Rev. Lett. 101 (2008) 181602. arXiv:0807.3132 [hep-ex].

[127] DELPHI Collaboration, J. Abdallah et al., "Photon events with missing energy in $\mathrm{e}^{+} \mathrm{e}^{-}$ collisions at $\sqrt{s}=130 \mathrm{GeV}$ to $209 \mathrm{GeV}, "$ Eur. Phys. J. C 38 (2005) 395. arXiv:hep-ex/0406019

[128] L3 Collaboration, P. Achard et al., "Single- and multi-photon events with missing energy in $\mathrm{e}^{+} \mathrm{e}^{-}$collisions at LEP," Phys. Lett. B 587 (2004) 16. arXiv:hep-ex/0402002 [hep-ex].

[129] OPAL Collaboration, G. Abbiendi et al., "Photonic events with missing energy in $\mathrm{e}^{+} \mathrm{e}^{-}$ collisions at $\sqrt{s}=189 \mathrm{GeV}, "$ Eur. Phys. J. C 18 (2000) 253. arXiv:hep-ex/0005002 [hep-ex]

[130] ALEPH Collaboration, A. Heister et al., "Single photon and multiphoton production in $\mathrm{e}^{+}$ $\mathrm{e}^{-}$collisions at $\sqrt{s}$ up to $209 \mathrm{GeV}, "$ Eur. Phys. J. C 28 (2003) 1. [doi].

[131] L3 Collaboration, P. Achard et al., "Search for branons at LEP," Phys. Lett. B 597 (2004) 145. arXiv:hep-ex/0407017 [hep-ex]. 